

\section{Algebra \& Number Theory}

www.jant.org

\section{EDITORS}

\author{
MANAGING EDITOR \\ Bjorn Poonen \\ Massachusetts Institute of Technology \\ Cambridge, USA
}

\author{
EDITORIAL BOARD CHAIR \\ David Eisenbud \\ University of California \\ Berkeley, USA
}

\section{BOARD OF EDITORS}

Georgia Benkart

Dave Benson

Richard E. Borcherds

John H. Coates

J-L. Colliot-Thélène

Brian D. Conrad

Hélène Esnault

Hubert Flenner

Edward Frenkel

Andrew Granville

Joseph Gubeladze

Ehud Hrushovski

Craig Huneke

Mikhail Kapranov

Yujiro Kawamata

János Kollár

Hendrik W. Lenstra

Yuri Manin

Barry Mazur
University of Wisconsin, Madison, USA

University of Aberdeen, Scotland

University of California, Berkeley, USA

University of Cambridge, UK

CNRS, Université Paris-Sud, France

University of Michigan, USA

Universität Duisburg-Essen, Germany

Ruhr-Universität, Germany

University of California, Berkeley, USA

Université de Montréal, Canada

San Francisco State University, USA

Hebrew University, Israel

University of Kansas, USA

Yale University, USA

University of Tokyo, Japan

Princeton University, USA

Universiteit Leiden, The Netherlands

Northwestern University, USA

Harvard University, USA
Susan Montgomery

Shigefumi Mori

Andrei Okounkov

Raman Parimala

Victor Reiner

Karl Rubin

Peter Sarnak

Michael Singer

Ronald Solomon

Vasudevan Srinivas

J. Toby Stafford

Bernd Sturmfels

Richard Taylor

Ravi Vakil

Michel van den Bergh

Marie-France Vignéras

Kei-Ichi Watanabe

Andrei Zelevinsky

Efim Zelmanov
University of Southern California, USA

RIMS, Kyoto University, Japan

Princeton University, USA

Emory University, USA

University of Minnesota, USA

University of California, Irvine, USA

Princeton University, USA

North Carolina State University, USA

Ohio State University, USA

Tata Inst. of Fund. Research, India

University of Michigan, USA

University of California, Berkeley, USA

Harvard University, USA

Stanford University, USA

Hasselt University, Belgium

Université Paris VII, France

Nihon University, Japan

Northeastern University, USA

University of California, San Diego, USA

\section{PRODUCTION}

ant@mathscipub.org

Paulo Ney de Souza, Production Manager

Silvio Levy, Senior Production Editor

See inside back cover or www.jant.org for submission instructions.

Regular subscription rate for 2010: $\$ 200.00$ a year (\$140.00 electronic only).

Subscriptions, requests for back issues from the last three years and changes of subscribers address should be sent to Mathematical Sciences Publishers, Department of Mathematics, University of California, Berkeley, CA 94720-3840, USA.

Algebra \& Number Theory, ISSN 1937-0652, at Mathematical Sciences Publishers, Department of Mathematics, University of California, Berkeley, CA 94720-3840 is published continuously online. Periodical rate postage paid at Berkeley, CA 94704, and additional mailing offices.

\section{PUBLISHED BY \\ athematical sciences publishers \\ http://www.mathscipub.org}

A NON-PROFIT CORPORATION

Typeset in IATEX

Copyright $\odot 2010$ by Mathematical Sciences Publishers 


\title{
Canonical extensions of Néron models of Jacobians
}

\author{
Bryden Cais
}

\begin{abstract}
Let $A$ be the Néron model of an abelian variety $A_{K}$ over the fraction field $K$ of a discrete valuation ring $R$. By work of Mazur and Messing, there is a functorial way to prolong the universal extension of $A_{K}$ by a vector group to a smooth and separated group scheme over $R$, called the canonical extension of $A$. Here we study the canonical extension when $A_{K}=J_{K}$ is the Jacobian of a smooth, proper and geometrically connected curve $X_{K}$ over $K$. Assuming that $X_{K}$ admits a proper flat regular model $X$ over $R$ that has generically smooth closed fiber, our main result identifies the identity component of the canonical extension with a certain functor $\operatorname{Pic}_{X / R}^{\natural, 0}$ classifying line bundles on $X$ that have partial degree zero on all components of geometric fibers and are equipped with a regular connection. This result is a natural extension of a theorem of Raynaud, which identifies the identity component of the Néron model $J$ of $J_{K}$ with the functor $\mathrm{Pic}_{X / R}^{0}$. As an application of our result, we prove a comparison isomorphism between two canonical integral structures on the de Rham cohomology of $X_{K}$.
\end{abstract}

\section{Introduction}

Fix a discrete valuation ring $R$ with field of fractions $K$ and residue field $k$. Let $A_{K}$ be an abelian variety over $K$ and consider the universal extension $E\left(\widehat{A}_{K}\right)$ of the dual abelian variety $\widehat{A}_{K}$. This commutative algebraic $K$-group is an extension of $\widehat{A}_{K}$ by the vector group of invariant differentials on $A_{K}$

$$
0 \longrightarrow \omega_{A_{K}} \longrightarrow E\left(\widehat{A}_{K}\right) \longrightarrow \widehat{A}_{K} \longrightarrow 0
$$

and is universal among extensions of $\widehat{A}_{K}$ by a vector group: for any vector group $V$ over $K$, the natural homomorphism $\operatorname{Hom}\left(\omega_{A_{K}}, V\right) \rightarrow \operatorname{Ext}\left(\widehat{A}_{K}, V\right)$ arising by pushout from (1-1) is an isomorphism. The theory of the universal extension was

MSC2000: primary 14L15; secondary 14F30, 14F40, 11G20, 14K30, 14H30.

Keywords: canonical extensions, Néron models, Jacobians, relative Picard functor, group schemes,

Grothendieck's pairing, Grothendieck duality, integral structure, de Rham cohomology, abelian

variety, rigidified extensions.

This work was partially supported by the NSF grant DMS-0502170 and by a Rackham Predoctoral Fellowship. 
initiated by Rosenlicht [1958], who defined the notion and showed its existence for abelian varieties, and was subsequently taken up in [Tate 1958; Murre 1962; Grothendieck 1974; Messing 1972; 1973; Mazur and Messing 1974]. It is central to the definition of the Mazur-Tate $p$-adic height pairing [Mazur and Tate 1983; Coleman 1991], to Deligne's definition of the duality on the de Rham cohomology of $A_{K}$ [Deligne 1974, Section 10.2.7.3] (see also [Coleman 1991; 1998]), and to certain proofs of the comparison isomorphism between the $p$-adic étale and de Rham cohomologies of $A_{K}$ [Coleman 1984, Note added in proof; Wintenberger 1994].

As is well known, the Néron model $\widehat{A}$ of $\widehat{A}_{K}$ over $R$ provides a functorial extension of $\widehat{A}_{K}$ to a smooth commutative group scheme over $R$, and it is natural to ask if (1-1) can be functorially extended to a short exact sequence of smooth commutative $R$-groups as well. Such an extension is provided by the "canonical extension" $\mathscr{E}(\widehat{A})$ of $\widehat{A}$, introduced by Mazur and Messing [1974, I, Section 5]. When $\widehat{A}_{K}$ has good reduction, $\mathscr{E}(\widehat{A})$ coincides with the universal extension of (the abelian scheme) $\widehat{A}$ by a vector group, but in general, as an example of Breen and Raynaud shows (see Remarks 2.5), Néron models need not have universal extensions, and $\mathscr{E}(\widehat{A})$ seems to be the best substitute in such cases. Although they seem to be of fundamental importance, canonical extensions of Néron models have been little studied, and as far as we know, do not appear anywhere in the literature beyond their introduction in [Mazur and Messing 1974] and [Gross 1990, Section 15].

In this paper, we study the canonical extension $\mathscr{E}(\widehat{A})$ when $A_{K}=J_{K}$ is the Jacobian of a smooth proper and geometrically connected curve $X_{K}$ over $K$. In this situation, a famous theorem of Raynaud [Bosch et al. 1990, Section 9.7, Theorem 1] relates the identity component $\widehat{J}^{0}$ of $\widehat{J}$ to the relative Picard functor of any proper flat and normal model $X$ of $X_{K}$ that is "sufficiently nice".

Theorem 1.1 (Raynaud). Let $S=\operatorname{Spec} R$ and fix a proper flat and normal model $X$ of $X_{K}$ over $S$. Denote by $X_{1}, \ldots, X_{n}$ the (reduced) irreducible components of the closed fiber $X_{k}$. Suppose that the greatest common divisor of the geometric multiplicities of the $X_{i}$ in $X_{k}$ is equal to 1, and assume either that $k$ is perfect or that $X$ admits an étale quasisection. Then $\mathrm{Pic}_{X / S}^{0}$ is a smooth and separated $S$-group scheme and $J_{K}$ admits a Néron model $J$ of finite type. Moreover, the canonical morphism

$$
\operatorname{Pic}_{X / S}^{0} \longrightarrow \widehat{J}^{0}
$$

arising via the Néron mapping property from the canonical principal polarization of $J_{K}$ is an isomorphism if and only if $X$ has rational singularities. ${ }^{1}$

Our main result enhances Raynaud's theorem by providing a similar description of the identity component $\mathscr{E}(\widehat{J})^{0}$ of the canonical extension $\mathscr{E}(\widehat{J})$ of $\widehat{J}$.

${ }^{1}$ Recall that $X$ is said to have rational singularities if it admits a resolution of singularities $\rho: X^{\prime} \rightarrow X$ with $R^{1} \rho_{*} \mathscr{O}_{X^{\prime}}=0$. Trivially, any regular $X$ has rational singularities. 
Theorem 1.2. Let $X$ be a proper flat and normal model of $X_{K}$ over $S=\operatorname{Spec} R$. Suppose that the closed fiber of $X$ is geometrically reduced and that either $X$ is regular or that $k$ is perfect. Then there is a canonical homomorphism of short exact sequences of smooth group schemes over $S$

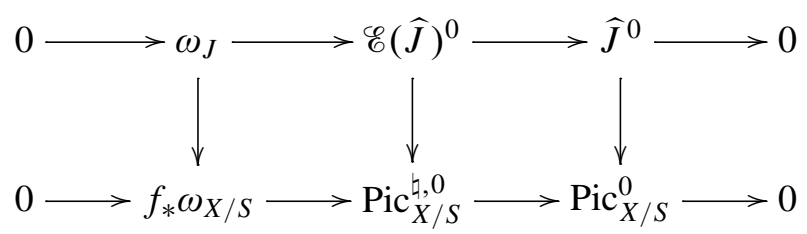

which is an isomorphism of exact sequences if and only if $X$ has rational singularities.

Here, $\omega_{X / S}$ is the relative dualizing sheaf of $X$ over $S$; it is a coherent sheaf of $\mathrm{O}_{X}$-modules that is flat over $S$ and coincides with the sheaf of relative differentials over the smooth locus of $f$ in $X$. We write $f_{*} \omega_{X / S}$ for the vector group attached to this locally free $O_{S}$-module, and $\operatorname{Pic}_{X / S}^{\natural, 0}$ is the fppf sheaf associated to the functor on $S$-schemes that assigns to each $S$-scheme $\varphi: T \rightarrow S$ the set of isomorphism classes of pairs $(\mathscr{L}, \nabla)$, where $\mathscr{L}$ is a line bundle on $X_{T}$ whose restriction to all components of each geometric fiber of $X_{T}$ has degree zero and $\nabla: \mathscr{L} \rightarrow \mathscr{L} \otimes \varphi^{*} \omega_{X / S}$ is a regular connection on $\mathscr{L}$ over $T$ (Definition 3.5). We will show in Theorem 3.9 that under the hypotheses of Raynaud's Theorem, $\mathrm{Pic}_{X / S}^{\natural, 0}$ is indeed a smooth and separated $S$-scheme, and that there is a short exact sequence of smooth groups over $S$ as in the lower row of (1-3).

We note that when $f: X \rightarrow S$ is smooth, our notion of regular connection coincides with the familiar notion of connection, and we recover from Theorem 1.2 the "well known" description of the universal extension of a Jacobian of a smooth and proper curve as the representing object of the functor classifying degree zero line bundles on the curve that are equipped with connection. ${ }^{2}$ Let us also point out that the hypotheses of Theorem 1.2 include not only all regular curves over $K$ with semistable reduction but many regular curves which are quite far $^{3}$ from having semistable reduction, such as the modular curves $X(N)$ and $X_{1}(N)$ over $K:=\mathbf{Q}_{p}\left(\zeta_{N}\right)$ for arbitrary $N$ (see [Katz and Mazur 1985, Theorems 13.7.6 and 13.11.4], which describe proper flat and regular models of $X(N)$ and $X_{1}(N)$, respectively, over $R=\mathbf{Z}_{p}\left[\zeta_{N}\right]$ that have geometrically reduced closed fibers).

It is well known that the exact sequence of Lie algebras arising from (1-1) is naturally isomorphic to the 3-term Hodge filtration exact sequence of the first de Rham

\footnotetext{
${ }^{2}$ Certainly this result appears in the literature — see for example [Coleman 1990, Section 2] — but we have been unable to find any proof of it. See, however [Mazur and Messing 1974, I, Section 4], which proves a result in a similar spirit.

${ }^{3}$ They achieve semistable reduction only after a large and wildly ramified extension of $K$.
} 
cohomology of $A_{K}$ (Proposition 5.1). Thus, the Lie algebra of the smooth $R$-group $\mathscr{E}(\widehat{A})$ provides a canonical $R$-lattice in the $K$-vector space $H_{\mathrm{dR}}^{1}\left(A_{K} / K\right)$ which is functorial in $K$-morphisms of $A_{K}$ (due to the Néron mapping property of $A$ and the functorial dependence of $\mathscr{E}(\widehat{A})$ on $A)$. When $A$ is an abelian scheme and the maximal ideal of $R$ has divided powers, Mazur and Messing proved [1974, II, Section 15] Grothendieck's conjecture [1974, V Section 5] that this $R$-lattice is naturally isomorphic to the Dieudonné module of the associated Barsotti-Tate group $A_{k}\left[p^{\infty}\right]$. Thus, Lie( $\left.\mathscr{E}(A)\right)$ provides a natural generalization of the Dieudonné module when $A$ is not an abelian scheme. In [Cais 2009], for a proper flat and normal $R$-curve $X$, we studied a canonical integral structure $H^{1}(X / R)$ on $H_{\mathrm{dR}}^{1}\left(X_{K} / K\right)$ (that is, an $R$-lattice that is functorial in $K$-morphisms of $X_{K}$ ) defined in terms of relative dualizing sheaves. It is natural to ask how $H^{1}(X / R)$ compares with the lattice $\operatorname{Lie}(\mathscr{E}(\widehat{J}))$ under the canonical identification $H_{\mathrm{dR}}^{1}\left(X_{K} / K\right) \simeq H_{\mathrm{dR}}^{1}\left(J_{K} / K\right)$. We will prove in Corollary 5.6 that these two lattices coincide when $X$ verifies the hypotheses of Theorem 1.2.

We briefly explain the main ideas underlying the proof of Theorem 1.2. Our first task is to reinterpret $\mathscr{E}(\widehat{J})^{0}$ as the representing object of the functor $\mathscr{E} x$ trig $_{S}\left(J, \mathbf{G}_{m}\right)$ on smooth $S$-schemes, à la Mazur and Messing [1974]. To do this, we must first show that the functor $\mathscr{E}^{\mathscr{E}} x t_{S}\left(J, \mathbf{G}_{m}\right)$ is represented by $\widehat{J}^{0}$ on smooth $S$-schemes, and by [Bosch 1997, Proposition 5.1] this holds if and only if Grothendieck's pairing on component groups is perfect. It follows from results of Bosch and Lorenzini [2002, Corollary 4.7] (see also Proposition 2.8) that the hypotheses of Theorem $1.2 \mathrm{imply}$ the perfectness of Grothendieck's pairing. However, we note that Grothendieck's pairing is not generally perfect (see Remark 2.9).

In Section 3, we construct the exact sequence of smooth $S$-group schemes occurring in the bottom row of (1-3). This is accomplished by Theorem 3.9, whose proof employs Čech-theoretical techniques to interpret the hypercohomology of the two-term complex $d \log : \mathbb{O}_{X}^{\times} \rightarrow \omega_{X / S}$ in terms of line bundles with regular connection, and makes essential use of the good cohomological properties of the relative dualizing sheaf and of Grothendieck duality. A key insight here is that the traditional notion of a connection on a line bundle on a scheme $X$ over a base $S$ is not well behaved when $X$ is not $S$-smooth and must be suitably modified as in Definition 3.5. With these preliminaries in place, we turn to the proof of Theorem 1.2 in Section 4. We must first construct a morphism of short exact sequences of smooth group schemes (1-3). Our strategy for doing this is as follows. Passing to an unramified extension of $K$ if need be, we suppose that $X_{K}$ has a rational point and use it to define an Albanese morphism $j_{K}: X_{K} \rightarrow J_{K}$. The Néron mapping property of $J$ allows us to extend $j_{K}$ to a morphism $j: X^{\text {sm }} \rightarrow J$ on the smooth locus of $f$ in $X$. By (functorially) pulling back rigidified extensions of $J$ by $\mathbf{G}_{m}$ along $j$, we get line bundles on $X^{\text {sm }}$ with connection. Via a careful analysis of the relative 
dualizing sheaf, we show in Lemma 4.1 that a line bundle with connection on $X^{\text {sm }}$ is equivalent to a line bundle with regular connection on $X$; this critically uses our hypothesis that the closed fiber of $X$ is geometrically reduced (equivalently, ${ }^{4}$ that $X^{\mathrm{sm}}$ is fiber-wise dense in $X$ ). From this, we deduce the desired map (1-3). To complete the proof of Theorem 1.2, we then "bootstrap" Raynaud's Theorem 1.1 using duality. Here, it is essential to know that the canonical evaluation duality between the Lie algebra of $J$ and the sheaf of invariant differentials on $J$ is compatible via $j$ with the (Grothendieck) duality of $f_{*} \omega_{X / S}$ and $R^{1} f_{*} O_{X}$. Such compatibility may be checked on generic fibers, where it is well known [Coleman 1998, Theorem 5.1].

We remark that when $k$ is perfect, both the short exact sequences of group schemes in the rows of (1-3) exist under the less restrictive hypotheses of Theorem 1.1; this follows immediately from Propositions 2.6 and 2.8 for the top row of (1-3), and from Theorem 3.9 for the bottom row. It is natural to ask if Theorem 1.2 holds in this generality as well. We do not know the answer to this question, as our construction of the map of short exact sequences of smooth groups in (1-3) seems to require the closed fiber of $X$ to be generically smooth. Indeed, our construction of (1-3) relies on extending an Albanese morphism $X_{K} \rightarrow J_{K}$ to some open subscheme $U$ of $X$ with the property that line bundles with connection on $U$ uniquely extend to line bundles with regular connection on $X$. On the one hand, this extension property seems to require $U$ to be fiber-wise dense in $X$ (see Lemma 4.1 and Remark 4.2), while on the other hand one only expects to be able to extend the morphism $X_{K} \rightarrow J_{K}$ to $U=X^{\mathrm{sm}}$. Thus, we are forced to require that $U=X^{\mathrm{sm}}$ be fiber-wise dense in $X$, that is, that $X_{k}$ be generically smooth (equivalently geometrically reduced). We note, however, that it is just our construction of the map (1-3) that requires $X$ to have generically smooth closed fiber; the proof that this map is an isomorphism of exact sequences of group schemes relies only on the weaker hypotheses of Raynaud's Theorem 1.1.

Conventions and notation. Fix a base scheme $S$. If $Y$ is any $S$-scheme and $S^{\prime} \rightarrow S$ is any morphism, we will often write $Y_{S^{\prime}}:=Y \times{ }_{S} S^{\prime}$ for the base change. When $S^{\prime}=\operatorname{Spec}(F)$ is the spectrum of a field, we will sometimes abuse notation and write $Y_{F}$ in place of $Y_{S^{\prime}}$. We will work with the fppf topology on the categories of $S$ schemes and of smooth $S$-schemes (see [SGA3-1 1970, Exposé IV, Section 6.3] or [Bosch et al. 1990, Section 8.1]); if $\mathscr{F}$ is any representable functor on one of these categories, we will also write $\mathscr{F}$ for the representing object. By an $S$-group scheme

\footnotetext{
${ }^{4}$ Indeed, if $X_{k}$ is geometrically reduced, it is clearly generically smooth. Conversely, as $X$ is normal by hypothesis, it must be $S_{2}$ by Serre's criterion for normality, whence $X_{k}$ is $S_{1}$. Since $X_{k}$ is also $R_{0}$ and " $R_{0}+S_{1}$ " is equivalent to being reduced, we conclude that $X_{k}$ is reduced and generically smooth, whence it must be geometrically reduced.
} 
$G$ we will always mean a finitely presented flat and separated commutative group scheme over $S$. As usual, we write $\mathbf{G}_{a}$ and $\mathbf{G}_{m}$ for the additive and multiplicative group schemes over $S$. A vector group on $S$ is any $S$-group that is Zariski-locally isomorphic to a product of copies of $\mathbf{G}_{a}$. Associated to any quasicoherent $\mathrm{O}_{S^{-}}$ module $\mathcal{M}$ is a sheaf for the fppf topology on $S$-schemes $\varphi: T \rightarrow S$ given by $\mathcal{M}(T):=\Gamma\left(T, \varphi^{*} \mathcal{M}\right)$. When $\mathcal{M}$ is locally free of finite rank, this fppf sheaf is represented by the vector group $\operatorname{Spec}\left(\operatorname{Sym}_{\mathscr{O}_{S}}\left(\mathcal{M}^{*}\right)\right)$, where $\mathcal{M}^{*}$ is the $O_{S}$-linear dual of $M$; we will frequently abuse notation and write $M$ for both the locally free ${ }_{S_{S}}$ module and the associated vector group on $S$. For any $S$-group $G$ with identity section $e: S \rightarrow G$, we put $\omega_{G}:=e^{*} \Omega_{G / S}^{1}$. As usual, for any $S$-scheme $T$ we put $T[\epsilon]:=T \times_{\mathbf{Z}} \operatorname{Spec}\left(\mathbf{Z}[\epsilon] / \epsilon^{2}\right)$, considered as a $T$-scheme via the first projection, and for any fppf sheaf $G$ we write $\mathscr{L i e}(G)$ for the fppf sheaf of $O_{S}$-modules defined (as in [Liu et al. 2004, Section 1]) by $\mathscr{L i e}(G)(T):=\operatorname{ker}(G(T[\epsilon]) \rightarrow G(T))$. When $G$ is a smooth group, this agrees with the traditional notion of relative Lie algebra (as a sheaf of $\mathcal{O}_{S}$-modules). We set $\operatorname{Lie}(G):=\mathscr{L i e}(G)(S)$.

\section{Canonical extensions of Néron models}

In this section, following [Mazur and Messing 1974], we recall the construction and basic properties of the canonical extension of a Néron model, and we explain how to interpret its identity component via rigidified extensions.

Let $S$ be any base scheme, and fix commutative $S$-group schemes $F$ and $G$. A rigidified extension of $F$ by $G$ over $S$ is a pair $(E, \sigma)$ consisting of an extension $E$ (of fppf sheaves of abelian groups over $S$ ) of $F$ by $G$

$$
0 \longrightarrow G \stackrel{l}{\longrightarrow} E \longrightarrow F \longrightarrow 0
$$

and a section $\sigma$ of $S$-pointed sheaves along the first infinitesimal neighborhood of the identity of $F$

$$
\operatorname{Inf}_{S}^{1}(F) \stackrel{\sigma}{\longrightarrow} E
$$

that projects to the canonical closed immersion $\operatorname{Inf}_{S}^{1}(F) \rightarrow F$. Two rigidified extensions $(E, \sigma)$ and $\left(E^{\prime}, \sigma^{\prime}\right)$ of $F$ by $G$ are called equivalent if there is a homomorphism (necessarily an isomorphism) $\varphi: E \rightarrow E^{\prime}$ that carries $\sigma$ to $\sigma^{\prime}$ and makes the diagram (2-3) commute:

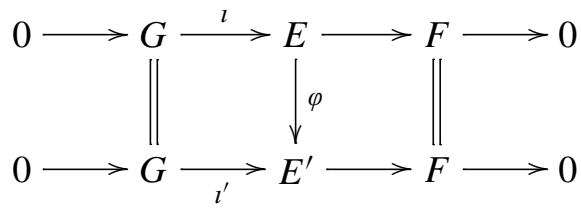


We denote by $\operatorname{Extrig}_{S}(F, G)$ the set of equivalence classes of rigidified extensions of $F$ by $G$ over $S$. This set is equipped with a natural group structure via Baer sum of rigidified extensions [Mazur and Messing 1974, I, Section 2.1]) which makes the functor on $S$-schemes $T \rightsquigarrow \operatorname{Extrig}_{T}\left(F_{T}, G_{T}\right)$ a group functor that is contravariant in the first variable via pullback (fibered product) and covariant in the second variable via pushout (fibered coproduct). We will write Extrig $(F, G)$ for the fppf sheaf of abelian groups associated to this functor.

We will exclusively be concerned with the special case that $G=\mathbf{G}_{m}$ is the multiplicative group over $S$. Note that (by fppf descent) any extension of $F$ by $\mathbf{G}_{m}$ is automatically representable as $\mathbf{G}_{m}$ is affine (compare the proof of [Oort 1966, III, Proposition 17.4]). In this context, there is an alternate and more concrete functorial description of the group $\operatorname{Extrig}_{S}\left(F, \mathbf{G}_{m}\right)$ that we will need for later use. Fix a choice of generator $\tau$ for the free rank-one $\mathbf{Z}$-module of invariant differentials $\omega_{\mathbf{G}_{m}}$ of $\mathbf{G}_{m}$ over $\mathbf{Z}$. Note that $\tau$ is canonically determined up to multiplication by \pm 1 . For any scheme $S$, we will denote the pullback of $\tau$ to a generator of $\omega_{\mathbf{G}_{m}}$ simply by $\tau$. Write $\mathbf{E}_{\tau}(F)(S)$ for the set of equivalence classes of pairs $(E, \eta)$ consisting of an extension $E$ of $F$ by $\mathbf{G}_{m}$ over $S$ and a global invariant differential $\eta \in \Gamma\left(S, \omega_{E}\right)$ which pulls back via the given morphism $\imath: \mathbf{G}_{m} \rightarrow E$ (realizing $E$ as an extension of $F$ by $\left.\mathbf{G}_{m}\right)$ to $\tau$ on $\mathbf{G}_{m}$. Two pairs $(E, \eta)$ and $\left(E^{\prime}, \eta^{\prime}\right)$ are declared to be equivalent if there is a morphism $\varphi: E \rightarrow E^{\prime}$ inducing a diagram as in (2-3) and having the property that $\varphi^{*} \eta^{\prime}=\eta$. We make $\mathbf{E}_{\tau}(F)(S)$ into an abelian group as follows. Let $(E, \eta)$ and $\left(E^{\prime}, \eta^{\prime}\right)$ be two pairs as above, and denote by $E^{\prime \prime}$ the Baer sum of $E$ and $E^{\prime}$. Writing pr, pr for the projections from $E \times{ }_{F} E^{\prime}$ to $E$ and $E^{\prime}$, and denoting by $q: E \times_{F} E^{\prime} \rightarrow E^{\prime \prime}$ the quotient map, we claim that there is a unique invariant differential $\eta^{\prime \prime}$ on $E^{\prime \prime}$ satisfying

$$
q^{*} \eta^{\prime \prime}=\operatorname{pr}^{*} \eta+\operatorname{pr}^{\prime *} \eta^{\prime}
$$

Indeed, by definition, $E^{\prime \prime}$ is the cokernel of the skew-diagonal $\left(l,-l^{\prime}\right): \mathbf{G}_{m} \rightarrow$ $E \times{ }_{F} E^{\prime}$ under which $\operatorname{pr}^{*} \eta+\operatorname{pr}^{\prime *} \eta^{\prime}$ pulls back to zero. Thus, via the short exact sequence

$$
0 \longrightarrow \omega_{E^{\prime \prime}} \longrightarrow \omega_{E \times{ }_{F} E^{\prime}} \longrightarrow \omega_{\mathbf{G}_{m}} \longrightarrow 0
$$

(which is left exact since $E \times_{F} E^{\prime} \rightarrow E^{\prime \prime}$ is smooth due to [SGA3-1 1970, Exposé $\mathrm{VI}_{\mathrm{B}}$, Proposition 9.2 vii]), we obtain a unique invariant differential $\eta^{\prime \prime}$ on $E^{\prime \prime}$ as claimed. One easily checks that under the map $\mathbf{G}_{m} \rightarrow E^{\prime \prime}$ induced by either one of the inclusions $(l, 0),\left(0, \imath^{\prime}\right): \mathbf{G}_{m} \rightrightarrows E \times_{F} E^{\prime}$ (whose composites with $q$ both coincide with the inclusion $\mathbf{G}_{m} \rightarrow E^{\prime \prime}$ realizing $E^{\prime \prime}$ as an extension of $F$ by $\mathbf{G}_{m}$ ) the differential $\eta^{\prime \prime}$ pulls back to $\tau$. We define the sum of the classes represented by $(E, \eta)$ and $\left(E^{\prime}, \eta^{\prime}\right)$ to be the class represented by $\left(E^{\prime \prime}, \eta^{\prime \prime}\right)$. It is straightforward to verify that this definition does not depend on the choice of representatives, and 
makes $\mathbf{E}_{\tau}(F)(S)$ into an abelian group. This construction is obviously contravariantly functorial in $S$ via pullback of extensions and of invariant differentials.

Lemma 2.1. For any choice of basis $\tau$ of $\omega_{\mathbf{G}_{m}}$, there is a functorial isomorphism of abelian groups

$$
\operatorname{Extrig}_{S}\left(F, \mathbf{G}_{m}\right) \stackrel{\simeq}{\longrightarrow} \mathbf{E}_{\tau}(F)(S) .
$$

Proof. Associated to the extension (2-1) with $G=\mathbf{G}_{m}$ is the short exact sequence of Lie algebras

$$
0 \longrightarrow \mathscr{L i e}\left(\mathbf{G}_{m}\right) \longrightarrow \mathscr{L i e}(E) \longrightarrow \mathscr{L i e}(F) \longrightarrow 0
$$

(note that the map $\mathscr{L i e}(E) \rightarrow \operatorname{Lie}(F)$ is surjective by [Liu et al. 2004, Proposition 1.1 (c)], as $E \rightarrow F$ is smooth). We claim that the data of a rigidification on (2-1) is equivalent to a choice of a splitting of (2-4). Indeed, any map $\sigma: \operatorname{Inf}_{S}^{1}(F) \rightarrow E$ necessarily factors through $\operatorname{Inf}_{S}^{1}(E)$, so using the natural isomorphism $\operatorname{Inf}_{S}^{1}(H) \simeq \operatorname{Spec}\left(O_{S}\left[\omega_{H}\right]\right)$ for any smooth group scheme $H$ over $S$ we obtain a bijection between rigidifications of (2-1) and sections $\omega_{E} \rightarrow \omega_{F}$ to the pullback map $\omega_{F} \rightarrow \omega_{E}$. By the usual duality of the $0_{S}$-modules $\mathscr{L} i e(H)$ and $\omega_{H}$ [SGA3-1 1970, Exposé 2, Section 4.11], this is equivalent to a section $s$ as claimed.

Using $\tau$ to identify the free rank one $\mathscr{O}_{S}$-module $\mathscr{L i e}\left(\mathbf{G}_{m}\right)$ with $\mathscr{O}_{S}$ and thinking of a splitting of (2-4) as a map $\mathscr{L i e}(E) \rightarrow \mathscr{L i e}\left(\mathbf{G}_{m}\right)$ restricting to the identity on $\mathscr{L i e}\left(\mathbf{G}_{m}\right)$, we see that any such splitting is by duality equivalent to a global section $\eta \in \Gamma\left(S, \omega_{E}\right)$ pulling back to $\tau$ in $\Gamma\left(S, \omega_{\mathbf{G}_{m}}\right)$. One checks that the equivalence $(E, \sigma) \leftrightarrow(E, \eta)$ induces an isomorphism of abelian groups $\operatorname{Extrig}_{S}\left(F, \mathbf{G}_{m}\right) \rightarrow$ $\mathscr{E}_{\tau}(F)(S)$ that is functorial in $S$, as claimed.

The following key result shows that the functor $\mathscr{E} x t r i g$ allows one to realize the universal extension of an abelian scheme.

Proposition 2.2 (Mazur-Messing). Let A be an abelian scheme over an arbitrary base scheme $S$ and denote by $\widehat{A}$ the dual abelian scheme. Then the fppf sheaf $\mathscr{E} x \operatorname{trig}_{S}\left(A, \mathbf{G}_{m}\right)$ is a smooth and separated $S$-group scheme. It sits in a natural short exact sequence of smooth $S$-group schemes

$$
0 \longrightarrow \omega_{A} \longrightarrow \mathscr{E} x \operatorname{trig}_{S}\left(A, \mathbf{G}_{m}\right) \longrightarrow \hat{A} \longrightarrow 0 \text {. }
$$

Moreover, (2-5) is the universal extension of $\widehat{A}$ by a vector group.

Proof. See [Mazur and Messing 1974], especially I, Section 2.6 and Proposition 2.6.7.

We now specialize to the case that $S=\operatorname{Spec} R$ is the spectrum of a discrete valuation ring $R$ with field of fractions $K$. Fix an abelian variety $A_{K}$ over $K$ and denote by $A$ the Néron model of $A_{K}$ over $S$ and by $A^{0}$ the relative identity 
component of $A$. Let $\widehat{A}$ be the Néron model of the dual abelian variety $\widehat{A}_{K}$. We have the following analogue of Proposition 2.2:

Proposition 2.3 [Mazur and Messing 1974, I, Corollary 5.2]. As a functor on smooth $S$-schemes, the fppf abelian sheaf $\mathscr{E x t r i g}\left(A_{S}^{0}, \mathbf{G}_{m}\right)$ is represented by a smooth and separated $S$-group scheme. Moreover, there is a natural short exact sequence of smooth groups over $S$

$$
0 \longrightarrow \omega_{A} \longrightarrow \mathscr{E} x \operatorname{trig}_{S}\left(A^{0}, \mathbf{G}_{m}\right) \longrightarrow \widehat{A} \longrightarrow 0
$$

Definition 2.4 (Mazur-Messing). The canonical extension of $\widehat{A}$ is the smooth and separated $S$-group scheme

$$
\mathscr{E}(\widehat{A}):=\mathscr{E} x \operatorname{trig}_{S}\left(A^{0}, \mathbf{G}_{m}\right) .
$$

Remarks 2.5. When $A$ is an abelian scheme, the canonical extension $\mathscr{E}(\widehat{A})$ coincides with the universal extension of $\widehat{A}$ by a vector group by Proposition 2.2. When $A$ is not an abelian scheme, an example of Breen and Raynaud [Mazur and Messing 1974, I, 5.6] shows that $A$ need not have a universal extension.

Note, however, that since the functor $\mathscr{E} x$ trig $_{S}\left(A^{0}, \mathbf{G}_{m}\right)$ commutes with fppf base change, the smooth group scheme $\mathscr{E} x t r i g_{S}\left(A^{0}, \mathbf{G}_{m}\right)$ representing it on the category of smooth group schemes over $S$ is of formation compatible with base change to a smooth $S$-scheme. In particular, the $K$-fiber of the canonical extension exact sequence (2-6) is the universal extension of $\widehat{A}_{K}$ by a vector group, thanks to Proposition 2.2.

In this paper, we work with $\mathscr{E} x \operatorname{trig}_{S}\left(A, \mathbf{G}_{m}\right)$ instead of $\mathscr{E} x t r i g_{S}\left(A^{0}, \mathbf{G}_{m}\right)$, as the former has better functorial properties due to the Néron mapping property of $A$ (which is not enjoyed by $A^{0}$ ). Following the method of [Mazur and Messing 1974, I, Corollary 5.2], we wish to show that $\mathscr{E} x \operatorname{trig}{ }_{S}\left(A, \mathbf{G}_{m}\right)$ is representable, at least as a functor on smooth test objects. This is somewhat more subtle than the corresponding problem for $\mathscr{E} x$ trig $_{S}\left(A^{0}, \mathbf{G}_{m}\right)$; in particular, denoting by $\Phi_{A}$ and $\Phi_{\widehat{A}}$ the component groups of $A$ and $\widehat{A}$, we will need to know that Grothendieck's pairing for $A_{K}$ (see [SGA7-1 1972, Exposés 7-9] or [Bosch 1997, Section 4])

$$
\Phi_{A} \times \Phi_{\widehat{A}} \longrightarrow \mathbf{Q} / \mathbf{Z}
$$

is right nondegenerate.

Proposition 2.6. Suppose that Grothendieck's pairing on component groups is right nondegenerate. Then the fppf abelian sheaf $\mathscr{E} x t r i g_{S}\left(A, \mathbf{G}_{m}\right)$ on the category of smooth $S$-schemes is represented by a smooth and separated $S$-group scheme. 
Moreover, there is a natural short exact sequence of smooth group schemes over $S$

$$
0 \longrightarrow \omega_{A} \longrightarrow \mathscr{E} x \operatorname{trig}_{S}\left(A, \mathbf{G}_{m}\right) \longrightarrow \widehat{A}^{0} \longrightarrow 0
$$

Proof. We follow the proof of [Mazur and Messing 1974, I, Corollary 5.2]. Let $T$ be any smooth $S$-scheme and consider the natural map of abelian groups

$$
\operatorname{Extrig}_{T}\left(A, \mathbf{G}_{m}\right) \longrightarrow \operatorname{Ext}_{T}\left(A, \mathbf{G}_{m}\right) .
$$

By Lemma 2.1, we see that when $T$ is affine any extension $E$ of $A_{T}$ by $\mathbf{G}_{m}$ admits a rigidification so (2-9) is surjective. By definition, the kernel of (2-9) consists of rigidifications on the trivial extension of $A_{T}$ by $\mathbf{G}_{m}$, up to isomorphism. To give a rigidification $\operatorname{Inf}_{T}^{1}\left(A_{T}\right) \rightarrow A_{T} \times_{T} \mathbf{G}_{m}$ of the trivial extension is obviously equivalent to giving a map of $T$-pointed $T$-schemes $\operatorname{Inf}_{T}^{1}\left(A_{T}\right) \rightarrow \mathbf{G}_{m}$, which in turn is equivalent to giving a global section of $\omega_{A_{T}}$ (see [Mazur and Messing 1974, I, 1.2] or the proof of Lemma 2.1). If two sections $\eta_{1}$ and $\eta_{2}$ of $\omega_{A_{T}}$ give isomorphic rigidified extensions of the trivial extension, then there is an automorphism of the trivial extension, necessarily induced by a group map $\varphi: A_{T} \rightarrow \mathbf{G}_{m}$, with the property that $\eta_{1}$ and $\eta_{2}$ differ by

$$
d \varphi \in \Gamma\left(T, \omega_{A_{T}}\right) \quad\left(\text { with } d: \operatorname{Hom}_{T}\left(A_{T}, \mathbf{G}_{m}\right) \rightarrow \operatorname{Hom}\left(\operatorname{Inf}_{T}^{1}\left(A_{T}\right), \mathbf{G}_{m}\right),\right.
$$

the natural map induced by the canonical closed immersion $\left.\operatorname{Inf}_{T}^{1}\left(A_{T}\right) \rightarrow A_{T}\right)$. Since $A$ is flat with proper generic fiber and $T$ is $S$-smooth, we have

$$
\operatorname{Hom}_{T}\left(A_{T}, \mathbf{G}_{m}\right)=0,
$$

so by passing to the associated fppf abelian sheaves, we thus obtain the short exact sequence of fppf sheaves

$$
0 \longrightarrow \omega_{A} \longrightarrow \mathscr{E} x \operatorname{trig}_{S}\left(A, \mathbf{G}_{m}\right) \longrightarrow \mathscr{E} x t_{S}\left(A, \mathbf{G}_{m}\right) \longrightarrow 0 \text {. }
$$

By [Bosch 1997, Proposition 5.1] (or [Milne 1986a, III, Proposition C.14]), the canonical duality of abelian varieties extends to a natural map $\widehat{A}^{0} \rightarrow \mathscr{E} x t_{S}\left(A, \mathbf{G}_{m}\right)$ which is an isomorphism of fppf abelian sheaves on the category of smooth $S$ schemes if and only if Grothendieck's pairing on component groups (2-7) is right nondegenerate. Thus, our hypotheses ensure that $\mathscr{E} x t_{S}\left(A, \mathbf{G}_{m}\right)$ is represented on the category of smooth $S$-schemes by the smooth and separated $S$-group scheme $\widehat{A}^{0}$. Since $\omega_{A}$ is a vector group, it is clearly smooth and affine over $S$. Thus, the proof of [Oort 1966, III, Proposition 17.4], which is easily adapted from the situation considered there (fpqc topology on all $S$-schemes) to our situation (fppf topology on smooth $S$-schemes) since $\omega_{A}$ and $\widehat{A}^{0}$ are smooth, shows via fppf descent that $\mathscr{E} x \operatorname{xrig}_{S}\left(A, \mathbf{G}_{m}\right)$ is represented (on smooth $S$-schemes) by a smooth and separated 
$S$-group scheme, and that there is a short exact sequence of smooth $S$-schemes (2-8).

Remark 2.7. We note that Mazur and Messing [1974, I, Corollary 5.2] prove that the canonical map

$$
\widehat{A} \longrightarrow \mathscr{E} x t_{S}\left(A^{0}, \mathbf{G}_{m}\right)
$$

is an isomorphism of fppf abelian sheaves on smooth test objects for any Néron model $A$ over any connected Dedekind scheme $S$ by showing that $\mathscr{E} x t_{S}\left(A^{0}, \mathbf{G}_{m}\right)$ satisfies the Néron mapping property. In our situation, this method fails to generalize as $\widehat{A}^{0}$ does not satisfy any good mapping property on smooth $S$-schemes which do not have connected closed fiber.

In our applications, we will wish to apply Proposition 2.6 when $A_{K}$ is the Jacobian of a smooth and proper curve over $K$. In this situation, it follows easily from the autoduality of $J_{K}$ and the functoriality of the morphism $\widehat{J}^{0} \rightarrow \mathscr{E} x t_{S}\left(J, \mathbf{G}_{m}\right)$ that Grothendieck's pairing is right nondegenerate if and only if it is left nondegenerate if and only if it is perfect. In order to apply Proposition 2.6, we will need the following criterion for the perfectness of Grothendieck's pairing.

Proposition 2.8. Let $X_{K}$ be a smooth and proper curve over $K$ with Jacobian $J_{K}$ over $K$. Fix a proper flat and normal model $X$ of $X_{K}$ over $R$, and denote by $X_{1}, \ldots, X_{n}$ the (reduced) irreducible components of the closed fiber $X_{k}$. Suppose that the greatest common divisor of the geometric multiplicities of the $X_{i}$ in $X_{k}$ is 1 , and assume one of the following hypotheses holds:

(1) The residue field $k$ of $R$ is perfect.

(2) $X$ is regular, each $X_{i}$ is geometrically reduced and $X$ admits an étale quasisection.

Then Grothendieck's pairing (2-7) for $J_{K}$ is perfect.

Proof. As our hypotheses are preserved by and our conclusion may be checked after étale base change, we may replace $R$ with a strict henselization of $R$ and may thus assume that $R$ is strictly henselian. In case (2), our hypotheses ensure that $X_{K}$ has a $K$-rational point and admits a proper flat and regular model $X$ over $R$ all of whose (reduced) irreducible components are geometrically reduced. These are exactly the hypotheses of [Bosch and Lorenzini 2002, Corollary 4.7], which then ensures that Grothendieck's pairing for $J_{K}$ is perfect.

In case (1), we first claim that our hypothesis on the gcd of the geometric multiplicities of the $X_{i}$ in $X_{k}$ imply the existence of a tamely ramified Galois extension $K^{\prime}$ of $K$ (necessarily with trivial residue field extension) such that $X_{K^{\prime}}$ has a $K^{\prime}$ rational point. Indeed, by resolution of singularities for excellent surfaces [Deligne and Mumford 1969, Section 2; Lipman 1978] and descent arguments from the 
completion of $R$ (see [Conrad et al. 2003, Theorem 2.2.2]) there exists a proper birational morphism of proper and flat $S$-models $\tilde{X} \rightarrow X$ of $X_{K}$ with $\tilde{X}$ regular. Due to [Liu 2002, Corollary 9.2.30], we may assume that the closed fiber $\widetilde{X}_{k}$ is a normal crossings divisor on $\widetilde{X}$. Observe that the proper and birational morphism $\widetilde{X} \rightarrow X$ is an isomorphism over any point $\xi \in X$ of codimension 1 ; this may be checked after the base change $\operatorname{Spec}\left(\mathcal{O}_{X, \xi}\right) \rightarrow X$, where it follows from the valuative criterion for properness applied to the discrete valuation ring $\mathcal{O}_{X, \xi}$ (recall that $X$ is normal). In particular, $\widetilde{X} \rightarrow X$ is an isomorphism over the generic points of $X_{k}$ and we deduce that our hypothesis on the gcd of the geometric multiplicities of the irreducible components of $X_{k}$ is inherited by $\widetilde{X}$. Thus, there exists an irreducible component $\Gamma_{0}$ of $\widetilde{X}_{k}$ whose multiplicity $e$ in $\widetilde{X}_{k}$ is not divisible by $\operatorname{char}(k)$. The proof of [Liu 2002, Theorem 10.4.6] (see also [Liu 2002, Corollary 10.4.7]) then shows that there is a Galois extension $K^{\prime}$ of $K$ with ramification index $e$ having the following property: letting $R^{\prime}$ denote the integral closure of $R$ in $K^{\prime}$, (which is again a discrete valuation ring, as $R$ is henselian) and writing $X^{\prime}$ for the normalization of $\widetilde{X} \times{ }_{S} \operatorname{Spec}\left(R^{\prime}\right)$, the closed fiber $X_{k}^{\prime}$ has an irreducible component $\Gamma_{0}^{\prime}$ over $\Gamma_{0}$ whose geometric multiplicity in $X_{k}^{\prime}$ is 1 ; that is, $\Gamma_{0}^{\prime}$ is generically smooth. As $R^{\prime}$ is strictly henselian, we conclude that there exists an $R^{\prime}$-point of $X^{\prime}$ and hence a $K^{\prime}$-point of $X_{K^{\prime}}^{\prime}=X_{K^{\prime}}$, as claimed.

Now since $k$ is perfect, $X_{K^{\prime}}$ admits a proper flat and regular model over $R^{\prime}$ with the property that every (reduced) irreducible component of the closed fiber is geometrically reduced (any proper flat and regular model will do). We may therefore apply [Bosch and Lorenzini 2002, Corollary 4.7] to $X_{K^{\prime}}$ to deduce that Grothendieck's pairing for $J_{K^{\prime}}$ is perfect. As $K^{\prime} / K$ is tamely ramified, it now follows from [Bertapelle and Bosch 2000] that Grothendieck's pairing for $J_{K}$ is perfect, as desired.

Remark 2.9. Assuming $k$ to be perfect, it follows from work of Pépin [2008] (using the results of Bosch and Lorenzini [2002]) that Grothendieck's pairing for $J_{K}$ is perfect whenever the index of $X_{K}$ is not divisible by the characteristic of $k$.

Already in the case of Jacobians, Grothendieck's pairing may fail to be perfect. Indeed, working over $R$ with imperfect residue fields, Bosch and Lorenzini give an explicit example of a Jacobian $J_{K}$ for which Grothendieck's pairing is not perfect [Bosch and Lorenzini 2002, Example 6.2]. The first examples of abelian varieties for which Grothendieck's pairing is not perfect were given by [Bertapelle and Bosch 2000].

For an arbitrary abelian variety $A_{K}$ over $K$, Grothendieck's pairing on component groups (2-7) is in addition known to be perfect under any of the following hypotheses:

(1) $R$ is of mixed characteristic $(0, p)$ and $k$ is perfect. 
(2) $k$ is finite.

(3) $k$ is perfect and $A_{K}$ has potentially multiplicative reduction.

(4) There exists a tamely ramified Galois extension $K^{\prime}$ of $K$ having trivial residue field extension such that Grothendieck's pairing for the base change $A_{K^{\prime}}$ is perfect.

For the proofs of these facts, see [Bégueri 1980] in the case of (1), [McCallum 1986] in case (2), [Bosch 1997] in case (3), and [Bertapelle and Bosch 2000] in the case of (4). See also [Milne 1986a, 3, Theorem 2.5] when $R$ has mixed characteristic and finite residue field.

We end this section by relating the group $\mathscr{E} x \operatorname{trig}{ }_{S}\left(A, \mathbf{G}_{m}\right)$ to the identity component of the canonical extension $\mathscr{E}(\widehat{A}):=\mathscr{E} x \operatorname{xrig}_{S}\left(A^{0}, \mathbf{G}_{m}\right)$ of $\widehat{A}$.

Lemma 2.10. Let $A_{K}$ be an abelian variety over $K$ and $A$ its Néron model over $R$. Suppose that Grothendieck's pairing (2-7) for $A_{K}$ is right nondegenerate, so $\mathscr{E x t r i g}{ }_{S}\left(A, \mathbf{G}_{m}\right)$ is a smooth $S$-group. The canonical map of short exact sequences of $S$-groups

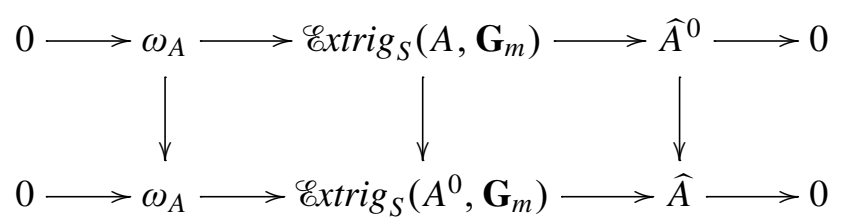

furnished from the functoriality of $\mathscr{E} x \operatorname{xrig}_{S}\left(\cdot, \mathbf{G}_{m}\right)$ by the inclusion $A^{0} \hookrightarrow A$ identifies $\mathscr{E} x \operatorname{xtrig}_{S}\left(A, \mathbf{G}_{m}\right)$ with the identity component of $\mathscr{E} x t r i g_{S}\left(A^{0}, \mathbf{G}_{m}\right)$.

Proof. We first observe that $\mathscr{E} x \operatorname{trig}_{S}\left(A, \mathbf{G}_{m}\right)$ has connected fibers. More generally, we claim that any extension of (not necessarily commutative) finite type connected group schemes over a field must be connected. Indeed, suppose that

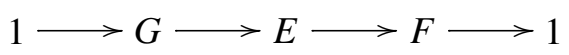

is such an extension. Since connectedness of any scheme with a rational point is preserved by ground field extension, the fibers of $E \rightarrow F$ are connected as they become isomorphic to $G$ after passing to an extension field and $G$ is connected. Thus, any separation $\{U, V\}$ of $E$ is a union of fibers of $E \rightarrow F$. Since the quotient map $E \rightarrow F$ is faithfully flat and of finite type, it is open, so $\{U, V\}$ is the pullback of a separation of $F$; by the connectedness of $F$ we conclude that $\{U, V\}$ is trivial and $E$ is connected.

To conclude, since $\mathscr{E} x \operatorname{trig}_{S}\left(A, \mathbf{G}_{m}\right)$ has connected fibers it suffices to show that any homomorphism from a commutative $S$-group $H$ with connected fibers to $\mathscr{E} x \operatorname{trig}_{S}\left(A^{0}, G_{m}\right)$ necessarily factors through $\mathscr{E} x t r i g_{S}\left(A, \mathbf{G}_{m}\right)$. By the functoriality of $\mathscr{E} x \operatorname{trig}_{S}\left(\cdot, \mathbf{G}_{m}\right)$, the top row of (2-10) is identified with the pullback of the bottom 
row along the inclusion $\widehat{A}^{0} \rightarrow \widehat{A}$; that is, we have a canonical isomorphism of smooth groups

$$
\mathscr{E} x \operatorname{trig}_{S}\left(A, \mathbf{G}_{m}\right)=\mathscr{E} x \operatorname{Etrig}_{S}\left(A^{0}, \mathbf{G}_{m}\right) \times_{\widehat{A}} \widehat{A}^{0}
$$

Thus, since the composition of $H \rightarrow \mathscr{E} x t r i g_{S}\left(A^{0}, \mathbf{G}_{m}\right)$ with the projection to $\widehat{A}$ necessarily factors through the inclusion of $\widehat{A}^{0}$ into $\widehat{A}$ as $H$ has connected fibers, we conclude that $H \rightarrow \mathscr{E} x$ trig $_{S}\left(A^{0}, \mathbf{G}_{m}\right)$ indeed factors through the fiber product $\mathscr{E} x \operatorname{trig}_{S}\left(A, \mathbf{G}_{m}\right)$, as desired.

\section{An enhancement of the relative Picard functor}

We continue to suppose that $S=\operatorname{Spec} R$ with $R$ a discrete valuation ring having field of fractions $K$. By a relative curve $X$ over $S$ we mean a flat finite type and separated $S$-scheme $f: X \rightarrow S$ of pure relative dimension 1 that is normal with smooth and geometrically connected generic fiber. In this section, we will introduce the functor $\mathrm{Pic}_{X / S}^{\natural, 0}$ and prove that it is representable whenever $\mathrm{Pic}_{X / S}^{0}$ is representable.

We begin by recalling some general facts about relative dualizing sheaves and Grothendieck duality that will be needed in what follows. Let $X$ and $Y$ be locally noetherian schemes and $f: X \rightarrow Y$ a Cohen-Macaulay morphism of pure relative dimension $n$. By [Conrad 2000, Theorem 3.5.1], the complex $f^{!} O_{Y}$ has a unique nonzero cohomology sheaf, which is in degree $-n$, and the relative dualizing sheaf of $X$ over $Y$ is

$$
\omega_{X / Y}:=H^{-n}\left(f^{!} \mathrm{O}_{Y}\right) .
$$

It is flat over $Y$ by [Conrad 2000, Theorem 3.5.1], and locally free if and only if the Cohen-Macaulay fibers of $f$ are Gorenstein [Hartshorne 1966, V, Proposition 9.3, Theorem 9.1]. Furthermore, the formation of $\omega_{X / Y}$ is compatible with étale localization on $X$ (see the discussion preceding [Conrad 2000, Corollary 4.4.5]) and with any base change $Y^{\prime} \rightarrow Y$ where $Y^{\prime}$ is locally noetherian [Conrad 2000, Theorem 3.6.1]. When $f$ is in addition proper, there is a natural $O_{Y}$-linear trace map

$$
\gamma_{f}: R^{n} f_{*} \omega_{X / Y} \rightarrow \mathrm{O}_{Y}
$$

which is compatible with any base change $Y^{\prime} \rightarrow Y$ with $Y^{\prime}$ locally noetherian [Conrad 2000, Corollary 3.6.6]. By Grothendieck-Serre duality [Conrad 2000, Theorem 4.3.1] the canonical map

$$
\mathbf{R} f_{*} \mathbf{R} \operatorname{lom}_{X}^{\bullet}\left(\mathscr{F} \bullet, \omega_{X / Y}[n]\right) \longrightarrow \mathbf{R} \mathscr{H o m}_{Y}^{\bullet}\left(\mathbf{R} f_{*} \mathscr{F}^{\bullet}, \mathcal{O}_{Y}\right)
$$

induced by (3-1) is a quasi-isomorphism for any complex $\mathscr{F}^{\bullet}$ in the derived category of sheaves of $\mathrm{O}_{X}$-modules whose cohomology is coherent and vanishes in sufficiently negative and positive degrees. 
For arbitrary base schemes $Y$ and Cohen-Macaulay morphisms $f: X \rightarrow Y$ of pure relative dimension, one defines $\omega_{X / Y}$ (and $\gamma_{f}$ when $f$ is proper) via direct limits and base change from the locally noetherian case (see [Conrad 2000, page 174]); this makes sense due to the aforementioned base-change compatibility in the locally noetherian context and yields a coherent sheaf of $0_{X}$-modules $\omega_{X / Y}$ and a trace map $\gamma_{f}$ when $f$ is proper that are compatible with arbitrary base change on $Y$.

Let us apply these considerations to the case of a relative curve $f: X \rightarrow S$. Since $X$ is normal and of pure relative dimension one, it is Cohen-Macaulay by Serre's criterion for normality. Theorem 23.3 in [Matsumura 1989]. Thus, the complex $f^{!} O_{S}$ is a coherent sheaf $\omega_{X / S}$ concentrated in degree -1 . By our discussion, $\omega_{X / S}$ is $S$-flat, and of formation compatible with étale localization on $X$ and arbitrary base change on $S$. When $f$ is $S$-smooth, the theory of the dualizing sheaf provides a canonical identification of the relative dualizing sheaf with the sheaf of relative differential 1-forms on $X$ over $S$. It is natural to ask how these two sheaves are related in general.

Proposition 3.1. There is a canonical $\mathrm{O}_{X}$-linear morphism

$$
c_{X / S}: \Omega_{X / S}^{1} \longrightarrow \omega_{X / S}
$$

whose restriction to any $S$-smooth open subset of $X$ is the canonical isomorphism. Proof. See [Cais 2009, Proposition 5.1].

In fact, we can realize $\omega_{X / S}$ as a subsheaf of differentials on $X$ which are regular on the generic fiber. Precisely, if $i: U \hookrightarrow X$ is any open subscheme of $X$ containing the generic fiber then the canonical map $\omega_{X / S} \rightarrow i_{*} i^{*} \omega_{X / S}$ is injective as it is an isomorphism over $X_{K}$ and $\omega_{X / S}$ is $S$-flat. Since the formation of $\omega_{X / S}$ is compatible with étale localization on $X$, we thus obtain a natural injective map

$$
\omega_{X / S} \longrightarrow i_{*} \omega_{U / S}
$$

In particular, taking $U=X_{K}$ we have $\omega_{U / S} \simeq \Omega_{X_{K} / K}^{1}$ by the general theory of the dualizing sheaf (or by Proposition 3.1), so $\omega_{X / S}$ is a subsheaf of $i_{*} \Omega_{X_{K} / K}^{1}$. When $U$ is large enough, the map (3-4) is also surjective.

Lemma 3.2. Suppose that the complement of $U$ in $X$ consists of finitely many closed points of codimension 2 (necessarily in the closed fiber). Then the canonical injective map (3-4) is an isomorphism.

Proof. We follow the proof given right after (5.2.7) in [Conrad 2000]. By standard arguments, it suffices to show that the local cohomology groups $H_{x}^{1}\left(X, \omega_{X / S}\right)$ 
vanish for all $x \in X-U$. Due to [SGA2 1968, Exposé III, Example 3.4], such vanishing is equivalent to

$$
\operatorname{depth}_{\mathscr{O}_{X, x}}\left(\omega_{X / S, x}\right) \geq 2 .
$$

If $x \in X-U$ is a regular point, this inequality is trivially verified, since $\omega_{X, x}$ is a free $\mathcal{O}_{X, x}$-module of rank 1 for such $x$ (regular local rings are Gorenstein) and $\mathcal{O}_{X, x}$ is two-dimensional and normal (whence it has depth 2 by Serre's criterion for normality).

In general, by [SGA2 1968, Exposé III, Corollary 2.5] it is enough to show that for each nonregular point $x$ of the closed fiber $X_{k}$ of $X$ we have

$$
\operatorname{depth}_{\mathscr{O}_{X_{k}, x}}\left(\omega_{X_{k} / k, x}\right) \geq 1 \text {. }
$$

If this is not the case, then the maximal ideal $\mathfrak{m}_{x}$ of $\mathfrak{O}_{X_{k}, x}$ consists entirely of zerodivisors for the finite $\mathrm{O}_{X_{k}, x}$-module $\omega_{X_{k} / k, x}$, so it must be an associated prime of $\omega_{X_{k} / k, x}$. We would then have $\mathfrak{m}_{x}=\operatorname{Ann}(s)$ for some nonzero $s \in \omega_{X_{k} / k, x}$ whence $\operatorname{Hom}_{X_{k}}\left(k(x), \omega_{X_{k} / k}\right) \neq 0$. However,

$$
\operatorname{Hom}_{X_{k}}\left(k(x), \omega_{X_{k} / k}\right)=H^{1}\left(X_{k}, k(x)\right)^{\vee}
$$

by Grothendieck duality for the $k$-scheme $X_{k}$ (see Corollary 5.1.3 and the bottom half of page 224 in [Conrad 2000]), and we know that the right side of (3-6) is zero (since $k(x)$ is a skyscraper sheaf supported at the point $x$ ), which is a contradiction. Thus, $\mathfrak{m}_{x}$ contains an $\omega_{X_{k} / k, x}$-regular element, so (3-5) holds, as desired.

When $f: X \rightarrow S$ is in addition proper, so we have a trace map (3-1), we may apply the machinery of Grothendieck duality. For our purposes, we need only the following.

Proposition 3.3. If $f: X \rightarrow S$ is a proper relative curve then the canonical map of flat $\mathrm{O}_{S}$-modules

$$
f_{*} \omega_{X / S} \longrightarrow\left(R^{1} f_{*} \mathrm{O}_{X}\right)^{\vee}
$$

induced by cup product and the trace map (3-1) is an isomorphism. Furthermore, there is a natural short exact sequence of $\mathrm{O}_{S}$-modules

$$
0 \longrightarrow \mathscr{E} x t_{S}^{1}\left(R^{1} f_{*} \mathrm{O}_{X}, \mathrm{O}_{S}\right) \longrightarrow R^{1} f_{*} \omega_{X / S} \longrightarrow\left(f_{*} \mathrm{O}_{X}\right)^{\vee} \longrightarrow 0 .
$$

In particular, if $f$ is cohomologically flat (in dimension 0 ) then $R^{1} f_{*} \omega_{X / S}$ is a locally free $\mathrm{O}_{S}$-module.

Proof. Since $\operatorname{Hom}_{X}\left(\mathrm{O}_{X}, \cdot\right)$ is naturally isomorphic to the identity functor, (3-2) with $\mathscr{F}^{\bullet}=\mathrm{O}_{X}$ (thought of as a complex in degree zero) yields a quasi-isomorphism

$$
\mathbf{R} f_{*} \omega_{X / S}[1] \simeq \mathbf{R} \mathscr{H o m}_{S}^{\bullet}\left(\mathbf{R} f_{*} \mathcal{O}_{X}, \mathcal{O}_{S}\right) .
$$


Applying $H^{-1}$ to (3-9) and using the spectral sequence

$$
E_{2}^{m, n}=\mathscr{E} x t_{S}^{m}\left(H^{-n}\left(\mathbf{R} f_{*} \mathcal{O}_{X}\right), \mathcal{O}_{S}\right) \Longrightarrow H^{m+n}\left(\mathbf{R} \operatorname{Hom}_{S}^{\bullet}\left(\mathbf{R} f_{*} \mathscr{O}_{X}, \mathscr{O}_{S}\right)\right)
$$

(whose only nonzero terms occur when $m=0,1$ and $n=0,-1$ ) to calculate the right side, we obtain a natural isomorphism $f_{*} \omega_{X / S} \simeq\left(R^{1} f_{*} O_{X}\right)^{\vee}$. To know that this map coincides with the map (3-7) induced by cup product and the trace map $\gamma_{f}$, one proceeds as in the proof of [Conrad 2000, Theorem 5.1.2]. Similarly, applying $H^{0}$ to (3-9) and using (3-10), we arrive at the short exact sequence (3-8). For the final statement of the proposition, recall that by definition $f$ is cohomologically flat in dimension 0 if $f_{*} \mathrm{O}_{X}$ commutes with arbitrary base change, which holds if and only if $R^{1} f_{*} O_{X}$ is locally free. Thus, when $f$ is cohomologically flat, the sheaf $\mathscr{E} x t_{S}^{1}\left(R^{1} f_{*} \mathrm{O}_{X}, O_{S}\right)$ vanishes and it follows easily from (3-8) that $R^{1} f_{*} \omega_{X / S}$ is locally free over $S$.

We record here a corollary showing that the relative dualizing sheaf is in general much better behaved than the sheaf of relative differential 1-forms:

Corollary 3.4. Let $f: X \rightarrow S$ be a proper relative curve, and assume that $f$ is cohomologically flat in dimension 0 . Then for all $i \geq 0$, the $\mathcal{O}_{S}$-module $R^{i} f_{*} \omega_{X / S}$ is locally free and commutes with arbitrary base change on $S$.

Proof. By standard arguments on base change, it is enough to show that $R^{i} f_{*} \omega_{X / S}$ is locally free for $i \geq 0$. This holds for $i \geq 2$ by the theorem on formal functions (as then $R^{i} f_{*} \omega_{X / S}=0$ ), and for $i=0$ since $\omega_{X / S}$ is $S$-flat. For $i=1$, it follows immediately from Proposition 3.3.

For a relative curve $f: X \rightarrow S$, we now wish to apply the preceding considerations to define a natural enhancement $\mathrm{Pic}_{X / S}^{\natural}$ of the relative Picard functor classifying invertible sheaves with the additional data of a "regular connection".

Let $T$ be any $S$-scheme. Since both the sheaf of relative differential 1-forms and the relative dualizing sheaf are compatible with base change, via pullback along $T \rightarrow S$ we obtain from (3-3) a natural morphism $\Omega_{X_{T} / T}^{1} \rightarrow \omega_{X_{T} / T}$, and hence an $\mathrm{O}_{T}$-linear derivation

$$
d_{T}: \mathrm{O}_{T} \longrightarrow \omega_{X_{T} / T} .
$$

Fix a line bundle $\mathscr{L}$ on $X_{T}$. Recall that a connection on $\mathscr{L}$ over $T$ is an $\bigcirc_{T}$-linear homomorphism $\nabla: \mathscr{L} \rightarrow \mathscr{L} \otimes_{\bigcirc_{T}} \Omega_{X_{T} / T}^{1}$ satisfying the usual Leibnitz rule. When $X$ is not $S$-smooth, this notion is not generally well behaved, and it is often desirable to allow connections to have certain types of poles along the singularities of $X$. For our purposes, the right notion of a connection is:

Definition 3.5. A regular connection on $\mathscr{L}$ over $T$ is an $\mathbb{O}_{T}$-linear homomorphism

$$
\nabla: \mathscr{L} \longrightarrow \mathscr{L} \otimes_{\mathbb{O}_{X_{T}}} \omega_{X_{T} / T}
$$


satisfying the Leibnitz rule: $\nabla(h \eta)=\eta \otimes d_{T}(h)+h \nabla \eta$ for any sections $h$ of $\mathcal{O}_{X_{T}}$ and $\eta$ of $\mathscr{L}$. A morphism of line bundles with regular connection over $T$ is an ${ }^{O_{X_{T}}}{ }^{-}$ linear morphism of the underlying line bundles that is compatible with the given connections.

Remark 3.6. Observe that any connection $\nabla: \mathscr{L} \rightarrow \mathscr{L} \otimes \Omega_{X_{T} / T}^{1}$ on $\mathscr{L}$ over $T$ gives rise to a regular connection on $\mathscr{L}$ over $T$ via composition with the map induced by $\Omega_{X_{T} / T}^{1} \rightarrow \omega_{X_{T} / T}$.

If $\mathscr{L}$ and $\mathscr{L}^{\prime}$ are two line bundles on $X_{T}$ equipped with regular connections $\nabla$ and $\nabla^{\prime}$ over $T$, the tensor product $\mathscr{L} \otimes_{\mathscr{O}_{X}} \mathscr{L}^{\prime}$ is naturally equipped with the tensor product regular connection $\nabla \otimes \nabla^{\prime}$ induced by decreeing

$$
\left(\nabla \otimes \nabla^{\prime}\right)\left(\eta \otimes \eta^{\prime}\right):=\eta \otimes \nabla^{\prime}\left(\eta^{\prime}\right)+\eta^{\prime} \otimes \nabla(\eta),
$$

for any sections $\eta$ of $\mathscr{L}$ and $\eta^{\prime}$ of $\mathscr{L}^{\prime}$. Observe that with respect to this operation, the pair $\left(\mathrm{O}_{X_{T}}, d_{T}\right)$ serves as an identity element. Thus, the set of isomorphism classes of line bundles on $X_{T}$ with a regular connection over $T$ has a natural abelian group structure which is obviously compatible with our definition of a morphism of line bundles with connection. Furthermore, if $T^{\prime} \rightarrow T$ is any morphism of $S$-schemes, then since the formation of $\omega_{X / S}$ is compatible with base change, any line bundle on $X_{T}$ with regular connection over $T$ pulls back to a line bundle on $X_{T^{\prime}}$ with regular connection over $T^{\prime}$.

Definition 3.7. Denote by $P_{X / S}^{\natural}$ the contravariant functor from the category of $S$ schemes to the category of abelian groups given on an $S$-scheme $T$ by

$$
P_{X / S}^{\natural}(T):=\left\{\begin{array}{l}
\text { Isomorphism classes of pairs }(\mathscr{L}, \nabla) \text { consisting of a line bundle } \\
\mathscr{L} \text { on } X_{T} \text { equipped with a regular connection } \nabla \text { over } T
\end{array}\right\},
$$

and write $\operatorname{Pic}_{X / S}^{\natural}$ for the fppf sheaf associated to $P_{X / S}^{\natural}$.

As is customary, we will denote by $P_{X / S}$ the contravariant functor on the category of $S$-schemes which associates to an $S$-scheme $T$ the set of isomorphism classes of line bundles on $X_{T}$, and by $\mathrm{Pic}_{X / S}$ the fppf sheaf on the category of $S$-schemes associated to $P_{X / S}$. For any $S$-scheme $T$, there is an obvious homomorphism of abelian groups $P_{X / S}^{\natural}(T) \rightarrow P_{X / S}(T)$ given by "forgetting the connection", and hence a map of fppf abelian sheaves

$$
\operatorname{Pic}_{X / S}^{\natural} \longrightarrow \operatorname{Pic}_{X / S}
$$

We wish to define a certain subfunctor of $\mathrm{Pic}_{X / S}^{\natural}$ which will play the role of "identity component" and which will enjoy good representability properties. We adopt the following definition: 
Definition 3.8. Let $\operatorname{Pic}_{X / S}^{\natural, 0}$ be the fppf abelian sheaf on the category of $S$-schemes given by

$$
\operatorname{Pic}_{X / S}^{\natural, 0}:=\operatorname{Pic}_{X / S}^{\natural} \times \operatorname{Pic}_{X / S} \operatorname{Pic}_{X / S}^{0} .
$$

Here, $\mathrm{Pic}_{X / S}^{0}$ denotes the identity component of the group functor $\mathrm{Pic}_{X / S}$ (whose fibers are representable; see [Liu et al. 2004, page 459] and compare [Bosch et al. 1990, page 233]). Alternately, $\mathrm{Pic}_{X / S}^{0}$ the open subfunctor of $\mathrm{Pic}_{X / S}$ classifying line bundles of partial degree zero on each irreducible component of every geometric fiber [Bosch et al. 1990, Section 9.2, Corollary 13].

Theorem 3.9. Let $f: X \rightarrow S$ be a proper relative curve and suppose that the greatest common divisor of the geometric multiplicities of the irreducible components of the closed fiber $X_{k}$ of $X$ is 1 . Then $\mathrm{Pic}_{X / S}^{\natural, 0}$ is a smooth $S$-scheme and there is a short exact sequence of smooth group schemes over $S$

$$
0 \longrightarrow f_{*} \omega_{X / S} \longrightarrow \mathrm{Pic}_{X / S}^{\natural, 0} \longrightarrow \mathrm{Pic}_{X / S}^{0} \longrightarrow 0 \text {. }
$$

To prove Theorem 3.9, we will first construct (3-12) as an exact sequence of fppf abelian sheaves. By work of Raynaud [1970, Theorem 8.2.1] (or [Bosch et al. 1990, Section 9.4, Theorem 2]), the hypotheses on $X$ imply that $\mathrm{Pic}_{X / S}^{0}$ is a separated $S$-group scheme which is smooth by [Bosch et al. 1990, Section 8.4, Proposition 2]. On the other hand, our hypotheses ensure that $X$ is cohomologically flat in dimension zero, whence $f_{*} \omega_{X / S}$ is a vector group (in particular, it is smooth and separated) by Corollary 3.4. A straightforward descent argument will complete the proof.

We will begin by constructing the exact sequence (3-12). Fix an $S$-scheme $T$ and consider the natural map (3-11). The kernel of this map consists of all isomorphism classes represented by pairs of the form $\left(0_{X_{T}}, \nabla\right)$, where $\nabla$ is a regular connection on $\mathrm{O}_{X_{T}}$ over $T$. By the Leibnitz rule, $\nabla$ is determined up to isomorphism by the value $\nabla(1) \in \Gamma\left(X_{T}, \omega_{X_{T} / T}\right)$. Since two pairs $\left(\mathcal{O}_{X_{T}}, \nabla\right)$ and $\left(\mathcal{O}_{X_{T}}, \nabla^{\prime}\right)$ are isomorphic precisely when there is a unit $u \in \Gamma\left(X_{T}, O_{X_{T}}^{\times}\right)$satisfying

$$
\nabla(1)=\nabla^{\prime}(1)+u^{-1} \cdot d_{T} u
$$

we see that the kernel of (3-11) is naturally identified with $H^{0}\left(X_{T}, \omega_{X_{T} / T}\right)$ modulo the image of the map

$$
d_{T} \log : H^{0}\left(X_{T}, \mathcal{O}_{X_{T}}^{\times}\right) \longrightarrow H^{0}\left(X_{T}, \omega_{X_{T} / T}\right)
$$

that sends a global section $u$ of $\mathcal{O}_{X_{T}}$ to $u^{-1} \cdot d_{T} u$. Since pushforward by the base change $f_{T *}$ of $f$ is left exact, we know that $f_{T *} \mathbb{O}_{X_{T}}^{\times}$is a subsheaf of $f_{T_{*}} \mathrm{O}_{X_{T}}$. By 
[Raynaud 1970, Théorème 7.2.1], the hypotheses on $X$ ensure that $f$ is cohomologically flat, so $f_{T *} \mathcal{O}_{X_{T}} \simeq \mathrm{O}_{T}$. Since $d_{T}$ annihilates $\Gamma\left(T, \mathrm{O}_{T}\right)$, we conclude that the map (3-13) is zero.

We thus arrive at a short exact sequence of abelian groups

$$
0 \longrightarrow H^{0}\left(X_{T}, \omega_{X_{T} / T}\right) \longrightarrow P_{X / S}^{\natural}(T) \longrightarrow P_{X / S}(T)
$$

that is easily seen to be functorial in $T$. In order to construct the exact sequence of fppf abelian sheaves (3-12), we need to extend (3-14). To do this, we use Čech theory to interpret (3-14) as part of a long exact sequence of cohomology groups.

Consider the two-term complex (in degrees 0 and 1) $d_{T} \log : \mathbb{O}_{X_{T}}^{\times} \rightarrow \omega_{X_{T} / T}$ given by sending a section $u$ of $0_{X_{T}}^{\times}$to $u^{-1} \cdot d_{T} u$; we will denote this complex by $\omega_{X_{T} / T}^{\times, \bullet}$. The evident short exact sequence of complexes

$$
0 \longrightarrow \omega_{X_{T} / T}[-1] \longrightarrow \omega_{X_{T} / T}^{\times, \bullet} \longrightarrow 0_{X_{T}}^{\times} \longrightarrow 0
$$

yields (since $d_{T} \log : H^{0}\left(X_{T}, \mathcal{O}_{X_{T}}^{\times}\right) \rightarrow H^{0}\left(X_{T}, \omega_{X_{T} / T}\right)$ is the zero map) a long exact sequence in hypercohomology that is clearly functorial in $T$ :

$$
\begin{aligned}
0 \longrightarrow H^{0}\left(X_{T}, \omega_{X_{T} / T}\right) \longrightarrow & \mathbf{H}^{1}\left(X_{T}, \omega_{X_{T} / T}^{\times, \bullet}\right) \\
& \longrightarrow H^{1}\left(X_{T}, O_{X_{T}}^{\times}\right) \stackrel{d_{T} \log }{\longrightarrow} H^{1}\left(X_{T}, \omega_{X_{T} / T}\right) .
\end{aligned}
$$

Lemma 3.10. For affine $T$, the exact sequence (3-14) is identified with the first three terms of (3-15) in a manner that is functorial in $T$.

Proof. By [EGA III 1961, Section 12.4, pp. 406-407], there is a natural identification of derived-functor (hyper)cohomology with Čech (hyper)cohomology which is $\delta$-functorial in degrees 0 and 1 . We thus have a natural identification of (3-15) with the corresponding exact sequence of Čech (hyper)cohomology groups, so it suffices to interpret (3-14) Čech-theoretically in a manner that is natural in $T$.

For $(\mathscr{L}, \nabla)$ representing a class in $P_{X / S}^{\natural}(T)$, let $\left\{U_{i}\right\}$ be a Zariski open cover of $X_{T}$ that trivializes $\mathscr{L}$, and denote by $f_{i j} \in \Gamma\left(U_{i} \cap U_{j}, \mathcal{O}_{X_{T}}^{\times}\right)$the transition functions. Because of the Leibnitz rule, $\left.\nabla\right|_{U_{i}}$ is determined by a unique "connection form" $\omega_{i} \in \Gamma\left(U_{i}, \omega_{X_{T} / T}\right)$, and the relation

$$
\omega_{i}-\omega_{j}=f_{i j}^{-1} \cdot d_{T} f_{i j}
$$

holds on $U_{i} \cap U_{j}$. We thus obtain a Čech 1-hypercocycle for the complex $\omega_{X_{T} / T}^{\times, \bullet}$ :

$$
\left(\left\{f_{i j}\right\},\left\{\omega_{i}\right\}\right) \in C^{1}\left(\left\{U_{i}\right\}, \omega_{X_{T} / T}^{\times, \bullet}\right):=C^{1}\left(\left\{U_{i} \cap U_{j}\right\}, \mathcal{O}_{X_{T}}^{\times}\right) \oplus C^{0}\left(\left\{U_{i}\right\}, \omega_{X_{T} / T}\right) .
$$


It is straightforward to check that any two such trivializations over open covers $\left\{U_{i}\right\}$ and $\left\{V_{j}\right\}$ yield hyper 1-cocycles which differ by a hyper coboundary when viewed as hyper 1-cocycles for the common refining open cover $\left\{U_{i} \cap V_{j}\right\}$, and likewise that two different representatives of the same isomorphism class in $P_{X / S}^{\natural}(T)$ yield hyper 1 -cocycles that differ by a hyper-coboundary (after passing to a common refining cover of the associated cocycles). We therefore obtain a well defined Čech hypercohomology class. This procedure is easily reversed, and so we have a bijection

$$
P_{X / S}^{\natural}(T) \simeq \check{\mathbf{H}}^{1}\left(X_{T}, \omega_{X_{T} / T}^{\times, \bullet}\right) .
$$

To check that this is in fact a homomorphism of abelian groups that is functorial in $T$ is straightforward (albeit tedious).

We identify $P_{X / S}(T)$ with $\check{H}^{1}\left(X_{T}, \mathcal{O}_{X_{T}}^{\times}\right)$in the usual way, by sending a class represented by $\mathscr{L}$ to the 1-cocycle $\left\{f_{i j}\right\}$ given by the transition functions associated to a trivializing open cover $\left\{U_{i}\right\}$ and choice of trivializations of $\left.\mathscr{L}\right|_{U_{i}}$. Similarly, we use the natural isomorphism of $H^{0}\left(X_{T}, \omega_{X_{T} / T}\right)$ with $\check{H}^{0}\left(X_{T}, \omega_{X_{T} / T}\right)$, and we thus obtain a functorial diagram of homomorphisms of abelian groups

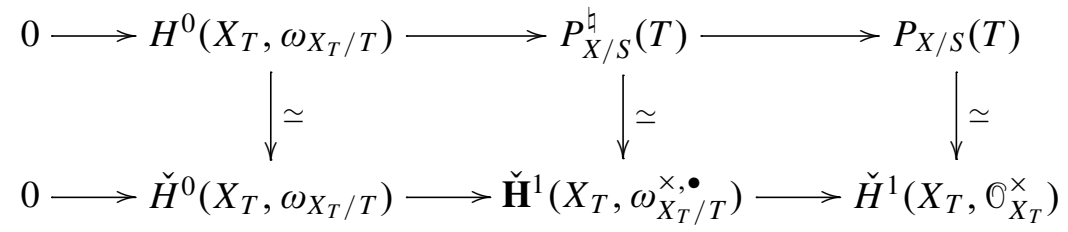

That this diagram commutes is easily verified by appealing to the explicit descriptions of the maps involved.

By Raynaud's critère de platitude cohomologique [Raynaud 1970, Théorème 7.2.1], our hypotheses $X$ ensure that $f$ is cohomologically flat in dimension zero. Thus, due to Corollary 3.4 and the fact that the formation of $\omega_{X / S}$ commutes with any base change on $S$, for each $i \geq 0$ the fppf sheaf associated to functor on $S$ schemes

$$
T \rightsquigarrow H^{i}\left(X_{T}, \omega_{X_{T} / T}\right)
$$

is represented by the vector group $R^{i} f_{*} \omega_{X / S}$. By Lemma 3.10, we therefore have an exact sequence of fppf sheaves of abelian groups on the category of $S$-schemes whose first and last (nonzero) terms are smooth affine $S$-groups:

$$
0 \longrightarrow f_{*} \omega_{X / S} \longrightarrow \operatorname{Pic}_{X / S}^{\natural} \longrightarrow \operatorname{Pic}_{X / S} \longrightarrow R^{1} f_{*} \omega_{X / S}
$$

With (3-16) at hand, we can now prove Theorem 3.9.

Proof of Theorem 3.9. Consider the identity component $\mathrm{Pic}_{X / S}^{0}$ and the composition of its inclusion into $\mathrm{Pic}_{X / S}$ with the map of fppf sheaves $\mathrm{Pic}_{X / S} \rightarrow R^{1} f_{*} \omega_{X / S}$. We claim that this composite is the zero map. Indeed, by [Raynaud 1970, Exemples 
6.1.6 and Théorème 8.2.1] (or [Bosch et al. 1990, Section 9.4, Theorem 2]) and [Bosch et al. 1990, Section 8.4, Proposition 2], our hypotheses on $X$ ensure that $\mathrm{Pic}_{X / S}^{0}$ is a smooth and separated $S$-scheme, so the composite map

$$
\operatorname{Pic}_{X / S}^{0} \longrightarrow R^{1} f_{*} \omega_{X / S}
$$

is a map of $S$-group schemes. Since the generic fiber of $\mathrm{Pic}_{X / S}^{0}$ is an abelian variety and $R^{1} f_{*} \omega_{X / S}$ is affine over $S$, the closed kernel of (3-17) contains the generic fiber, and hence (3-17) is the zero map. Thus, the inclusion $\mathrm{Pic}_{X / S}^{0} \rightarrow \mathrm{Pic}_{X / S}$ factors through the image of (3-11). By pullback, we obtain a short exact sequence (3-12) of fppf abelian sheaves on the category of $S$-schemes. As we have observed, the leftmost term in (3-12) is a vector group (in particular it is a smooth and affine $S$-group), and the rightmost term is a smooth and separated $S$-group scheme. It follows from this by fppf descent, as in the proof of Proposition 2.6, that $\mathrm{Pic}_{X / S}^{\natural, 0}$ is a smooth and separated $S$-group scheme, and that we have a short exact sequence (3-12) of smooth and separated group schemes over $S$.

\section{Proof of the main theorem}

In this section, we prove Theorem 1.2, following the outline sketched in the introduction (in particular, we will keep our notation from that section). Throughout this section, we fix a proper relative curve $f: X \rightarrow S$ over $S=\operatorname{Spec} R$ which we suppose satisfies the hypotheses of Theorem 1.2. Note that these hypotheses ensure that Grothendieck's pairing on component groups for $J_{K}$ is perfect, by Proposition 2.8. In particular, there is a natural short exact sequence of smooth $S$-groups:

$$
0 \longrightarrow \omega_{J} \longrightarrow \mathscr{E} x \operatorname{trig}_{S}\left(J, \mathbf{G}_{m}\right) \longrightarrow \widehat{J}^{0} \longrightarrow 0 \text {. }
$$

We begin our proof of Theorem 1.2 by constructing a canonical map of short exact sequences of smooth $S$-group schemes

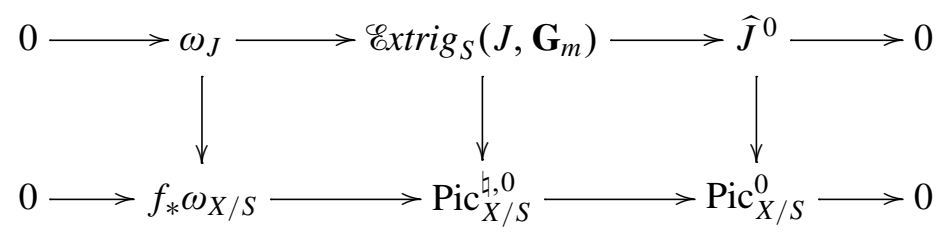

which we do in three steps.

Step 1. We initially suppose there exists a rational point $x \in X_{K}(K)$ and will later explain how to reduce the general case to this one. Associated to $x$ is the usual Albanese mapping $j_{x, K}: X_{K} \rightarrow J_{K}$ given by the functorial recipe

$$
y \mapsto \mathcal{O}(y) \otimes \mathcal{O}(x)^{-1} .
$$


Letting $i: X^{\mathrm{sm}} \hookrightarrow X$ denote the $S$-smooth locus of $f: X \rightarrow S$ in $X$, we denote by $j_{x}: X^{\mathrm{sm}} \rightarrow J$ the morphism obtained from $j_{x, K}$ by the Néron mapping property of $J$. By abuse of notation, we will also write $j_{x}$ for any base change of $j_{x}$. For each smooth and affine $S$-scheme $T$, we will show that "pullback along $j_{x}$ " yields a commutative diagram of exact sequences of abelian groups

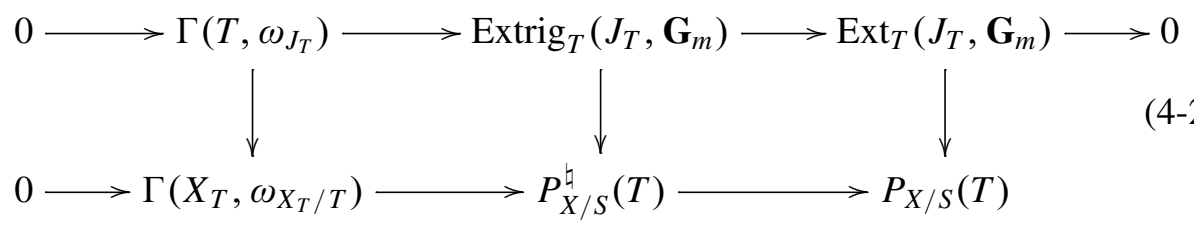

that is functorial in $T$. To do this, we will need to apply the following lemma with $U=X^{\mathrm{sm}}$; that this choice of $U$ satisfies the hypotheses of the lemma crucially uses our hypothesis that the closed fiber of $X$ is generically smooth.

Lemma 4.1. Let $U$ be any open subscheme of $X$ whose complement in $X$ consists of points of codimension at least 2. For each smooth $S$-scheme $T$, pushforward along $i_{T}: U_{T} \rightarrow X_{T}$ yields a natural isomorphism of short exact sequences of abelian groups

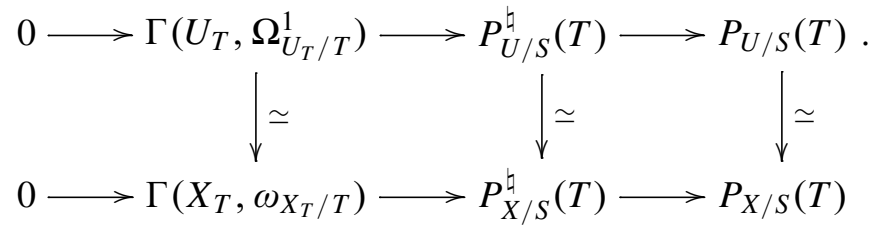

Proof. To minimize notation, we will simply write $i$ for $i_{T}$. Since the dualizing sheaf is compatible with étale localization, it suffices to show that for any pair $(\mathscr{L}, \nabla)$ consisting of a line bundle $\mathscr{L}$ on $X_{T}$ with regular connection $\nabla$ over $T$, the canonical commutative diagram

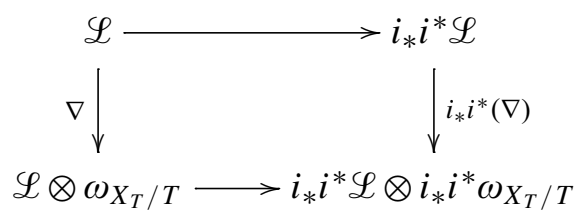

has horizontal arrows that are isomorphisms. By hypothesis, $X$ is normal and the complement of $U$ in $X$ consists of points of codimension at least two. Since $T \rightarrow S$ is smooth, the base change $X_{T}$ is also normal and the complement of $U_{T}$ in $X_{T}$ has codimension at least 2 (see part (ii) of the corollary to Theorem 23.9 and Theorem 15.1 in [Matsumura 1989]). As $\mathscr{L}$ is locally free, it follows that the top horizontal map of (4-3) is an isomorphism. By Lemma 3.2 the canonical map $\omega_{X / S} \rightarrow i_{*} i^{*} \omega_{X / S}$ is an isomorphism; since this map and the sheaves in question 
are compatible with base change, we conclude that the bottom horizontal arrow in (4-3) is also an isomorphism.

Remark 4.2. Note that Lemma 4.1 is generally false if the complement of $U$ in $X$ has codimension strictly less than 2 .

We deduce from Lemma 4.1 applied to $U:=X^{\text {sm }}$ that it suffices to construct (4-2) with $X$ replaced by $U$ in the bottom row. Note that since $U_{T}$ is $T$-smooth, the notions of regular connection and connection coincide (see Proposition 3.1). Thus, we wish to associate to any element of $\operatorname{Extrig}\left(J_{T}, \mathbf{G}_{m}\right)$ an invertible sheaf on $U_{T}$ with connection over $T$ in a manner that is Zariski-local on (and functorial in) $T$, and so globalizes from the case of affine $T$. To do this, we proceed as follows.

Fix a choice $\tau$ of generator for $\omega_{\mathbf{G}_{m}}$ and (functorially) identify $\operatorname{Extrig}_{T}\left(J_{T}, \mathbf{G}_{m}\right)$ with $\mathbf{E}_{\tau}\left(J_{T}\right)(T)$ via Lemma 2.1. Let $(E, \eta)$ be a representative of a class in $\mathbf{E}_{\tau}\left(J_{T}\right)(T)$. Viewing $E$ as a $\mathbf{G}_{m}$-torsor over $J_{T}$, we choose a Zariski open cover $\left\{V_{i}\right\}$ of $J_{T}$ and local sections $s_{i}: V_{i} \rightarrow E$ to the projection $E \rightarrow J_{T}$ that trivialize $E$ over $V_{i}$. Set $\omega_{i}:=s_{i}^{*} \eta \in \Gamma\left(V_{i}, \Omega_{J_{T} / T}^{1}\right)$ and let $\mathscr{L}$ be the invertible sheaf on $J_{T}$ corresponding to the $\mathbf{G}_{m}$-torsor $E$. There are two canonical ways to associate transition functions to $\mathscr{L}$ and the sections $s_{i}$ depending on whether we consider the section $s_{i}-s_{j}: V_{i} \cap V_{j} \rightarrow \mathbf{G}_{m}$ or its inverse $s_{j}-s_{i}$. However, since any two choices of $\tau$ differ by multiplication by \pm 1 , there is a unique choice $f_{i j}: V_{i} \cap V_{j} \rightarrow \mathbf{G}_{m}$ with the property that $f_{i j}^{*} \tau=f_{i j}^{-1} d f_{i j}$ (interpreting $f_{i j}$ as a section of $\mathbf{G}_{m}$ over $V_{i} \cap V_{j}$ ), and we consistently make this choice of transition function.

Define

$$
\nabla_{i}:\left.\left.\mathscr{L}\right|_{V_{i}} \longrightarrow \mathscr{L}\right|_{V_{i}} \otimes_{\mathcal{O}_{V_{i}}} \Omega_{V_{i} / T}^{1}
$$

by $\nabla_{i}\left(t s_{i}\right):=t s_{i} \otimes \omega_{i}+s_{i} \otimes d t$ for any section $t$ of $\mathcal{O}_{V_{i}}$. Using the definition of $\omega_{i}$ and the fact that $\eta$ pulls back to $\tau$ on $\mathbf{G}_{m}$, it is straightforward to check that

$$
\omega_{i}-\omega_{j}=f_{i j}^{*} \tau=f_{i j}^{-1} d f_{i j}
$$

(by our choice of $\left.f_{i j}\right)$ in $\Gamma\left(V_{i} \cap V_{j}, \Omega_{J_{T} / T}^{1}\right)$ and hence that the $\nabla_{i}$ uniquely glue to give a connection $\nabla$ on $\mathscr{L}$ over $T$. By passing to a common refining open cover, one checks that any other choice of trivialization $\left(V_{i^{\prime}}^{\prime}, s_{i^{\prime}}^{\prime}\right)$ yields the same connection on $\mathscr{L}$, so the pair $(\mathscr{L}, \nabla)$ is independent of our choices of cover $\left\{V_{i}\right\}$ and sections $\left\{s_{i}\right\}$.

By pullback along $j_{x}: U_{T} \rightarrow J_{T}$, we thus obtain a line bundle on $U_{T}$ with a connection. If $\left(E^{\prime}, \eta^{\prime}\right)$ is another choice of representative for the same class in $\mathbf{E}_{\tau}\left(J_{T}\right)(T)$ then by definition there is an isomorphism of extensions $\varphi: E \rightarrow E^{\prime}$ with the property that $\varphi^{*} \eta^{\prime}=\eta$. One easily checks that $\varphi$ induces an isomorphism of the invertible sheaves on $J_{T}$ with connection corresponding to $(E, \eta)$ and to $\left(E^{\prime}, \eta^{\prime}\right)$, and hence that we have a well defined map of sets $\mathbf{E}_{\tau}\left(J_{T}\right)(T) \rightarrow P_{U / S}^{\natural}(T)$ which is readily seen to be functorial in $T$. That this map is in fact a homomorphism of abelian groups follows easily from the definition using the description of the group 
law on $\mathbf{E}_{\tau}\left(J_{T}\right)(T)$ as in Section 2. By Lemma 2.1, we thus obtain a homomorphism of abelian groups

$$
\operatorname{Extrig}_{T}\left(J_{T}, \mathbf{G}_{m}\right) \longrightarrow P_{U / S}^{\natural}(T)
$$

that is functorial in $T$. It is straightforward to check that this map is moreover independent of our initial choice of $\tau$ (but may a priori depend on our choice of rational point $x$ ) and so provides the desired functorial map.

We similarly define $\operatorname{Ext}_{T}\left(J_{T}, \mathbf{G}_{m}\right) \rightarrow P_{U / S}(T)$ by associating to an extension $E$ of $J_{T}$ by $\mathbf{G}_{m}$ the pull back along $j_{x}: U_{T} \rightarrow J_{T}$ of the line bundle $\mathscr{L}$ on $J_{T}$ obtained by viewing $E$ as a $\mathbf{G}_{m}$-torsor over $J_{T}$. This is readily seen to be a homomorphism of abelian groups (using Baer sum on $\operatorname{Ext}_{T}\left(J_{T}, \mathbf{G}_{m}\right)$ ) and is obviously functorial in $T$.

Finally, we define $\Gamma\left(T, \omega_{J_{T}}\right) \rightarrow \Gamma\left(U_{T}, \Omega_{U_{T} / T}^{1}\right)$ as follows. By [Bosch et al. 1990, Section 4.1, Proposition 1], any global section $\omega_{0}$ of $\omega_{J_{T}}=e_{T}^{*} \Omega_{J_{T} / T}^{1}$ can be uniquely propagated to an invariant differential form $\omega$ on $J_{T}$ over $T$ satisfying $e_{T}^{*} \omega=\omega_{0}$. Pulling $\omega$ back along $j_{x}: U_{T} \rightarrow J_{T}$, we obtain a section of $\Omega_{U_{T} / T}^{1}$ over $U_{T}$. This association is clearly a homomorphism and functorial in $T$.

We thus obtain (via Lemma 4.1) a diagram (4-2) with all maps homomorphisms of abelian groups, functorially in smooth affine $S$-schemes $T$. That this diagram commutes follows immediately from the explicit definition of all the maps involved (morally, each vertical map is simply "pullback by $j_{x}$ ").

Step 2. Passing from (4-2) to the corresponding diagram of associated fppf sheaves and recalling the construction of the exact sequence of fppf sheaves (3-16) in Section 3, we obtain a commutative diagram of fppf sheaves of abelian groups

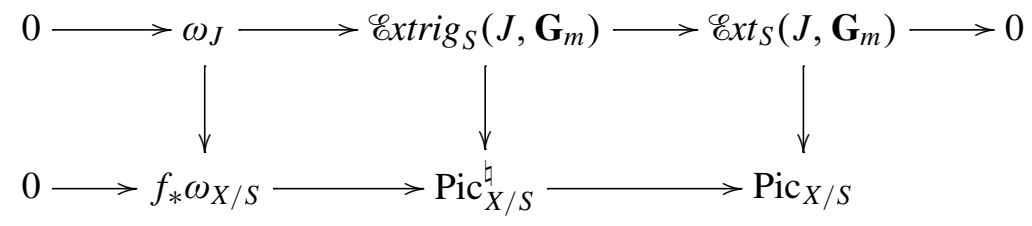

From Proposition 2.8, we thus deduce the following commutative diagram of fppf abelian sheaves on smooth $S$-schemes with each term in the top row a smooth $S$-group:

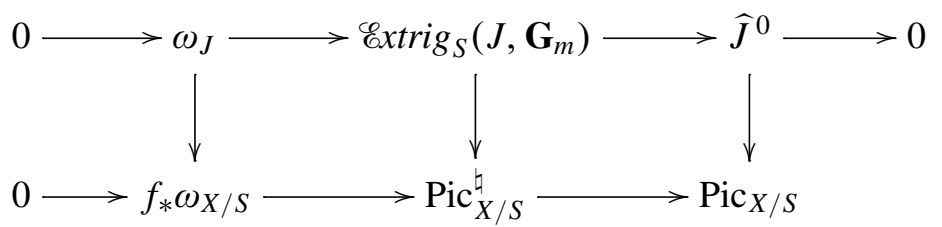

Since the map $\widehat{J}^{0} \rightarrow \mathrm{Pic}_{X / S}$ is homomorphism of group functors (on smooth $S$ schemes) and $\widehat{J}^{0}$ has connected fibers, for topological reasons this map necessarily 
factors through the open subfunctor $\mathrm{Pic}_{X / S}^{0}$ (thinking of $\mathrm{Pic}_{X / S}^{0}$ as the union of all identity components of the fibers of $\mathrm{Pic}_{X / S}$ [EGA IV 3 1966, Corollaire 15.6.5, p. 238] and arguing fiber-by-fiber). By the definition of $\operatorname{Pic}_{X / S}^{\natural, 0}$ (Definition 3.8) as a fiber product, we thus have a commutative diagram of fppf abelian sheaves on smooth $S$-schemes

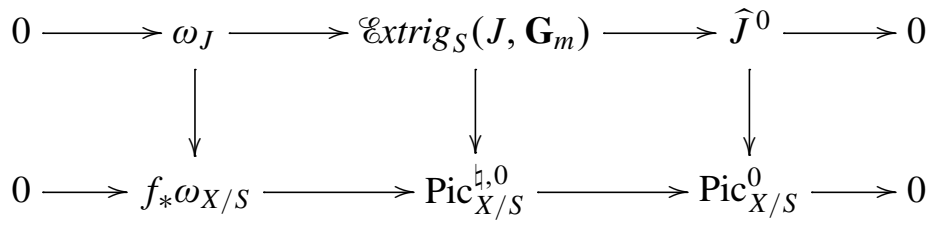

Step 3. By Proposition 2.6 and Theorem 3.9, both rows of (4-5) are short exact sequences of smooth $S$-group schemes, and we claim that the commutative diagram (4-5) of fppf sheaves on smooth $S$-schemes can be enhanced to a corresponding commutative diagram of maps between smooth group schemes over $S$. Indeed, this follows from Yoneda's lemma, which ensures that the natural "restriction to the smooth site" map

$$
\operatorname{Hom}_{S}(\mathscr{F}, \mathscr{G}) \rightarrow \operatorname{Hom}_{S_{\mathrm{sm}}}(\mathscr{F}, \mathscr{G})
$$

is bijective for any fppf abelian sheaves $\mathscr{F}, \mathscr{G}$ on $S$-schemes with $\mathscr{F}$ represented by a smooth $S$-group scheme.

We have therefore constructed (4-1) using our initial choice of rational point $x \in X_{K}(K)$. If $x^{\prime}$ is any other choice of rational point, we claim that the resulting maps (4-1) obtained from $x$ and $x^{\prime}$ coincide. Since $j_{x, K}, j_{x^{\prime}, K}: X_{K} \rightarrow J_{K}$ differ by a translation on $J_{K}$, it is enough to show that for any translation $\tau: J_{K} \rightarrow J_{K}$, the induced map

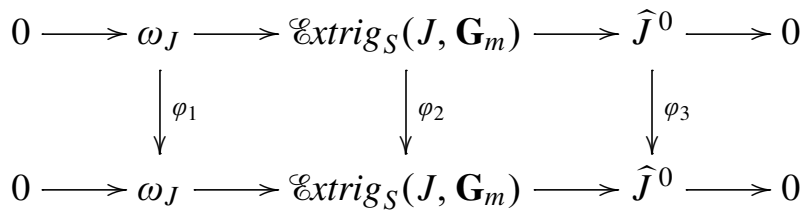

(using the Néron mapping property) is the identity map. Since each term in the bottom row is separated and each term in the top row is flat, whether or not (4-6) coincides with the identity map may be checked on generic fibers. Now $\tau^{*}: \omega_{J_{K}} \rightarrow \omega_{J_{K}}$ is the identity map as $\omega_{J_{K}}$ is identified with the sheaf of (translation) invariant differentials [Bosch et al. 1990, Section 4.2 Proposition 1]. That $\tau^{*}: \widehat{J}_{K} \rightarrow \widehat{J}_{K}$ is the identity is well known, and follows from the fact the line-bundles classified by $\widehat{J}_{K}:=\mathrm{Pic}_{J_{K} / K}^{0}$ are translation invariant (or equivalently 
that the classes in $\mathscr{E} x t_{K}\left(J_{K}, \mathbf{G}_{m}\right)$ are primitive $) .{ }^{5}$ Thus $\varphi_{1}=\varphi_{3}=\mathrm{id}$ so on $K$-fibers, $\varphi_{3}-$ id uniquely factors through a map $\widehat{J}_{K} \rightarrow \omega_{J_{K}}$ which takes the identity to the identity. As any map from an abelian variety to a vector group is constant, we conclude that $\varphi_{3}-$ id is identically zero on $K$-fibers, and hence that $\varphi_{3}=$ id as well. Thus, the map (4-1) which we have constructed is independent of the choice of rational point $x \in X_{K}(K)$.

In the general case when $X_{K}(K)$ may be empty, we proceed as follows. Denote by $Y$ any one of the three schemes occurring in the top row of (4-1) and by $Z$ the corresponding scheme in the bottom row. We first claim that we have a canonical map $Y_{K} \rightarrow Z_{K}$. Indeed, $X_{K}$ has a $K^{\prime}$-rational point for some finite Galois extension $K^{\prime}$ of $K$, and we may use this point to define a $K^{\prime}$-map $Y_{K^{\prime}} \rightarrow Z_{K^{\prime}}$ as we have explained. Since this map is independent of the choice of $K^{\prime}$-rational point by what we have said above, via Galois descent we have a canonical $K$-map $\varphi_{K}: Y_{K} \rightarrow Z_{K}$ as claimed.

We now appeal to the following general lemma.

Lemma 4.3. Fix an integral scheme $T$ with generic point $\eta$ and let $Y \rightarrow T$ and $Z \rightarrow T$ be any flat $T$-schemes, with $Z$ separated over $T$. Suppose given a map $\varphi_{\eta}: Y_{\eta} \rightarrow Z_{\eta}$. Then there is at most one extension of $\varphi_{\eta}$ to a $T$-map $\varphi: Y \rightarrow Z$, and $\varphi$ exists if and only if the schematic closure in $Y \times_{T} Z$ of the graph of $\varphi_{\eta}$ maps isomorphically onto $Y$ by the first projection. In particular, $\varphi$ exists if and only if there is an fpqc morphism $T^{\prime} \rightarrow T$ and a map $\varphi^{\prime}: Y_{T^{\prime}} \rightarrow Z_{T^{\prime}}$ with $\varphi_{\eta^{\prime}}^{\prime}=\varphi_{\eta^{\prime}}$ where $\eta^{\prime}=T^{\prime} \times_{T} \eta$.

Proof. The proof of Lemma 4.3 proceeds via standard arguments with schematic closures of graphs; due to lack of a reference, we sketch how this goes. The uniqueness of an extension is clear, as $T$ is integral, $Z$ is separated over $T$, and $Y$ is $T$-flat. For existence, we proceed as follows. Let $\Gamma \subseteq Y \times_{T} Z$ be the schematic closure in $Y \times_{T} Z$ of the graph $\Gamma_{\varphi_{\eta}} \subseteq Y_{\eta} \times_{\eta} Z_{\eta}$ of $\varphi_{\eta}$, and note that $\Gamma_{\eta}=\Gamma_{\varphi_{\eta}}$ as $Z$ is $T$-separated. Now if the first projection $\Gamma \rightarrow Y$ is an isomorphism then it is clear that $\varphi_{\eta}$ extends to a $T$-morphism. Conversely, given $\varphi: Y \rightarrow Z$ extending $\varphi_{\eta}$ and denoting by $\Gamma_{\varphi}$ the graph of $\varphi$, we claim that necessarily $\Gamma=\Gamma_{\varphi}$. Indeed,

${ }^{5}$ More precisely, for any abelian variety $A$ over $K$ we have a homomorphism of group functors

$$
\phi: \operatorname{Pic}_{A / K} \rightarrow \operatorname{Hom}\left(A, \operatorname{Pic}_{A / K}^{0}\right)
$$

given functorially on $K$-schemes $T$ by sending a line bundle $\mathscr{L}$ on $A_{T}$ to the map $x \mapsto \tau_{x}^{*} \mathscr{L} \otimes$ $\mathscr{L}^{-1}$ with $\tau_{x}$ translation by a $T$-point $x$ of $A_{T}$. Since $A$ and $\mathrm{Pic}_{A / K}^{0}$ are projective, Grothendieck's theory of Hom-schemes ensures that $\operatorname{Hom}\left(A, \mathrm{Pic}_{A / K}^{0}\right)$ is a finite-type $K$-scheme which we claim is étale. Working over $\bar{K}$, our claim follows from the fact that there are no nonzero liftings to $K[\epsilon]$ of the zero map $A \rightarrow \mathrm{Pic}_{A / K}^{0}$ (due to [Mumford et al. 1994, Theorem 6.1]), so the tangent space of $\operatorname{Hom}\left(A, \operatorname{Pic}_{A / K}^{0}\right)$ at the origin is zero. Again passing to $\bar{K}$, we conclude that the group map $\phi$ maps connected components of $\mathrm{Pic}_{A / K}$ to individual points, so in particular restricts to the zero map on the connected component of the identity $\mathrm{Pic}_{X / K}^{0}$. 
the canonical closed immersion $\Gamma \rightarrow Y \times_{T} Z$ factors through a closed immersion $\Gamma \rightarrow \Gamma_{\varphi}$ as $\Gamma_{\varphi}$ is closed in $Y \times_{T} Z$ (due to $T$-separatedness of $Z$ ) and contains $\Gamma_{\varphi_{\eta}}$. Since the closed immersion $\Gamma \rightarrow \Gamma_{\varphi}$ is an isomorphism over $\eta$ (using that $\Gamma_{\eta} \simeq \Gamma_{\varphi_{\eta}}$ ) it must be an isomorphism, since $\Gamma_{\varphi_{\eta}}$ is dense in $\Gamma_{\varphi}$ as $\Gamma_{\varphi}$ is $T$-flat and $T$ is integral. We conclude that $\Gamma=\Gamma_{\varphi}$ maps isomorphically onto $Y$ via the first projection. Finally, whether or not the first projection $\Gamma \rightarrow Y$ is an isomorphism is insensitive to fpqc base change; since the formation of $\Gamma$ commutes with such base change (as $\eta \rightarrow T$ is quasicompact and separated), we deduce the last statement of the lemma.

Applying the lemma with $T=S=\operatorname{Spec}(R), Y, Z$ as above, and $T^{\prime}=\operatorname{Spec}\left(R^{\mathrm{sh}}\right)$ for $R^{\text {sh }}$ a strict henselization of $R$, we see that it remains to construct a $T^{\prime}$-morphism $Y_{T^{\prime}} \rightarrow Z_{T^{\prime}}$ recovering the base change of $\varphi_{K}$ to $K^{\mathrm{sh}}:=\operatorname{Frac}\left(R^{\mathrm{sh}}\right)$ on generic fibers. Now $X$ has generically smooth closed fiber, so $X_{K}$ has a $K^{\text {sh }}$-point. As our hypotheses on $X$ are unaltered by base change along local-étale extensions of discrete valuation rings (such as $R \rightarrow R^{\mathrm{sh}}$ ) and the formation of the top and bottom rows of (4-1) commute with such base change we may use this $K^{\text {sh }}$-point as in the construction of (4-1) to define the desired $T^{\prime}$-map $Y_{T^{\prime}} \rightarrow Z_{T^{\prime}}$. We conclude that $\varphi_{K}$ uniquely extends to an $S$-map, and thus we obtain (4-1) over $S$, as desired.

Now that we have constructed the canonical map of short exact sequences of smooth $S$-groups (4-1), we can show that it is an isomorphism. We reiterate here that only the construction of this map uses the hypothesis that the closed fiber of $X$ is generically smooth; as we will see below, the proof that (4-1) is an isomorphism requires only the weaker hypotheses of Raynaud's Theorem 1.1.

Proof of Theorem 1.2. By passing to a finite étale extension if necessary, we may assume that $X_{K}(K)$ is nonempty, and we select $x \in X_{K}(K)$ and use it to define (4-1). Note that since $X$ is normal with generically smooth closed fiber, $X$ satisfies the hypotheses of Theorem 1.1.

Consider the composite mapping

$$
\operatorname{Pic}_{X / S}^{0} \longrightarrow J^{0} \stackrel{\simeq}{\longrightarrow} \widehat{J}^{0}
$$

where the first map is deduced via the Néron mapping property from the canonical identification $J_{K}=\mathrm{Pic}_{X_{K} / K}^{0}$ and the second map is similarly obtained from the canonical principal polarization $J_{K} \rightarrow \widehat{J}_{K}$ induced by the $\Theta$-divisor [Milne 1986b, Section 6]. We claim that the composite $\widehat{J}^{0} \rightarrow \widehat{J}^{0}$ of (4-7) with the right vertical map of (4-1) coincides with negation on $\widehat{J}^{0}$. Since $\widehat{J}^{0}$ is flat and separated, it suffices to check this claim on generic fibers, so we wish to show that the map $\operatorname{Pic}^{0}\left(j_{x, K}\right): \widehat{J}_{K} \rightarrow J_{K}$ is the negative of the inverse of the canonical principal polarization $J_{K} \rightarrow \widehat{J}_{K}$. This is the content of [Milne 1986b, Lemma 6.9]. It follows from Theorem 1.1 that the right vertical map of (4-1) is an isomorphism 
if and only if $X$ has rational singularities; in particular, this settles the "only if" direction of Theorem 1.2.

We henceforth suppose that $X$ has rational singularities and we wish to show that (4-1) is an isomorphism of exact sequences of smooth group schemes over $S$. By Theorem 1.1 and our discussion, we know that the right vertical map of (4-1) is an isomorphism, and we will "bootstrap" Raynaud's theorem using duality; more precisely, we will show that the left vertical map in (4-1) is dual to the map on Lie algebras obtained from (1-2) and must therefore be an isomorphism as well. Indeed, consider the dual of the map on Lie algebras obtained from (1-2):

$$
\mathscr{L} i e\left(J^{0}\right)^{\vee} \stackrel{\simeq}{\longrightarrow} \operatorname{Lie}\left(\operatorname{Pic}_{X / S}^{0}\right)^{\vee} .
$$

For any commutative group functor $G$ over $S$ with representable fibers, the canonical inclusion $G^{0} \hookrightarrow G$ induces an isomorphism on Lie algebras [Liu et al. 2004, Proposition 1.1(d)], so we obtain a natural isomorphism of $\mathscr{O}_{S}$-modules $\mathscr{L}_{i e}(J)^{\vee} \simeq$ $\mathscr{L i e}\left(\mathrm{Pic}_{X / S}\right)^{\vee}$. The canonical identification $R^{1} f_{*} \mathrm{O}_{X} \simeq \mathscr{L} i e\left(\mathrm{Pic}_{X / S}\right)$ ([Bosch et al. 1990, Section 8.4, Theorem 1] or [Liu et al. 2004, Proposition 1.3(b)]) then gives a natural isomorphism

$$
\mathscr{L i e}(J)^{\vee} \stackrel{\simeq}{\longrightarrow}\left(R^{1} f_{*} \mathrm{O}_{X}\right)^{\vee} .
$$

Using the canonical duality $\omega_{J} \simeq \mathscr{L i e}(J)^{\vee}$ (see [SGA3-1 1970, II, Section 4.11] or [Liu et al. 2004, Proposition 1.1(b)]) and Grothendieck duality (Proposition 3.3) yields a natural isomorphism of $\mathrm{O}_{S}$-modules

$$
\omega_{J} \stackrel{\simeq}{\longrightarrow} \operatorname{Lie}(J)^{\vee} \underset{(4-8)}{\simeq}\left(R^{1} f_{*} \mathrm{O}_{X}\right)^{\vee} \underset{(3-7)}{\simeq} f_{*} \omega_{X / S}
$$

and hence an isomorphism of the corresponding vector groups over $S$. We claim that the left vertical map in (4-1) coincides with the negative of (4-9). Since the source of both maps is flat and the target is separated over $S$, it suffices to check such agreement on generic fibers.

To do this, we consider the following diagram, in which we simply write $j$ for $j_{x, K}$ and $\varphi: J_{K} \rightarrow \widehat{J}_{K}$ for the canonical principal polarization:

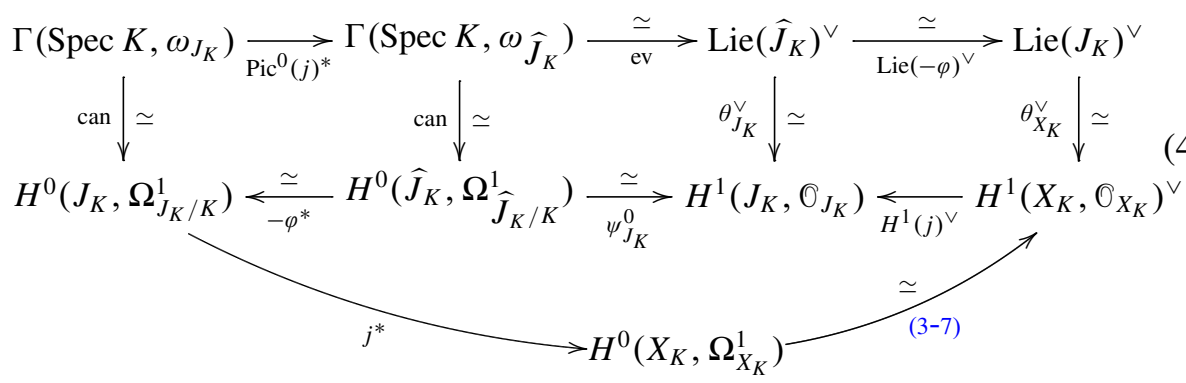


Here, $\psi_{J_{K}}^{0}$ is the usual duality (defined using the Künneth formula and the first Chern class of the Poincaré bundle [Berthelot et al. 1982, 5.1.3 and Lemme 5.1.4]; the map ev is the canonical evaluation pairing, and can is the canonical map obtained by extending sections of $\omega_{J_{K}}$ to invariant differential forms on $J_{K}$ [Bosch et al. 1990, Section 4.2, Propositions 1 and 2]. We claim that each of the three small squares in (4-10) commute, and that the bottom "sector" anticommutes. For the first square, this follows from the fact that the composite $\operatorname{Pic}^{0}(j) \circ(-\varphi): J \rightarrow J$ is the identity map [Milne 1986b, Lemma 6.9], together with the fact that the canonical map can is functorial. The same reasoning shows that the third square commutes, using the functoriality of the identification $\theta_{K}$ [Liu et al. 2004, Proposition 1.3(c)]. The commutativity of the middle square follows immediately from 5.1.1 and the proof of Théorème 5.1.6 in [Berthelot et al. 1982]. That the bottom sector region anticommutes is the content of [Coleman 1998, Theorem 5.1]. Note, as a particular consequence of these commutativity statements, that every map occurring in (4-10) is an isomorphism.

Using the functoriality in $J_{K}$ of the canonical duality $\omega_{J_{K}} \simeq \mathscr{L} i e\left(J_{K}\right)$ [Liu et al. 2004, Proposition 1.1(b)] and the agreement of $-\varphi^{-1}$ with $\operatorname{Pic}^{0}(j): \widehat{J}_{K} \rightarrow J$, as above, we conclude that the composite isomorphism $\Gamma\left(\operatorname{Spec} K, \omega_{J_{K}}\right) \rightarrow \operatorname{Lie}\left(J_{K}\right)^{\vee}$ along the top row of (4-10) is the canonical evaluation duality for $J_{K}$. Thus, on generic fibers, the map (4-9) is none other than the map induced by the top, right, and bottom-right edges in (4-10). But by definition, the left vertical map of (4-1) coincides with the composite of the left and bottom-left edges of (4-10) on generic fibers, and is thus the negative of (4-9), as claimed.

Now that we know that the left and right vertical maps in (4-1) are isomorphisms when $X$ has rational singularities, it follows that the same is true of all three vertical maps, as desired.

Remark 4.4. That (4-9) coincides with the left vertical map in (4-1) over generic fibers is essentially Theorem B.4.1 of [Conrad 2000]. We have chosen here to present a different proof because [Conrad 2000, Theorem B.4.1] rests upon knowing a priori that the natural pullback map $\Omega_{J_{K} / K}^{1} \rightarrow j_{*} \Omega_{X_{K} / K}^{1}$ is an isomorphism, while we prefer to deduce this fact as a corollary of our main result.

\section{Comparison of integral structures}

In this section, we use Theorem 1.2 to prove a comparison result for integral structures in de Rham cohomology. As usual, we fix a discrete valuation ring $R$ with field of fractions $K$.

Let $A_{K}$ be an abelian variety over $K$. It is well known that the Lie algebra of the universal extension $E\left(\widehat{A}_{K}\right)$ of the dual abelian variety $\widehat{A}_{K}$ is naturally isomorphic to the first de Rham cohomology of $A_{K}$ over $K$, compatibly with Hodge filtrations. 
Proposition 5.1. There is a canonical isomorphism of short exact sequences of finite dimensional $K$-vector spaces

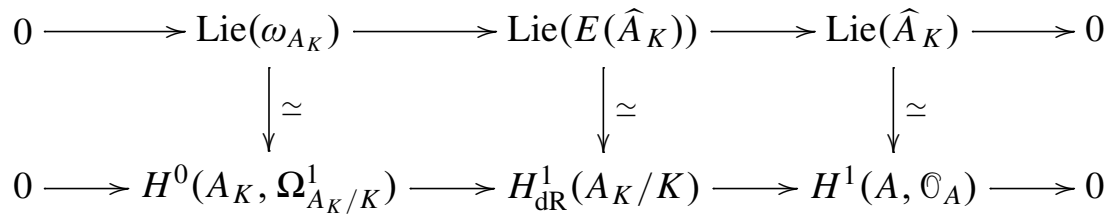

Proof. See [Mazur and Messing 1974, I, Section 4].

Note that since $\omega_{A_{K}}$ is a vector group, we have a canonical identification of $\operatorname{Lie}\left(\omega_{A_{K}}\right)$ with the global sections of $\omega_{A_{K}}$. We deduce from Proposition 5.1 and Proposition 2.3 the following corollary, which equips the de Rham cohomology of $A_{K}$ with a canonical integral structure.

Corollary 5.2. Let $A$ and $\widehat{A}$ be the Néron models over $R$ of $A_{K}$ and $\widehat{A}_{K}$, respectively, and let $\mathscr{E}(\widehat{A})$ be the canonical extension of $\widehat{A}$ (Definition 2.4). The sequence of Lie algebras

$$
0 \longrightarrow \operatorname{Lie}\left(\omega_{A}\right) \longrightarrow \operatorname{Lie}(\mathscr{E}(\widehat{A})) \longrightarrow \operatorname{Lie}(\widehat{A}) \longrightarrow 0
$$

associated to the canonical extension (2-6) of $\widehat{A}$ over $R$ is a short exact sequence of finite free $R$-modules that is contravariantly functorial in $K$-morphisms of abelian varieties $A_{K} \rightarrow B_{K}$ over $K$ and recovers the 3-term Hodge filtration of $H_{\mathrm{dR}}^{1}\left(A_{K} / K\right)$ after extending scalars to $K$. That is, (5-1) provides a canonical integral structure on the 3-term Hodge filtration of $H_{\mathrm{dR}}^{1}\left(A_{K} / K\right)$.

Proof. Each term in (2-6) is a smooth $S$-scheme; in particular the map

$$
\mathscr{E}^{x} \operatorname{trig}_{S}\left(A^{0}, \mathbf{G}_{m}\right) \rightarrow \widehat{A}
$$

is smooth [SGA3-1 1970, Exposé VI $\mathrm{B}_{\mathrm{B}}$, Proposition 9.2 vii]. Thus, by [Liu et al. 2004, Proposition 1.1(c)], applying the left exact functor Lie to (2-6) yields a short exact sequence of finite $R$-modules which are free by smoothness. Since any homomorphism of Néron models $A \rightarrow B$ induces a map on identity components $A^{0} \rightarrow B^{0}$, it follows from the Néron mapping property and the functoriality of the canonical extension (2-6) that (5-1) is contravariantly functorial in $K$-morphisms of abelian varieties $A_{K} \rightarrow B_{K}$ over $K$. Since the formation of Lie algebras commutes with the scalar extension $R \rightarrow K$, we deduce from Proposition 5.1 and the fact that $K$-fiber of (2-6) is the universal extension of $\widehat{A}_{K}$ by a vector group (see Remarks $2.5)$ that (5-1) recovers the Hodge filtration of $H_{\mathrm{dR}}^{1}\left(A_{K} / K\right)$ after extending scalars to $K$.

Remark 5.3. Supposing that Grothendieck's pairing (2-7) is right nondegenerate, so $\mathscr{E}^{\mathrm{x}} \operatorname{trig}_{S}\left(A, \mathbf{G}_{m}\right)$ is a smooth and separated $S$-scheme by Proposition 2.6, the 
natural map of short exact sequences (2-10) induces an isomorphism on associated exact sequences of Lie algebras by Lemma 2.10 and [Liu et al. 2004, Proposition $1.1(\mathrm{~d})]$.

For the remainder of this section, we suppose that $A_{K}=J_{K}$ is the Jacobian of a smooth proper and geometrically connected curve $X_{K}$ over $K$. Recall that the 3-term Hodge filtration

$$
0 \longrightarrow H^{0}\left(X_{K}, \Omega_{X_{K} / K}^{1}\right) \longrightarrow H_{\mathrm{dR}}^{1}\left(X_{K} / K\right) \longrightarrow H^{1}\left(X_{K}, \mathrm{O}_{X_{K}}\right) \longrightarrow 0
$$

is autodual with respect to the cup product pairing on $H_{\mathrm{dR}}^{1}\left(X_{K} / K\right)$ and is contravariantly and covariantly functorial in finite morphisms of smooth and proper curves $g: X_{K} \rightarrow X_{K}^{\prime}$ via pullback $g^{*}$ and pushforward $g_{*}$ of differentials. Attached to any such morphism, we have associated homomorphisms of abelian varieties

$$
\operatorname{Pic}^{0}(g): J_{K}^{\prime} \rightarrow J_{K} \quad \text { and } \quad \operatorname{Alb}(g): J_{K} \rightarrow J_{K}^{\prime}
$$

by Picard and Albanese functoriality (where $J_{K}^{\prime}$ is the Jacobian of $X_{K}^{\prime}$ ). The following proposition is well known, but we have been unable to find a proof in the literature so we include one here for the convenience of the reader.

Proposition 5.4. There is a canonical isomorphism of short exact sequences of $K$-vector spaces

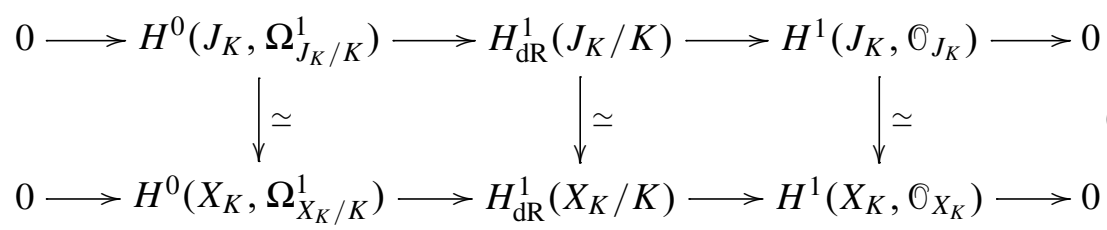

This isomorphism respects the autodualities of the top and bottom rows. Furthermore, for any finite morphism $g: X_{K} \rightarrow X_{K}^{\prime}$, the map (5-3) intertwines $\operatorname{Alb}(g)^{*}$ with $g^{*}$ and $\operatorname{Pic}^{0}(g)^{*}$ with $g_{*}$.

Proof. We first suppose that $X(K)$ is nonempty and select $x_{0} \in X(K)$. Let $j: X_{K} \rightarrow J_{K}$ be the associated Albanese morphism. By pullback along $j$, we obtain a morphism on de Rham cohomology that yields a commutative diagram (5-3). Clearly this map commutes with extension of $K$ (using the same $x_{0}$ ) and we claim that it is independent of our choice $x_{0}$. Each term in the Hodge filtration of $H_{\mathrm{dR}}^{1}\left(J_{K} / K\right)$ is clearly (the global sections of) a vector group over $K$; denoting any one of them by $V$ it suffices to show that the natural map $J_{K} \rightarrow \operatorname{Aut}_{K}(V)$ given by translations is the zero map. Since the target is affine of finite type over $K$ and the source is an abelian variety, this map factors through a section of the target and must therefore be identically zero, as claimed. It follows from Galois descent that we have a canonical map (5-3) even when $X(K)$ is empty. 
Let us denote by $H\left(J_{K}\right)$ (respectively $H\left(X_{K}\right)$ ) the 3-term exact sequence of $K$-vector spaces given by the top (respectively bottom) row of (5-3). By passing to an extension of $K$ if need be, we may suppose that $X_{K}(K)$ is nonempty and that (5-3) is given by pullback along an Albanese morphism $j: X_{K} \rightarrow J_{K}$ associated to some $x_{0} \in X_{K}(K)$. To show that (5-3) is an isomorphism, we will exploit the natural autodualities on $H\left(J_{K}\right)$ and $H\left(X_{K}\right)$. For this to be successful, it is essential to know that these dualities are compatible.

Lemma 5.5. The canonical autodualities of the short exact sequences $H\left(J_{K}\right)$ and $H\left(X_{K}\right)$ are compatible via $j^{*}$. That is, the following diagram commutes:

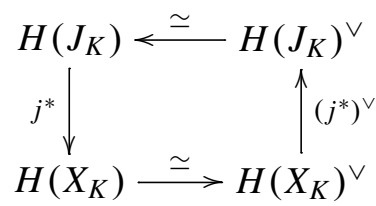

Proof. This is Theorem 5.1 of [Coleman 1998].

Continuing with the proof of Proposition 5.4, observe that the functoriality of the canonical identification $H^{1}\left(X_{K}, \mathrm{O}_{X_{K}}\right) \simeq \operatorname{Lie}\left(\mathrm{Pic}_{X_{K} / K}^{0}\right)$ yields a commutative diagram

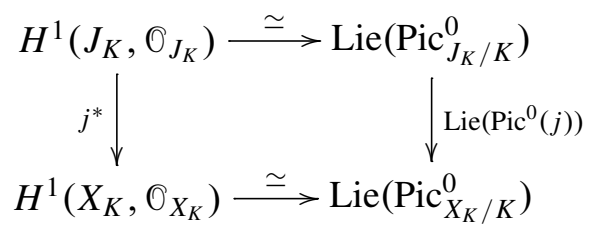

(see [Liu et al. 2004, Proposition 1.3(c)]). Due to [Milne 1986b, Lemma 6.9], the map $\operatorname{Pic}^{0}(j): \widehat{J}_{K} \rightarrow J_{K}$ is the negative of the inverse of the canonical principal polarization $J_{K} \rightarrow \widehat{J}_{K}$, so in particular it is an isomorphism. Thus, the map $j^{*}$ : $H^{1}\left(J_{K}, \mathcal{O}_{J_{K}}\right) \rightarrow H^{1}\left(X_{K}, \mathcal{O}_{X_{K}}\right)$ is an isomorphism. Taking $K$-linear duals and using the autoduality of $H\left(J_{K}\right)$ and $H\left(X_{K}\right)$, it follows from Lemma 5.5 that the map $j^{*}: H^{0}\left(J_{K}, \omega_{J_{K}}\right) \rightarrow H^{0}\left(X_{K}, \Omega_{X_{K} / K}^{1}\right)$ is also an isomorphism. We conclude that all three vertical maps of (5-3) are isomorphisms, as desired.

It remains to check our claims concerning the functoriality of (5-3) in finite morphisms of smooth proper and geometrically connected curves $g: X_{K} \rightarrow X_{K}^{\prime}$. Denote by $J_{K}^{\prime}$ the Jacobian of $X_{K}^{\prime}$ and by $j^{\prime}: X_{K}^{\prime} \rightarrow J_{K}^{\prime}$ the Albanese map attached to $g\left(x_{0}\right)$. Albanese functoriality gives a commutative diagram

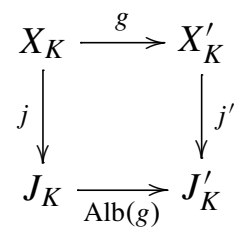


from which we easily obtain the commutative diagram of short exact sequences

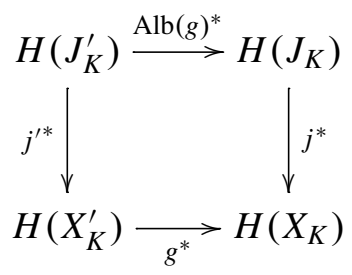

which shows that (5-3) intertwines the maps $g^{*}$ and $\operatorname{Alb}(g)^{*}$. Dualizing (5-5) and using Lemma 5.5 gives a commutative diagram

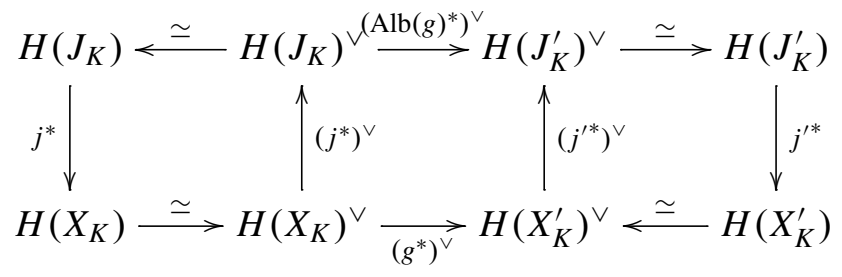

By [Cais 2009, Theorem 5.11(3)], the maps $g^{*}$ and $g_{*}$ are adjoint with respect to the cup-product pairing on $H_{\mathrm{dR}}^{1}\left(X_{K} / K\right)$, so the composite map on the bottom row of (5-6) coincides with $g_{*}$. We claim that $\operatorname{Alb}(g)^{*}$ and $\operatorname{Pic}^{0}(g)^{*}$ are adjoint with respect to the pairing on $H_{\mathrm{dR}}^{1}\left(J_{K} / K\right)$, so the top row of (5-6) coincides with $\operatorname{Pic}^{0}(g)^{*}$. By definition, the duality pairing on $H_{\mathrm{dR}}^{1}\left(J_{K} / K\right)$ is deduced from the natural perfect pairing

$$
H_{\mathrm{dR}}^{1}\left(J_{K} / K\right) \times H_{\mathrm{dR}}^{1}\left(\widehat{J}_{K} / K\right) \longrightarrow K
$$

(defined as in [Berthelot et al. 1982, Section 5]) by identifying the de Rham cohomology of $\widehat{J}_{K}$ with that of $J_{K}$ via the principal polarization $J_{K} \simeq \widehat{J}_{K}$. Now if $u: J_{K}^{\prime} \rightarrow J_{K}$ is any morphism with dual $\hat{u}: \widehat{J}_{K} \rightarrow \widehat{J}_{K}^{\prime}$, then the induced maps $u^{*}$ and $\hat{u}^{*}$ on de Rham cohomology are adjoint with respect to (5-7) by [Berthelot et al. 1982, 5.1.3.3]. Applying this to $u=\operatorname{Pic}^{0}(g)$, our claim that $\operatorname{Alb}(g)^{*}$ and $\operatorname{Pic}^{0}(g)^{*}$ are adjoint then follows from the assertion that the composite map

$$
J_{K} \stackrel{\simeq}{\varphi} \widehat{J}_{K} \stackrel{\widehat{\operatorname{Pic}^{0}(g)}}{\longrightarrow} \widehat{J}_{K}^{\prime} \underset{\varphi^{\prime}}{\simeq} J_{K}^{\prime}
$$

coincides with $\operatorname{Alb}(g)$, where $\varphi$ and $\varphi^{\prime}$ are the canonical principal polarizations. But this follows by applying the functor $\mathrm{Pic}^{0}$ to the diagram (5-4) and using the fact that $\operatorname{Pic}^{0}(j)$ and $\operatorname{Pic}^{0}\left(j^{\prime}\right)$ coincide with $-\varphi^{-1}$ and $-\varphi^{\prime-1}$, respectively, thanks to [Milne 1986b, Lemma 6.9].

Fix a proper flat and normal model $f: X \rightarrow S$ of $X_{K}$ over $S=\operatorname{Spec} R$, and denote by $\omega_{X / S}^{\bullet}$ the two-term $\mathscr{O}_{S}$-linear complex of $\mathscr{O}_{X}$-modules $d: \mathscr{O}_{X} \rightarrow \omega_{X / S}$ 
furnished by Proposition 3.1. We will say that $X$ is an admissible model of $X_{K}$ if $X$ has rational singularities and $f$ is cohomologically flat in dimension zero.

Define $H^{1}(X / R):=\mathbf{H}^{1}\left(X, \omega_{X / S}^{\bullet}\right)$. When $X$ is cohomologically flat, there is a natural short exact sequence of finite free $R$-modules

$$
0 \longrightarrow H^{0}\left(X, \omega_{X / S}\right) \longrightarrow H^{1}(X / R) \longrightarrow H^{1}\left(X, O_{X}\right) \longrightarrow 0
$$

whose scalar extension to $K$ is identified with the 3-term Hodge filtration exact sequence $H\left(X_{K}\right)$. Moreover, (5-8) is self-dual with respect to the usual cup-product autoduality of $H\left(X_{K}\right)$; see Proposition 5.8 of [Cais 2009]. By Theorem 5.11 of the same paper, when $X$ is admissible, the integral structure provided by (5-8) is canonical: this short exact sequence is independent of the choice of admissible model $X$ of $X_{K}$ and is both contravariantly and covariantly functorial via pullback and trace in finite $K$-morphisms $X_{K} \rightarrow X_{K}^{\prime}$ of curves having admissible models over $R$.

Via Corollary 5.2 and the identification of Hodge filtrations (5-3), when $X$ is admissible we thus have two canonical integral structures on the de Rham cohomology of $X_{K}$, and it is natural to ask how these $R$-lattices compare.

Corollary 5.6. With the notation and hypotheses of Theorem 1.2, when $X$ has rational singularities there is a canonical isomorphism of short exact sequences of finite free R-modules

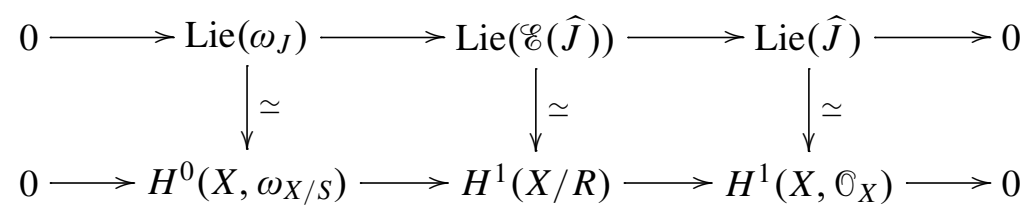

that recovers the identification (5-3) after extending scalars to $K$.

Remark 5.7. Let $g: X_{K} \rightarrow X_{K}^{\prime}$ be any finite map of smooth and geometrically connected curves over $K$ and suppose that $X_{K}$ and $X_{K}^{\prime}$ admit proper flat and normal models over $R$ which have rational singularities and generically smooth closed fibers. (Such models are automatically admissible due to Raynaud's critère de platitude cohomologique [Raynaud 1970, Théorème 7.2.1]). By our discussion above, $g$ induces maps $g^{*}$ and $g_{*}$ on the canonical integral structure (5-8) via pullback and trace, and induces maps $\operatorname{Alb}(g)^{*}$ and $\operatorname{Pic}^{0}(g)^{*}$ on the canonical integral structure (5-1) by Albanese and Picard functoriality via the Néron mapping property. The $R$-isomorphism (5-9) necessarily intertwines $\operatorname{Alb}(g)^{*}$ with $g^{*}$ and $\operatorname{Pic}^{0}(g)^{*}$ with $g_{*}$ as such agreement of maps between free $R$-modules may be checked after the flat scalar extension $R \rightarrow K$, where it follows from Proposition 5.4.

Question 5.8. As an interesting consequence of Corollary 5.6, the duality statement of Proposition 5.4, and the fact that the integral structure (5-8) is autodual with respect to cup-product pairing, we deduce that the autoduality of the Hodge 
filtration of $H_{\mathrm{dR}}^{1}\left(J_{K} / K\right)$ preserves the integral structure (5-1). It seems natural to ask if this is true in greater generality, that is, if for any abelian variety $A_{K}$ over $K$, the natural duality isomorphism

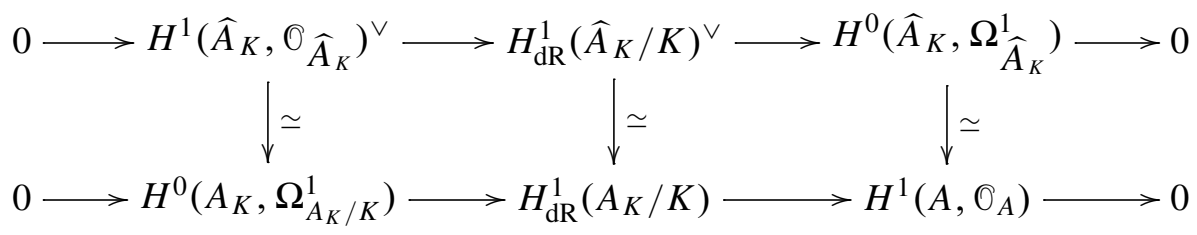

(see [Berthelot et al. 1982, Lemme 5.1.4 and Théorème 5.1.6]) identifies the corresponding canonical integral structures provided by (5-1). It is also natural to wonder how such an identification might come about at the level of canonical extensions and Néron models or more precisely if the definition of the duality for the de Rham cohomology of an abelian scheme (or more generally a 1-motive) in terms of its universal extension (see [Coleman 1991, page 636] for the case of abelian schemes, [Bertapelle 2008] for 1-motives and [Deligne 1974, 10.2.7.2] for abelian varieties over $\mathbf{C}$ ) can be extended to the case of Néron models and their canonical extensions.

Proof of Corollary 5.6. By Theorem 1.2, we have an isomorphism of short exact sequences of smooth groups as in (1-3). Applying the functor Lie and using the fact that for any group functor $G$ over $S$ with representable fibers, the inclusion $G^{0} \hookrightarrow G$ of the identity component induces an isomorphism on Lie algebras [Liu et al. 2004, Proposition 1.1 (d)], we deduce a canonical isomorphism of finite free $R$-modules

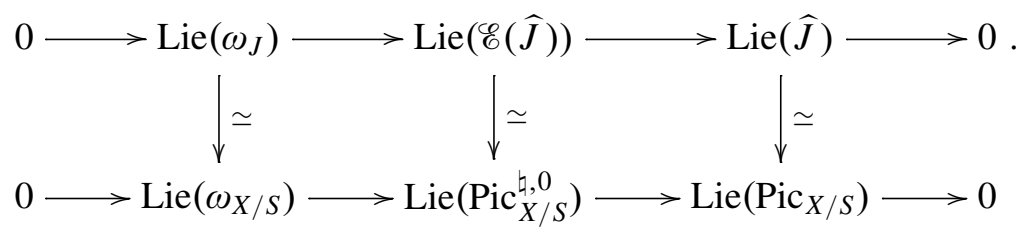

By Definition 3.8 we have the canonical identifications

$$
\begin{aligned}
\operatorname{Lie}\left(\operatorname{Pic}_{X / S}^{\natural, 0}\right) & =\operatorname{Lie}\left(\operatorname{Pic}_{X / S}^{\natural} \times \operatorname{Pic}_{X / S} \operatorname{Pic}_{X / S}^{0}\right) \\
& =\operatorname{Lie}\left(\operatorname{Pic}_{X / S}^{\natural}\right) \times{ }_{\operatorname{Lie}\left(\operatorname{Pic}_{X / S}\right)} \operatorname{Lie}\left(\operatorname{Pic}_{X / S}^{0}\right)=\operatorname{Lie}\left(\operatorname{Pic}_{X / S}^{\natural}\right),
\end{aligned}
$$

so it suffices to identify the left exact sequence of Lie algebras attached to the exact sequence of fppf abelian sheaves

$$
0 \longrightarrow f_{*} \omega_{X / S} \longrightarrow \operatorname{Pic}_{X / S}^{\natural} \longrightarrow \operatorname{Pic}_{X / S},
$$

of (3-16) with the integral structure on $H\left(X_{K}\right)$ provided by (5-8). As in Section 3, let $\omega_{X_{T} / T}^{\times, \bullet}$ be the two-term complex $d_{T} \log : \mathcal{O}_{X_{T}}^{\times} \rightarrow \omega_{X_{T} / T}$ defined by $d_{T} \log (u)=$ 
$u^{-1} \cdot d_{T}(u)$, and write $\mathbf{R}^{1} f_{*} \omega_{X / S}^{\times}$and $R^{1} f_{*} O_{X}^{\times}$, respectively, for the fppf sheaves associated to the group functors on $S$-schemes

$$
T \rightsquigarrow \mathbf{H}^{1}\left(X_{T}, \omega_{X_{T} / T}^{\times, \bullet}\right) \quad \text { and } \quad T \rightsquigarrow H^{1}\left(X_{T}, \mathcal{O}_{X_{T}}^{\times}\right) .
$$

By Lemma 3.10, the exact sequence (5-10) is naturally isomorphic to the exact sequence of fppf abelian sheaves

$$
0 \longrightarrow f_{*} \omega_{X / S} \longrightarrow \mathbf{R}^{1} f_{*} \omega_{X / S}^{\times, \bullet} \longrightarrow R^{1} f_{*} \mathrm{O}_{X}^{\times}
$$

obtained by sheafifying (3-15). Thus, the proof of Corollary 5.6 is completed by:

Lemma 5.9. There is a natural isomorphism of exact sequences of free $R$-modules

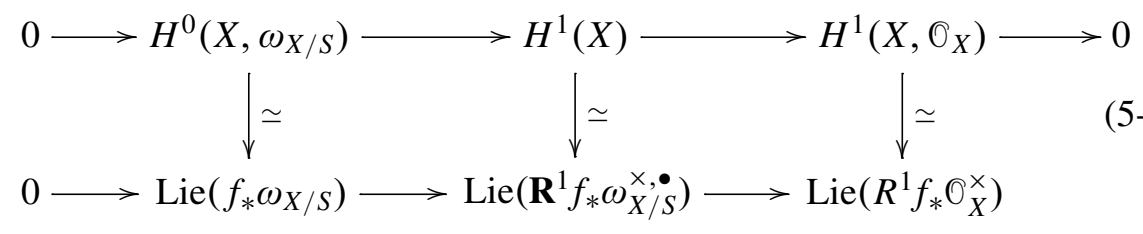

Proof. By construction, the exact sequence (5-8) results from the Hodge to de Rham spectral sequence attached to the evident filtration of $\omega_{X / S}^{\bullet}$. Now the canonical section $\mathbf{Z} \rightarrow \mathbf{Z}[\epsilon] /\left(\epsilon^{2}\right)$ to the quotient map $\epsilon \mapsto 0$ induces a canonically split exact sequence of filtered two-term (vertical) complexes

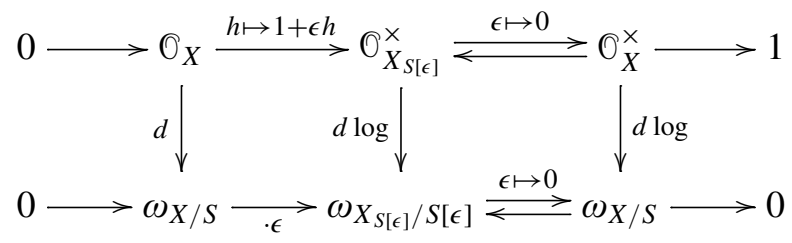

so passing to cohomology yields the commutative diagram

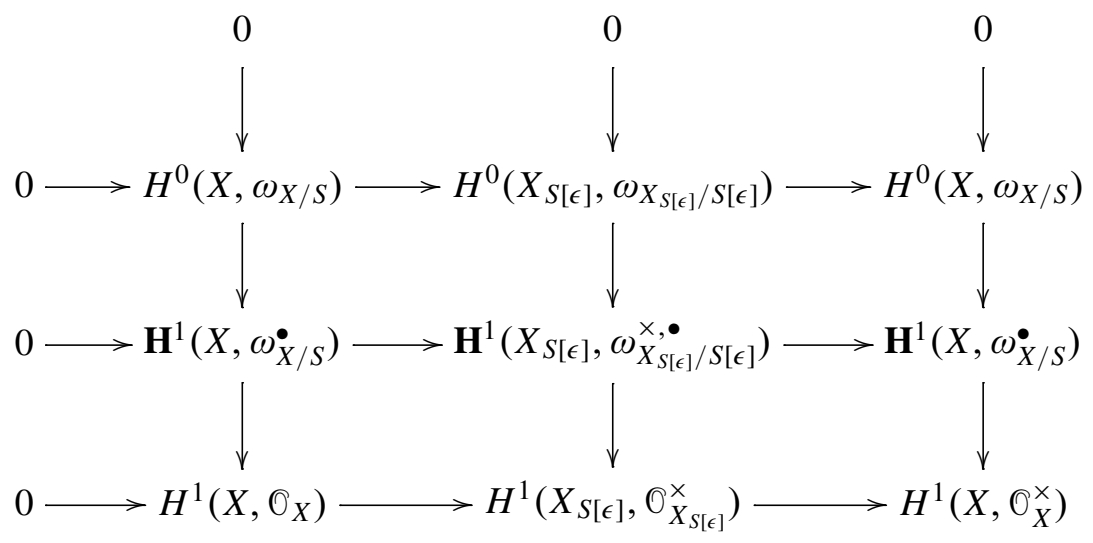

with exact rows and columns, where the zeroes in the left column result from the splitting (that is, $\mathbf{H}^{1}$ is left exact on split short exact sequences). We conclude 
that we have an isomorphism of exact sequences of abelian groups as in (5-11). It remains to show that this is in fact an $R$-linear isomorphism. Recall that for any group functor $G$ on $S$-schemes, the multiplication on $\operatorname{Lie}(G)$ by $0_{S}(S)$ is induced by the functoriality of $G$ from the map $0_{S}(S) \rightarrow \operatorname{End}_{S}(S[\epsilon])$ sending $s \in O_{S}(S)$ to the self-map $u_{s}$ of $S[\epsilon]$ that is induced by $\epsilon \mapsto s \cdot \epsilon$. Thus, the fact that the map (5-11) defined by (5-12) is a map of $R$-modules amounts to the assertion that for any $s \in \mathcal{O}_{S}(S)$ the diagram

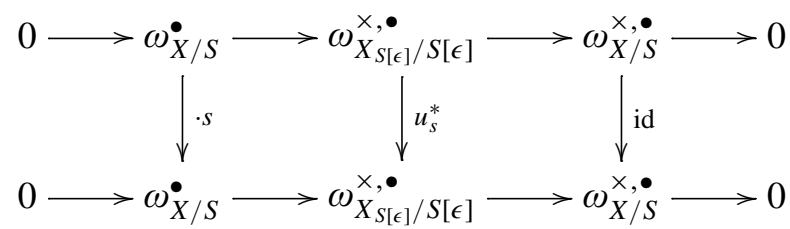

of filtered complexes with exact rows commutes. This is easily checked.

\section{Acknowledgments}

It is a pleasure to thank Dino Lorenzini, Barry Mazur, and Bill Messing for several helpful exchanges and conversations. Many thanks to Cédric Pépin, both for pointing out the argument of Proposition 2.8 to me, and for providing me with a copy of [Pépin 2008]. I would especially like to thank Brian Conrad for advising my Ph.D. thesis (which was the genesis of this article) and for many clarifications. I am very grateful to the referee for making several corrections and for suggesting a number of improvements.

\section{References}

[Bégueri 1980] L. Bégueri, Dualité sur un corps local à corps résiduel algébriquement clos, Mém. Soc. Math. France (N.S.) 4, 1980. MR 82k:12019 Zbl 0502.14016

[Bertapelle 2008] A. Bertapelle, "Deligne's duality for de Rham realizations of 1-motives", preprint, 2008. arXiv 0506344v3

[Bertapelle and Bosch 2000] A. Bertapelle and S. Bosch, "Weil restriction and Grothendieck's duality conjecture”, J. Algebraic Geom. 9:1 (2000), 155-164. MR 2000i:14065 Zbl 0978.14044

[Berthelot et al. 1982] P. Berthelot, L. Breen, and W. Messing, Théorie de Dieudonné cristalline, II, Lecture Notes in Mathematics 930, Springer, Berlin, 1982. MR 85k:14023 Zbl 0516.14015

[Bosch 1997] S. Bosch, "Component groups of abelian varieties and Grothendieck's duality conjecture”, Ann. Inst. Fourier (Grenoble) 47:5 (1997), 1257-1287. MR 98k:14061 Zbl 0919.14026

[Bosch and Lorenzini 2002] S. Bosch and D. Lorenzini, "Grothendieck's pairing on component groups of Jacobians”, Invent. Math. 148:2 (2002), 353-396. MR 2003b:14053 Zbl 1061.14042

[Bosch et al. 1990] S. Bosch, W. Lütkebohmert, and M. Raynaud, Néron models, Ergebnisse der Mathematik. (3) 21, Springer, Berlin, 1990. MR 91i:14034

[Cais 2009] B. Cais, "Canonical integral structures on the de Rham cohomology of curves", Ann. Inst. Fourier (Grenoble) 59:6 (2009), 2255-2300. 
[Coleman 1984] R. F. Coleman, "Hodge-Tate periods and p-adic abelian integrals", Invent. Math. 78:3 (1984), 351-379. MR 87f:11040 Zbl 0572.14024

[Coleman 1990] R. F. Coleman, "Vectorial extensions of Jacobians", Ann. Inst. Fourier (Grenoble) 40:4 (1990), 769-783. MR 92e:14042 Zbl 0739.14016

[Coleman 1991] R. F. Coleman, “The universal vectorial bi-extension and p-adic heights", Invent. Math. 103:3 (1991), 631-650. MR 92k:14021 Zbl 0763.14009

[Coleman 1998] R. F. Coleman, "Duality for the de Rham cohomology of an abelian scheme", Ann. Inst. Fourier (Grenoble) 48:5 (1998), 1379-1393. MR 2000j:14032 Zbl 0926.14008

[Conrad 2000] B. Conrad, Grothendieck duality and base change, Lecture Notes in Math. 1750, Springer, Berlin, 2000. MR 2002d:14025 Zbl 0992.14001

[Conrad et al. 2003] B. Conrad, B. Edixhoven, and W. Stein, “ $J_{1}(p)$ has connected fibers", Doc. Math. 8 (2003), 331-408. MR 2004k:11094 Zbl 1101.14311

[Deligne 1974] P. Deligne, “Théorie de Hodge, III”, Inst. Hautes Études Sci. Publ. Math. 44 (1974), 5-77. MR 58 \#16653b Zbl 0237.14003

[Deligne and Mumford 1969] P. Deligne and D. Mumford, "The irreducibility of the space of curves of given genus", Publ. Math. Inst. Hautes Études Sci. Publ. Math. 36 (1969), 75-109. MR 41 \#6850 Zbl 0181.48803

[EGA III 1961] A. Grothendieck and J. Dieudonné, "Éléments de géométrie algébrique, III: Étude cohomologique des faisceaux cohŕents (première partie)", Inst. Hautes Études Sci. Publ. Math. 11 (1961). MR 36 \#177c Zbl 0118.36206

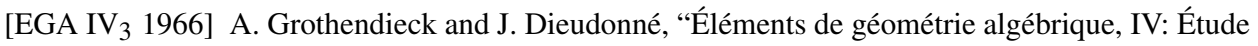
locale des schemas et des morphismes de schemas (troisième partie).", Publ. Math. Inst. Hautes Études Sci. Publ. Math. 28 (1966). MR 36 \#178 Zbl 0144.19904

[Gross 1990] B. H. Gross, "A tameness criterion for Galois representations associated to modular forms $(\bmod p)$ ", Duke Math. J. 61:2 (1990), 445-517. MR 91i:11060 Zbl 0743.11030

[Grothendieck 1974] A. Grothendieck, Groupes de Barsotti-Tate et cristaux de Dieudonné, Séminaire de Mathématiques Supérieures 45, Les Presses de l'Université de Montréal, 1974. MR 54 \#5250 Zbl 0331.14021

[Hartshorne 1966] R. Hartshorne, Residues and duality, Lecture notes in Math. 20, Springer, Berlin, 1966. MR 36 \#5145 Zbl 0212.26101

[Katz and Mazur 1985] N. M. Katz and B. Mazur, Arithmetic moduli of elliptic curves, Annals of Mathematics Studies 108, Princeton University Press, Princeton, NJ, 1985. MR 86i:11024 Zbl 0576.14026

[Lipman 1978] J. Lipman, "Desingularization of two-dimensional schemes”, Ann. Math. (2) 107:1 (1978), 151-207. MR 58 \#10924 Zbl 0349.14004

[Liu 2002] Q. Liu, Algebraic geometry and arithmetic curves, Oxford Grad. Texts in Math. 6, Oxford University Press, Oxford, 2002. MR 2003g:14001 Zbl 0996.14005

[Liu et al. 2004] Q. Liu, D. Lorenzini, and M. Raynaud, "Néron models, Lie algebras, and reduction of curves of genus one", Invent. Math. 157:3 (2004), 455-518. MR 2005m:14039 Zbl 1060.14037

[Matsumura 1989] H. Matsumura, Commutative ring theory, 2nd ed., Cambridge Stud. Adv. Math. 8, Cambridge University Press, Cambridge, 1989. MR 90i:13001 Zbl 0666.13002

[Mazur and Messing 1974] B. Mazur and W. Messing, Universal extensions and one dimensional crystalline cohomology, Lecture Notes in Math. 370, Springer, Berlin, 1974. MR 51 \#10350 Zbl 0301.14016 
[Mazur and Tate 1983] B. Mazur and J. Tate, "Canonical height pairings via biextensions", pp. 195237 in Arithmetic and geometry, vol. 1, edited by M. Artin and J. Tate, Progr. Math. 35, Birkhäuser, Boston, 1983. MR 85j:14081 Zbl 0574.14036

[McCallum 1986] W. G. McCallum, "Duality theorems for Néron models", Duke Math. J. 53:4 (1986), 1093-1124. MR 88c:14062 Zbl 0623.14023

[Messing 1972] W. Messing, The crystals associated to Barsotti-Tate groups: with applications to abelian schemes, Lecture Notes in Math. 264, Springer, Berlin, 1972. MR 50 \#337 Zbl 0243.14013

[Messing 1973] W. Messing, "The universal extension of an abelian variety by a vector group", pp. 359-372 in Symposia Mathematica, XI. Convegno di Geometria, INDAM (Rome, 1972), Academic Press, London, 1973. MR 49 \#2760 Zbl 0285.14009

[Milne 1986a] J. S. Milne, Arithmetic duality theorems, Perspectives in Math. 1, Academic Press, Boston, 1986. MR 88e:14028 Zbl 0613.14019

[Milne 1986b] J. S. Milne, "Jacobian varieties", pp. 167-212 in Arithmetic geometry (Storrs, CT, 1984), edited by G. Cornell and J. H. Silverman, Springer, New York, 1986. MR 861976 Zbl 0604.14018

[Mumford et al. 1994] D. Mumford, J. Fogarty, and F. Kirwan, Geometric invariant theory, 3rd ed., Ergebnisse der Math. (2) 34, Springer, Berlin, 1994. MR 95m:14012 Zbl 0797.14004

[Murre 1962] J. P. Murre, “On generalized Picard varieties”, Math. Ann. 145 (1962), 334-353. MR 27 \#2507 Zbl 0123.13902

[Oort 1966] F. Oort, Commutative group schemes, Lecture Notes in Math. 15, Springer, Berlin, 1966. MR 35 \#4229 Zbl 0216.05603

[Pépin 2008] C. Pépin, 2008. Private communication. To appear in author's Ph.D. thesis.

[Raynaud 1970] M. Raynaud, "Spécialisation du foncteur de Picard", Inst. Hautes Études Sci. Publ. Math. 38 (1970), 27-76. MR 44 \#227 Zbl 0207.51602

[Rosenlicht 1958] M. Rosenlicht, "Extensions of vector groups by abelian varieties", Amer. J. Math. 80 (1958), 685-714. MR 20 \#5780 Zbl 0091.33303

[SGA2 1968] A. Grothendieck, Cohomologie locale des faisceaux cohérents et théorèmes de Lefschetz locaux et globaux, Advanced Studies in Pure Mathematics 2, Masson, Paris, and NorthHolland, Amsterdam, 1968. MR 57 \#16294 Zbl 0197.47202

[SGA3-1 1970] A. Grothendieck, Schémas en groupes, I: Propriétés générales des schémas en groupes, Lecture Notes in Math. 151, Springer, Berlin, 1970. MR 43 \#223a Zbl 0207.51401

[SGA7-1 1972] A. Grothendieck, Groupes de monodromie en géométrie algébrique, I, Lecture Notes in Math. 288, Springer, Berlin, 1972. MR 50 \#7134 Zbl 0237.00013

[Tate 1958] J. Tate, "WC-groups over p-adic fields", in Séminaire Bourbaki (Exposé 156), 1958. Reprinted as pp. 265-277 in Séminaire Bourbaki 4, Soc. Math. France, Paris, 1995. MR 1610926 Zbl 0091.33701

[Wintenberger 1994] J.-P. Wintenberger, "Théorème de comparaison $p$-adique pour les schémas abéliens, I: Construction de l'accouplement de périodes”, pp. 349-397 in Périodes p-adiques (Bures-sur-Yvette, 1988), Astérisque 223, 1994. MR 96d:14019

Communicated by Brian Conrad

Received 2008-10-28

Revised 2009-07-18 Accepted 2009-08-15

bcais@math.mcgill.ca

Department of Mathematics and Statistics, McGill University, 805 Sherbrooke St. West, Montréal, QC H3A 2K6, Canada http://www.math.mcgill.ca/bcais/ 


\title{
Period, index and potential, III
}

\author{
Pete L. Clark and Shahed Sharif
}

\begin{abstract}
We present three results on the period-index problem for genus-one curves over global fields. Our first result implies that for every pair of positive integers $(P, I)$ such that $I$ is divisible by $P$ and divides $P^{2}$, there exists a number field $K$ and a genus-one curve $C_{/ K}$ with period $P$ and index $I$. Second, let $E_{/ K}$ be any elliptic curve over a global field $K$, and let $P>1$ be any integer indivisible by the characteristic of $K$. We construct infinitely many genus-one curves $C_{/ K}$ with period $P$, index $P^{2}$, and Jacobian $E$. Our third result, on the structure of ShafarevichTate groups under field extension, follows as a corollary. Our main tools are Lichtenbaum-Tate duality and the functorial properties of O'Neil's period-index obstruction map under change of period.
\end{abstract}

\section{Introduction}

1.1. Notation and conventions. Throughout the paper $K$ shall denote a global field — that is, a finite field extension of either $\mathbb{Q}$ or $\mathbb{F}_{p}(T)$ - and $E$ shall denote an elliptic curve defined over $K$.

Let $P$ be a positive integer which is not divisible by the characteristic of $K$. Define $P^{*}$ to be $P$ if $P$ is odd and $2 P$ if $P$ is even.

Let $\bar{K}$ denote a fixed separable closure of $K$, and let $\mathfrak{g}_{K}=\operatorname{Aut}(\bar{K} / K)$ be the absolute Galois group of $K$.

We abbreviate the Galois cohomology group $H^{1}\left(\mathfrak{g}_{K}, E(\bar{K})\right)$ to $H^{1}(K, E)$ and call it the Weil-Chatelet group of $E$ over $K$. Recall that this is a torsion abelian group.

Let $\operatorname{Pic}(C)$ be the Albanese/Picard variety of $C$, and $\mathbf{P i c}^{d}(C)$ the connected component classifying degree $d$ invertible sheaves on $C$, so that $\operatorname{Pic}^{0}(C)$ is the Jacobian. The letter $\eta$ shall denote an element of $H^{1}(K, E)$. Such classes $\eta$ are in canonical bijection with the set of equivalence classes of pairs $(C, \imath)$, where $C_{/ K}$ is a genus one curve, $l: \mathbf{P i c}^{0}(C) \rightarrow E$ is an isomorphism from the Jacobian of $C$ to $E$, and the equivalence is isomorphism over $K$. In other words, $l$ endows $C$ with

MSC2000: 11G05.

Keywords: period, index, Tate-Shafarevich group.

Clark is partially supported by the National Science Foundation grant DMS-0701771. 
the structure of a principal homogeneous space (or torsor) under $E$. It follows that $C_{/ K}$ itself determines, and is determined by, an orbit of $\operatorname{Aut}(E)$ on $H^{1}(K, E)$.

The period of $\eta \in H^{1}(K, E)$ is its order in the group. In terms of the corresponding torsor $C$, the period is the least positive degree of a $K$-rational divisor class on $C$. The index of $\eta$ is the gcd over all degrees $[L: K]$ of field extensions $L / K$ such that the restriction of $\eta$ to $H^{1}(L, E)$ is trivial. In terms of $C$, the index is the least positive degree of a $K$-rational divisor. By Riemann-Roch, it is also the least degree of an extension $L / K$ such that $C$ has an $L$-rational point. Since the period and index are invariant under isomorphism over $K$, we will refer to the period and index of the cohomology class $\eta$ and that of the curve $C$ interchangeably.

We denote by $\Sigma_{K}$ the set of all places of $K$ (including Archimedean places in the number field case). For a place $v$ of $K$, we denote the image of a class $\eta \in H^{1}(K, E)$ under the local restriction map $H^{1}(K, E) \rightarrow H^{1}\left(K_{v}, E\right)$ by $\eta_{v}$. In geometric terms, $\eta_{v}$ is just the base extension of the curve (or rather, the principal homogeneous space) $C$ from $K$ to $K_{v}$. By the support of a class we mean the finite set of $v \in \Sigma_{K}$ such that $\eta_{v} \neq 0$. The classes $\eta$ with empty support form a subgroup $\amalg(K, E)$, the Shafarevich-Tate group of $E_{/ K}$.

1.2. Statement of the main results. Recall that $K$ is a global field, $P$ is a positive integer not divisible by the characteristic of $K$, and $P^{*}$ is $P$ if $P$ is odd, and $2 P$ if $P$ is even.

Theorem 1. Let $E_{/ K}$ be an elliptic curve. Suppose $\# E(K)\left[P^{*}\right]=\left(P^{*}\right)^{2}$. Then, for any positive integer $D \mid P$, there are infinitely many classes $\eta \in H^{1}(K, E)$ of period $P$ and index $P \cdot D$. These classes can be chosen so as to be locally trivial except possibly at two places of $K$.

Theorem 2. Let $E_{/ K}$ be an elliptic curve and $S_{K} \subset \Sigma_{K}$ a finite set of places of $K$. There exists an infinite sequence $\left\{\eta_{i}\right\}_{i=0}^{\infty}$ of elements of $H^{1}(K, E)$ such that

- $\eta_{0}=0$

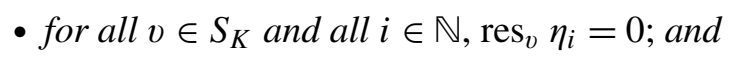

- for all $i, j \in \mathbb{N}$ with $i \neq j, \eta_{i}-\eta_{j}$ has period $P$ and index $P^{2}$.

Theorem 3. Let $E_{/ K}$ be an elliptic curve. For any positive integer $r$, there exists a degree $P$ field extension $L / K$ such that $\amalg(L, E)$ contains at least $r$ elements of order $P$.

1.3. Discussion of the results. Let $C$ be a genus-one curve over an arbitrary field $K$. It is well known (see [Lang and Tate 1958, Proposition 5], for example), that the period $P$ and the index $I$ of $C$ satisfy the divisibilities

$$
P|I| P^{2} .
$$


Conversely, Lang and Tate showed [1958, p. 678] that for any pair $(P, I)$ of positive integers satisfying (1), there exists a genus-one curve $C$ defined over the iterated Laurent series field $\mathbb{C}\left(\left(t_{1}\right)\right)\left(\left(t_{2}\right)\right)$ with period $P$ and index $I$.

This raises the question of the possible values of $P$ and $I$ for genus-one curves over a local or global field. Lichtenbaum [1968] showed that $P=I$ for every genus-one curve over a nondiscrete, locally compact field. ${ }^{1}$

Suppose $K$ is a field which admits at least one degree- $P$ cyclic extension and such that there exists an elliptic curve $E_{/ K}$ with full $P$-torsion: $\# E[P](K)=P^{2}$. Then Lang and Tate showed that there exists a class $\eta \in H^{1}(K, E)$ with period and index both equal to $P$.

Let us assume henceforth that $K$ is a global field. In this case, the argument of Lang and Tate readily yields the fact that $\eta$ may be taken to have support at most one place of $K$.

Conversely, Cassels [1962, Theorem 1.3] showed that $I=P$ for classes with empty support. Moreover $I=P$ for classes whose support has cardinality one, as was first shown by Olson [1970, Theorem 15] and "rediscovered" by the first author [Clark 2006b, Proposition 6].

The first examples of genus-one curves over a global field with $I>P$ are due to Cassels [1963], who found examples over $K=\mathbb{Q}$ with $P=2, I=4$. Cassels' examples are closely related to the theory of explicit 2-descent. More recently, the first author constructed, for any prime number $p$, classes $\eta$ with $P=p, I=p^{2}$ in the Weil-Châtelet group of any elliptic curve $E_{/ K}$ over a number field with full $p$-torsion [Clark 2005, Theorem 3]. The method crucially employs a period-index obstruction map due to O'Neil [2002].

Our Theorem 1 may therefore be viewed as a generalization of [Clark 2005, Theorem 3]. In particular, we now know that any pair $(P, I)$ satisfying $(1)$ arises as the period and index of a genus-one curve defined over some number field (depending on $P$ ). Moreover, the fact that we can construct such classes which are supported at two places is, in view of the aforementioned results of Cassels and Olson, optimal, and answers a question raised by Çiperiani.

Having established Theorem 1, we naturally wish to understand the possible values of period and index for genus-one curves defined over a fixed global field $K$, or - better yet - inside the Weil-Châtelet group $H^{1}(K, E)$ of a fixed elliptic curve $E_{/ K}$.

Our Theorem 2 shows that for any elliptic curve $E$ over a global field $K$ and any $P>1$ indivisible by the characteristic of $K$, there exist infinitely many genusone curves with period $P$, index $P^{2}$ and Jacobian $E$. Of course the statement

\footnotetext{
${ }^{1}$ More precisely, Lichtenbaum proved this under the assumption that $P$ is not divisible by the characteristic of $K$ - the same assumption which is in force for us - but Milne [1972] later extended Tate's local duality theory to this case and accordingly removed this hypothesis.
} 
of Theorem 2 is significantly more complicated than this, and its significance is probably hard to appreciate. However, we need this precise statement, especially the "difference properties" of the sequence $\left\{\eta_{i}\right\}$, in the proof of Theorem 3.

In order to place Theorem 3 into context, let us again recall some prior results, this time on the problem of constructing "large Shafarevich-Tate groups." More precisely, we fix a global field $K$, an integer $P>1$ and a positive integer $r$, and the goal is prove the existence of an elliptic curve $E_{/ K}$ whose Shafarevich-Tate group $\amalg(K, E)$ contains at least $r$ elements of order $P$.

The first results here are due to Cassels [1964], who solved the aforementioned problem for $K=\mathbb{Q}$ and $P=3$. (This was also the first proof of the weaker fact that $\amalg(\mathbb{Q}, E)$ is unbounded as $E$ ranges over all elliptic curves $E_{/ \mathbb{Q} .}$.) Cassels' examples all have $j=0$ and exploit the extra structure on such curves afforded by the existence of an order 3 automorphism. The problem has also been solved for $P=2$ by Bölling [1975], and for $P=5$ by Fisher [2001]. Donnelly [2003] established the result for $P=7$. Further, the case $P=13$ is proved separately by Donnelly (unpublished) and Matsuno [2007]. Among prime values of $P$, this is a transitional case: the modular curve $X_{0}(P)$ has genus 0 precisely for these values of $P$. There are as yet no such results for larger $P$.

There has also been work showing that, for a prime $p$, either the $p$-Selmer group $\operatorname{Sel}^{p}(K, E)$ or $\amalg(K, E)[p]$ can be made arbitrarily large when one varies over all elliptic curves $E$ defined over number fields $K$ whose degree $[K: \mathbb{Q}]$ is bounded by a certain function of $p$. Notably, Kloosterman and Schaefer [2003] showed that $\operatorname{dim}_{\mathbb{F}_{p}} \operatorname{Sel}^{p}(K, E)$ is unbounded as $K$ ranges over all field extensions $K / \mathbb{Q}$ of degree $f_{1}(p)=O(p)$. Kloosterman [2005] showed that $\operatorname{dim}_{\mathbb{F}_{p}} \amalg(K, E)[p]$ is unbounded as $K$ ranges over extensions of degree $f_{2}(p)=O\left(p^{4}\right)$.

In [Clark 2005, Theorem 1], it was shown that if $\# E(K)[p]=p^{2}$ for a prime $p$, then $\amalg(L, E)[p]$ is unbounded as $L$ ranges over all degree $p$ field extensions. The argument can be applied to any elliptic curve defined over a global field (of characteristic not divisible by $p$ ) at the cost of first trivializing the Galois action on the $p$-torsion. It follows that for every $E_{/ K}, \amalg(L, E)[p]$ is unbounded as $L$ ranges over extensions of degree at most $f_{3}(p)=p\left(p^{2}-1\right)\left(p^{2}-p\right) \leq p^{5}$. Moreover, upon restricting to elliptic curves with potential complex multiplication, one gets the bound $f_{4}(p) \leq 2 p^{3}$.

In contrast, our Theorem 3 extends the bound $[L: K]=P$ of [Clark 2005, Theorem 1] to all elliptic curves and all integers $P>1$. An interesting question (which we are not able to answer) is whether Theorem 3 is in fact the optimal result of its kind.

1.4. Organization of the paper. We assume some familiarity with the literature on the period-index problem, especially [O'Neil 2002; Clark 2005]; nevertheless, we 
begin with a brief review of the period-index obstruction map, and then go on to discuss some new ideas and techniques. The first key point is a clarification of the relationship between O'Neil's obstruction map $\Delta$ and the quantity $I / P$. Whereas before it had been implicit in [O'Neil 2002] (and explicit in [Clark 2005]) that one can use $\Delta$ to determine whether or not $I=P$, here we present a simple characterization of $I / P$ in terms of the obstruction to a rational divisor class being represented by a rational divisor. We also return to the point of the explicit computation of O'Neil's obstruction map in the case of full-level $N$ structure for even $N$. These matters are detailed in Section 2.

In Section 3 we give the proofs of Theorems 1, 2, and 3.

\section{On the period-index obstruction map}

In this section $K$ is an arbitrary field, $E_{/ K}$ is an elliptic curve, and $P$ is a positive integer not divisible by the characteristic of $K$. These hypotheses ensure that the finite flat $K$-group scheme $E[P]$ is étale, and so may be viewed as a $\mathfrak{g}_{K}$-module.

2.1. Three aspects of the period-index obstruction map. The key technical tool in the proofs of our results is the period-index obstruction map

$$
\Delta_{P}: H^{1}(K, E[P]) \rightarrow \operatorname{Br}(K) .
$$

It can be defined in three different ways, which we now recall. All three characterizations either explicitly appear in or are readily deducible from [O'Neil 2002]. Note that $\Delta_{P}$ is not a homomorphism; as we shall see, it is a quadratic map.

Definition 1. For any ample line bundle $L$ on an abelian variety $A_{/ K}$, the functor $\mathscr{G}_{L}$ which associates to a $K$-scheme $S$ the group of all isomorphisms

$$
(x, \psi): L_{/ S} \stackrel{\sim}{\rightarrow} \tau_{x}^{*}\left(L_{/ S}\right)
$$

between $L_{/ S}$ and one of its translates is represented by a $K$-group scheme, Mumford's theta group. The subgroup of automorphisms of $L$ gives rise to an embed$\operatorname{ding} \mathbb{G}_{m} \hookrightarrow \mathscr{G}_{L}$. The quotient is canonically isomorphic to $\kappa(L)$, the kernel of the canonical homomorphism

$$
\varphi_{L}: A \rightarrow A^{\vee}, x \mapsto \tau_{x}^{*}(L) \otimes L^{-1} .
$$

We now follow O'Neil's construction [2002, §2]. Let $A$ be an elliptic curve $E$ and $L$ the line bundle associated to the divisor $P[O]$ on $E$; note that $\kappa(L)=E[P]$. Let $\varphi_{L}: E \rightarrow \mathbb{P}^{P-1}$ be the associated morphism into projective space (well-defined up to a linear automorphism of $\mathbb{P}^{P-1}$ ). 
Proposition 4. For $P \geq 2$ we have the following commutative diagram of group schemes:

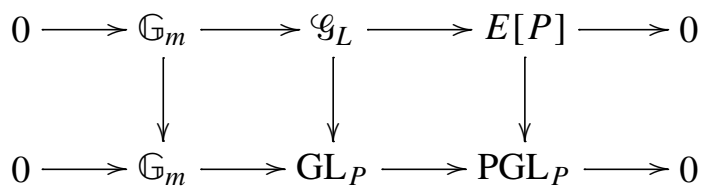

Proof. This is [O'Neil 2002, Proposition 2.1]. For our purposes, we will only need to know the vertical map on the right. We view $E[P]$ as an automorphism group of $\varphi_{L}: E \rightarrow \mathbb{P}^{P-1}$ - that is, an element of $E[P]$ acts on the global sections of $L$, and thus induces an automorphism of $\mathbb{P}^{P-1}$. This gives an element of $\mathrm{PGL}_{P}$ as required.

The machinery of nonabelian Galois cohomology [Serre 1962] supplies a connecting map from $H^{1}(K, E[P]) \rightarrow H^{2}\left(K, \mathbb{G}_{m}\right)$. Identifying $H^{2}\left(K, \mathbb{G}_{m}\right)$ with $\operatorname{Br}(K)$, we obtain our first definition of $\Delta_{P}$.

Definition 2. Let $V_{/ K}$ be any nonsingular, complete, geometrically integral variety, and let $\operatorname{Pic}(V)$ be the Picard group of $V$. There is an exact sequence [Bosch et al. $1990, \S 9.1]$

$$
0 \rightarrow \operatorname{Pic}(V) \rightarrow \operatorname{Pic}(V)(K) \stackrel{\delta_{V}}{\rightarrow} \operatorname{Br}(K) \stackrel{\gamma}{\rightarrow} \operatorname{Br}(V)
$$

In particular, given a $K$-rational divisor class $D$ on $V$, the obstruction to $V$ being represented by a $K$-rational divisor is an element of $\operatorname{Br}(K)$. A Galois descent argument shows that $H^{1}(K, E[P])$ classifies pairs $(C, D)$ - where $C \in H^{1}(K, E)$ and $D \in \operatorname{Pic}^{P}(C)(K)$ is a $K$-rational divisor class - modulo the relation $(C, D) \sim$ $\left(C^{\prime}, D^{\prime}\right)$ if there exists a $K$-isomorphism of torsors $f: C \rightarrow C^{\prime}$ with $f^{*} D^{\prime}=D$. One may then define

$$
\Delta_{P}((C, D))=\delta_{C}(D) .
$$

For details, including a proof of the equivalence of this definition with the previous one, see [O’Neil 2002, Proposition 2.3] and [Clark 2005, Proposition 4].

Definition 3. On the other hand, $H^{1}(K, E[P])$ classifies $K$-morphisms $\varphi: C \rightarrow V$ which are twisted forms of $\varphi_{L}: E \rightarrow \mathbb{P}^{P-1}$; these forms arise as twists of the map associated to the complete linear system $P[O]$. In particular, $C \in H^{1}(K, E)$ and $V$ is a twisted form of $\mathbb{P}^{P-1}$; that is, a Severi-Brauer variety [O'Neil 2002; Cremona et al. 2008, §1.2]. We may then define $\Delta_{P}(\varphi: C \rightarrow V)=[V]$, the class of $V$ in $\operatorname{Br}(K)$. It follows that $\Delta_{P}\left(H^{1}(K, E[P])\right)$ consists of elements of $\operatorname{Br}(K)$ whose index divides $P$; a fortiori we have the important relation

$$
\Delta_{P}\left(H^{1}(K, E[P])\right) \subset \operatorname{Br}(K)[P] .
$$


2.2. Lichtenbaum-Tate Duality. As above, we let $E$ be an elliptic curve defined over an arbitrary field $K$, and now let $n$ denote a positive integer indivisible by the characteristic of $K .^{2}$ We have the Kummer sequence

$$
0 \rightarrow E(K) / n E(K) \stackrel{l}{\rightarrow} H^{1}(K, E[n]) \rightarrow H^{1}(K, E)[n] \rightarrow 0 .
$$

Using $\imath$ and $\Delta$, we may define a map Li : $H^{1}(K, E[n]) \times E(K) \rightarrow \operatorname{Br}(K)$ by

$$
\operatorname{Li}(\xi, x)=\Delta(\xi+l(x))-\Delta(\xi)-\Delta(l(x)) .
$$

Since $\Delta(l(E(K) / n E(K)))=0$, Li depends only on the image of $\xi$ in $H^{1}(K, E)[n]$ and on the image of $x$ in $E(K) / n E(K)$; that is, it descends to give a map

$$
\mathrm{Li}: H^{1}(K, E)[n] \times E(K) / n E(K) \rightarrow \operatorname{Br}(K)[n] .
$$

We also have the Tate pairing

$$
T: H^{1}(K, E)[n] \times E(K) / n E(K) \rightarrow \operatorname{Br}(K)[n] .
$$

There are many definitions of the Tate pairing; see for example [Tate 1958; Lichtenbaum 1969]. Perhaps the most straightforward is as follows. Given $(\xi, x)$, lift $\xi$ to any $\eta$ in $H^{1}(K, E[n])$. Consider the cup product

$$
\eta \cup{ }_{l}(x) \in H^{2}(K, E[n] \otimes E[n]),
$$

and follow by the Weil pairing to obtain a class in $H^{2}\left(K, \mu_{n}\right)$; the latter is canonically isomorphic to $\operatorname{Br}(K)[n]$. The resulting Brauer class is $T(\xi, x)$. Note that the pairing is independent of our choice of $n$, in the sense that we may replace $n$ by any multiple without changing the value of the pairing.

Theorem 5 [O’Neil 2002, §5]. The map Li coincides with the Tate pairing T.

Since $T$ is bilinear, the theorem implies that so is $\mathrm{Li}$, and together with the fact that $\Delta(d \xi)=d^{2} \Delta(\xi)$ [O'Neil 2002, Lemma 4.2] this means that $\Delta$ itself is a quadratic map. This also follows from the first definition of $\Delta$ as a connecting map in nonabelian cohomology, together with [Zarhin 1974]. Note that if $K$ is complete, discretely valued, and has finite residue field, then $\operatorname{Br}(K)[n]=\left(\frac{1}{n} \mathbb{Z}\right) / \mathbb{Z}$, and Li puts the finite abelian groups $H^{1}(K, E)[n]$ and $E(K) / n E(K)$ in Pontrjagin duality ("Tate local duality").

\footnotetext{
${ }^{2}$ Thus $n$ satisfies exactly the same requirements as our "fixed" positive integer $P$. The merit of considering both "fixed $P$ " and "variable $n$ " will become clear in the next section.
} 
2.3. Theta functoriality. Let $\eta$ be a class in $H^{1}(K, E)[n]$. The exactness of the Kummer sequence (4) means that $\eta$ has at least one lift to an element

$$
\xi \in H^{1}(K, E[n]) .
$$

Following O'Neil and Clark, we attempt to use the obstruction maps $\Delta$ to study the discrepancy between the period and the index of $\eta$.

Now a key point: in [Clark 2005] we only considered the case where $n$ is equal to the period $P$ of $\eta$. But certainly we can also choose lifts $\xi_{n} \in H^{1}(K, E[n])$ whenever $n$ is any multiple of the period of $\eta$. It turns out to be quite useful to do so, and in particular to compare various obstruction maps $\Delta_{n}$ of differing levels. Geometrically speaking this amounts to considering along with the theta group $\varphi_{L}$ of our fixed line bundle $L=L(P[O])$ the theta groups of all tensor powers $L^{m}$ of $L$ and various natural homomorphisms between them. The study of such homomorphisms is an integral part of Mumford's theory.

So let $m$ be yet another positive integer indivisible by the characteristic of $K$. The natural inclusion $E[P] \hookrightarrow E[m P]$ of $\mathfrak{g}_{K}$-modules induces a map

$$
j_{m}: H^{1}(K, E[P]) \rightarrow H^{1}(K, E[m P]) .
$$

Under the interpretation 2 of $H^{1}(K, E[P])$ as equivalence classes of pairs $(C, D)$, where $C \in H^{1}(K, E)$ and $D \in \operatorname{Pic}^{P}(C), j_{m}$ is the map $(C, D) \mapsto(C, m D)$.

Similarly, multiplication by $m$ induces a map

$$
[m]: H^{1}(K, E[m P]) \rightarrow H^{1}(K, E[P]) .
$$

Proposition 6. If $\xi \in H^{1}(K, E[P])$ and $\eta \in H^{1}(K, E[m P])$, then:

(a) $\Delta_{m P} j_{m}(\xi)=m \Delta_{P}(\xi)$, and

(b) $m \Delta_{m P} \eta=\Delta_{P}([m] \eta)$.

Proof. Mumford [1966, pp. 309-310] shows that both $j_{m}$ and $[m]$ extend to morphisms of the theta group sequences:

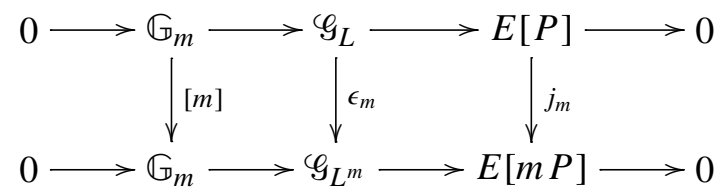

and

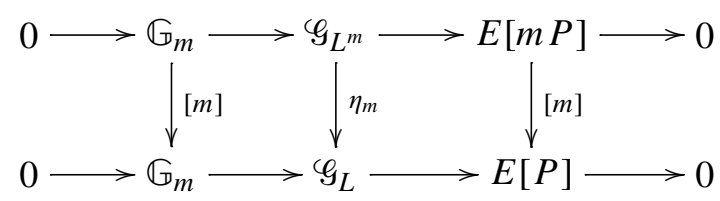


In each case the restriction to $\mathbb{G}_{m}$ is simply the $m$ th power map. We remark that the map $\epsilon_{m}: \mathscr{G}_{L} \rightarrow \mathscr{G}_{L^{m}}$ is relatively straightforward to define: an isomorphism $\psi: L \stackrel{\sim}{\rightarrow} \tau_{x}^{*} L$ induces, by passage to the $m$ th power, a canonical isomorphism $\psi^{\otimes m}: L^{m} \stackrel{\sim}{\rightarrow} \tau_{x}^{*}\left(L^{m}\right)$, so $\epsilon_{m}:(x, \psi) \mapsto\left(x, \psi^{m}\right)$. These commutative ladders induce commutative ladders in nonabelian Galois cohomology, and the commutativity of these last two diagrams gives the desired result.

2.4. Applications to the quantity $I / P$. We begin with the following result, which was known to O'Neil:

Proposition 7 [Clark 2005, Theorem 5]. Let $E_{/ K}$ be an elliptic curve over a field $K$, and $P$ a positive integer indivisible by the characteristic of $K$. Let $\eta \in$ $H^{1}(K, E)$ be of period $P$. The following are equivalent:

(a) $\eta$ has index $P$.

(b) There exists some lift $\xi \in H^{1}(K, E[P])$ of $\eta$ such that $\Delta_{P}(\xi)=0$.

Proof. If $C$ is the genus-1 curve represented by $\eta$, then in light of the second definition of $\Delta_{P}$, both conditions express the fact that $C$ admits a rational divisor of degree $P$.

We are therefore interested in the remaining case in which $\Delta_{P}(\xi) \neq 0$ for every lift $\xi$ of $\eta$ to $H^{1}(K, E[P])$.

Let $C_{/ K}$ be a curve of any genus, of period $P$ and index $I$. Referring back to (3), we may define the relative Brauer group $\kappa(C / K)=\operatorname{Im}\left(\delta_{C}\right)=\operatorname{Ker}(\gamma)$. For any $n \in \mathbb{Z}$, define moreover $\kappa^{n}(C / K)=\delta_{C}\left(\operatorname{Pic}^{n}(C)(K)\right)$.

Proposition 8. The quotient $\kappa(C / K) / \kappa^{0}(C / K)$ is cyclic of order $I / P$.

This is a reasonably well-known result [Ciperiani and Krashen 2007, Theorem 2.1.1; Clark 2006a, Proposition 24], the standard proof of which employs a snake lemma argument. But the following proof offers some additional insight.

Proof. By the definition of $P$ we have $\operatorname{Pic}^{n}(C)(K)=\varnothing$ unless $n$ is a multiple of $P$, so

$$
\begin{aligned}
\kappa(C / K)=\delta_{C}(\operatorname{Pic}(C)(K))=\delta_{C}\left(\bigcup_{n \in \mathbb{Z}} \operatorname{Pic}^{n P}(C)(K)\right) \\
\quad=\bigcup_{n \in \mathbb{Z}} \delta_{C}\left(\operatorname{Pic}^{n P}(C)(K)\right)=\bigcup_{n \in \mathbb{Z}} \kappa^{n P}(C / K) .
\end{aligned}
$$

Choose a rational divisor class $D$ of degree $P$; this in turn determines a rational divisor class of each degree $n P$, namely $D_{n P}=n D$. Put $\alpha=\delta_{C}(D)$, so that $\delta_{C}\left(D_{n P}\right)=n \alpha$. Adding $D_{n P}$ induces a bijection of sets $\mathbf{P i c}^{0}(C)(K) \rightarrow \mathbf{P i c}^{n P}(C)$, and exhibits

$$
\kappa^{n P}(C / K)=n \alpha+\kappa^{0}(C / K)
$$


as a coset of the subgroup $\kappa^{0}(C / K)$ of $\operatorname{Br}(K)$. This shows that $\kappa(C / K)$ is the subgroup generated by $\alpha$ and $\kappa^{0}(C / K)$. Moreover, $C$ admits a rational divisor of degree $n P$ if and only if $0 \in \kappa^{n P}(C / K)$ if and only if $n \alpha \in \kappa^{0}(C / K)$. The quantity $I / P$ is the least such value of $n$, that is, the order of

$$
\left\langle\alpha+\kappa^{0}(C / K)\right\rangle / \kappa^{0}(C / K)=\kappa(C / K) / \kappa^{0}(C / K) .
$$

Proposition 9. Let $\eta \in H^{1}(K, E)$ be a class with period $P$ and index $I$, and let $\xi$ be any lift of $\eta$ to $H^{1}(K, E[P])$. Then

$$
I / P \leq \min _{x \in E(K) / P E(K)} \operatorname{order}\left(\Delta_{P}(\xi+\imath(x))\right) .
$$

Proof. As $x$ runs through $E(K) / P E(K)$, the elements $\xi+x$ run through all lifts of $\eta$ to $H^{1}(K, E[P])$. For any such lift $\xi$, let $D=\operatorname{order}\left(\Delta_{P}(\xi)\right)$. Then $\Delta_{P D}\left(j_{D}(\xi)\right)=$ $D \Delta_{P}(\xi)=0$, so that there is a rational divisor of degree $P D$ on the corresponding torsor, and $I \leq P D$.

Concerning the inequality (7), Proposition 7 says that the left-hand side equals 1 if and only if the right-hand side does. When $P=p$ is prime, we have a simple dichotomy: either $I / P=1$ or $I / P=p$, so equality holds in (7) when the period is prime, a fact which was exploited in [Clark 2005]. By a primary decomposition argument, we also have equality when $P$ is square-free. It is not hard to see that equality holding in (7) is equivalent to the splitting of the short exact sequence

$$
0 \rightarrow \kappa^{0}(C / K) \rightarrow \kappa(C / K) \rightarrow Q \rightarrow 0,
$$

where the last term $Q$ is cyclic of order $I / P$. It is natural to wonder whether this sequence always splits. This innocuous-looking question lies at the heart of the relationship between the period, the index and the period-index obstruction map, and it turns out to be surprisingly difficult. We believe that the answer is in general negative. However it is possible to show that equality holds for certain specially constructed classes. In the proofs of the main theorems we use Lichtenbaum-Tate duality to ensure equality, following [Sharif 2006].

2.5. The case of full-level P structure. In this section we assume that $E[P](\bar{K}) \subset$ $E(K)$. By the theory of the Weil pairing, the $P$ th roots of unity $\mu_{P}$ are contained in $K$. Fix a basis $(S, T)$ for $E[P]$ once and for all. Note that this induces, via the Weil pairing, a basis for $\mu_{P}$ - that is, a specific primitive $P$ th root of unity $\zeta=e_{P}(S, T)$. After making this choice, we get an isomorphism

$$
\Phi: H^{1}\left(K, \mu_{P}\right) \times H^{1}\left(K, \mu_{P}\right) \stackrel{\sim}{\rightarrow} H^{1}(K, E[P]) .
$$

The composition of the cup product with the map $\mu_{P} \otimes \mu_{P} \rightarrow \mu_{P}$ given by

$$
\zeta^{a} \otimes \zeta^{b} \mapsto \zeta^{a b}
$$


gives a pairing

$$
\langle,\rangle_{P}: H^{1}\left(K, \mu_{P}\right) \times H^{1}\left(K, \mu_{P}\right) \rightarrow H^{2}\left(K, \mu_{P}\right)=\operatorname{Br}(K)[P],
$$

the level P norm-residue symbol (or Hilbert symbol) [Serre 1962, p. 207].

Via the canonical Kummer isomorphism $H^{1}\left(K, \mu_{P}\right)=K^{\times} / K^{\times P}$, we may equally well view $\Phi$ and $\langle,\rangle_{P}$ as maps defined on $\left(K^{\times} / K^{\times P}\right)^{2}$.

Theorem 10. If $E\left[P^{*}\right] \subset E(K)$, then $\Delta_{P} \circ \Phi=\langle,\rangle_{P}$.

As a prelude to the proof, we consider the special theta group. Recall the theta group scheme $\mathscr{G}_{L}$, where $L$ is the class of $P[O]$. We found a homomorphism from $\mathscr{G}_{L}$ to $\mathrm{GL}_{P}$. Form the fiber product

$$
\mathscr{S}_{L}=\mathscr{G}_{L} \times_{\mathrm{GL}} \mathrm{SL}_{P}
$$

where $\mathrm{SL}_{P} \subset \mathrm{GL}_{P}$ is the special linear group. Then we have an exact sequence

$$
0 \rightarrow \mu_{P} \rightarrow \mathscr{S}_{L} \rightarrow E[P] \rightarrow 0
$$

where the maps are the restrictions of the maps in (2). If we identify $H^{2}\left(K, \mu_{P}\right)$ with $(\mathrm{Br} K)[P]$, then the coboundary $H^{1}(K, E[P]) \rightarrow H^{2}\left(K, \mu_{P}\right)$ is the obstruction map. Let $c: H^{0}(K, E[P]) \rightarrow H^{1}\left(K, \mu_{P}\right)$ be the lower dimension coboundary. Define

$$
d: H^{1}(K, E[P]) \rightarrow(\operatorname{Br} K)[P]
$$

to be given by $d \xi(\sigma, \tau)=c(\xi(\tau))(\sigma)$. (Note that since $E[P]$ is a trivial Galois module, each cohomology class in $H^{1}(K, E[P])$ consists of a single cocycle.)

Lemma 11. $\Delta=\langle,\rangle \circ \Phi^{-1}+d$.

Proof. As mentioned above, we have earlier shown [Clark 2005, Theorem 6] that

$$
\Delta-\langle,\rangle \circ \Phi^{-1}
$$

is a homomorphism of groups. Therefore it suffices to prove the claim for any subset of $H^{1}(K, E[P])$ which generates the group. We will consider the subset given by the images of $H^{1}(K, \mathbb{Z} / P \mathbb{Z})$ induced by the two maps $(1 \mapsto S)$ and $(1 \mapsto T)$. By symmetry, it suffices to consider the case $(1 \mapsto S)$ only. Let $a \in$ $\operatorname{Hom}\left(\mathfrak{g}_{K}, \mathbb{Z} / P \mathbb{Z}\right)$, and let $\xi$ be the image of $a$ under the map $(1 \mapsto S)$. Clearly $\left\langle\Phi^{-1}(\xi)\right\rangle=0$. Map $S$ down to $\operatorname{PGL}_{P}(K)$, then lift to an element $M_{S}$ in $\operatorname{SL}_{P}(\bar{K})$. We set $M_{a S}=M_{S}^{a}$. Note that since $\operatorname{det} M_{S}=1$ and $S$ has order $P$, we must have 
$M_{S}^{P}=I$. Then

$$
\begin{aligned}
(\Delta \xi)(\sigma, \tau) & =M_{S}^{a(\sigma)}\left(\sigma M_{S}^{a(\tau)}\right) M_{S}^{-a(\sigma \tau)} \\
& =M_{S}^{a(\sigma)} a(\tau) \cdot c(S)(\sigma) M_{S}^{a(\tau)} M_{S}^{-a(\sigma \tau)} \\
& =a(\tau) \cdot c(S)(\sigma) \\
& =c(\xi(\tau))(\sigma) \\
& =d \xi(\sigma, \tau) .
\end{aligned}
$$

The second equality follows from the fact that $c(S)(\sigma)=\left(\sigma M_{S}\right) M_{S}^{-1}$.

Lemma 12. $2 d=0$.

Proof. It suffices to show that $2 c=0$. Let $\imath$ be the group inverse map on $E[P]$. According to [Mumford 1966, p. 308], $\imath$ extends to a map on the theta group $\mathscr{G}_{L}$ which acts as the identity on $\mathbb{G}_{m}$. We restrict $l$ to $\mathscr{S}_{L}$. By the functoriality of $c$, if $x \in H^{0}(K, E[P])=E[P]$, then $c \circ l(x)=c(x)$. But $c \circ l(x)=c(-x)=-c(x)$, which proves the claim.

Proof of Theorem 10. If $P$ is odd, then $H^{1}\left(K, \mu_{P}\right)$ has trivial 2-torsion. Therefore Lemma 12 implies that $d=0$. By Lemma 11, the conclusion follows.

Now suppose $P$ is even. According to [Mumford 1966, p. 310], there is a map $\eta_{2}: \mathscr{G}_{L^{2}} \rightarrow \mathscr{G}_{L}$ which, upon restriction to the subgroup schemes $\mathscr{S}_{L}$ and $\mathscr{I}_{L^{2}}$, induces the commutative diagram

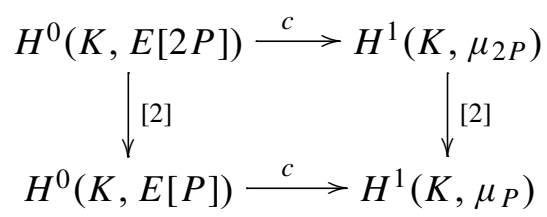

By the proof of Lemma 12, [2] $\circ c$ is the zero map. Therefore $c \circ[2]$ is zero. The hypothesis $E[2 P] \subset E(K)$ implies that the left-hand map above is surjective, and therefore the lower map $c$ is zero. By Lemma 11, the result follows.

\section{Proofs of Theorems 1,2 and 3}

We first remind the reader of a standard trick: in all work on the period-index problem it suffices to treat the case where the period $P$ is a prime power $P=p^{a}$. Indeed, if a class $\eta \in H^{1}(K, E)$ (or any other Galois cohomology group, for that matter) has period $P=p_{1}^{a_{1}} \ldots p_{r}^{a_{r}}$, then putting $\eta_{i}=\left(P / p_{i}^{a_{i}}\right) \eta$, one easily checks that $\eta=\sum_{i=1}^{r} \eta_{i}$ and that $I(\eta)=\prod_{i=1}^{r} I\left(\eta_{i}\right)$ (that is, the index of $\eta$ is the product of the indices of the classes $\eta_{i}$ ). The advantage of reducing to the case $P=p^{a}$ is that then the index $I=p^{b}$ for $a \leq b \leq 2 a$ and then for any $D=p^{c}$, if the index $I$ is less than $D P$, then indeed $I$ is a proper divisor of $D P$. 
3.1. Conditions on prime ideals and their generators. Several times in the proofs we will be choosing pairs of prime ideals $v, v^{\prime}$ of $O_{K}$ so as to satisfy certain conditions. Let us first say that a prime ideal $v$ of $K$ is "bad" (for $E$ and $P=p^{a}$ ) if $v$ is Archimedean, $v$ divides $p$, or $E$ has bad reduction at $v$, and is "good" otherwise. All but finitely many primes are good.

The conditions we will impose on $v$ and $v^{\prime}$ can all be achieved by using the Chebotarev density theorem. The conditions are:

(SC1) The primes $v=(\pi)$ and $v^{\prime}=\left(\pi^{\prime}\right)$ are principal, with totally positive generators $\pi$ and $\pi^{\prime}$.

(SC2) All elements of $E(K)$ are $P$-divisible in $E\left(K_{v}\right)$.

(SC3) The generators $\pi$ and $\pi^{\prime}$ lie in $K_{w}^{\times P}$ for all bad primes $w$.

(SC4) The order of the image of $\pi^{\prime}$ in $K_{v}^{\times} / K_{v}^{\times P}$ is $P$.

Lemma 13. Suppose that $E[P] \subset E(K)$. Then there exist infinitely many pairs of primes $v=(\pi)$ and $v^{\prime}=\left(\pi^{\prime}\right)$ satisfying conditions $(\mathrm{SC} 1)-(\mathrm{SC} 4)$.

Proof. In order to satisfy condition (SC4), we will need to choose $v$ first, as $v^{\prime}$ depends on this choice. However, the procedure for choosing the two is similar, so the argument below is presented for both at once.

Condition (SC1) is equivalent to $v$ and $v^{\prime}$ splitting completely in the Hilbert class field of $K$, while condition (SC2) is equivalent to $v$ splitting completely in $K\left([P]^{-1} E(K)\right)$, the field obtained by adjoining to $K$ all points $Q \in E(\bar{K})$ such that $[P] Q \in E(K)$. (Recall that under the hypothesis $E[P] \subset E(K), K\left([P]^{-1} E(K)\right)$ is a finite abelian extension of $K$ unramified outside the bad primes [Silverman 1986, p. 194].)

Let $\mathfrak{m}$ be the modulus given by the product of all bad primes $\mathfrak{p}$ and $P^{2}$. Then one can find $\pi$ and $\pi^{\prime}$ as in (SC3) provided $v$ and $v^{\prime}$ split completely in the ray class field for $K$ modulo $\mathfrak{m}$. For if $v$ splits completely, it has trivial Frobenius and, by class field theory, has a generator $\pi$ which is congruent to $1(\bmod \mathfrak{m})$. The condition follows from Hensel's Lemma.

Therefore, to satisfy conditions (SC1)-(SC3), we need $v$ and $v^{\prime}$ to split completely in the abelian extension $F$ which is the compositum of the Hilbert class field of $K, K\left([P]^{-1} E(K)\right)$, and the ray class field $K_{\mathfrak{m}}$.

Now we consider (SC4). Suppose that we have chosen $v$ already. Let $\alpha$ be a unit in $K_{v}$ which has order $P$ in $K_{v}^{\times} / K_{v}^{\times P}$. Let $F^{\prime}$ be the ray class field with modulus $v$. By class field theory, the Galois group of $F^{\prime} / K$ is isomorphic to the ideal class group with modulus $v, C_{v}$. In particular, if $v^{\prime}$ and $(\alpha)$ lie in the same class in $C_{v}$, then $v^{\prime}$ has a generator $\pi^{\prime}$ which is congruent to $\alpha(\bmod v)$, and hence satisfies (SC4). 
Thus, we have reduced conditions (SC1)-(SC4) to two splitting-type conditions in the abelian extensions $F$ and $F^{\prime}$. It suffices to show that these splitting conditions are compatible, since then the Chebotarev density theorem shows there are infinitely many primes satisfying the conditions.

The extension $F / K$ is unramified at $v$, while $F^{\prime} / K$ is unramified outside $v$. Therefore $F \cap F^{\prime}$ is contained in the Hilbert class field of $K$. This is enough to choose $v$. Any $v^{\prime}$ which lies in the same class as $(\alpha)$ in $C_{v}$ must be principal, and hence splits in $F \cap F^{\prime}$. We conclude that the splitting conditions are compatible, which proves the lemma.

3.2. Proof of Theorem 1. We assume in this section that $E$ has full level $P^{*}$ structure, and maintain the setup of $\S 2.5$. In particular, we have a fixed isomorphism

$$
\Phi:\left(K^{\times} / K^{\times P}\right)^{2} \cong H^{1}(K, E[P]) .
$$

Let $v=(\pi)$ and $v^{\prime}=\left(\pi^{\prime}\right)$ satisfy conditions (SC1)-(SC4). Put

$$
\xi:=\Phi\left(\pi^{P / D}, \pi^{\prime}\right) \in H^{1}(K, E[P]),
$$

so by Theorem 10 we have

$$
\Delta_{P}(\xi)=\left\langle\pi^{P / D}, \pi^{\prime}\right\rangle_{P} \in \operatorname{Br}(K) .
$$

Observe that $\Delta_{P}(\xi)$ is locally trivial away from $\pi$ and $\pi^{\prime}$. Indeed, by condition (SC3), the norm-residue symbol is trivial at the Archimedean places and at the places of residue characteristic dividing $P$. At all other places the norm residue symbol is "tame" and hence vanishes locally at $w$ when evaluated on a pair of $w$-adic units.

Let $C$ be the genus-one curve corresponding to the image $\eta$ of $\xi$ in $H^{1}(K, E)[P]$. Certainly the period of $\eta$ divides $P$. Suppose that the period of $\eta$ is less than $P$; then (since $p^{a} \eta=0$ ) it has period $P^{\prime}$ for some proper divisor $P^{\prime}$ of $P: P^{\prime} \xi=\imath_{P}(x)$. Then $l_{P}(x)$ is unramified at $\pi^{\prime}$ [Silverman 1986, Proposition VIII.2.1], whereas $P^{\prime} \xi=\left(\pi^{P P^{\prime} / D},\left(\pi^{\prime}\right)^{P^{\prime}}\right)$ is ramified at $\pi^{\prime}$, a contradiction. So $C$ has period $P$. Moreover, by Proposition 6,

$$
\Delta_{P D} j_{D}(\xi)=D \Delta_{P}(\xi)=D\left\langle\pi^{P / D}, \pi^{\prime}\right\rangle_{P}=\left\langle\pi^{P}, \pi^{\prime}\right\rangle_{P}=0,
$$

so there exists a rational divisor of degree $P D$ on $C$ and $I(C) \mid P D$.

Coming now to the heart of the matter, we suppose that the index $I$ of $C$ strictly divides $P D$. Then, by Proposition 7 there exists some lift $v$ of $\eta$ to $H^{1}(K, E[I])$ (under (4) with $n=I$ ) such that $\Delta_{I}(v)=0$. On the other hand, the local-at- $\pi$ norm-residue symbol $\left\langle\pi^{P / D}, \pi^{\prime}\right\rangle_{P, \pi}$ has exact order $D$, since, by condition (SC4), the corresponding central simple algebra trivializes over the Brauer group of an extension $L / K_{v}$ if and only if $\pi^{\prime}$ is a norm from the extension $L\left(\pi^{1 / D}\right) / L$ if and 
only if $D \mid e(L / K)$. Therefore the global norm-residue symbol $\left\langle\pi^{P / D}, \pi^{\prime}\right\rangle_{P}=$ $\Delta_{P}(\xi)$ has order at least $D$; since $I / P<D$ we must have

$$
0 \neq(I / P) \cdot \Delta_{P}(\xi)=\Delta_{I}\left(j_{I / P}(\xi)\right) .
$$

For the remainder of the proof we shall abbreviate $j_{I / P}(\xi)$ to $j(\xi)$. The classes $j(\xi)$ and $v \in H^{1}(K, E[I])$ are both lifts of $\eta$, so there exists $x \in E(K)$ with

$$
\iota_{I}(x)=v-j(\xi)
$$

Applying $\Delta$, we get

$$
0=\Delta_{I}(v)=\Delta_{I}(j(\xi))+\operatorname{Li}(j(\xi), x) .
$$

Now recall that $(\pi)$ splits completely in $K\left([P]^{-1} E(K)\right)$ by condition (SC2). This forces $E(K)$ to be divisible by $P$ in $E\left(K_{v}\right)$, and in particular $x \in P E\left(K_{v}\right)$. It follows that the $(\pi)$-component of $\operatorname{Li}(j(\xi), x)$ and hence also of $\Delta_{I}(j(\xi))$ are trivial. Thus $\Delta_{I}(j(\xi))=(I / P) \Delta_{P}(\xi)$ is locally trivial at all places except possibly at $\left(\pi^{\prime}\right)$, and by the reciprocity law and Hasse principle in the Brauer group of a local field this implies that it is globally trivial $-\Delta_{I}(j(\xi))=0-$ a contradiction.

Finally, we claim that the image $\eta$ of $\xi$ under $H^{1}(K, E[P]) \rightarrow H^{1}(K, E)[P]$ is locally trivial away from $v$ and $v^{\prime}$. First let $w$ be a bad prime. Then, by construction, $\pi, \pi^{\prime} \in K_{w}^{\times P}$ so $\left.\xi\right|_{K_{w}}=0$; a fortiori $\eta_{w}=0$. Now suppose $w \neq v, v^{\prime}$ is a good prime. Let $K_{w}^{\text {unr }}$ be the maximal unramified extension of $K_{w}$. Recall that the restriction map $H^{1}\left(K_{w}, E\right)[P] \rightarrow H^{1}\left(K_{w}^{\text {unr }}, E\right)[P]$ is injective [Lang and Tate 1958, Corollary 1]; this follows, for instance from the triviality of WC-groups over finite fields together with the fact that formation of the Néron model of a genus-one curve commutes with unramified base change. Since $K_{w}\left(\left(\pi^{\prime}\right)^{1 / P}\right) / K_{w}$ is unramified, $\xi$ trivializes over $K_{w}^{\text {unr }}$. But this implies that $\left.\zeta\right|_{K_{w}} ^{\text {unr }}=0$ and hence that $\left.\eta\right|_{K_{w}}=0$. This completes the proof of Theorem 1 .

3.3. Proof of Theorem 2: preliminaries. First, we wish to reduce to Theorem 1, that is, to the case where $E\left[P^{*}\right]$ has trivial Galois module structure. To this end we introduce the splitting field $K_{P}=K\left(E\left[P^{*}\right]\right)$ of the $P^{*}$-torsion. We will construct classes $\theta_{n}$ in $H^{1}\left(K_{P}, E[P]\right)$ in a similar manner as in the proof of Theorem 1, then we will set $\xi_{n}=\operatorname{cores}_{K_{P} / K} \theta_{n}$, and let $\eta_{n}$ be the image of $\xi_{n}$ in $H^{1}(K, E)$. In order to prove that the $\eta_{n}$ have the right properties, we will need to compute $\operatorname{res}_{K_{P} / K} \xi_{n}=$ res o cores $\theta_{n}$ explicitly.

In the following, let $\langle$,$\rangle denote the P$-Hilbert symbol on $\left(K_{P} \times / K_{P} \times P\right)^{2}$.

3.4. Proof of Theorem 2: choosing pairs of primes. In this section, we choose pairs of primes in a similar manner as in Lemma 13. The main difference is that we wish to choose an infinite sequence of pairs of primes $v_{i}, v_{i}^{\prime}$ in $K_{P}$ inductively. We 
will require conditions which are similar, and in some cases identical, to (SC1)(SC4). These conditions are as follows:

$\left(\mathrm{SC}^{\prime}\right)$ The primes $v_{i}=\left(\pi_{i}\right)$ and $v_{i}^{\prime}=\left(\pi_{i}^{\prime}\right)$ are principal, with totally positive generators $\pi_{i}$ and $\pi_{i}^{\prime}$.

$\left(\mathrm{SC} 2^{\prime}\right)$ Let $\tilde{v}_{i}$ and $\tilde{v}_{i}^{\prime}$ be primes of $K$ lying below $v_{i}$ and $v_{i}^{\prime}$ respectively (for fixed $i)$. Then all elements of $E(K)$ are $P$-divisible in $E\left(K_{\tilde{v}_{i}}\right)$ and in $E\left(K_{\tilde{v}_{i}^{\prime}}\right)$.

$\left(\mathrm{SC} 3^{\prime}\right)$ The generators $\pi_{i}$ and $\pi_{i}^{\prime}$ lie in $\left(K_{P}\right)_{w}^{\times P}$ for all bad primes $w$, primes lying above $S_{K}$, and for $w=v_{j}, v_{j}^{\prime}$ where $j<i$.

(SC4') The order of the image of $\pi_{i}^{\prime}$ in $\left(K_{P}\right)_{v_{i}}^{\times} /\left(K_{P}\right)_{v_{i}}^{\times P}$ is $P$. Additionally, $\sigma \pi_{i}^{\prime}$ lies in $\left(K_{P}\right)_{v_{i}}^{\times P}$ for all nontrivial $\sigma \in \operatorname{Gal}\left(K_{P} / K\right)$.

(SC5') The primes $\tilde{v}_{i}, \tilde{v}_{i}^{\prime}$ are totally split in $K_{P}$.

Lemma 14. There exist $v_{i}=\left(\pi_{i}\right), v_{i}^{\prime}=\left(\pi_{i}^{\prime}\right)$ satisfying conditions $\left(\mathrm{SC}^{\prime}\right)-\left(\mathrm{SC}^{\prime}\right)$.

Proof. We argue inductively: suppose that we have chosen $v_{j}, v_{j}^{\prime}$ for $j<i$. We let $\mathfrak{m}$ be the modulus given by the product of all bad primes in $K, P^{2}$, and all $\sigma v_{j}$ and $\sigma v_{j}^{\prime}$ for $j<i, \sigma \in \operatorname{Gal}\left(K_{P} / K\right)$; and let $F$ be the compositum of $K_{P}\left([P]^{-1} E(K)\right)$ and the $\mathfrak{m}$-ray class field of $K_{P}$. Note that $\mathfrak{m}$ is rational over $K$, so $F$ is Galois over $K$. As before, $F$ is an abelian extension of $K_{P}$. By the Chebotarev density theorem, there exists a prime $\tilde{v}_{i}$ of $K$ which splits completely in $F$. Let $v_{i}$ be any prime of $K_{P}$ which lies over $\tilde{v}_{i}$. Then, provided (SC5') holds, the same reasoning as in Lemma 13 shows that $v_{i}$ satisfies all the conditions. (We need (SC5') only for condition $\left(\mathrm{SC}^{\prime}\right)$, for otherwise we know only that $E\left(K_{P}\right)$ is $P$-divisible in $E\left(\left(K_{P}\right)_{v_{i}}\right)$.)

Let $\beta$ be a unit in $\left(K_{P}\right)_{v_{i}}$ which has order $P$ in $\left(K_{P}\right)_{v_{i}}^{\times} /\left(K_{P}\right)_{v_{i}}^{\times P}$. By the Chinese Remainder Theorem, there exists $\alpha \in K_{P}$ such that

$$
\begin{array}{ll}
\alpha \equiv \beta & \left(\bmod v_{i}\right), \\
\alpha \equiv 1 & \left(\bmod \sigma v_{i}\right) \quad \text { for all } \sigma \in \operatorname{Gal}\left(K_{P} / K\right), \sigma \neq 1 .
\end{array}
$$

Let $F^{\prime}$ be the ray class field for $K_{P}$ with modulus $\mathfrak{m}^{\prime}=\prod \sigma v_{i}$. Again, $\mathfrak{m}^{\prime}$ is rational over $K$, so that $F^{\prime}$ is Galois over $K$. Let $C_{\mathfrak{m}^{\prime}}$ be the class group for $K_{P}$ with modulus $\mathfrak{m}^{\prime}$. The Artin reciprocity map gives an isomorphism $C_{\mathfrak{m}^{\prime}} \rightarrow \operatorname{Gal}\left(F^{\prime} / K_{P}\right)$. Let $\gamma_{F^{\prime}}$ be the image of $(\alpha)$ under this isomorphism. Since $F \cap F^{\prime}$ is contained in the Hilbert class field of $K_{P}$ and $(\alpha)$ is principal, there exists $\gamma \in \operatorname{Gal}\left(F F^{\prime} / K_{P}\right)$ such that $\left.\gamma\right|_{F^{\prime}}=\gamma_{F^{\prime}}$ and $\left.\gamma\right|_{F}$ is the identity. Since $F F^{\prime}$ is Galois over $K$, we view $\operatorname{Gal}\left(F F^{\prime} / K_{P}\right)$ as a subgroup of $\operatorname{Gal}\left(F F^{\prime} / K\right)$. Let $[\gamma]$ be the conjugacy class of $\gamma$ in this larger Galois group. By Chebotarev, there exists a prime $\tilde{v}_{i}^{\prime}$ of $K$ such that any Frobenius associated to $\tilde{v}_{i}^{\prime}$ in the extension $F F^{\prime} / K$ lies in $[\gamma]$. Let $v_{i}^{\prime}$ be a prime of $K_{P}$ lying over $\tilde{v}_{i}^{\prime}$. By replacing $v_{i}^{\prime}$ by a conjugate if necessary, we 
may assume that the Frobenius of $v_{i}^{\prime}$ in the extension $F F^{\prime} / K_{P}$ is precisely $\gamma$ (the extension here is abelian, so saying "the" Frobenius makes sense). By the same arguments as in Lemma 13, $v_{i}^{\prime}$ satisfies the first three conditions.

One sees that $\pi_{i}^{\prime} \equiv \alpha\left(\bmod \left(\pi_{i}\right)\right)$, so that the order of $\pi_{i}^{\prime}$ in $\left(K_{P}\right)_{v_{i}}^{\times} /\left(K_{P}\right)_{v_{i}}^{\times P}$ is $P$. Also, $\pi_{i}^{\prime} \equiv 1\left(\bmod \left(\sigma \pi_{i}\right)\right)$ for nontrivial $\sigma$, so that $\sigma \pi_{i}^{\prime} \equiv 1 \bmod \left(\pi_{i}\right)$. Therefore $v_{i}^{\prime}$ satisfies condition $\left(\mathrm{SC}^{\prime}\right)$.

Any Frobenius associated to $\tilde{v}_{i}^{\prime}$ in the extension $K_{P} / K$ is trivial, so that $\tilde{v}_{i}^{\prime}$ splits in $K_{P}$, thus satisfying $\left(\mathrm{SC}^{\prime}\right)$.

3.5. Proof of Theorem 2: corestrictions. As in the proof of Theorem 1, a choice of basis for $E[P]$ yields an isomorphism

$$
\Phi:\left(K_{P}^{\times} / K_{P} \times P\right)^{2} \rightarrow H^{1}\left(K_{P}, E[P]\right) .
$$

Let $\theta_{n}$ be either $\Phi\left(\pi_{n}, \pi_{n}^{\prime}\right)$ or $\Phi\left(\pi_{n}, 1\right)$; that is, we will need to consider both cases. Let cores be the corestriction map

$$
H^{1}\left(K_{P}, E[P]\right) \rightarrow H^{1}(K, E[P]),
$$

and write $\xi_{n}=\operatorname{cores} \theta_{n}$. In order to prove Theorem 2, we would like to compute $\Delta_{P}\left(\xi_{n}-\xi_{m}\right)$ as well as the period of $\left(\xi_{n}-\xi_{m}\right)$. To do this, we will instead compute the obstruction and period of $\operatorname{res}\left(\xi_{n}-\xi_{m}\right)$, where res is the restriction map

$$
H^{1}(K, E[P]) \rightarrow H^{1}\left(K_{P}, E[P]\right) .
$$

Both res and cores are $\mathbb{Z}$-linear, so it will suffice to compute res o cores $\left(\Phi\left(\pi_{n}, 1\right)\right)$ and res $\circ \operatorname{cores}\left(\Phi\left(1, \pi_{n}^{\prime}\right)\right)$.

Let $\mathrm{Nm} \in \operatorname{End}\left(H^{1}\left(K_{P}, E[P]\right)\right)$ be given, on the level of cocycles, by

$$
\operatorname{Nm}(\theta)(\sigma)=\sum_{\bar{\gamma} \in \operatorname{Gal}\left(K_{P} / K\right)} \gamma \cdot \theta\left(\gamma^{-1} \sigma \gamma\right),
$$

where $\gamma$ is a fixed lift of $\bar{\gamma}$ to $\mathfrak{g}_{K}$. Since $E[P]$ is rational over $K_{P}$, there is a unique cocycle in each cohomology class, so that $\mathrm{Nm}$ is well-defined as an endomorphism of $H^{1}\left(K_{P}, E[P]\right)$.

Lemma 15. If $\theta \in H^{1}\left(K_{P}, E[P]\right)$, then res o $\operatorname{cores} \theta=\mathrm{Nm} \theta$.

Proof. The lemma follows from the definition of cores on $H^{0}\left(K_{P}, E[P]\right)$ and dimension shifting; see for example [Serre 1962, p. 119].

In the remainder of this section, we drop the subscript $n$.

Lemma 15 shows that res o cores $(\Phi(\pi, 1))=\operatorname{Nm}(\Phi(\pi, 1))$. Unfortunately, $\mathrm{Nm}$ and $\Phi$ do not commute, as the Galois actions on $E[P]$ and $\mu_{P} \times \mu_{P}$ differ. The 
representation on $E[P]$ gives, with respect to our fixed basis, a homomorphism

$$
\begin{aligned}
\operatorname{Gal}\left(K_{P} / K\right) & \rightarrow \mathrm{GL}_{2}(\mathbb{Z} / P \mathbb{Z}) \\
\sigma & \mapsto M_{\sigma}=\left(\begin{array}{ll}
i(\sigma) & j(\sigma) \\
k(\sigma) & \ell(\sigma)
\end{array}\right) .
\end{aligned}
$$

Then we have

Proposition 16. Let $\sigma \in \operatorname{Gal}\left(K_{P} / K\right)$ and $(a, b) \in\left(K_{P} \times / K_{P} \times P\right)^{2}$. Then

$$
\Phi(a, b)^{\sigma}=\Phi\left(\frac{M_{\sigma}}{\operatorname{det} M_{\sigma}}(\sigma a, \sigma b)\right),
$$

where $M_{\sigma}(a, b)$ is given by the natural action of $\mathrm{GL}_{2}(\mathbb{Z} / P \mathbb{Z})$ on $\left(K_{P} \times / K_{P} \times P\right)^{2}$; that is, $M_{\sigma}(a, b)=\left(a^{i(\sigma)} b^{j(\sigma)}, a^{k(\sigma)} b^{\ell(\sigma)}\right)$.

Proof. Our choice of basis for $E[P]$ gives rise to a group isomorphism

$$
\rho: E[P] \rightarrow \mu_{P} \times \mu_{P} .
$$

Define a $\mathbb{Z}\left[\operatorname{Gal}\left(K_{P} / K\right)\right]$-module $\left(\mu_{P} \times \mu_{P}\right)_{\rho}$ which, as a $\mathbb{Z}$-module, is $\mu_{P} \times \mu_{P}$, but which possesses a Galois structure making $\rho$ into a $\operatorname{Gal}\left(K_{P} / K\right)$-equivariant map. In particular, if $\left(\zeta_{1}, \zeta_{2}\right) \in\left(\mu_{P} \times \mu_{P}\right)_{\rho}$ and $\sigma \in \operatorname{Gal}\left(K_{P} / K\right)$, we have

$$
\rho \circ \sigma \circ \rho^{-1}\left(\zeta_{1}, \zeta_{2}\right)=\sigma\left(\zeta_{1}, \zeta_{2}\right)=M_{\sigma}\left(\zeta_{1}, \zeta_{2}\right) .
$$

On the other hand, for $\left(\zeta_{1}^{\prime}, \zeta_{2}^{\prime}\right) \in \mu_{P} \times \mu_{P}$ the Galois action is

$$
\sigma\left(\zeta_{1}^{\prime}, \zeta_{2}^{\prime}\right)=\operatorname{det} M_{\sigma} \cdot\left(\zeta_{1}^{\prime}, \zeta_{2}^{\prime}\right)
$$

where the action on the right is the diagonal action of $\mathbb{Z} / P \mathbb{Z}$.

Let $i: \mu_{P} \times \mu_{P} \rightarrow\left(\mu_{P} \times \mu_{P}\right)_{\rho}$ be the canonical group isomorphism; it does not respect the $\operatorname{Gal}\left(K_{P} / K\right)$-action. If $A$ is any $\mathfrak{g}_{K_{P}}$-module, write $H^{1}(A)$ for $H^{1}\left(K_{P}, A\right)$. Then $i$ induces a map

$$
i_{*}: H^{1}\left(\mu_{P} \times \mu_{P}\right) \rightarrow H^{1}\left(\left(\mu_{P} \times \mu_{P}\right)_{\rho}\right) .
$$

Let $M$ be either $\left(\mu_{P} \times \mu_{P}\right)_{\rho}$ or $\mu_{P} \times \mu_{P}$. Since in either case $M$ is a trivial $\mathfrak{g}_{K_{P}}$-module, the set of coboundaries $B^{1}\left(K_{P}, M\right)$ is zero, and so $H^{1}\left(K_{P}, M\right)=$ $Z^{1}\left(K_{P}, M\right)$, the set of 1-cocycles from $\mathfrak{g}_{K_{P}}$ to $M$. We can therefore identify cohomology classes with cocycles in both cases.

Consider the commutative diagram

$$
\left(K_{P} \times K_{P} \times P\right)^{2} \stackrel{\psi}{\longrightarrow} H_{\psi_{\rho}}^{H^{1}\left(\mu_{P} \times \mu_{P}\right)} \underbrace{\longrightarrow}_{H^{1}\left(\left(\mu_{P} \times \mu_{P}\right)_{\rho}\right) \underset{\lambda}{i_{*}} H^{1}(E[P])}
$$


The horizontal maps are $\operatorname{Gal}\left(K_{P} / K\right)$-isomorphisms. The map $\lambda$ is induced by $(i \circ \rho)^{-1}$, and $\psi$ is the Kummer map. The diagonal map $\psi_{\rho}$ is $\psi \circ i_{*}$. Thus, $\Phi=\lambda \circ \psi_{\rho}$. Note that $\mathfrak{g}_{K}$ acts on all of the groups in (11) through its quotient $\operatorname{Gal}\left(K_{P} / K\right)$. Let $\gamma$ be an element of $\mathfrak{g}_{K_{P}}$ and $\sigma$ an element of $\mathfrak{g}_{K}$. Then

$$
\begin{aligned}
{\left[\psi_{\rho}(a, b)\right]^{\sigma}(\gamma) } & =\left[i_{*} \psi(a, b)\right]^{\sigma}(\gamma) \\
& =\sigma\left[i\left(\psi(a, b)\left(\sigma^{-1} \gamma \sigma\right)\right)\right] \\
& =\sigma\left[i\left(\sigma^{-1} \sigma \psi(a, b)\left(\sigma^{-1} \gamma \sigma\right)\right)\right] \\
& =\sigma\left[i\left(\sigma^{-1} \psi(\sigma a, \sigma b)(\gamma)\right)\right] \\
& =M_{\sigma}\left[\left(i\left(\operatorname{det} M_{\sigma}^{-1} \cdot \psi(\sigma a, \sigma b)(\gamma)\right)\right]\right. \\
& =\frac{M_{\sigma}}{\operatorname{det} M_{\sigma}}[i(\psi(\sigma a, \sigma b)(\gamma))] \\
& =\frac{M_{\sigma}}{\operatorname{det} M_{\sigma}} \psi_{\rho}(\sigma a, \sigma b)(\gamma) .
\end{aligned}
$$

Applying $\lambda$ on both sides, we obtain the result.

Corollary 17. We have

$$
\mathrm{Nm} \Phi((a, b))=\Phi\left(\prod \frac{1}{\operatorname{det} M_{\sigma}}\left(\sigma a^{i(\sigma)} \sigma b^{j(\sigma)}, \sigma a^{k(\sigma)} \sigma b^{\ell(\sigma)}\right)\right),
$$

where the product extends over all $\sigma \in \operatorname{Gal}\left(K_{P} / K\right)$ and is taken component-wise. Let $(c, d)=\Phi^{-1} \mathrm{Nm} \Phi(\pi, 1)$ and $\left(c^{\prime}, d^{\prime}\right)=\Phi^{-1} \mathrm{Nm} \Phi\left(1, \pi^{\prime}\right)$.

Lemma 18. Let $v$ be the place of $K_{P}$ corresponding to $\pi$. Either $\operatorname{order}\left(\langle c, d\rangle_{v}\right)=$ $P$ or $\operatorname{order}\left(\left\langle c c^{\prime}, d d^{\prime}\right\rangle_{v}\right)=P$.

Proof. If order $(\langle c, d\rangle)=P$, then we are done. So suppose that $\operatorname{order}(\langle c, d\rangle)<P$. In fact, since $P$ is a prime power, the order strictly divides $P$.

Expanding out the Hilbert symbol, we get

$$
\left\langle c c^{\prime}, d d^{\prime}\right\rangle=\langle c, d\rangle+\left\langle c, d^{\prime}\right\rangle+\left\langle c^{\prime}, d\right\rangle+\left\langle c^{\prime}, d^{\prime}\right\rangle .
$$

We have $\left\langle c^{\prime}, d\right\rangle_{v}=\left\langle c^{\prime}, d^{\prime}\right\rangle_{v}=0$ since all are $v$-adic units. By our assumption at the start of the proof, $\langle c, d\rangle_{v}$ has order strictly dividing $P$. That leaves $\left\langle c, d^{\prime}\right\rangle_{v}$. By Corollary 17 ,

$$
d^{\prime}=\pi^{\prime} \cdot \prod_{\sigma \neq 1}\left(\sigma \pi^{\prime}\right)^{e_{\sigma}}
$$

for some integers $e_{\sigma}$. Our choice of $\pi^{\prime}$ implies that $\pi^{\prime} \equiv \alpha(\bmod (\pi))$, where $\alpha$ was chosen to have order $P$ in $K_{v}^{\times P}$, while $\sigma \pi^{\prime} \equiv 1(\bmod (\pi))$ for nontrivial $\sigma$ (see $(10))$. Thus $d^{\prime} \equiv \alpha(\bmod (\pi))$. Therefore $K_{v}\left(d^{\prime 1 / P}\right) / K$ is the unramified extension of degree $P$. (Equivalently, we may appeal to condition $\left(\mathrm{SC}^{\prime}\right)$.) 
We now use similar reasoning as in the proof of Theorem 1 to see that $\left\langle\pi, d^{\prime}\right\rangle_{v}$ has order $P$. Since $v(c)=1$, the order of $\left\langle c, d^{\prime}\right\rangle_{v}$ is exactly $P$. This shows $\left\langle c c^{\prime}, d d^{\prime}\right\rangle_{v}$ has exact order $P$.

If $\langle c, d\rangle$ has order $P$, let $\theta=\Phi(\pi, 1)$, so that $\xi=\operatorname{cores} \theta$ satisfies

$$
\operatorname{res} \xi=\operatorname{Nm} \Phi(\pi, 1)=\Phi(c, d) ;
$$

otherwise, let $\theta=\Phi\left(\pi, \pi^{\prime}\right)$, so that res $\xi=\Phi\left(c c^{\prime}, d d^{\prime}\right)$. Let $(a, b)$ denote whichever pair we've chosen, $(c, d)$ or $\left(c c^{\prime}, d d^{\prime}\right)$.

Let us now reintroduce subscripts, so that

$$
\begin{aligned}
\xi_{n} & =\operatorname{cores} \theta_{n} \\
& =\left\{\begin{array}{l}
\operatorname{cores} \Phi\left(\pi_{n}, \pi_{n}^{\prime}\right) \text { or } \\
\operatorname{cores} \Phi\left(\pi_{n}, 1\right)
\end{array}\right. \\
\left(a_{n}, b_{n}\right) & =\Phi^{-1} \operatorname{res} \xi_{n} .
\end{aligned}
$$

Lemma 19. Let $0 \leq m<n$. Then $\Delta_{P}\left(\operatorname{res}\left(\xi_{m}-\xi_{n}\right)\right)$ has order $P$ at $v_{m}$.

Proof. Write $v$ for $v_{m}$. Since $E\left[P^{*}\right] \subset E\left(K_{P}\right)$, the obstruction map can be computed using the Hilbert symbol. Thus we wish to compute the order of

$$
\left\langle\frac{a_{m}}{a_{n}}, \frac{b_{m}}{b_{n}}\right\rangle_{v} .
$$

By the bilinearity of the Hilbert symbol, it suffices to compute

$$
\left\langle a_{m}, b_{m}\right\rangle_{v}-\left\langle a_{m}, b_{n}\right\rangle_{v}-\left\langle a_{n}, b_{m}\right\rangle_{v}+\left\langle a_{n}, b_{n}\right\rangle_{v} .
$$

By Lemma 18, the first term has order $P$. Since $a_{n}, b_{m}$ and $b_{n}$ are all units at $v$, the last two terms are zero. That leaves the term $\left\langle a_{m}, b_{n}\right\rangle_{v}$. By Corollary $17, b_{n}$ is a product of $\sigma \pi_{n}$ and $\sigma \pi_{n}^{\prime}$. By condition $\left(\mathrm{SC}^{\prime}\right)$, these all lie in $K_{v}^{\times P}$. Therefore the second term is also zero. The Lemma follows.

3.6. Proof of Theorem 2: conclusion. Let $C$ be the curve represented by the class $\xi:=\xi_{i}-\xi_{j}$ for some $i \neq j$. Clearly, $P(C) \mid P$. If we can show that $I(C)=P^{2}$, then by (1) we must have $P(C)=P$.

Since $E[P] \subset E\left(K_{P}\right)$ (and $E[2 P] \subset E\left(K_{P}\right)$ when $P$ is even), the obstruction map on $H^{1}\left(K_{P}, E[P]\right)$ is given by the Hilbert symbol. In view of Lemma 19, $\Delta_{P}\left(\operatorname{res}_{K_{P} / K} \xi\right)$ has order $P$ at $v_{i}$. Therefore $\Delta_{P}(\xi)$ has order $P$ at the prime $w$ satisfying $v_{i} \mid w$.

Suppose that $C$ has index $P \cdot D$ for some $D \mid P$. Then there exists some $\eta \in$ $H^{1}(K, E[P D])$ representing $C$ such that $\Delta_{P D}(\eta)=0$. Let $j_{D}$ be the natural map $H^{1}(K, E[P]) \rightarrow H^{1}(K, E[P D])$. The classes $\eta$ and $j_{D}(\xi)$ represent the same 
curve $C$, so there exists some $x \in E(K)$ such that $\eta=j_{D}(\xi)+\imath_{P D}(x)$. Since $\Delta_{P D}\left(l_{P D}(x)\right)=0$, by the remarks at the start of Section 2.1,

$$
\Delta_{P D}(\eta)=\Delta_{P D}\left(j_{D}(\xi)\right)+\operatorname{Li}(\eta, x) .
$$

Recall that $\operatorname{Li}(\eta, x)$ is the Tate pairing. Let us consider this equality locally, at $w$. The left-hand side is zero by hypothesis. By condition (SC2'), $x$ lies in $P \cdot E\left(K_{w}\right)$. Since $P(C) \mid P$, the Tate pairing at $w$ is trivial. Hence $\Delta_{P D}\left(j_{D}(\xi)\right)$ must be zero at $w$. But by Proposition 6,

$$
\Delta_{P D}\left(j_{D}(\xi)\right)=D \Delta_{P}(\xi) .
$$

We showed earlier that $\Delta_{P}(\xi)$ has order $P$ at $w$. Therefore $D=P$, and so $I(C)=$ $P^{2}$.

Let $\eta_{i}$ be the image of $\xi_{i}$ in $H^{1}(K, E)$. It remains to show that res $\eta_{v}=0$ for $v \in S_{K}$. Recall that $\eta_{i}=\operatorname{cores} \Phi\left(\pi_{i}, 1\right)$ or cores $\Phi\left(\pi_{i}, \pi_{i}^{\prime}\right)$. For $w \mid v$ a place of $K_{P}$, the proof of Theorem 1 showed that the curves corresponding to $\Phi\left(\pi_{i}, 1\right)$ and $\Phi\left(\pi_{i}, \pi_{i}^{\prime}\right)$ were trivial at $w$. But the corestriction map induces a homomorphism

$$
\oplus_{w \mid v} H^{1}\left(\left(K_{P}\right)_{w}, E\right) \rightarrow H^{1}\left(K_{v}, E\right)
$$

which proves that $\eta_{i}$ is trivial at $v$. This completes the proof of Theorem 2 .

3.7. Proof of Theorem 3. Recall the following two "classical" instances of period equals index.

(i) [Lang and Tate 1958] $F$ is the completion of a global field at a place $v$, $E=\operatorname{Jac}(C)$ has good reduction, and $v$ does not divide the period of $C$.

(ii) [Cassels 1963] $F$ is global and $C \in \amalg(F, E)$.

Note that Lichtenbaum showed that $P=I$ for all genus-one curves defined over the completion of a global field. However, the result of Lang and Tate, apart from being more elementary, is also more precise: they show also that a finite extension field $F^{\prime} / F$ splits a genus-one curve $C_{/ K}$ if and only if the period $P$ of $C$ divides the relative ramification index $e\left(F^{\prime} / F\right)$. This will be used in the proof.

Take $S$ to be the union of the infinite places, the finite places which divide $P$ and the places of bad reduction for $E$. Let $\left\{\eta_{i}\right\}_{i=0}^{\infty}$ be the sequence of classes constructed in Theorem 2. We will show that for any positive integer $r$, there exists a degree $P$ field extension $L / K$ such that the restrictions of $\eta_{1}, \ldots, \eta_{r}$ to $L$ are pairwise distinct, locally trivial, and of period $P$.

Indeed, let $S_{r}=\bigcup_{i=1}^{r} \operatorname{supp}\left(\eta_{i}\right)$. We have $S_{r} \cap S=\varnothing$, so that each $v_{i} \in S_{r}$ is a finite place of good reduction for $E$ and residue characteristic prime to $P$.

For each $v_{i} \in S_{r}$, let $L_{i} / K_{v_{i}}$ be a totally ramified extension of degree $P$. There exists a degree $P$ global extension $L=L(r)$ of $K$ such that for all $v_{i} \in S_{r}, L \otimes_{K}$ 
$K_{v_{i}} \cong L_{i} \cdot{ }^{3}$ By the results (i) of Lang and Tate cited above, $\left.\eta_{i}\right|_{L}$ is locally trivial. Moreover, since $\eta_{i}=\eta_{i}-\eta_{0}$ has index $P^{2}$ and $L / K$ is a degree $P$ extension, $I\left(\left.\eta_{i}\right|_{L}\right) \geq P$. But on the other hand, by (ii) above, $I\left(\left.\eta_{i}\right|_{L}\right)=P\left(\left.\eta_{i}\right|_{L}\right) \mid P\left(\eta_{i}\right)=P$, so for all $i, 1 \leq i \leq r,\left.\eta_{i}\right|_{L}$ has period and index equal to $P$.

The only worry is that their restrictions are not distinct. But suppose that $\left.\eta_{i}\right|_{L}=$ $\left.\eta_{j}\right|_{L}$. Then $\eta_{i}-\eta_{j}$ would lie in the kernel $\mathrm{res}_{L}$. This would imply that $I\left(\eta_{i}-\eta_{j}\right) \mid P$, which we have arranged not to be the case.

3.8. Remarks about ramification. The proof of Theorem 3 differs from that of [Clark 2005, Theorem 1] in that we explicitly make use of extensions $L / K$ that are ramified at many primes. Given our strategy of proof, this is unavoidable: using (i), the number of order $P$ elements in $\operatorname{res}_{L}\left(H^{1}(K, E)\right) \cap \amalg(L, E)$ can be bounded in terms of the number of ramified primes of $L / K$. It is interesting to ask whether this same boundedness result holds for order $P$ elements in $\amalg(L, E)$, and conversely, whether the number of order $P$ elements of $\amalg(L, E)$ necessarily approaches infinity with the number of ramified primes.

Both of these questions have affirmative answers when $P=2$, according to work of Yu [2004]. Given a quadratic extension $L / K$, Yu computes the order of the kernel and cokernel of the natural map $\amalg(K, E) \oplus \amalg\left(K, E^{\chi}\right) \rightarrow \amalg(L, E)$; here $E^{\chi}$ is the twist of $E_{/ K}$ by the quadratic character $\chi$ of $L / K$. In particular, one can deduce Theorem 3 for $P=2$ from Yu's work, with one caveat: his analysis is conditional on the finiteness of $\amalg(K, E)$. That the existence of an infinite subgroup of $\amalg(K, E)$ would hamper our ability to show that $\amalg(L, E)[2]$ is large is somewhat curious, but seems to be the true state of affairs.

The consistency of Theorem 3 with the results of [Yu 2004] might thus be regarded as some confirmatory evidence for the finiteness of Shafarevich-Tate groups. How seriously such evidence ought to be taken is, of course, up to the reader to decide.

\section{References}

[Bölling 1975] R. Bölling, "Die Ordnung der Schafarewitsch-Tate-Gruppe kann beliebig groß werden”, Math. Nachr. 67 (1975), 157-179. MR 52 \#5684

[Bosch et al. 1990] S. Bosch, W. Lütkebohmert, and M. Raynaud, Néron models, Ergebnisse der Math. 21, Springer, Berlin, 1990. MR 91i:14034 Zbl 0705.14001

[Cassels 1962] J. W. S. Cassels, "Arithmetic on curves of genus 1, III: The Tate-Šafarevič and Selmer groups", Proc. London Math. Soc. (3) 12 (1962), 259-296. MR 29 \#1212 Zbl 0106.03705

[Cassels 1963] J. W. S. Cassels, "Arithmetic on curves of genus 1, V: Two counterexamples", J. London Math. Soc. 38 (1963), 244-248. MR 26 \#6171 Zbl 0113.03701

[Cassels 1964] J. W. S. Cassels, "Arithmetic on curves of genus 1, VI: The Tate-Šafarevič group can be arbitrarily large", J. Reine Angew. Math. 214/215 (1964), 65-70. MR 29 \#104 Zbl 0236.14012

\footnotetext{
${ }^{3}$ This is a standard weak approximation/Krasner's Lemma argument [Clark 2005, p. 2].
} 
[Ciperiani and Krashen 2007] M. Ciperiani and D. Krashen, "Relative Brauer groups of genus 1 curves", preprint, 2007. arXiv math/0701614

[Clark 2005] P. L. Clark, "The period-index problem in WC-groups, I: Elliptic curves", J. Number Theory 114:1 (2005), 193-208. MR 2006f:11059 Zbl 1087.11036

[Clark 2006a] P. L. Clark, "Period-index problems in WC-groups, II: Abelian varieties", preprint, 2006, Available at http://math.uga.edu/ pete/wc2.pdf.

[Clark 2006b] P. L. Clark, "There are genus one curves of every index over every number field", $J$. Reine Angew. Math. 594 (2006), 201-206. MR 2007b:11080 Zbl 1097.14024

[Cremona et al. 2008] J. E. Cremona, T. A. Fisher, C. O'Neil, D. Simon, and M. Stoll, "Explicit n-descent on elliptic curves. I. Algebra”, J. Reine Angew. Math. 615 (2008), 121-155. MR 2009g: 11067

[Donnelly 2003] S. Donnelly, "Elements of given order in Tate-Shafarevich groups of elliptic curves", 2003.

[Fisher 2001] T. Fisher, "Some examples of 5 and 7 descent for elliptic curves over Q", J. Eur. Math. Soc. (JEMS) 3:2 (2001), 169-201. MR 2002m:11045 Zbl 1007.11031

[Kloosterman 2005] R. Kloosterman, "The $p$-part of the Tate-Shafarevich groups of elliptic curves can be arbitrarily large”, J. Théor. Nombres Bordeaux 17:3 (2005), 787-800. MR 2006k:11102 Zbl 1153.11313

[Kloosterman and Schaefer 2003] R. Kloosterman and E. F. Schaefer, "Selmer groups of elliptic curves that can be arbitrarily large", J. Number Theory 99:1 (2003), 148-163. MR 2003m:11081 Zbl 1074.11032

[Lang and Tate 1958] S. Lang and J. Tate, "Principal homogeneous spaces over abelian varieties", Amer. J. Math. 80 (1958), 659-684. MR 21 \#4960 Zbl 0097.36203

[Lichtenbaum 1968] S. Lichtenbaum, “The period-index problem for elliptic curves", Amer. J. Math. 90 (1968), 1209-1223. MR 38 \#5788 Zbl 0187.18602

[Lichtenbaum 1969] S. Lichtenbaum, "Duality theorems for curves over p-adic fields", Invent. Math. 7 (1969), 120-136. MR 39 \#4158 Zbl 0186.26402

[Matsuno 2007] K. Matsuno, "Construction of elliptic curves with large Iwasawa $\lambda$-invariants and large Tate-Shafarevich groups”, Manuscripta Math. 122:3 (2007), 289-304. MR 2008h:11106 Zbl 1152.11045

[Milne 1972] J. S. Milne, "Addendum to 'Weil-Châtelet groups over local fields' (Ann. Sci. École Norm. Sup. (4) 3 (1970), 273-284)", Ann. Sci. École Norm. Sup. (4) 5 (1972), 261-264. MR 48 \#6121 Zbl 0241.14022

[Mumford 1966] D. Mumford, "On the equations defining abelian varieties. I", Invent. Math. 1 (1966), 287-354. MR 34 \#4269 Zbl 0219.14024

[Olson 1970] L. D. Olson, "Galois cohomology of cycles and applications to elliptic curves", Amer. J. Math. 92 (1970), 75-85. MR 41 \#8421 Zbl 0197.17301

[O’Neil 2002] C. O’Neil, “The period-index obstruction for elliptic curves”, J. Number Theory 95:2 (2002), 329-339. MR 2003f:11079 Zbl 1033.11029

[Serre 1962] J.-P. Serre, Corps locaux, Actualités Sci. Indust. 1296, Hermann, Paris, 1962. MR 27 \#133 Zbl 0137.02601

[Sharif 2006] S. Sharif, Construction of curves with prescribed period and index, Ph.D. thesis, University of California, Berkeley, 2006.

[Silverman 1986] J. H. Silverman, The arithmetic of elliptic curves, Graduate Texts in Mathematics 106, Springer, New York, 1986. MR 87g:11070 Zbl 0585.14026 
[Tate 1958] J. Tate, “WC-groups over p-adic fields”, Séminaire Bourbaki 1957/1958 (Exposé 156) 13, Secrétariat mathématique, Paris, 1958. Reprinted as pp. 265-277 in Séminaire Bourbaki 4, Soc. Math. France, Paris, 1995. MR 21 \#4162

[Yu 2004] H. Yu, “On Tate-Shafarevich groups over Galois extensions”, Israel J. Math. 141 (2004), 211-220. MR 2005d:14034 Zbl 1071.11033

[Zarhin 1974] J. G. Zarhin, "Noncommutative cohomology and Mumford groups", Mat. Zametki 15 (1974), 415-419. MR $50 \# 7090$

Communicated by Karl Rubin

Received 2009-01-15 Revised 2009-11-12 Accepted 2009-11-16

pete@math.uga.edu

University of Georgia, Department of Mathematics, Athens, GA 30602, United States http://www.math.uga.edu/ pete/

sharif@math.duke.edu

Department of Mathematics, Duke University, Durham, NC 27708, United States http://www. math.duke.edu/ sharif 


\title{
On the dimension of $\mathrm{H}$-strata in quantum algebras
}

\author{
Jason P. Bell and Stéphane Launois
}

\begin{abstract}
We study the topology of the prime spectrum of an algebra supporting a rational torus action. More precisely, we study inclusions between prime ideals that are torus-invariant using the $H$-stratification theory of Goodearl and Letzter on the one hand, and the theory of deleting derivations of Cauchon on the other. We also give a formula for the dimensions of the $H$-strata described by Goodearl and Letzter. We apply the results obtained to the algebra of $m \times n$ generic quantum matrices to show that the dimensions of the $H$-strata are bounded above by the minimum of $m$ and $n$, and that all values between 0 and this bound are achieved.
\end{abstract}

\section{Introduction}

We denote by $R=\mathscr{O}_{q}\left(M_{m, n}\right)$ the standard quantization of the ring of regular functions on $m \times n$ matrices with entries in a field $\mathbb{K}$; it is the $\mathbb{K}$-algebra generated by the $m \times n$ indeterminates $Y_{i, \alpha}, 1 \leq i \leq m$ and $1 \leq \alpha \leq n$, subject to the relations

$$
\begin{aligned}
& Y_{i, \beta} Y_{i, \alpha}=q^{-1} Y_{i, \alpha} Y_{i, \beta}, \quad \alpha<\beta, \\
& Y_{j, \alpha} Y_{i, \alpha}=q^{-1} Y_{i, \alpha} Y_{j, \alpha}, \quad i<j, \\
& Y_{j, \beta} Y_{i, \alpha}=Y_{i, \alpha} Y_{j, \beta}, \quad i<j, \alpha>\beta, \\
& Y_{j, \beta} Y_{i, \alpha}=Y_{i, \alpha} Y_{j, \beta}-\left(q-q^{-1}\right) Y_{i, \beta} Y_{j, \alpha}, \quad i<j, \alpha<\beta,
\end{aligned}
$$

where $q \in \mathbb{K}^{*}$ is not a root of unity. We note that the torus $\left(\mathbb{K}^{*}\right)^{m+n}$ acts on $R$ by $\mathbb{K}$-algebra automorphisms via the action

$$
\left(a_{1}, \ldots, a_{m}, b_{1}, \ldots, b_{n}\right) \cdot Y_{i, \alpha}=a_{i} b_{\alpha} Y_{i, \alpha} \quad \text { for } 1 \leq i \leq m, 1 \leq \alpha \leq n .
$$

Understanding this torus action has been responsible for most of the important advances that have been made in the study of quantum matrices. The most important object of study is the prime spectrum of $R$. In analogy with algebraic

MSC2000: primary 16W35; secondary 20G42.

Keywords: prime spectrum, Zariski topology, stratification, quantum matrices.

Bell thanks NSERC for its generous support. Launois's research was supported by a Marie Curie European Reintegration Grant within the 7th European Community Framework Programme. 
geometry, where great understanding of commutative rings comes from the study of their prime spectra, one seeks to understand the prime spectrum of $R$ and its topology. The noncommutativity introduced by the parameter $q$ in quantum matrices makes the prime spectrum of $R$ harder to understand than the prime spectrum of the coordinate ring of the variety of $m \times n$ matrices and much work has been done in understanding the structure of this topological object. The most important of these advances is the stratification theory of Goodearl and Letzter [2000].

To describe the work of those authors, we give a few basic definitions. Let $A$ be a $\mathbb{K}$-algebra with a group $H$ acting on it by $\mathbb{K}$-algebra automorphisms. A twosided ideal $I$ of $A$ is said to be $H$-invariant if $h \cdot I=I$ for all $h \in H$. An $H$-prime ideal of $A$ is a proper $H$-invariant ideal $J$ of $A$ such that whenever $J$ contains the product of two $H$-invariant ideals of $A, J$ contains at least one of them. We denote by $H$-Spec $(A)$ the set of all $H$-prime ideals of $A$. Observe that if $P$ is a prime ideal of $A$ then

$$
(P: H):=\bigcap_{h \in H} h \cdot P
$$

is an $H$-prime ideal of $A$. This observation allowed Goodearl and Letzter [2000] (see also [Brown and Goodearl 2002]) to construct a stratification of the prime spectrum of $A$ that is indexed by the $H$-spectrum. Indeed, let $J$ be an $H$-prime ideal of $A$. We denote by $\operatorname{Spec}_{J}(A)$ the $H$-stratum associated to $J$; that is,

$$
\operatorname{Spec}_{J}(A)=\{P \in \operatorname{Spec}(A) \mid(P: H)=J\} .
$$

Then the $H$-strata of $\operatorname{Spec}(A)$ form a partition of $\operatorname{Spec}(A)$ [Brown and Goodearl 2002, Chapter II.2]; that is,

$$
\operatorname{Spec}(A)=\bigsqcup_{J \in H-\operatorname{Spec}(A)} \operatorname{Spec}_{J}(A) .
$$

This partition is the so-called $H$-stratification of $\operatorname{Spec}(A)$.

When the $H$-spectrum of $A$ is finite this partition is a powerful tool in the study of the prime spectrum of $A$.

As we work in the generic case where $q$ is not a root of unity, the ring $R$ of $m \times n$ quantum matrices has a finite $\mathscr{H}=\left(\mathbb{K}^{*}\right)^{m+n}$-spectrum. Remarkably, for each $\mathscr{H}$-prime $J$, the $\operatorname{space} \operatorname{Spec}_{J}(R)$ is homeomorphic to $\operatorname{Spec}\left(\mathbb{K}\left[z_{1}^{ \pm 1}, \ldots, z_{d}^{ \pm 1}\right]\right)$ for some $d$ which depends on $J$. This $d$ is simply the (Krull) dimension of the $\mathscr{H}$-stratum $\operatorname{Spec}_{J}(R)$.

The work of Goodearl and Letzter spurred much research into the structure of $\operatorname{Spec}(R)$ in terms of the $\mathscr{H}$-spectrum. Some of the main themes in the study of the $\mathscr{H}$-spectrum have been computing its size, computing the structure of the poset of $\mathscr{H}$-primes under inclusion, and computing the dimensions of the $\mathscr{H}$-strata and how they are distributed. 


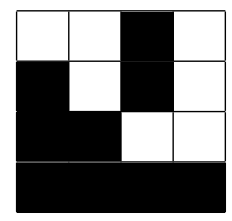

Figure 1. An example of a $4 \times 4$ Cauchon diagram.

The question of the size of the $\mathscr{H}$-spectrum of $R$ was answered in [Cauchon 2003b]. For many years the finiteness of the $\mathscr{H}$-spectrum of $R$ was known, but no formula for its size was known - except for small values of $m$ and $n-$ due to the complicated nature of the relations in $R$. Cauchon used his theory of deleting derivations to compute the size of the $\mathscr{H}$-spectrum of $R$. In particular, the set of $\mathscr{H}$ primes is in one-to-one correspondence with a set of combinatorial objects called Cauchon diagrams. In fact, Cauchon's method applies to a much broader class of algebras, the so-called CGL (Cauchon-Goodearl-Letzter) extensions, and the term "Cauchon diagram" has now acquired a more general meaning than the one we now describe for quantum matrices.

Definition. An $m \times n$ Cauchon diagram $C$ is simply an $m \times n$ grid consisting of $m n$ boxes in which certain boxes are coloured black. We require that if a box is black, then either every box strictly to its left is black or every box strictly above it is black. We let $\mathscr{C}_{m, n}$ denote the collection of $m \times n$ Cauchon diagrams.

Cauchon showed that the $\mathscr{H}$-primes of the ring $R$ of $m \times n$ quantum matrices are parameterized by the collection of $m \times n$ Cauchon diagrams, and he also gave a closed formula for the size of this set. Moreover it is known that the poset $\mathscr{H}$ $\operatorname{Spec}(R)$ (under inclusion) is isomorphic to a subposet of the symmetric group $S_{m+n}$ endowed with the Bruhat order [Launois 2007].

Some of the major questions that remain are to determine the possible dimensions of $\mathscr{H}$-strata that can occur in $R$ and to give a formula for the dimension of a stratum in terms of the associated Cauchon diagram. We answer these questions. In particular, we prove the following result.

Theorem 1.1. Let $m$ and $n$ be natural numbers. Then the dimensions of He-strata in $\mathcal{O}_{q}\left(M_{m, n}\right)$ are all at most $\min (m, n) ;$ moreover, for each $d \in\{0,1, \ldots, \min (m, n)\}$ there exists a d-dimensional He-stratum.

Previously, the best known bound for the dimensions of $\mathscr{H}$-strata in $O_{q}\left(M_{m, n}\right)$ was $m+n-1$, so this result represents a significant improvement.

Regarding the dimension of the stratum associated with a given Cauchon diagram, we give a formula which only relies on the Cauchon diagram; see Proposition 4.2. In fact, we are able to give a formula for a much broader class of algebras, 
called uniparameter CGL extensions. This class of algebras includes in particular the so-called quantum Schubert cells $U_{q}[w]$ defined by De Concini, Kac and Procesi (see Section 3C). The algebra $U_{q}[w]$ supports a rational torus action and the theory of Cauchon and Goodearl-Letzter can be applied to this algebra. The torus-invariant primes of this algebra have been studied recently and independently in [Cauchon and Mériaux 2009] and [Yakimov 2009]. As a consequence of our formula, we are able to give a formula for the dimension of the (0)-stratum of $U_{q}[w]$ which only depends on the Weyl group element $w$.

Regarding the $\mathscr{H}$-strata in $\mathrm{O}_{q}\left(M_{m, n}\right)$, we show even more. We say that an $m \times n$ Cauchon diagram $C$ contains another $m \times n$ Cauchon diagram $C^{\prime}$ if whenever a square is coloured black in $C^{\prime}$, the corresponding square is also coloured black in $C$. After additional investigations we are able to prove the following result.

Theorem 1.2. Let $P$ be an He-prime of $\mathrm{O}_{q}\left(M_{m, n}\right)$ whose associated He-stratum is $d$-dimensional. Then there exists a chain

$$
P=P_{0} \subsetneq P_{1} \subsetneq \cdots \subsetneq P_{d}
$$

of H-primes such that the dimension of the H-stratum associated to $P_{i}$ is $d-i$ and such that

$$
C_{0} \subsetneq C_{1} \subsetneq \cdots \subsetneq C_{d},
$$

where $C_{i}$ is the Cauchon diagram associated to $P_{i}$.

To prove this chain result, we need to understand the relation between inclusion of Cauchon diagrams and inclusion of the corresponding $H$-primes. One might naively expect these two posets to be isomorphic, but this is not the case. For instance, consider the algebra of $2 \times 2$ quantum matrices $O_{q}\left(M_{2}\right)$ generated by four indeterminates $Y_{1,1}, Y_{1,2}, Y_{2,1}, Y_{2,2}$, subject to the relations given in the beginning of this section. It is well known that the ideal $\left(Y_{1,1} Y_{2,2}-q Y_{1,2} Y_{2,1}\right)$ generated by the quantum determinant and $\left(Y_{2,1}, Y_{2,2}\right)$ are $\mathscr{H}$-invariant prime ideals in $\mathrm{O}_{q}\left(M_{2}\right)$. Clearly, $\left(Y_{1,1} Y_{2,2}-q Y_{1,2} Y_{2,1}\right) \subsetneq\left(Y_{2,1}, Y_{2,2}\right)$, but the corresponding Cauchon diagrams, which can be represented by the pictures in Figure 2, are not comparable.

Thus two $\mathscr{H}$-primes can be comparable (for the inclusion) and yet their corresponding Cauchon diagrams may fail to be comparable. Interestingly, if we consider things from the other direction, we see there is a definite relation between these two posets.
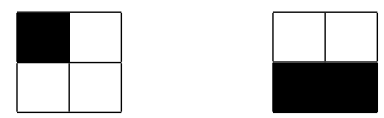

Figure 2. Cauchon diagrams representing the ideal generated by the quantum determinant and by $Y_{2,1}, Y_{2,2}$ respectively in $O_{q}\left(M_{2}\right)$. 
Theorem 1.3. If $C$ and $C^{\prime}$ are two $m \times n$ Cauchon diagrams with $C \subsetneq C^{\prime}$, then $J_{C} \subsetneq$ $J_{C^{\prime}}$, where $J_{C}$ and $J_{C^{\prime}}$ denote respectively the He-primes of $\mathrm{O}_{q}\left(M_{m, n}\right)$ associated to $C$ and $C^{\prime}$.

Again, we are able to prove Theorem 1.3 for a much broader class of algebras, called CGL extensions. This class of algebras contains quantum affine spaces, the algebra of $m \times n$ quantum matrices, positive parts of quantized enveloping algebras, and many other interesting families of algebras. These algebras support a rational action by a torus $H$ and have the property that they have only finitely many $H$-strata in the Goodearl-Letzter stratification.

The deleting derivations theory of Cauchon applies to CGL extensions and this gives new insights into the $H$-stratification in these cases. The $H$-primes are, just as in the case of quantum matrices, in one-to-one correspondence with combinatorial objects called Cauchon diagrams. These diagrams depend on the algebra, and a CGL extension other than quantum matrices has a different collection of Cauchon diagrams than the ones described earlier for $m \times n$ quantum matrices. Cauchon's original description [2003b] was just for quantum matrices, but other authors have since applied his deleting derivations theory to other classes of algebras. In [Launois et al. 2008] we gave a description of Cauchon diagrams for the quantum Grassmannian. Mériaux [2010] gave a description of these diagrams for the positive part of the quantized enveloping algebra of a simple Lie algebra, while Cauchon and Mériaux [2009] have recently described Cauchon diagrams in quantum Schubert cells. The set of Cauchon diagrams has a natural poset structure under inclusion; likewise the set of $H$-primes can be viewed as a poset under inclusion.

In this broader context, we are able to show the following result, of which Theorem 1.3 is a special case:

Theorem 1.4. If $w$ and $w^{\prime}$ are two Cauchon diagrams of a CGL extension $R$ with $w \subsetneq w^{\prime}$, then $J_{w} \subsetneq J_{w^{\prime}}$, where $J_{w}$ and $J_{w^{\prime}}$ denote respectively the (unique) $H$ primes associated to $w$ and $w^{\prime}$.

The outline of this paper is as follows. In Section 2 we give the necessary background on CGL extensions and we prove Theorem 1.4. In Section 3, we give a formula for the dimension of a stratum in a uniparameter CGL extension. Then we use this formula to compute the dimension of the (0)-stratum in a quantum Schubert cell. In Section 4 we describe the results obtained in the previous sections in the particular case of quantum matrices. Then we use these results in order to prove Theorems 1.1 and 1.2. In Section 5, after having seen the possible values that can occur as the dimension of an $\mathscr{H}$-stratum in quantum matrices, we give a conjecture about the number of $d$-dimensional $\mathscr{H}$-strata in $\mathcal{O}_{q}\left(M_{m, n}\right)$.

Throughout this paper, we use the following conventions. 
(i) If $I$ is a finite set, $|I|$ denotes its cardinality.

(ii) $\llbracket a, b \rrbracket:=\{i \in \mathbb{N} \mid a \leq i \leq b\}$.

(iii) $\mathbb{K}$ denotes a field and we set $\mathbb{K}^{*}:=\mathbb{K} \backslash\{0\}$.

(iv) If $A$ is a $\mathbb{K}$-algebra, then $\operatorname{Spec}(A)$ and $\operatorname{Prim}(A)$ denote respectively its prime and primitive spectra.

\section{2. $\boldsymbol{H}$-primes in CGL extensions}

In this section, we recall the notion of CGL extensions that was introduced in [Launois et al. 2006]. Examples include various quantum algebras in the generic case such as quantum affine spaces, quantum matrices, the positive part of quantized enveloping algebras of semisimple complex Lie algebras, etc. As we will see, the advantage of this class of algebras is that one can use both the stratification theory of Goodearl and Letzter and the theory of deleting derivations of Cauchon in order to study their prime and primitive spectra. This will allow us to investigate the topology of the $H$-spectrum of such algebras and prove Theorem 1.4.

\section{A. CGL extensions and the H-stratification theory of Goodearl and Letzter. In} this subsection, $N$ denotes a positive integer and $R$ is an iterated Ore extension; that is,

$$
R=\mathbb{K}\left[X_{1}\right]\left[X_{2} ; \sigma_{2}, \delta_{2}\right] \cdots\left[X_{N} ; \sigma_{N}, \delta_{N}\right],
$$

where $\sigma_{j}$ is an automorphism of the $\mathbb{K}$-algebra

$$
R_{j-1}:=\mathbb{K}\left[X_{1}\right]\left[X_{2} ; \sigma_{2}, \delta_{2}\right] \ldots\left[X_{j-1} ; \sigma_{j-1}, \delta_{j-1}\right]
$$

and $\delta_{j}$ is a $\mathbb{K}$-linear $\sigma_{j}$-derivation of $R_{j-1}$ for all $j \in \llbracket 2, N \rrbracket$. In other words, $R$ is a skew polynomial ring whose multiplication is defined by

$$
X_{j} a=\sigma_{j}(a) X_{j}+\delta_{j}(a)
$$

for all $j \in \llbracket 2, N \rrbracket$ and $a \in R_{j-1}$. Thus $R$ is a noetherian domain. Henceforth, we assume that $R$ is a $C G L$ extension:

Definition [Launois et al. 2006]. An iterated Ore extension $R$ is said to be a CGL extension if

(1) for all $j \in \llbracket 2, N \rrbracket, \delta_{j}$ is locally nilpotent;

(2) for all $j \in \llbracket 2, N \rrbracket$, there exists $q_{j} \in \mathbb{K}^{*}$ such that $\sigma_{j} \circ \delta_{j}=q_{j} \delta_{j} \circ \sigma_{j}$ and, for all $i \in \llbracket 1, j-1 \rrbracket$, there exists $\lambda_{j, i} \in \mathbb{K}^{*}$ such that $\sigma_{j}\left(X_{i}\right)=\lambda_{j, i} X_{i}$;

(3) none of the $q_{j}(2 \leq j \leq N)$ is a root of unity; and

(4) there exists a torus $H=\left(\mathbb{K}^{*}\right)^{d}$ that acts rationally by $\mathbb{K}$-automorphisms on $R$ such that 
- $X_{1}, \ldots, X_{N}$ are $H$-eigenvectors,

- the set $\left\{\lambda \in \mathbb{K}^{*} \mid(\exists h \in H)\left(h \cdot X_{1}=\lambda X_{1}\right)\right\}$ is infinite, and

- for all $j \in \llbracket 2, N \rrbracket$, there exists $h_{j} \in H$ such that $h_{j} \cdot X_{i}=\lambda_{j, i} X_{i}$ if $1 \leq i<j$ and $h_{j} \cdot X_{j}=q_{j} X_{j}$.

It follows from [Goodearl and Letzter 2000] that every $H$-prime ideal of $R$ is completely prime, so $H$-Spec $(R)$ coincides with the set of $H$-invariant completely prime ideals of $R$. Moreover there are at most $2^{N} H$-prime ideals in $R$. As a corollary, the $H$-stratification (1.3) breaks down the prime spectrum of $R$ into a finite number of parts, the $H$-strata. The geometric nature of the $H$-strata is well known: each $H$-stratum is homeomorphic to the scheme of irreducible subvarieties of a $\mathbb{K}$-torus [Brown and Goodearl 2002, Theorems II.2.13 and II.6.4]. However, the dimensions of these schemes are unknown in general.

2B. Quantum affine spaces. Let $N$ be a positive integer and let $\Lambda=\left(\Lambda_{i, j}\right) \in$ $M_{N}\left(\mathbb{K}^{*}\right)$ be a multiplicatively antisymmetric matrix; that is, $\Lambda_{i, j} \Lambda_{j, i}=\Lambda_{i, i}=1$ for all $i, j \in \llbracket 1, N \rrbracket$. The quantum affine space associated to $\Lambda$ is denoted by $\mathrm{O}_{\Lambda}\left(\mathbb{K}^{N}\right)=\mathbb{K}_{\Lambda}\left[T_{1}, \ldots, T_{N}\right]$; this is the $\mathbb{K}$-algebra generated by $N$ indeterminates $T_{1}, \ldots, T_{N}$ subject to the relations $T_{j} T_{i}=\Lambda_{j, i} T_{i} T_{j}$ for all $i, j \in \llbracket 1, N \rrbracket$. It is well known that $\mathrm{O}_{\Lambda}\left(\mathbb{K}^{N}\right)$ is an iterated Ore extension:

$$
\mathcal{O}_{\Lambda}\left(\mathbb{K}^{N}\right)=\mathbb{K}\left[T_{1}\right]\left[T_{2} ; \sigma_{2}\right] \cdots\left[T_{N} ; \sigma_{N}\right],
$$

where $\sigma_{j}$ is the automorphism defined by $\sigma_{j}\left(T_{i}\right)=\Lambda_{j, i} T_{i}$ for all $1 \leq i<j \leq N$. Observe that the torus $H=\left(\mathbb{K}^{*}\right)^{N}$ acts by automorphisms on $\mathbb{O}_{\Lambda}\left(\mathbb{K}^{N}\right)$ via:

$$
\left(a_{1}, \ldots, a_{N}\right) \cdot T_{i}=a_{i} T_{i} \text { for all } i \in \llbracket 1, N \rrbracket \text { and }\left(a_{1}, \ldots, a_{N}\right) \in H .
$$

Moreover, it is well known (see for instance [Launois et al. 2006, Corollary 3.8]) that $O_{\Lambda}\left(\mathbb{K}^{N}\right)$ is a CGL extension with this action of $H$. Hence $O_{\Lambda}\left(\mathbb{K}^{N}\right)$ has at most $2^{N} H$-prime ideals and they are all completely prime.

The $H$-stratification of $\operatorname{Spec}\left(O_{\Lambda}\left(\mathbb{K}^{N}\right)\right)$ was entirely described in [Brown and Goodearl 1996] when the group $\left\langle\Lambda_{i, j}\right\rangle$ is torsion-free and in [Goodearl and Letzter $1998]$ in the general case. We now recall these results.

Let $W$ denote the set of subsets of $\llbracket 1, N \rrbracket$. If $w \in W$, then we denote by $K_{w}$ the (two-sided) ideal of $O_{\Lambda}\left(\mathbb{K}^{N}\right)$ generated by the indeterminates $T_{i}$ with $i \in w$. It is easy to check that $K_{w}$ is an $H$-invariant completely prime ideal of $\mathrm{O}_{\Lambda}\left(\mathbb{K}^{N}\right)$.

Proposition 2.1 [Goodearl and Letzter 1998, Proposition 2.11].

(1) The ideals $K_{w}$ with $w \in W$ are exactly the $H$-prime ideals of $\mathscr{O}_{\Lambda}\left(\mathbb{K}^{N}\right)$. Hence there are exactly $2^{N} H$-prime ideals in this case.

(2) For all $w \in W$, the $H$-stratum associated to $K_{w}$ is given by $\operatorname{Spec}_{K_{w}}\left(\mathbb{O}_{\Lambda}\left(\mathbb{K}^{N}\right)\right)=\left\{P \in \operatorname{Spec}\left(\mathcal{O}_{\Lambda}\left(\mathbb{K}^{N}\right)\right) \mid P \cap\left\{T_{i} \mid i \in \llbracket 1, N \rrbracket\right\}=\left\{T_{i} \mid i \in w\right\}\right\}$. 
2C. The canonical partition of $\operatorname{Spec}(\boldsymbol{R})$. Let $R$ denote a CGL extension, as in Section 2A. Following [Cauchon 2003a], we construct the canonical partition of $\operatorname{Spec}(R)$, which gives new insight into the $H$-stratification of $\operatorname{Spec}(R)$. All references in this subsection, through page 184, are to the article just mentioned.

To describe the prime spectrum of $R$, Cauchon introduced the deleting derivations algorithm (Section 3.2). This algorithm constructs, for each $j \in \llbracket N+1,2 \rrbracket$, a family $\left\{X_{1}^{(j)}, \ldots, X_{N}^{(j)}\right\}$ of elements of the division ring of $\operatorname{fractions} \operatorname{Fract}(R)$ of $R$ defined as follows:

(1) When $j=N+1$, we set $\left(X_{1}^{(N+1)}, \ldots, X_{N}^{(N+1)}\right)=\left(X_{1}, \ldots, X_{N}\right)$.

(2) Assume that $j<N+1$ and that the $X_{i}^{(j+1)}(i \in \llbracket 1, N \rrbracket)$ are already constructed. Then it follows from Cauchon's Théorème 3.2.1 that $X_{j}^{(j+1)} \neq 0$ and that, for each $i \in \llbracket 1, N \rrbracket$, we have

$$
X_{i}^{(j)}= \begin{cases}X_{i}^{(j+1)} & \text { if } i \geq j, \\ \sum_{k=0}^{+\infty} \frac{\left(1-q_{j}\right)^{-k}}{[k] !_{q_{j}}} \delta_{j}^{k} \circ \sigma_{j}^{-k}\left(X_{i}^{(j+1)}\right)\left(X_{j}^{(j+1)}\right)^{-k} & \text { if } i<j,\end{cases}
$$

where $[k] !_{q_{j}}=[0]_{q_{j}} \times \cdots \times[k]_{q_{j}}$ with $[0]_{q_{j}}=1$ and $[i]_{q_{j}}=1+q_{j}+\cdots+q_{j}^{i-1}$ when $i \geq 1$.

Fix $j \in \llbracket 2, N+1 \rrbracket$. We denote by $R^{(j)}$ the subalgebra of $\operatorname{Fract}(R)$ generated by the $X_{i}^{(j)}$ :

$$
R^{(j)}:=\mathbb{K}\left\langle X_{1}^{(j)}, \ldots, X_{N}^{(j)}\right\rangle .
$$

The following results are Cauchon's Théorème 3.2.1 and Lemme 4.2.1.

(1) $R^{(j)}$ is isomorphic to an iterated Ore extension of the form

$$
\mathbb{K}\left[Y_{1}\right] \ldots\left[Y_{j-1} ; \sigma_{j-1}, \delta_{j-1}\right]\left[Y_{j} ; \tau_{j}\right] \cdots\left[Y_{N} ; \tau_{N}\right]
$$

by an isomorphism that sends $X_{i}^{(j)}$ to $Y_{i}(1 \leq i \leq N)$, where $\tau_{j}, \ldots, \tau_{N}$ denote the $\mathbb{K}$-linear automorphisms such that $\tau_{l}\left(Y_{i}\right)=\lambda_{l, i} Y_{i}(1 \leq i \leq l)$.

(2) Assume that $j \neq N+1$ and set $S_{j}:=\left\{\left(X_{j}^{(j+1)}\right)^{n}: n \in \mathbb{N}\right\}=\left\{\left(X_{j}^{(j)}\right)^{n}: n \in \mathbb{N}\right\}$. This is a multiplicative system of regular elements of $R^{(j)}$ and $R^{(j+1)}$, that satisfies the Ore condition in $R^{(j)}$ and $R^{(j+1)}$. Moreover

$$
R^{(j)} S_{j}^{-1}=R^{(j+1)} S_{j}^{-1} .
$$

It follows from these results that $R^{(j)}$ is a noetherian domain.

Notation. We set $\bar{R}:=R^{(2)}$ and $T_{i}:=X_{i}^{(2)}$ for all $i \in \llbracket 1, N \rrbracket$.

It follows from Cauchon's Proposition 3.2.1 that $\bar{R}$ is a quantum affine space in the indeterminates $T_{1}, \ldots, T_{N}$; hence his expression "effacement des dérivations". 
More precisely, let $\Lambda=\left(\mu_{i, j}\right) \in M_{N}\left(\mathbb{K}^{*}\right)$ be the multiplicatively antisymmetric matrix whose entries are defined by

$$
\mu_{j, i}= \begin{cases}\lambda_{j, i} & \text { if } i<j \\ 1 & \text { if } i=j \\ \lambda_{i, j}^{-1} & \text { if } i>j\end{cases}
$$

where the $\lambda_{j, i}$ with $i<j$ come from the CGL extension structure of $R$ (see page 180 for the definition). Then

$$
\bar{R}=\mathbb{K}_{\Lambda}\left[T_{1}, \ldots, T_{N}\right]=\mathbb{O}_{\Lambda}\left(\mathbb{K}^{N}\right) .
$$

The deleting derivations algorithm is used to relate the prime spectrum of a CGL extension $R$ to the prime spectrum of the associated quantum affine space $\bar{R}$. More precisely, Cauchon used this algorithm to construct embeddings

$$
\varphi_{j}: \operatorname{Spec}\left(R^{(j+1)}\right) \longrightarrow \operatorname{Spec}\left(R^{(j)}\right) \quad \text { for } j \in \llbracket 2, N \rrbracket,
$$

as follows (Section 4.3; see especially his Lemme 4.3.2).

Let $P \in \operatorname{Spec}\left(R^{(j+1)}\right)$. Then

$$
\varphi_{j}(P)= \begin{cases}P S_{j}^{-1} \cap R^{(j)} & \text { if } X_{j}^{(j+1)} \notin P, \\ g_{j}^{-1}\left(P /\left(X_{j}^{(j+1)}\right)\right) & \text { if } X_{j}^{(j+1)} \in P,\end{cases}
$$

where $g_{j}$ denotes the surjective homomorphism

$$
g_{j}: R^{(j)} \rightarrow R^{(j+1)} /\left(X_{j}^{(j+1)}\right)
$$

defined by

$$
g_{j}\left(X_{i}^{(j)}\right):=X_{i}^{(j+1)}+\left(X_{j}^{(j+1)}\right) .
$$

Cauchon's Proposition 4.3.1 states that $\varphi_{j}$ induces an increasing homeomorphism from the topological space

$$
\left\{P \in \operatorname{Spec}\left(R^{(j+1)}\right) \mid X_{j}^{(j+1)} \notin P\right\} \quad \text { onto } \quad\left\{Q \in \operatorname{Spec}\left(R^{(j)}\right) \mid X_{j}^{(j)} \notin Q\right\},
$$

whose inverse is also an increasing homeomorphism; also, $\varphi_{j}$ induces an increasing homeomorphism from

$$
\left\{P \in \operatorname{Spec}\left(R^{(j+1)}\right) \mid X_{j}^{(j+1)} \in P\right\}
$$

onto its image by $\varphi_{j}$, whose inverse similarly is an increasing homeomorphism. Note however that, in general, $\varphi_{j}$ is not an homeomorphism from $\operatorname{Spec}\left(R^{(j+1)}\right)$ onto its image.

Composing these embeddings, we get an embedding

$$
\varphi:=\varphi_{2} \circ \cdots \circ \varphi_{N}: \operatorname{Spec}(R) \longrightarrow \operatorname{Spec}(\bar{R}),
$$


which is called the canonical embedding from $\operatorname{Spec}(R)$ into $\operatorname{Spec}(\bar{R})$. This canonical embedding allows the construction of a partition of $\operatorname{Spec}(R)$ as follows.

We keep the notation of the previous sections. In particular, $W$ still denotes the set of all subsets of $\llbracket 1, N \rrbracket$. If $w \in W$, then we set

$$
\operatorname{Spec}_{w}(R):=\varphi^{-1}\left(\operatorname{Spec}_{K_{w}}(\bar{R})\right) .
$$

Moreover, we denote by $W^{\prime}$ the set of those $w \in W$ such that $\operatorname{Spec}_{w}(R) \neq \varnothing$. The elements of $W^{\prime}$ are called the Cauchon diagrams of the CGL extension $R$. It follows from Cauchon's Proposition 4.4.1 that

$$
\operatorname{Spec}(R)=\bigsqcup_{w \in W^{\prime}} \operatorname{Spec}_{w}(R) \text { and }\left|W^{\prime}\right| \leq|W|=2^{N} .
$$

This partition is called the canonical partition of $\operatorname{Spec}(R)$, and it gives another way to understand the $H$-stratification, since, by Cauchon's Théorème 5.5.2, these two partitions coincide. As a consequence, we obtain another description of the $H$-prime ideals of $R$ :

Proposition 2.2 [Cauchon 2003a, Lemme 5.5.8 and Théorème 5.5.2].

(1) Let $w \in W^{\prime}$. There exists a (unique) $H$-invariant (completely) prime ideal $J_{w}$ of $R$ such that $\varphi\left(J_{w}\right)=K_{w}$, where $K_{w}$ denotes the ideal of $\bar{R}$ generated by the $T_{i}$ with $i \in w$.

(2) $H-\operatorname{Spec}(R)=\left\{J_{w} \mid w \in W^{\prime}\right\}$.

(3) $\operatorname{Spec}_{J_{w}}(R)=\operatorname{Spec}_{w}(R)$ for all $w \in W^{\prime}$.

2D. The map $w \mapsto J_{w}$ is increasing. In this section, we prove Theorem 1.4.

Theorem 2.3. Let $R$ be a CGL extension and let $w, w^{\prime} \in W^{\prime}$ be two Cauchon diagrams of $R$. If $w \subseteq w^{\prime}$ then $J_{w} \subseteq J_{w^{\prime}}$.

Proof. For $j \in \llbracket 2, N+1 \rrbracket$ and $P \in \operatorname{Spec}(R)$, we set $P^{(j)}:=\varphi_{j} \circ \cdots \circ \varphi_{N}(P)$.

We prove by induction on $j$ that

$$
J_{w}^{(j)} \subseteq J_{w^{\prime}}^{(j)} \quad \text { for } j \in \llbracket 2, N+1 \rrbracket .
$$

When $j=2$, we have $J_{w}^{(2)}=K_{w}$ and $J_{w^{\prime}}^{(2)}=K_{w^{\prime}}$. As $w \subseteq w^{\prime}$, we have $K_{w} \subseteq K_{w^{\prime}}$, so $J_{w}^{(2)} \subseteq J_{w^{\prime}}^{(2)}$, as desired.

We assume $j \leq N$ and $J_{w}^{(j)} \subseteq J_{w^{\prime}}^{(j)}$. We need to prove that $J_{w}^{(j+1)} \subseteq J_{w^{\prime}}^{(j+1)}$. Observe first that if $X_{j}^{(j)} \notin J_{w^{\prime}}^{(j)}$, then

$$
J_{w}^{(j)}, J_{w^{\prime}}^{(j)} \in\left\{Q \in \operatorname{Spec}\left(R^{(j)}\right) \mid X_{j}^{(j)} \notin Q\right\} .
$$

As $\varphi_{j}$ induces an increasing homeomorphism, still denoted $\varphi_{j}$, from

$$
\left\{P \in \operatorname{Spec}\left(R^{(j+1)}\right) \mid X_{j}^{(j+1)} \notin P\right\}
$$


onto

$$
\left\{Q \in \operatorname{Spec}\left(R^{(j)}\right) \mid X_{j}^{(j)} \notin Q\right\},
$$

whose inverse is also an increasing homeomorphism, we obtain

$$
J_{w}^{(j+1)}=\varphi_{j}^{-1}\left(J_{w}^{(j)}\right) \subseteq \varphi_{j}^{-1}\left(J_{w^{\prime}}^{(j)}\right)=J_{w^{\prime}}^{(j+1)},
$$

as desired.

Similarly, if $X_{j}^{(j)} \in J_{w}^{(j)}$, then both $J_{w}^{(j)}$ and $J_{w^{\prime}}^{(j)}$ belong to

$$
\varphi_{j}\left(\left\{P \in \operatorname{Spec}\left(R^{(j+1)}\right) \mid X_{j}^{(j+1)} \in P\right\}\right) .
$$

As $\varphi_{j}$ induces an increasing homeomorphism, still denoted $\varphi_{j}$, from

$$
\left\{P \in \operatorname{Spec}\left(R^{(j+1)}\right) \mid X_{j}^{(j+1)} \in P\right\}
$$

onto its image whose inverse is also an increasing homeomorphism, we get

$$
J_{w}^{(j+1)}=\varphi_{j}^{-1}\left(J_{w}^{(j)}\right) \subseteq \varphi_{j}^{-1}\left(J_{w^{\prime}}^{(j)}\right)=J_{w^{\prime}}^{(j+1)},
$$

as desired.

It only remains to deal with the case where $X_{j}^{(j)} \in J_{w^{\prime}}^{(j)}$ and $X_{j}^{(j)} \notin J_{w}^{(j)}$, so $J_{w}^{(j)}=J_{w}^{(j+1)} S_{j}^{-1} \cap R^{(j)}, \quad J_{w}^{(j+1)}=J_{w}^{(j)} S_{j}^{-1} \cap R^{(j+1)}, \quad J_{w^{\prime}}^{(j)}=g_{j}^{-1}\left(J_{w^{\prime}}^{(j+1)} /\left(X_{j}^{(j+1)}\right)\right)$.

In order to simplify the notation we set

$$
Y_{i}:=X_{i}^{(j)} \text { and } Z_{i}:=X_{i}^{(j+1)} .
$$

Also we let $A$ denote the subalgebra of $R^{(j+1)}$ generated by the $Z_{i}$ with $i \neq j$ :

$$
A:=\mathbb{K}\left\langle Z_{1}, \ldots, Z_{j-1}, Z_{j+1}, \ldots, Z_{N}\right\rangle .
$$

Observe that $R^{(j+1)}=A\left[Z_{j} ; \sigma, \delta\right]$, where $\sigma$ denotes the automorphism of $A$ defined by $\sigma\left(Z_{i}\right)=\lambda_{j, i} Z_{i}=\sigma_{j}\left(Z_{i}\right)$ if $i<j$ and $\sigma\left(Z_{i}\right)=\lambda_{i, j}^{-1} Z_{i}$ otherwise; and where $\delta$ denotes the $\sigma$-derivation of $A$ defined by $\delta\left(Z_{i}\right)=\delta_{j}\left(Z_{i}\right)$ if $i<j$ and $\delta\left(Z_{i}\right)=0$ otherwise. One can easily check that this Ore extension satisfies the conditions of [Cauchon 2003a, Section 2], so the map $\theta: A \rightarrow R^{(j+1)} S_{j}^{-1}=R^{(j)} S_{j}^{-1}$ defined by

$$
\theta(a)=\sum_{k=0}^{+\infty} \frac{\left(1-q_{j}\right)^{-k}}{[k] !_{q_{j}}} \delta^{k} \circ \sigma^{-k}(a) Z_{j}^{-k} \text { for } a \in A
$$

is an homomorphism. Observe that by the definition of the deleting derivations algorithm we have $\theta\left(Z_{i}\right)=Y_{i}$ for all $i \neq j$. Hence $\theta(A) \subset R^{(j)}$ and $R^{(j)}$ is the subalgebra of $R^{(j+1)} S_{j}^{-1}$ generated by $\theta(A)$ and $Z_{j}=Y_{j}$.

Again to simplify the notation, we set

$$
P:=J_{w}^{(j+1)}, \quad P^{\prime}:=J_{w}^{(j)}, \quad Q=J_{w^{\prime}}^{(j+1)}, \quad Q^{\prime}:=J_{w^{\prime}}^{(j)} .
$$


Let $z \in P$ with $z \neq 0$. We need to prove that $z \in Q$. First we can write

$$
z=\sum_{t=0}^{d} a_{t} Z_{j}^{t}
$$

where $a_{t} \in A$ and $a_{d} \neq 0$; moreover, this expression for $z$ in this form is unique. If $a_{0}=0$, then $z \in\left(Z_{j}\right) \subseteq Q$. So we assume that $a_{0} \neq 0$.

For every $t$, there exists $k_{t}$ minimal such that $\delta^{k_{t}+1}\left(a_{t}\right)=0$ (recall that $\delta$ is locally nilpotent), so

$$
\theta\left(a_{t}\right)=\sum_{k=0}^{k_{t}} \frac{\left(1-q_{j}\right)^{-k}}{[k] !_{q_{j}}} \delta^{k} \circ \sigma^{-k}\left(a_{t}\right) Z_{j}^{-k}
$$

By induction on the degree of local nilpotency we get:

$$
a_{t}=\theta\left(a_{t}\right)+\sum_{k=1}^{k_{t}} \mu_{k, t} \theta\left(\delta^{k} \circ \sigma^{-k}\left(a_{t}\right)\right) Z_{j}^{-k}=\sum_{k=0}^{k_{t}} \mu_{k, t} \theta\left(\delta^{k} \circ \sigma^{-k}\left(a_{t}\right)\right) Z_{j}^{-k},
$$

where $\mu_{0, t}=1$ and $\mu_{k, t} \in \mathbb{K}$. Let $m$ be the maximum of the $k_{t}-t$. Then

$$
z Z_{j}^{m}=\sum_{t=0}^{d} a_{t} Z_{j}^{t+m}=\sum_{t=0}^{d} \sum_{k=0}^{k_{t}} \mu_{k, t} \theta\left(\delta^{k} \circ \sigma^{-k}\left(a_{t}\right)\right) Z_{j}^{t+m-k} \in R^{(j)} .
$$

Thus $z Z_{j}^{m} \in R^{(j)} \cap P S_{j}^{-1}=P^{\prime}$. Hence

$$
z Z_{j}^{m}=\sum_{t=0}^{d} \sum_{k=0}^{k_{t}} \mu_{k, t} \theta\left(\delta^{k} \circ \sigma^{-k}\left(a_{t}\right)\right) Z_{j}^{t+m-k} \in P^{\prime} .
$$

We let $A^{\prime}$ denote the subalgebra of $R^{(j)}$ generated by $Y_{i}$ with $i \neq j$, or equivalently, the image of $\theta$. As $P^{\prime}$ is an $H$-prime ideal of

$$
R^{(j)}=A^{\prime}\left[Z_{j} ; \sigma\right],
$$

it follows from [Launois et al. 2006, Corollary 2.4] that the coefficient of $Z_{j}^{l}$ in the previous sum belongs to $P^{\prime}$ for every nonnegative integer $l$. In particular, the coefficient of degree $m$ is in $P^{\prime}$. Hence, by setting $k=t$ in (2.11), we obtain

$$
\sum_{t=0}^{d} \mu_{t, t} \theta\left(\delta^{t} \circ \sigma^{-t}\left(a_{t}\right)\right) \in P^{\prime} \subseteq Q^{\prime}
$$

As $Q^{\prime}=g_{j}^{-1}\left(Q /\left(Z_{j}\right)\right)$ and $\left(Z_{j}\right) \subseteq Q$, we get that

$$
\sum_{t=0}^{d} \mu_{t, t} \delta^{t} \circ \sigma^{-t}\left(a_{t}\right) \in Q .
$$


Since $Z_{j} \in Q$, we see that $\delta(a)=Z_{j} a-\sigma(a) Z_{j} \in Q$ for every $a \in A$. Hence we deduce from (2.12) that $a_{0}=\mu_{0,0} a_{0} \in Q$. Since $\left(Z_{j}\right) \subseteq Q$, we obtain, as desired,

$$
z=a_{0}+\left(\sum_{t=1}^{d} a_{t} Z_{j}^{t-1}\right) Z_{j} \in Q .
$$

\section{Dimension of $\boldsymbol{H}$-strata of uniparameter CGL extensions}

In this section, we obtain a formula for the dimension of a stratum of a uniparameter CGL extension, and apply it to compute the dimension of the (0)-stratum of a quantum Schubert cell.

3A. Uniparameter CGL extensions. In this section, we assume that $R$ is a uniparameter CGL extension, that is, $R$ is a CGL extension such that there exist an antisymmetric matrix $\left(a_{i, j}\right) \in \mathcal{M}_{N}(\mathbb{Z})$ and $q \in \mathbb{R}^{*}$ not a root of unity such that $\lambda_{j, i}=q^{a_{j, i}}$ for all $1 \leq i<j \leq N$.

3B. Dimension of $\boldsymbol{H}$-strata of uniparameter $\boldsymbol{C G L}$ extensions. The aim of this section is to give a formula for the dimension of the $H$-stratum in $R$ associated to a Cauchon diagram $w \in W^{\prime}$. We need to introduce the following definition.

Definition. Let $w \in W^{\prime}$ be a Cauchon diagram of $R$. Let $\left\{l_{1}<\cdots<l_{d}\right\}:=$ $\llbracket 1, N \rrbracket \backslash w$ be the complement of $w$. We define the skew-adjacency matrix, $M_{R}(w)$, of $w$ to be the $d \times d$ matrix whose $(i, j)$ entry is $a_{l_{i} l_{j}}$.

Theorem 3.1. Let $w \in W^{\prime}$. Then the $H$-stratum associated to $J_{w}$ is homeomorphic to the prime spectrum of a commutative Laurent polynomial ring over $\mathbb{R}$ in $\operatorname{dim}_{\mathbb{Q}}\left(\operatorname{ker}\left(M_{R}(w)\right)\right)$ indeterminates.

Proof. Let $w \in W^{\prime}$ be a Cauchon diagram of $R$. Let $\left\{l_{1}<\cdots<l_{d}\right\}:=\llbracket 1, N \rrbracket \backslash w$ be the complement of $w$. From [Cauchon 2003a, Théorèmes 5.1.1 and 5.5.1] we know that the canonical embedding induces an inclusion-preserving homeomorphism from the $H$-stratum $\operatorname{Spec}_{J_{w}}(R)$ of $R$ associated to $J_{w}$ onto the $H$-stratum $\operatorname{Spec}_{K_{w}}(\bar{R})$ of $\bar{R}$ associated to $K_{w}$. Hence we deduce from Proposition 2.1 that

$$
\begin{aligned}
\operatorname{Spec}_{J_{w}}(R) & \simeq \operatorname{Spec}_{K_{w}}(\bar{R}) \\
& \simeq\left\{P \in \operatorname{Spec}(\bar{R}) \mid P \cap\left\{T_{1}, \ldots, T_{N}\right\}=\left\{T_{i} \mid i \in w\right\}\right\} .
\end{aligned}
$$

Recall that $\bar{R}=\mathbb{K}_{\Lambda}\left[T_{1}, T_{2}, \ldots, T_{N}\right]$, where $\Lambda$ denotes the $N \times N$ matrix whose entries are defined by $\Lambda_{k, l}=q^{a_{k, l}}$ for all $k, l \in \llbracket 1, N \rrbracket$. Let $\Lambda_{w}$ denote the multiplicatively antisymmetric $d \times d$ matrix whose entries are defined by $\left(\Lambda_{w}\right)_{i, j}=$ $q^{M_{R}(w)_{i, j}}=q^{a_{l_{i} l_{j}}}$. 
As $K_{w}$ is the prime ideal generated by the indeterminates $T_{i}$ such that $i \in w$, the algebra $\bar{R} / K_{w}$ is isomorphic to the quantum affine space $\mathbb{K}_{\Lambda_{w}}\left[t_{1}, \ldots, t_{d}\right]$ by an isomorphism that sends $T_{l_{i}}+K_{w}$ to $t_{i}$ and $T_{k}$ to 0 if $k \neq l_{i}$.

To finish the proof, we use the same idea as in [Launois and Lenagan 2007, Corollary 1.3].

We denote by $P\left(\Lambda_{w}\right)$ the quantum torus associated to $\mathbb{K}_{\Lambda_{w}}\left[t_{1}, \ldots, t_{d}\right]$ :

$$
P\left(\Lambda_{w}\right):=\mathbb{K}_{\Lambda_{w}}\left[t_{1}, \ldots, t_{d}\right] \Sigma^{-1},
$$

where $\Sigma$ denotes the multiplicative system of $\mathbb{K}_{\Lambda_{w}}\left[t_{1}, \ldots, t_{d}\right]$ generated by the normal elements $t_{1}, \ldots, t_{d}$.

It follows from (3.13) that

$$
\operatorname{Spec}_{K_{w}}(\bar{R}) \simeq \operatorname{Spec}_{(0)}\left(\mathbb{K}_{\Lambda_{w}}\left[t_{1}, \ldots, t_{d}\right]\right) \simeq \operatorname{Spec}\left(P\left(\Lambda_{w}\right)\right) .
$$

Next, $\operatorname{Spec}\left(P\left(\Lambda_{w}\right)\right)$ is Zariski-homeomorphic via extension and contraction to the prime spectrum of the centre $Z\left(P\left(\Lambda_{w}\right)\right)$ of $P\left(\Lambda_{w}\right)$, by [Goodearl and Letzter 1998, Corollary 1.5]. Further, as we shall see, $Z\left(P\left(\Lambda_{w}\right)\right)$ is a Laurent polynomial ring. To make this result precise, we need to introduce the following notation.

If $\underline{s}=\left(s_{1}, \ldots, s_{d}\right) \in \mathbb{Z}^{d}$, then we set $t^{\underline{s}}:=t_{1}^{s_{1}} \ldots t_{d}^{s_{d}} \in P\left(\Lambda_{w}\right)$. As in [Goodearl and Letzter 1998], we denote by $\sigma: \mathbb{Z}^{d} \times \mathbb{Z}^{d} \rightarrow \mathbb{K}^{*}$ the antisymmetric bicharacter defined by

$$
\sigma(\underline{s}, \underline{t}):=\prod_{i, j=1}^{d}\left(\Lambda_{w}\right)_{i, j}^{s_{i} t_{j}}=q^{\sum_{i, j=1}^{d} a_{l_{i}, l_{j} s_{i} t_{j}}} \quad \text { for all } \underline{s}, \underline{t} \in \mathbb{Z}^{d} .
$$

Then it follows from [Goodearl and Letzter 1998, 1.3] that the centre $Z\left(P\left(\Lambda_{w}\right)\right)$ of $P\left(\Lambda_{w}\right)$ is a Laurent polynomial ring over $\mathbb{K}$ in the variables $\left(t \frac{b_{1}}{{ }^{ \pm 1}}, \ldots,\left(t \frac{b_{r}}{}\right)^{ \pm 1}\right.$, where $\left(\underline{b_{1}}, \ldots, \underline{b_{r}}\right)$ is any basis of

$$
V:=\left\{\underline{s} \in \mathbb{Z}^{d} \mid \sigma(\underline{s},-) \equiv 1\right\} .
$$

Since $q$ is not a root of unity, easy computations show that $\underline{s} \in V$ if and only if $M_{R}(w)^{t} \underline{s}^{t}=0$. Hence the centre $Z\left(P\left(\Lambda_{w}\right)\right)$ of $P\left(\Lambda_{w}\right)$ is a Laurent polynomial ring in $\operatorname{dim}_{\mathbb{Q}}\left(\operatorname{ker}\left(M_{R}(w)^{t}\right)\right)=\operatorname{dim}_{\mathbb{Q}}\left(\operatorname{ker}\left(M_{R}(w)\right)\right)$ indeterminates.

To summarize, we have

$$
\operatorname{Spec}_{J_{w}}(R) \simeq \operatorname{Spec}_{K_{w}}(\bar{R}) \simeq \operatorname{Spec}\left(P\left(\Lambda_{w}\right)\right) \simeq \operatorname{Spec}\left(Z\left(P\left(\Lambda_{w}\right)\right)\right),
$$

and $Z\left(P\left(\Lambda_{w}\right)\right)$ is a Laurent polynomial ring in $\operatorname{dim}_{\mathbb{Q}}\left(\operatorname{ker}\left(M_{R}(w)\right)\right)$ indeterminates, as desired.

3C. Application to quantum Schubert cells. We now use Theorem 3.1 to compute the dimension of the (0)-stratum of quantum Schubert cells. We first recall the definition of quantum Schubert cells. 
Let $\mathfrak{g}$ be a simple Lie $\mathbb{C}$-algebra of rank $n$. We denote by $\pi=\left\{\alpha_{1}, \ldots, \alpha_{n}\right\}$ the set of simple roots associated to a triangular decomposition $\mathfrak{g}=\mathfrak{n}^{-} \oplus \mathfrak{h} \oplus \mathfrak{n}^{+}$. Recall that $\pi$ is a basis of a Euclidean vector space $E$ over $\mathbb{R}$, whose inner product is denoted by $($,$) ( E$ is usually denoted by $\mathfrak{h}_{\mathbb{R}}^{*}$ in Bourbaki.) We denote by $W$ the Weyl group of $\mathfrak{g}$, that is, the subgroup of the orthogonal group of $E$ generated by the reflections $s_{i}:=s_{\alpha_{i}}$, for $i \in\{1, \ldots, n\}$, with reflecting hyperplanes $H_{i}:=\left\{\beta \in E \mid\left(\beta, \alpha_{i}\right)=0\right\}$, $i \in\{1, \ldots, n\}$. The length of $w \in W$ is denoted by $l(w)$. Further, we denote by $w_{0}$ the longest element of $W$. Finally, we denote by $A=\left(a_{i j}\right) \in M_{n}(\mathbb{Z})$ the Cartan matrix associated to these data. As $\mathfrak{g}$ is simple, $a_{i j} \in\{0,-1,-2,-3\}$ for all $i \neq j$.

Recall that the scalar product of two roots $(\alpha, \beta)$ is always an integer. We assume that the short roots have length $\sqrt{2}$.

For all $i \in\{1, \ldots, n\}$, set $q_{i}:=q^{\left(\alpha_{i}, \alpha_{i}\right) / 2}$ and

$$
\left[\begin{array}{c}
m \\
k
\end{array}\right]_{i}:=\frac{\left(q_{i}-q_{i}^{-1}\right) \ldots\left(q_{i}^{m-1}-q_{i}^{1-m}\right)\left(q_{i}^{m}-q_{i}^{-m}\right)}{\left(q_{i}-q_{i}^{-1}\right) \ldots\left(q_{i}^{k}-q_{i}^{-k}\right)\left(q_{i}-q_{i}^{-1}\right) \ldots\left(q_{i}^{m-k}-q_{i}^{k-m}\right)}
$$

for all integers $0 \leq k \leq m$. By convention,

$$
\left[\begin{array}{c}
m \\
0
\end{array}\right]_{i}:=1
$$

The quantised enveloping algebra $U_{q}(\mathfrak{g})$ of $\mathfrak{g}$ over $\mathbb{C}$ associated to the previous data is the $\mathbb{K}$-algebra generated by the indeterminates $E_{1}, \ldots, E_{n}, F_{1}, \ldots, F_{n}$, $K_{1}^{ \pm 1}, \ldots, K_{n}^{ \pm 1}$ subject to the following relations:

$$
\begin{gathered}
K_{i} K_{j}=K_{j} K_{i}, \\
K_{i} E_{j} K_{i}^{-1}=q_{i}^{a_{i j}} E_{j} \text { and } K_{i} F_{j} K_{i}^{-1}=q_{i}^{-a_{i j}} F_{j}, \\
E_{i} F_{j}-F_{j} E_{i}=\delta_{i j} \frac{K_{i}-K_{i}^{-1}}{q_{i}-q_{i}^{-1}},
\end{gathered}
$$

and the quantum Serre relations:

$$
\sum_{k=0}^{1-a_{i j}}(-1)^{k}\left[\begin{array}{c}
1-a_{i j} \\
k
\end{array}\right]_{i} E_{i}^{1-a_{i j}-k} E_{j} E_{i}^{k}=0(i \neq j),
$$

and

$$
\sum_{k=0}^{1-a_{i j}}(-1)^{k}\left[\begin{array}{c}
1-a_{i j} \\
k
\end{array}\right]_{i} F_{i}^{1-a_{i j}-k} F_{j} F_{i}^{k}=0(i \neq j) .
$$

We refer the reader to [Brown and Goodearl 2002; Jantzen 1996; Joseph 1995] for more details on this (Hopf) algebra. Further, as usual, we denote by $U_{q}^{+}(\mathfrak{g})$ (resp. $\left.U_{q}^{-}(\mathfrak{g})\right)$ the subalgebra of $U_{q}(\mathfrak{g})$ generated by $E_{1}, \ldots, E_{n}\left(\operatorname{resp} . F_{1}, \ldots, F_{n}\right)$ and by $U^{0}$ the subalgebra of $U_{q}(\mathfrak{g})$ generated by $K_{1}^{ \pm 1}, \ldots, K_{n}^{ \pm 1}$. 
To each reduced decomposition of the longest element $w_{0}$ of the Weyl group $W$ of $\mathfrak{g}$, Lusztig has associated a PBW basis of $U_{q}^{+}(\mathfrak{g})$; see, for instance, [Lusztig 1993, Chapter 37; Jantzen 1996, Chapter 8; Brown and Goodearl 2002, I.6.7]. The construction relates to a braid group action by automorphisms on $U_{q}^{+}(\mathfrak{g})$. We use the convention of [Jantzen 1996, Chapter 8]. In particular, for any $\alpha \in \pi$, we define the braid automorphism $T_{\alpha}$ of the algebra $U_{q}(\mathfrak{g})$ as in [Jantzen 1996, p. 153]. We set $T_{i}:=T_{\alpha_{i}}$. It was proved by Lusztig that the automorphisms $T_{i}$ satisfy the braid relations, that is, if $s_{i} s_{j}$ has order $m$ in $W$, then

$$
T_{i} T_{j} T_{i} \ldots=T_{j} T_{i} T_{j} \ldots,
$$

where there are exactly $m$ factors on each side of this equality.

Consider any $w \in W$, and set $t:=l(w)$. Let $w=s_{i_{1}} \circ \cdots \circ s_{i_{t}}\left(i_{j} \in\{1, \ldots, n\}\right)$ be a reduced decomposition of $w$. It is well known that $\beta_{1}=\alpha_{i_{1}}, \beta_{2}=s_{i_{1}}\left(\alpha_{i_{2}}\right), \ldots$, $\beta_{t}=s_{i_{1}} \circ \cdots \circ s_{i_{t-1}}\left(\alpha_{i_{t}}\right)$ are distinct positive roots and that the set $\left\{\beta_{1}, \ldots, \beta_{t}\right\}$ does not depend on the chosen reduced expression of $w$. Similarly, we define elements $E_{\beta_{k}}$ of $U_{q}(\mathfrak{g})$ by

$$
E_{\beta_{k}}:=T_{i_{1}} \cdots T_{i_{k-1}}\left(E_{i_{k}}\right) .
$$

Note that the elements $E_{\beta_{k}}$ depend on the reduced decomposition of $w$. The following well-known results were proved by Lusztig and Levendorskii-Soibelman.

Theorem 3.2 [Levendorskiı̌ and Soibelman 1991].

(1) For all $k \in\{1, \ldots, t\}$, the element $E_{\beta_{k}}$ belongs to $U_{q}^{+}(\mathfrak{g})$.

(2) If $\beta_{k}=\alpha_{i}$, then $E_{\beta_{k}}=E_{i}$.

(3) For all $1 \leq i<j \leq t$, we have

$$
E_{\beta_{j}} E_{\beta_{i}}-q^{-\left(\beta_{i}, \beta_{j}\right)} E_{\beta_{i}} E_{\beta_{j}}=\sum a_{k_{i+1}, \ldots, k_{j-1}} E_{\beta_{i+1}}^{k_{i+1}} \cdots E_{\beta_{j-1}}^{k_{j-1}},
$$

where each $a_{k_{i+1}, \ldots, k_{j-1}}$ belongs to $\mathbb{K}$.

We denote by $U_{q}[w]$ the subalgebra of $U_{q}^{+}(\mathfrak{g})$ generated by $E_{\beta_{1}}, \ldots, E_{\beta_{t}}$. It is well known that $U_{q}[w]$ does not depend on the reduced decomposition of $w$. Moreover, the monomials $E_{\beta_{1}}^{k_{1}} \cdots E_{\beta_{t}}^{k_{t}}$, with $k_{1}, \ldots, k_{t} \in \mathbb{N}$, form a linear basis of $U_{q}[w]$. As a consequence of this result, $U_{q}[w]$ can be presented as a skewpolynomial algebra:

$$
U_{q}[w]=\mathbb{K}\left[E_{\beta_{1}}\right]\left[E_{\beta_{2}} ; \sigma_{2}, \delta_{2}\right] \cdots\left[E_{\beta_{t}} ; \sigma_{t}, \delta_{t}\right],
$$

where each $\sigma_{i}$ is a linear automorphism and each $\delta_{i}$ is a $\sigma_{i}$-derivation of the appropriate subalgebra. In particular, $U_{q}[w]$ is a noetherian domain and its group of invertible elements is reduced to nonzero elements of the base-field.

The torus $\mathscr{H}:=\left(\mathbb{K}^{*}\right)^{n}$ acts rationally by automorphisms on $U_{q}^{+}(\mathfrak{g})$ via

$$
\left(h_{1}, \ldots, h_{n}\right) \cdot E_{i}=h_{i} E_{i} \quad \text { for all } i \in\{1, \ldots, n\} .
$$


(It is easy to check that the quantum Serre relations are preserved by the group $\mathscr{H}$.) It is also well known that this action of $\mathscr{H}$ on $U_{q}^{+}(\mathfrak{g})$ restricts to a rational action of $\mathscr{H}$ on $U_{q}[w]$. Observe that $(0)$ is an $\mathscr{H}$-prime in $U_{q}[w]$ as this algebra is a domain.

It was proved by Cauchon [2003a, Proposition 6.1.2 and Lemme 6.2.1] that $U_{q}[w]$ is a uniparameter CGL extension with the following associated antisymmetric matrix:

$$
\left(\begin{array}{ccccc}
0 & \left(\beta_{1}, \beta_{2}\right) & \ldots & \ldots & \left(\beta_{1}, \beta_{t}\right) \\
-\left(\beta_{1}, \beta_{2}\right) & 0 & \left(\beta_{2}, \beta_{3}\right) & & \left(\beta_{2}, \beta_{t}\right) \\
\vdots & \ddots & \ddots & \ddots & \vdots \\
\vdots & & \ddots & 0 & \left(\beta_{t-1}, \beta_{t}\right) \\
-\left(\beta_{1}, \beta_{t}\right) & \ldots & \ldots & -\left(\beta_{t-1}, \beta_{t}\right) & 0
\end{array}\right) .
$$

The kernel of this matrix has been described by De Concini and Procesi [1993, Lemma 10.4 and 10.6] who proved that the kernel of this matrix identifies with $\operatorname{ker}\left(\operatorname{id}_{E}+w\right)$. So we deduce from Theorem 3.1 the following result.

Proposition 3.3. The dimension of the stratum associated to (0) in $U_{q}[w]$ is

$$
\operatorname{dim} \operatorname{ker}\left(\operatorname{id}_{E}+w\right) \text {. }
$$

It follows from [Cauchon and Mériaux 2009, Proposition 2.2.1] that the algebra $\mathcal{O}_{q}\left(M_{m, n}\right)$ of quantum matrices can be presented as a quantum Schubert cell. So one can use the previous proposition in order to retrieve the dimension of the (0)stratum of $\mathscr{O}_{q}\left(M_{m, n}\right)$ that was first obtained in [Launois and Lenagan 2007].

\section{Quantum matrices}

In this section we investigate in more detail the dimensions of the strata occurring in the Goodearl-Letzter stratification of the ring $R=\mathrm{O}_{q}\left(M_{m, n}\right)$ of $m \times n$ quantum matrices, and we prove Theorems 1.1 and 1.2. Throughout this section, $q$ denotes a nonzero element of $\mathbb{K}$ that is not a root of unity. The ring $R$ is known to be a quantum Schubert cell by [Cauchon and Mériaux 2009, Proposition 2.2.1], so we know (see the previous section) that $\mathrm{O}_{q}\left(M_{m, n}\right)$ is a uniparameter CGL extension. Nevertheless we start by explicitly describing the results of the previous sections in this situation.

4A. Quantum matrices as a CGL extension. This section serves to show that quantum matrix rings give examples of CGL extensions and that we can therefore draw upon the background given in Section 2.

It is well known that $R=\mathcal{O}_{q}\left(M_{m, n}\right)$ can be presented as an iterated Ore extension over $\mathbb{K}$ with the generators $Y_{i, \alpha}$ given in the beginning of Section 1 adjoined in 
lexicographic order. Thus the ring $R$ is a noetherian domain; we denote by $F$ its skew-field of fractions. Since $q$ is not a root of unity, it follows from [Goodearl and Letzter 1994, Theorem 3.2] that all prime ideals of $R$ are completely prime.

The algebras $\mathscr{O}_{q}\left(M_{m, n}\right)$ and $\mathscr{O}_{q}\left(M_{n, m}\right)$ are isomorphic. Because of this, we can and will assume that $n \leq m$.

$\mathscr{H}:=\left(\mathbb{K}^{*}\right)^{m+n}$ acts on $R$ by $\mathbb{K}$-algebra automorphisms via

$$
\left(a_{1}, \ldots, a_{m}, b_{1}, \ldots, b_{n}\right) \cdot Y_{i, \alpha}=a_{i} b_{\alpha} Y_{i, \alpha} \quad \text { for all }(i, \alpha) \in \llbracket 1, m \rrbracket \times \llbracket 1, n \rrbracket .
$$

Moreover, since $q$ is not a root of unity, $R$ endowed with this action of $\mathscr{H}$ is a uniparameter CGL extension (see for instance [Launois et al. 2006]). Before going any further let us describe the antisymmetric matrix associated to the uniparameter CGL extension $\mathrm{O}_{q}\left(M_{m, n}\right)$; it is given by

$$
B=\left(b_{k, l}\right):=\left(\begin{array}{ccccc}
A & I_{m} & I_{m} & \ldots & I_{m} \\
-I_{m} & A & I_{m} & \ldots & I_{m} \\
\vdots & \ddots & \ddots & \ddots & \vdots \\
-I_{m} & \ldots & -I_{m} & A & I_{m} \\
-I_{m} & \ldots & \ldots & -I_{m} & A
\end{array}\right) \in M_{m n}(\mathbb{Q}),
$$

where $I_{m}$ denotes the identity matrix of $M_{m}(\mathbb{Q})$ and

$$
A:=\left(\begin{array}{rrrrr}
0 & 1 & 1 & \ldots & 1 \\
-1 & 0 & 1 & \ldots & 1 \\
\vdots & \ddots & \ddots & \ddots & \vdots \\
-1 & \ldots & -1 & 0 & 1 \\
-1 & \ldots & \ldots & -1 & 0
\end{array}\right) \in M_{m}(\mathbb{Z}) \subseteq M_{m}(\mathbb{Q})
$$

That $R=\mathrm{O}_{q}\left(M_{m, n}\right)$ is a (uniparameter) CGL extension implies in particular that $\mathscr{H}$ - $\operatorname{Spec}(R)$ is finite and that every $\mathscr{H}$-prime is completely prime. Also, as $R=\mathrm{O}_{q}\left(M_{m, n}\right)$ is a CGL extension, one can apply the results of Section 2 to this algebra. In particular, using the theory of deleting derivations, Cauchon has given a combinatorial description of $\mathscr{H}-\operatorname{Spec}(R)$. More precisely, in the case of the algebra $R=\mathrm{O}_{q}\left(M_{m, n}\right)$, he has described the set $W^{\prime}$ that appeared in Section $2 \mathrm{C}$ as follows.

First, it follows from [Cauchon 2003b, Section 2.2] that the quantum affine space $\bar{R}$ that appears in Section $2 \mathrm{C}$ is in this case $\bar{R}=\mathbb{K}_{\Lambda}\left[T_{1,1}, T_{1,2}, \ldots, T_{m, n}\right]$, where $\Lambda$ denotes the $m n \times m n$ matrix whose entries are defined by $\Lambda_{k, l}=q^{b_{k, l}}$ for all $k, l \in \llbracket 1, m n \rrbracket$. Using the canonical embedding (see Section 2C), Cauchon [2003b] produced a bijection between $\mathscr{H}-\operatorname{Spec}\left(\mathscr{O}_{q}\left(M_{m, n}\right)\right)$ and the collection $\mathscr{C}_{m, n}$ of $m \times n$ Cauchon diagrams as defined on page 177 . Roughly speaking, the set $W^{\prime}$ defined on page 184 coincides with $\mathscr{C}_{m, n}$. 
Let us make this precise. If $C$ is an $m \times n$ Cauchon diagram in the sense of the definition on page 177 , we denote by $K_{C}$ the (completely) prime ideal of $\bar{R}$ generated by the indeterminates $T_{i, \alpha}$ such that the box in position $(i, \alpha)$ is a black box of $C$. Then, with $\varphi: \operatorname{Spec}(R) \rightarrow \operatorname{Spec}(\bar{R})$ denoting the canonical embedding, it follows from [Cauchon 2003b, Corollaire 3.2.1] that there exists a unique $\mathscr{H}$ invariant (completely) prime ideal $J_{C}$ of $R$ such that $\varphi\left(J_{C}\right)=K_{C}$; moreover there are no other $\mathscr{H}$-primes in $\mathcal{O}_{q}\left(M_{m, n}\right)$; that is,

$$
\mathscr{H}-\operatorname{Spec}\left(\mathscr{O}_{q}\left(M_{m, n}\right)\right)=\left\{J_{C} \mid C \in \mathscr{C}_{m, n}\right\} .
$$

This last equality justifies the terminology " $m \times n$ Cauchon diagrams" for the combinatorial objects defined on page 177.

In light of this, the containment rule for $m \times n$ Cauchon diagrams given in the Introduction coincides exactly with set-theoretic containment for the more general description of Cauchon diagrams in terms of sets. So, in the case of quantum matrices, Theorem 2.3 can be rephrased as follows.

Theorem 4.1. If $C$ and $C^{\prime}$ are two $m \times n$ Cauchon diagrams with $C \subsetneq C^{\prime}$, then $J_{C} \subsetneq$ $J_{C^{\prime}}$, where $J_{C}$ and $J_{C^{\prime}}$ denote respectively the He-primes of $\mathrm{O}_{q}\left(M_{m, n}\right)$ associated to $C$ and $C^{\prime}$.

4B. Dimension of $\mathscr{H}$-strata. We now give some results about the dimension of the $\mathscr{H}$-stratum of an $\mathcal{H}$-prime of $\mathcal{O}_{q}\left(M_{m, n}\right)$ corresponding to an $m \times n$ Cauchon diagram $C$. We give two related definitions.

A Cauchon diagram $C$ is labeled if each white box in $C$ is labeled with a positive integer such that: the labels are strictly increasing from left to right along rows, and if $i<j$ then the label of each white box in row $i$ is strictly less than the label of each white box in row $j$.

Let $C$ be an $m \times n$ labeled Cauchon diagram with $d$ white boxes and labels $l_{1}<\cdots<l_{d}$. We define the skew-adjacency matrix, $M(C)$, of $C$ to be the $d \times d$ matrix whose $(i, j)$ entry is:

(1) 1 if the box labeled $l_{i}$ is strictly to the left and in the same row as the box labeled $l_{j}$ or is strictly above and in the same column as the box labeled $l_{j}$;

(2) -1 if the box labeled $l_{i}$ is strictly to the right and in the same row as the box labeled $l_{j}$ or is strictly below and in the same column as the box labeled $l_{j}$;

(3) 0 otherwise.

Observe that $M(C)$ is independent of the set of labels which appear in $C$. See, for example, Figure 3.

As a particular case of Theorem 3.1, we get the following result for the uniparameter CGL extension $\mathrm{O}_{q}\left(M_{m, n}\right)$. 


$$
C: \begin{array}{|lll|l}
\hline & 1 & & \\
& 3 & 4 & 5 \\
& & &
\end{array} \quad \mapsto \quad M(C)=\left(\begin{array}{rrrrr}
0 & 0 & 1 & 0 & 0 \\
0 & 0 & 0 & 1 & 0 \\
-1 & 0 & 0 & 1 & 1 \\
0 & -1 & -1 & 0 & 1 \\
0 & 0 & -1 & -1 & 0
\end{array}\right)
$$

Figure 3. A labeled Cauchon diagram $C$ and its corresponding skew-adjacency matrix $M(C)$.

Proposition 4.2. Let $C$ be an $m \times n$ Cauchon diagram. The He-stratum associated to $J_{C}$ is homeomorphic to the prime spectrum of a commutative Laurent polynomial ring over $\mathbb{K}$ in $\operatorname{dim}_{\mathbb{Q}}(\operatorname{ker}(M(C)))$ indeterminates.

We now use this result in order to prove the first part of Theorem 1.1. In order to achieve this aim, we need the following lemma.

Lemma 4.3. Let $C$ be an $m \times n$ labeled Cauchon diagram with $n \leq m$ and with $d$ white boxes with labels $\llbracket 1, d \rrbracket$. Assume that $C$ has no all-black columns. For $1 \leq j \leq n$, let $a_{j}$ denote the smallest label which appears in column $j$ of $C$. Then there is a $d \times d$ lower triangular matrix $S$ such that the matrix obtained by deleting columns $a_{1}, \ldots, a_{n}$ and rows $a_{1}, \ldots, a_{n}$ from $S \cdot M(C)$ is invertible.

Proof. We let $d_{i}$ denote the number of white boxes in the $i$-th row of $C$. Then

$$
d=d_{1}+\cdots+d_{m}
$$

and we can write $M(C)$ in block form as

$$
M(C)=\left(\begin{array}{cccc}
A_{1} & J_{1,2} & \cdots & J_{1, m} \\
-J_{1,2}^{\mathrm{T}} & A_{2} & \cdots & J_{2, m} \\
\vdots & \vdots & \ddots & \vdots \\
-J_{1, m}^{\mathrm{T}} & -J_{2, m}^{\mathrm{T}} & \cdots & A_{m}
\end{array}\right),
$$

where $A_{i}$ is the $d_{i} \times d_{i}$ matrix whose diagonal entries are zero, whose entries above the diagonal are 1 , and whose entries below the diagonal are -1 ; and $J_{i, j}$ is the $d_{i} \times d_{j} 0,1$-matrix whose $(k, l)$ entry is 1 if the $k$-th white element in row $i$ of $C$ (looking from left to right) is directly above the $l$-th white element in row $j$ of $C$ (again, looking left to right) and is 0 otherwise.

We now define a $d \times d$ lower-triangular matrix $S$, whose diagonal entries are all 1 and for $i>j$, whose $(i, j)$ entry is -1 if the white box labeled $i$ is in the $k$-th column of $C$ and $j=a_{k}$; and whose $(i, j)$ entry is 0 otherwise. We now consider the product $S \cdot M(C)$.

Claim. If $i<j$ and the boxes labeled $i$ and $j$ are not in the same row of $C$ and $i \notin\left\{a_{1}, \ldots, a_{n}\right\}$ then the $(i, j)$ entry of $S \cdot M(C)$ is zero. 
Proof of the claim. Suppose $i<j$ and $i \notin\left\{a_{1}, \ldots, a_{n}\right\}$. Let $l$ be the column in which the box labeled $i$ in $C$ sits. Then

$$
\begin{aligned}
(S \cdot M(C))_{i, j} & =\sum_{k=1}^{d} S_{i, k} M(C)_{k, j} \\
& =S_{i, i} M(C)_{i, j}+S_{i, a_{l}} M(C)_{i, j}=M(C)_{i, j}-M(C)_{a_{l}, j} .
\end{aligned}
$$

By assumption, the boxes labeled $i$ and $j$ are not in the same row of $C$ and hence if $M(C)_{i, j}=1$, then $j$ must also be in the $l$-th column of $C$. But then $M(C)_{a_{l}, j}=1$, and so $(S \cdot M(C))_{i, j}=0$. Similarly, if $M(C)_{i, j}=0$, then $j$ must be in a different column than $i$ and so $M(C)_{a_{l}, j}=0$ as well. The claim follows.

Let $D$ denote the matrix obtained from $S \cdot M(C)$ by deleting the rows indexed by $a_{1}, \ldots, a_{n}$ and the columns indexed by $a_{1}, \ldots, a_{n}$. Let $e_{i}$ denote the number of labels $\left\{a_{1}, \ldots, a_{n}\right\}$ which appear in the $i$-th row of $C$. By the claim above, $D$ is a block lower-triangular matrix; that is,

$$
D=\left(\begin{array}{cccc}
D_{1} & 0 & \cdots & 0 \\
* & D_{2} & \cdots & 0 \\
\vdots & \vdots & \ddots & \vdots \\
* & * & \cdots & D_{m}
\end{array}\right)
$$

where $D_{i}$ is a $\left(d_{i}-e_{i}\right) \times\left(d_{i}-e_{i}\right)$ matrix.

Claim. For $1 \leq i \leq m$, we have $D_{i}=-I_{d_{i}-e_{i}}+D_{i}^{\prime}$ for some skew-symmetric matrix $D_{i}^{\prime}$.

Proof of the claim. We first consider the matrix $S \cdot M(C)$. Let $\{a+1, a+2, \ldots$, $\left.a+d_{k}\right\}$ be the set of labels in the $k$-th row of $M(C)$. (Here $a=d_{1}+d_{2}+\cdots+d_{k-1}$.) Consider $(S \cdot M(C))_{a+i, a+j}$. Since $D$ is obtained by deleting the rows and columns of $S \cdot M(C)$ indexed by $a_{1}, \ldots, a_{d}$, we may assume that $a+i \notin\left\{a_{1}, \ldots, a_{n}\right\}$. In this case, the box labeled $a+i$ appears in the $l$-th column of $C$ for some $l$. Then $(S \cdot M(C))_{a+i, a+j}=M(C)_{a+i, a+j}-M(C)_{a_{l}, a+j}$. Observe that $M(C)_{a_{l}, a+j}$ is nonzero if and only if $a+j$ is also in the $l$-th column of $C$; but by assumption, $a+i$ is in the $l$-th column and the boxes labeled $a+i$ and $a+j$ are in the same row, so this is impossible unless $i=j$. Hence

$$
(S \cdot M(C))_{a+i, a+j}=M(C)_{a+i, a+j}-\delta_{i, j},
$$

if $a+i \notin\left\{a_{1}, \ldots, a_{n}\right\}$. To obtain $D$, we simply delete the rows and columns indexed by $\left\{a_{1}, \ldots, a_{n}\right\}$. In particular, if we let $A_{k}$ denote the $d_{k} \times d_{k}$ submatrix of $A$ whose rows and columns are indexed by $a+1, \ldots, a+d_{k}$ (see (4.15)), then $D_{k}$ is obtained by deleting the $e_{i}$ rows and columns indexed by the labels $\left\{a_{1}, \ldots, a_{n}\right\}$ 
which occur in the $i$-th row of $C$ and then subtracting the identity. Since each $A_{k}$ is skew-symmetric, the claim follows.

We have seen that the matrix $D$ is indeed a block lower-triangular matrix; thus to finish the proof, it is enough to show that each $D_{i}$ is invertible. To see this, note that it is of the form $-I+D_{i}^{\prime}$ for some real skew-symmetric matrix $D_{i}^{\prime}$. Since the nonzero eigenvalues of a real skew-symmetric matrix are purely imaginary, we see that $D_{i}$ cannot have any eigenvalue equal to zero and hence is invertible. It follows that the matrix $D$ is invertible.

We are now in position to prove the first part of Theorem 1.1.

Theorem 4.4. Let $C$ be an $m \times n$ Cauchon diagram with $n \leq m$. Then

$$
\operatorname{dim}(\operatorname{ker}(M(C))) \leq n .
$$

As a consequence the dimension of the He-stratum associated to $J_{C}$ is at most $n$.

Proof. Assume first that $C$ has no all-black columns. By Lemma 4.3, there exists an invertible matrix $S$ such that deleting $n$ rows and $n$ columns from $S \cdot M(C)$ gives an invertible matrix. This means that $\operatorname{dim}(\operatorname{ker}(S \cdot M(C))) \leq n$. Moreover $\operatorname{dim}(\operatorname{ker}(M(C)))=\operatorname{dim}(\operatorname{ker}(S \cdot M(C)))$, since $S$ is invertible. The result follows in this case.

Assume now that $C$ has at least one all-black column. Let $\widehat{C}$ be the Cauchon diagram obtained from $C$ by removing every all-black column of $C$. Then $M(C)=$ $M(\widehat{C})$, and so the result follows from the previous case.

We can now prove Theorem 1.1.

Theorem 4.5. Let $m$ and $n$ be natural numbers. Then the dimensions of H-strata in $\mathrm{O}_{q}\left(M_{m, n}\right)$ are all at most $\min (m, n) ;$ moreover, for each $d \in\{0,1, \ldots, \min (m, n)\}$ there exists a d-dimensional He-stratum.

Proof. We assume that $n \leq m$. By Theorem 4.4, the dimensions of the $\mathscr{H}$-strata are all at most $n$ and so it is sufficient to show that each of these values can occur. Let $d \leq n$. We take $P$ to be the $\mathscr{H}$-prime corresponding to the $m \times n$ Cauchon diagram whose $(i, j)$ square is white if and only if $i$ and $j$ are both at most $d$. Then $\mathrm{O}_{q}\left(M_{m, n}\right) / P$ is isomorphic to the ring of $d \times d$ quantum matrices. It follows from [Launois and Lenagan 2007, Theorem 2.5] that the dimension of the stratum associated to $P$ is exactly $d$. This completes the proof.

4C. Proof of Theorem 1.2. In this section we use Theorem 4.1 along with Theorem 4.5 to prove Theorem 1.2.

We first make a remark that will be useful in proving the next proposition.

Remark. Let $A$ be an $n \times n$ real skew-symmetric matrix. Then the dimension of the kernel of $A$ has the same parity as $n$. 


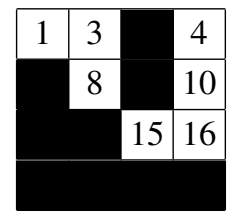

Figure 4. An example of a $4 \times 4$ labeled Cauchon diagram.

Proposition 4.6. Let $C$ be an $m \times n$ Cauchon diagram with $n \leq m$. Suppose that the kernel of $M(C)$ has dimension $e \geq 1$. Then there is a Cauchon diagram $C^{\prime} \supseteq C$ obtained by adding exactly one black box to $C$ such that $M\left(C^{\prime}\right)$ has an $(e-1)$ dimensional kernel.

Proof. Clearly we can assume that $C$ has no all-black columns. Let $d$ be the number of white boxes in $C$. We make $C$ into an $m \times n$ labeled Cauchon diagram with labels $\llbracket 1, d \rrbracket$.

Let $T=\left\{i_{1}, \ldots, i_{k}\right\}$ denote the set of all labels of white boxes of $C$ with the property that if one of these labels is coloured black and the remaining boxes of $C$ are left unchanged then the result is still a Cauchon diagram.

For example, in Figure 4 the labels of the white boxes which can be coloured black to obtain a Cauchon diagram are 1, 3, 4, 8 and 15 .

Given $i \in T$, we let $C_{i}$ denote the Cauchon diagram obtained by colouring the white box with label $i$ black.

If $\operatorname{dim}\left(\operatorname{ker}\left(M\left(C_{i}\right)\right)\right) \geq e$ for every $i \in T$, then by parity considerations (see remark immediately above) we must have $\operatorname{dim}\left(\operatorname{ker}\left(M\left(C_{i}\right)\right)\right) \geq e+1$, for $i \in T$. Let

$$
\left\{v_{1}^{(i)}, \ldots, v_{e+1}^{(i)}\right\}
$$

be a linearly independent set of vectors in the kernel of $M\left(C_{i}\right)$. We construct $e+1$ vectors as follows. For $1 \leq j \leq e+1$, let $w_{j}^{(i)}$ be the $d \times 1$ column vector whose $l$-th coordinate is the $l$-th coordinate of $v_{j}^{(i)}$ if $l<i$, is 0 if $l=i$, and is the $(l-1)$-th coordinate of $v_{j}^{(i)}$ if $l>i$. By construction, every row of $M(C)$ is orthogonal to the linearly independent set $\left\{w_{1}^{(i)}, \ldots, w_{e+1}^{(i)}\right\}$ except for possibly the $i$-th row. Let $r$ denote the $i$-th row of $M(C)$. Then $r$ is an $1 \times d$ row vector. We then have a map

$$
\operatorname{Span}\left(\left\{w_{1}^{(i)}, \ldots, w_{e+1}^{(i)}\right\}\right) \rightarrow \mathbb{Q}
$$

in which a vector $w$ in the span is sent to $r \cdot w \in \mathbb{Q}$. This map is surjective since otherwise the dimension of the kernel of $M(C)$ would be at least $(e+1)$-dimensional. Thus the kernel of this map is an $e$-dimensional subspace of

$$
\operatorname{Span}\left(\left\{w_{1}^{(i)}, \ldots, w_{e+1}^{(i)}\right\}\right)
$$

which lies in the kernel of $M(C)$. Since the kernel of $M(C)$ is exactly $e$-dimensional, and every vector in $\operatorname{Span}\left(\left\{w_{1}^{(i)}, \ldots, w_{e+1}^{(i)}\right\}\right)$ has a zero in the $i$-th coordinate, 
we see that every vector in $\operatorname{ker}(M(C))$ has a zero in the $i$-th coordinate; moreover, this is the case for every $i \in T$. For $1 \leq j \leq n$, recall that $a_{j}$ denotes the smallest label which appears in column $j$ of $C$. Then $\left\{a_{1}, \ldots, a_{n}\right\} \subseteq T$, as they are the labels of the uppermost white boxes in each column. By Lemma 4.3 there is a lower-triangular matrix $S$ such that $S \cdot M(C)$ has the property that if columns $a_{1}, \ldots, a_{n}$ and rows $a_{1}, \ldots, a_{n}$ are deleted then the resulting matrix is invertible. Let $\left\{v_{1}, \ldots, v_{e}\right\}$ be a basis for $\operatorname{ker}(M(C))$. For each $i \leq e$, let $u_{i}$ denote the $(d-n) \times 1$ column vector obtained by taking the $d \times 1$ vector $v_{i}$ and simply removing coordinates $a_{1}$ through $a_{n}$. Since the $a_{j}$-th coordinate of $v_{i}$ is 0 for each $i$ and $j$, we see that $u_{1}, \ldots, u_{e}$ are linearly independent and are in the kernel of the matrix obtained by removing columns $a_{1}, \ldots, a_{n}$ from $S \cdot M(C)$. Thus these vectors are in the kernel of the matrix obtained by deleting columns $a_{1}, \ldots, a_{n}$ and rows $a_{1}, \ldots, a_{n}$ of $S \cdot M(C)$. But this contradicts the fact that the matrix obtained by deleting columns $a_{1}, \ldots, a_{n}$ and rows $a_{1}, \ldots, a_{n}$ of $S \cdot M(C)$ is invertible. It follows that there is some $i \in T$ such that the Cauchon diagram $C_{i}$ has $\operatorname{dim}\left(\operatorname{ker}\left(M\left(C_{i}\right)\right) \leq e-1\right.$. We claim that

$$
\operatorname{dim}\left(\operatorname{ker}\left(M\left(C_{i}\right)\right)=e-1 .\right.
$$

To see this, observe that it is no loss of generality to assume that in the basis $\left\{v_{1}, \ldots, v_{e}\right\}$ of $\operatorname{ker}(M(C))$ the vectors $v_{1}, \ldots, v_{e-1}$ have a zero $i$-th coordinate. Then the vectors $v_{1}^{\prime}, \ldots, v_{e-1}^{\prime}$ obtained by deleting the $i$-th coordinate from $v_{1}, \ldots, v_{e-1}$ are in the kernel of $M\left(C_{i}\right)$ and are linearly independent. The result follows.

Recall that an $\mathscr{H}$-prime ideal in $R=\mathrm{O}_{q}\left(M_{m, n}\right)$ is primitive if and only if its associated $\mathscr{H}$-stratum is 0-dimensional [Brown and Goodearl 2002, Theorem II.8.4].

Theorem 4.7. Let $J_{C}$ be the H-prime of $R=O_{q}\left(M_{m, n}\right)$ associated to the Cauchon diagram $C$. Suppose that the dimension of the He-stratum associated to $J_{C}$ is equal to $e$. Then there is a chain $C=C_{0} \subsetneq C_{1} \subsetneq \cdots \subsetneq C_{e}$ of $m \times n$ Cauchon diagrams such that $J_{C}=J_{C_{0}} \subsetneq J_{C_{1}} \subsetneq \cdots \subsetneq J_{C_{e}}$ and, for all $i$, the dimension of the $\mathscr{H}$ stratum associated to $J_{C_{i}}$ is $e-i$. In particular, $J_{C_{e}}$ is a primitive He-prime ideal in $\mathrm{O}_{q}\left(M_{m, n}\right)$.

Proof. We prove this by induction on $e$. If $e=0$, then $J_{C}$ is primitive and there is nothing to prove.

Suppose now that $e>0$. By Proposition 4.6, there exists a Cauchon diagram $C_{1}$ obtained by turning a single white box of $C$ black such that $\operatorname{ker}\left(M\left(C_{1}\right)\right)$ has dimension $e-1$. As $C=C_{0} \subsetneq C_{1}$, it follows from Theorem 4.1 that $J_{C}=J_{C_{0}} \subsetneq$ $J_{C_{1}}$. Moreover it follows from Proposition 4.2 that the dimension of the $\mathscr{H}$-stratum associated to $J_{C_{1}}$ is equal to $\operatorname{dim}\left(\operatorname{ker}\left(M\left(C_{1}\right)\right)\right)=e-1$. The result is obtained by applying the induction hypothesis to $J_{C_{1}}$. 


\section{A conjecture}

Our results show that the possible dimensions of the $\mathscr{H}$-strata of $\mathcal{O}_{q}\left(M_{m, n}\right)$ are $\{0,1,2, \ldots, \min (m, n)\}$; moreover, every one of these values occurs as the dimension of some $\mathscr{H}$-stratum. What is still unresolved, however, is how exactly the dimensions of $\mathscr{H}$-strata of $\mathcal{O}_{q}\left(M_{m, n}\right)$ are distributed. Earlier, we investigated the enumeration of 0-dimensional $\mathscr{H}$-strata in $\mathrm{O}_{q}\left(M_{m, n}\right)$ [Bell et al. 2009; Bell et al. 2010]; this is the same as enumerating the primitive $\mathscr{H}$-primes of $\mathrm{O}_{q}\left(M_{m, n}\right)$. We make a general conjecture about the proportion of $i$-dimensional $\mathscr{H}$-strata in $\mathrm{O}_{q}\left(M_{m, n}\right)$ with $i \leq m$ and $n \geq m$.

Conjecture 5.1. Let $m$ be a natural number. Then for $0 \leq i \leq m$ we have

$$
\lim _{n \rightarrow \infty} \frac{\# i \text {-dimensional } \mathscr{H} \text {-strata in } O_{q}\left(M_{m, n}\right)}{\# \mathscr{H} \text {-strata in } O_{q}\left(M_{m, n}\right)}=2^{1-\delta_{i, 0}}\left(\begin{array}{c}
2 m \\
m+i
\end{array}\right) 4^{-m} .
$$

We have shown this for $(i, m) \in\{(0,1),(1,1),(0,2),(1,2),(2,2),(0,3),(2,3)\}$; moreover, extensive computer computations suggest this is true in general.

\section{Acknowledgment}

We thank the anonymous referee for comments that have greatly improved this text.

\section{References}

[Bell et al. 2009] J. Bell, S. Launois, and N. Nguyen, "Dimension and enumeration of primitive ideals in quantum algebras", J. Algebraic Combinatorics 29:3 (2009), 269-294. MR 2496308 Zbl 05551278

[Bell et al. 2010] J. Bell, S. Launois, and J. Lutley, "An automaton-theoretic approach to the representation theory of quantum algebras", Adv. Math. 223:2 (2010), 476-510. Zbl 05653475

[Brown and Goodearl 1996] K. A. Brown and K. R. Goodearl, "Prime spectra of quantum semisimple groups”, Trans. Amer. Math. Soc. 348:6 (1996), 2465-2502. MR 96i:17007 Zbl 0857.16026

[Brown and Goodearl 2002] K. A. Brown and K. R. Goodearl, Lectures on algebraic quantum groups, Birkhäuser, Basel, 2002. MR 2003f:16067 Zbl 1027.17010

[Cauchon 2003a] G. Cauchon, "Effacement des dérivations et spectres premiers des algèbres quantiques”, J. Algebra 260:2 (2003), 476-518. MR 2004g:16044 Zbl 1017.16017

[Cauchon 2003b] G. Cauchon, "Spectre premier de $O_{q}\left(M_{n}(k)\right)$ : image canonique et séparation normale”, J. Algebra 260:2 (2003), 519-569. MR 2004g:16045 Zbl 1024.16001

[Cauchon and Mériaux 2009] G. Cauchon and A. Mériaux, "Admissible diagrams in $U_{q}^{w} \mathfrak{g}$ ) and combinatoric properties of Weyl groups", preprint, 2009. arXiv 0902.0754

[De Concini and Procesi 1993] C. De Concini and C. Procesi, "Quantum groups", pp. 31-140 in Dmodules, representation theory, and quantum groups (Venice, 1992), edited by G. Zampieri and A. D’Agnolo, Lecture Notes in Math. 1565, Springer, Berlin, 1993. MR 95j:17012 Zbl 0795.17005 
[Goodearl and Letzter 1994] K. R. Goodearl and E. S. Letzter, "Prime factor algebras of the coordinate ring of quantum matrices", Proc. Amer. Math. Soc. 121:4 (1994), 1017-1025. MR 94j:16066 Zbl 0812.16039

[Goodearl and Letzter 1998] K. R. Goodearl and E. S. Letzter, "Prime and primitive spectra of multiparameter quantum affine spaces", pp. 39-58 in Trends in ring theory (Miskolc, 1996), edited by V. Dlab and L. Márki, CMS Conf. Proc. 22, Amer. Math. Soc., Providence, RI, 1998. MR 99h:16045 Zbl 0904.16001

[Goodearl and Letzter 2000] K. R. Goodearl and E. S. Letzter, "The Dixmier-Moeglin equivalence in quantum coordinate rings and quantized Weyl algebras", Trans. Amer. Math. Soc. 352:3 (2000), 1381-1403. MR 2000j:16040 Zbl 0978.16040

[Jantzen 1996] J. C. Jantzen, Lectures on quantum groups, Graduate Studies in Mathematics 6, American Mathematical Society, Providence, RI, 1996. MR 96m:17029 Zbl 0842.17012

[Joseph 1995] A. Joseph, Quantum groups and their primitive ideals, Ergebnisse der Mathematik 29, Springer, Berlin, 1995. MR 96d:17015 Zbl 0808.17004

[Launois 2007] S. Launois, "Combinatorics of $\mathscr{H}$-primes in quantum matrices", J. Algebra 309:1 (2007), 139-167. MR 2007k:05221 Zbl 1172.05051

[Launois and Lenagan 2007] S. Launois and T. H. Lenagan, "Primitive ideals and automorphisms of quantum matrices", Algebras and Representation Theory 10:4 (2007), 339-365. MR 2008e:16043 Zbl 1124.16037

[Launois et al. 2006] S. Launois, T. H. Lenagan, and L. Rigal, "Quantum unique factorisation domains”, J. London Math. Soc. (2) 74:2 (2006), 321-340. MR 2007h:16047 Zbl 1116.16040

[Launois et al. 2008] S. Launois, T. H. Lenagan, and L. Rigal, "Prime ideals in the quantum Grassmannian”, Selecta Math. (N.S.) 13:4 (2008), 697-725. MR 2009e:20110 Zbl 1146.16023

[Levendorskiı̌ and Soibelman 1991] S. Levendorskiı̌ and Y. Soibelman, "Algebras of functions on compact quantum groups, Schubert cells and quantum tori”, Comm. Math. Phys. 139:1 (1991), 141-170. MR 92h:58020 Zbl 0729.17011

[Lusztig 1993] G. Lusztig, Introduction to quantum groups, Progress in Mathematics 110, Birkhäuser, Boston, MA, 1993. MR 94m:17016 Zbl 0788.17010

[Mériaux 2010] A. Mériaux, "Cauchon diagrams for quantized enveloping algebras", J. Algebra 323:4 (2010), 1060-1097.

[Yakimov 2009] M. Yakimov, "Invariant prime ideals in quantizations of nilpotent Lie algebras", preprint, 2009. arXiv 0905.0852

Communicated by Susan Montgomery

Received 2009-03-09 Revised 2009-10-14 Accepted 2009-11-26

jpb@math.sfu.ca

Jason Bell, Department of Mathematics, Simon Fraser University, Burnaby, BC V5A 1S6, Canada

s.launois@kent.ac.uk School of Mathematics, Statistics and Actuarial science, University of Kent, Canterbury, Kent CT2 7NF, United Kingdom 


\title{
Gentle algebras arising from surface triangulations
}

\author{
Ibrahim Assem, Thomas Brüstle, \\ Gabrielle Charbonneau-Jodoin and Pierre-Guy Plamondon
}

\begin{abstract}
We associate an algebra $A(\Gamma)$ to a triangulation $\Gamma$ of a surface $S$ with a set of boundary marking points. This algebra $A(\Gamma)$ is gentle and Gorenstein of dimension one. We also prove that $A(\Gamma)$ is cluster-tilted if and only if it is cluster-tilted of type $\mathbb{A}$ or $\tilde{A}$, or if and only if the surface $S$ is a disc or an annulus. Moreover all cluster-tilted algebras of type $\mathbb{A}$ or $\tilde{A}$ are obtained in this way.
\end{abstract}

\section{Introduction}

Among the main recent results in the fast-growing theory of cluster algebras is the paper of Fomin, Shapiro and Thurston [Fomin et al. 2008], relating triangulations of oriented surfaces to cluster algebras. This approach, which existed since the beginning of the theory [Caldero et al. 2006], was followed in [Labardini-Fragoso 2009; Schiffler 2008], among others. In the same spirit, we consider in the present paper an unpunctured oriented surface $S$ and a finite set of points $M$, lying on the boundary of $S$ and intersecting every boundary component of $S$. We then associate to a triangulation $\Gamma$ of the marked surface $(S, M)$ a quiver $Q(\Gamma)$, and a potential on $Q(\Gamma)$ (in the sense of [Derksen et al. 2008]), thus defining an algebra $A(\Gamma)$, namely the (noncompleted) Jacobian algebra defined by $Q(\Gamma)$ and the associated potential.

Such an algebra $A(\Gamma)$ has some very nice properties: it is always Gorenstein of dimension one, and also it is a gentle algebra in the sense of [Assem and Skowroński 1987]. In the unpunctured case studied here, our definition coincides with Labardini's definition of a quiver with potential associated to a (possibly punctured) surface [Labardini-Fragoso 2009]. But in the punctured case, one does not

MSC2000: primary 16S99; secondary 16G20, 57N05, 57M50.

Keywords: bordered surface with marked points, triangulated surface, quiver with potential, gentle algebra.

Assem is partially supported by NSERC of Canada and the Université de Sherbrooke. Charbonneau was working under a summer research fellowship of NSERC. Brüstle is partially supported by NSERC, by Bishop's University and the Université de Sherbrooke. Plamondon was supported by an NSERC graduate fellowship. 
get gentle algebras, or even string algebras. For instance, a once-punctured disc gives rise to cluster-tilted algebras of type $\mathbb{D}$ [Schiffler 2008].

Gentle algebras form a particularly nice subclass of the class of string algebras of [Butler and Ringel 1987] and are much investigated in the representation theory of algebras. For instance, this subclass contains the tilted algebras of type $\mathbb{A}$ and $\widetilde{A}$ (see [Assem 1982] and [Assem and Skowroński 1987], respectively) and it is closed under tilting and even under derived equivalence (see [Schröer 1999] and [Schröer and Zimmermann 2003], respectively).

Our objective in this paper is twofold. Firstly, we ask which gentle algebras arise in this way, that is, are induced from triangulations of an unpunctured surface with boundary marked points. We show in Proposition 2.8 that this is the case for every gentle algebra such that every relation lies on what we call a 3-cycle with radical-square zero (see definition before Theorem 2.7 or [Buan and Vatne 2008] for the definition). Secondly, we ask which gentle algebras are cluster-tilted. The class of cluster-tilted algebras, introduced in [Buan et al. 2007], has been much investigated and is by now well-understood (see, for instance, [Assem et al. 2008a; Barot et al. 2008; Buan et al. 2006; Buan and Vatne 2008; Caldero et al. 2006; Keller 2009; Keller and Reiten 2007; Schiffler 2008]). In particular, it was shown in [Assem et al. 2008a] that every cluster-tilted algebra is the relation-extension of a tilted algebra, that is, it is the trivial extension of a tilted algebra $C$ by the $C$-C-bimodule $\operatorname{Ext}_{C}^{2}(D C, C)$. We may now state the main result of this paper.

Theorem 1.1. Let $A(\Gamma)$ be the algebra associated to the triangulation $\Gamma$ of an unpunctured marked surface $(S, M)$. Then the following statements are equivalent:

(1) $A(\Gamma)$ is cluster-tilted.

(2) $A(\Gamma)$ is cluster-tilted of type $\mathbb{A}$ and $\widetilde{\mathbb{A}}$.

(3) $A(\Gamma)$ is the relation-extension of a tilted algebra of type $\mathbb{A}$ and $\widetilde{\mathbb{A}}$.

(4) The surface $S$ is a disc or an annulus.

Moreover, all cluster-tilted algebras of type $\mathbb{A}($ or $\widetilde{\mathbb{A}})$ are of the form $A(\Gamma)$ for some triangulation of a disc $S$ (or an annulus $S$, respectively).

Actually, we prove in Theorem 3.3 that a cluster-tilted algebra is gentle if and only if it is of type $\mathbb{A}$ and $\widetilde{A}$, or if and only if it is the relation-extension of a gentle tilted algebra, and the latter coincide with the tilted algebras of type $\mathbb{A}$ or $\widetilde{\mathbb{A}}$, respectively.

The case where $S$ is a disc has already been studied in [Caldero et al. 2006], and it is known that the bound quivers of all cluster-tilted algebras of type $\mathbb{A}$ arise from triangulations of the (unpunctured) disc. These algebras have also been described explicitly in [Buan and Vatne 2008]. Also, the potential we use for defining the cluster-tilted algebras of type $\widetilde{A}$ is a particular case of the potential recently defined 
by Keller [2009]. However, we do not use this fact, but rather present another proof (predating Keller's result), which uses [Assem et al. 2008a] and properties of the second extension group.

The paper is organised as follows: in Section 2, we define our algebras $A(\Gamma)$ and prove their main properties in Theorem 2.7. Section 3 is devoted to the classification of the gentle cluster-titled algebras and Section 4 to the proof of our main theorem and some of its consequences. We conclude with an example of an algebra $A(\Gamma)$ that is not of polynomial growth in the sense of [Skowroński 1990].

\section{Algebras arising from surface triangulations}

Throughout this paper, the algebras we consider are basic connected algebras over a fixed algebraically closed field $k$. Unless otherwise stated, all algebras are finitedimensional. Consequently, they are given in the form $A=k Q / I$ where $Q$ is a quiver and $I$ is an admissible ideal of the path algebra $k Q$ [Assem et al. 2006]. The pair $(Q, I)$ is called a bound quiver, and the algebra $A=k Q / I$ is referred to as a bound quiver algebra.

Given a bound quiver algebra $A=k Q / I$, for every vertex $x$ of $Q$ we denote by $e_{x}$ the idempotent of $A$ associated to $x$. Also, $P_{x}, I_{x}$ and $S_{x}$ will be the corresponding indecomposable projective module, indecomposable injective module and simple module, respectively.

We study in this section the algebra associated with a surface triangulation. For background material on oriented surfaces we refer to [Massey 1991].

The medial quiver $\boldsymbol{Q}(\boldsymbol{\Gamma})$. We first recall from [Fomin et al. 2008] the construction of a quiver for every triangulation of a marked surface. Let $S$ be an oriented surface with boundary $\partial S$, and let $M$ be a nonempty finite set of points on $\partial S$ intersecting each connected component of the boundary $\partial S$. In this paper, we only consider the case where there are no punctures, that is, we request that the set of marked points $M$ be contained in the boundary $\partial S$. The pair $(S, M)$ is referred to as an unpunctured bordered surface with marked points.

An $\operatorname{arc}$ in $(S, M)$ is a curve $\gamma$ in $S$ such that:

- The endpoints of $\gamma$ are marked points in $M$.

- $\gamma$ does not intersect itself, except that its endpoints may coincide.

- $\gamma$ intersects the boundary of $\partial S$ only in its endpoints.

- $\gamma$ does not cut out a monogon (that is, $\gamma$ is not contractible into a point of $M$ ).

We call an arc $\gamma$ a boundary arc if it cuts out a digon (that is, $\gamma$ is homotopic to a curve $\delta$ on the boundary $\partial S$ that intersects $M$ only in its endpoints). Otherwise, $\gamma$ is said to be an internal arc. Each arc $\gamma$ is considered up to homotopy in the 
class of such curves. A triangulation of $(S, M)$ is a maximal collection $\Gamma$ of arcs that do not intersect in the interior of $S$ (more precisely, there are curves in their respective homotopy classes that do not intersect in the interior of $S$ ).

Proposition 2.1 [Fomin et al. 2008, (2.10)]. In each triangulation of $(S, M)$, the number of internal arcs is

$$
n=6 g+3 b+c-6,
$$

where $g$ is the genus of $S, b$ is the number of boundary components, and $c=|M|$ is the number of marked points.

This proposition also indicates that in some cases a triangulation does not exist (for instance a disc with one marked point would give $n=-2$ ). We consider from now on only marked surfaces $(S, M)$ that admit a triangulation. Given a triangulation $\Gamma$, we also refer to $M$ as the set of vertices of $\Gamma$. The triangles are the components of $S \backslash \Gamma$ with the arcs of $\Gamma$ as edges.

We denote by $Q(\Gamma)$ the medial quiver of internal arcs of $\Gamma$. That is, $Q(\Gamma)$ is the quiver whose set of points is the set of internal $\operatorname{arcs}$ of $\Gamma$, and the arrows are defined as follows: whenever there is a triangle $T$ in $\Gamma$ containing two internal arcs $a$ and $b$, then $Q(\Gamma)$ contains an arrow $a \rightarrow b$ if $a$ is a predecessor of $b$ with respect to clockwise orientation at the joint vertex of $a$ and $b$ in $T$ (we can talk about clockwise orientation around each marked point because $S$ is an oriented surface).

Example. We illustrate the construction of $Q(\Gamma)$ when $\Gamma$ is a triangulation of an octagon:
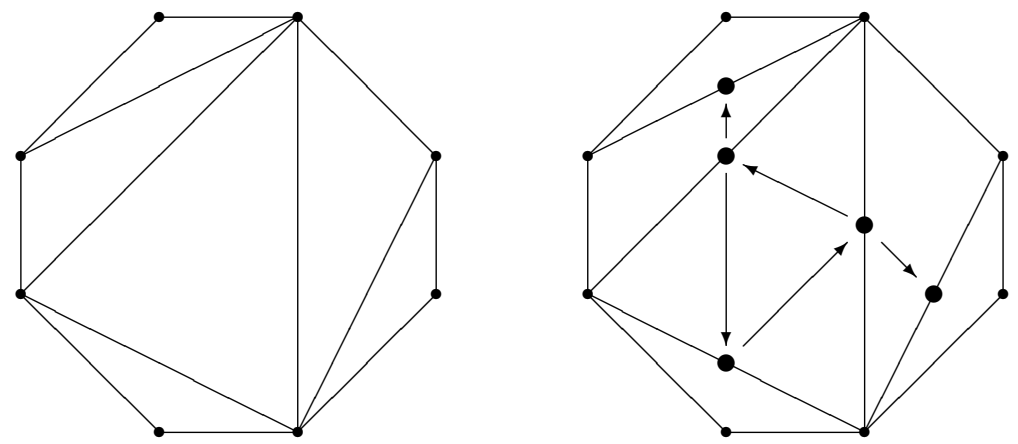

Lemma 2.2. The quiver $Q(\Gamma)$ contains no oriented cycles of length $\leq 2$.

Proof. We first show that $Q(\Gamma)$ contains no loops. A loop $\alpha$ at the point $a$ of $Q(\Gamma)$ would arise from a triangle $T$ in $\Gamma$ in the following way:

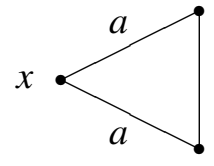

gives rise to

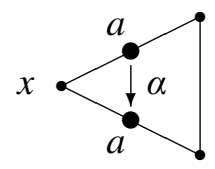


But in this case the triangle $T$ is homeomorphic to

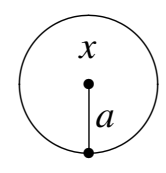

which means that $x$ is an internal vertex, contradicting our assumption that $M$ is contained in the boundary of $S$.

We now show that $Q(\Gamma)$ contains no oriented cycles of length two. Indeed, such a cycle corresponds to the following situation in $\Gamma$ :

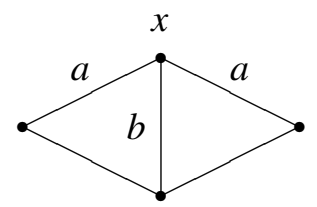

Then a neighbourhood of $x$ is homeomorphic to

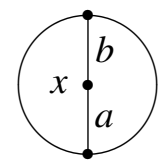

which again contradicts the assumption that $\Gamma$ contains no internal vertices.

Remark. Fomin et al. [2008] associate a skew-symmetric matrix $B(\Gamma)$ to a triangulation $\Gamma$ of $(S, M)$. This construction is equivalent to the construction of the quiver $Q(\Gamma)$ we consider here. Since $Q(\Gamma)$ contains no oriented cycles of length $\leq 2$, it is uniquely determined by a skew-symmetric matrix $B$ (where the number of arrows between two vertices is given by the entries of $B$, and the direction of the arrows is determined by the sign of the matrix entries). It is easy to see that $B$ coincides with $B(\Gamma)$. Thus all the results from [Fomin et al. 2008] apply; in particular, mutations of the quiver $Q(\Gamma)$ correspond to flips of the triangulation $\Gamma$.

Let $b$ be an internal arc of $\Gamma$. Thus $b$ is one diagonal of the quadrilateral formed by the two triangles of $\Gamma$ that contain $b$. The flip of $b$ replaces the edge $b$ by the other diagonal $b^{*}$ of the same quadrilateral. Keeping all other edges unchanged, one obtains a new triangulation $\mu_{b}(\Gamma)$.
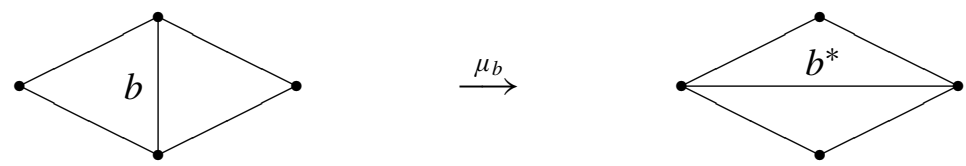

An essential ingredient in the definition of cluster algebras by Fomin and Zelevinsky [2002] is the mutation of skew-symmetric matrices. Reformulated in the language of quivers, one obtains a mutation of quivers $Q \mapsto \mu_{b}(Q)$. The following proposition shows that flips of the triangulation commute with quiver mutations. 
Proposition 2.3 [Fomin et al. 2008, Proposition 4.8]. Suppose that the triangulation $\mu_{b}(\Gamma)$ is obtained from $\Gamma$ by a flip replacing the diagonal labelled $b$. Then

$$
Q\left(\mu_{b}(\Gamma)\right)=\mu_{b}(Q(\Gamma))
$$

The algebra $\boldsymbol{A}(\boldsymbol{\Gamma})$. We define in this section an algebra $A(\Gamma)$ for each triangulation $\Gamma$ of the unpunctured marked surface $(S, M)$. Our construction generalizes the one given in [Caldero et al. 2006] for polygons. An even more general case is considered in [Labardini-Fragoso 2009], where such an algebra $A(\Gamma)$ is defined for a general marked surface (allowing punctures). If there are no punctures, the definitions coincide (although Labardini works in the equivalent framework of opposite medial quivers).

A triangle $T$ in $\Gamma$ is called an internal triangle if all edges of $T$ are internal arcs. Every internal triangle $T$ in $\Gamma$ gives rise to an oriented cycle $\alpha_{T} \beta_{T} \gamma_{T}$ in $Q(\Gamma)$, unique up to cyclic permutation of the factors $\alpha_{T}, \beta_{T}, \gamma_{T}$. We define

$$
W=\sum_{T} \alpha_{T} \beta_{T} \gamma_{T}
$$

where the sum runs over all internal triangles $T$ of $\Gamma$. Then $W$ is a potential on $Q(\Gamma)$ and we define $A(\Gamma)$ to be the (noncompleted) Jacobian algebra of $(Q, W)$ [Derksen et al. 2008; Keller 2007]. Thus $A(\Gamma)$ can be described as a quotient $A(\Gamma)=k Q(\Gamma) / I(\Gamma)$ of the path algebra $k Q(\Gamma)$ by the ideal $I(\Gamma)$ generated by all paths $\alpha_{T} \beta_{T}, \beta_{T} \gamma_{T}$ and $\gamma_{T} \alpha_{T}$ whenever $T$ is an internal triangle of $\Gamma$. Labardini [2009] showed that flips in the triangulation correspond to mutations of the quiver with potential $(Q(\Gamma), W)$ as defined in [Derksen et al. 2008].

The following result is shown in [Labardini-Fragoso 2009, Theorem 36] for the more general case of punctured marked surfaces.

Lemma 2.4. Let $\Gamma$ be a triangulation of an unpunctured marked surface $(S, M)$. Then the algebra $A(\Gamma)$ is finite-dimensional.

We show in Lemma 2.5 that the algebras $A(\Gamma)$ belong to a class of algebras called gentle algebras. Recall from [Assem and Skowroński 1987] that a finitedimensional algebra is gentle if it admits a presentation $A=k Q / I$ satisfying the following conditions:

(G1) At each point of $Q$ start at most two arrows and stop at most two arrows.

(G2) The ideal $I$ is generated by paths of length 2 .

(G3) For each arrow $\beta$ there is at most one arrow $\alpha$ and at most one arrow $\gamma$ such that $\alpha \beta \in I$ and $\beta \gamma \in I$.

(G4) For each arrow $\beta$ there is at most one arrow $\alpha$ and at most one arrow $\gamma$ such that $\alpha \beta \notin I$ and $\beta \gamma \notin I$. 
If the pair $(Q, I)$ satisfies conditions (G1) through (G4), we call it a gentle bound quiver, or a gentle presentation of $A=k Q / I$. Note that in contrast to [Assem and Skowroński 1987], we do not assume that $A=k Q / I$ is triangular. An algebra $A=k Q / I$ where $I$ is generated by paths and $(Q, I)$ satisfies the two conditions (G1) and (G4) is called a string algebra [Butler and Ringel 1987], and thus every gentle algebra is a string algebra. The gentle algebras can be characterized by the fact that their repetitive categories are special biserial [Assem and Skowroński 1987; Pogorzały and Skowroński 1991].

We recall here the classification of indecomposable modules over a string algebra $A=k Q / I$ which is given in [Butler and Ringel 1987] in terms of reduced walks in the quiver $Q$. A string in $A$ is by definition a reduced walk $w$ in $Q$ avoiding the zero-relations, and thus $w$ is a sequence

$$
w=x_{1} \stackrel{\alpha_{1}}{\longleftrightarrow} x_{2} \stackrel{\alpha_{1}}{\longleftrightarrow} \cdots \stackrel{\alpha_{n-1}}{\longleftrightarrow} x_{n},
$$

where the $x_{i}$ are vertices of $Q$ and each $\alpha_{i}$ is an arrow between the vertices $x_{i}$ and $x_{i+1}$ in either direction such that $w$ does not contain a sequence of the form

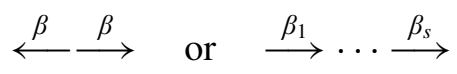

with $\beta_{1} \cdots \beta_{s} \in I$, or their duals. A string is cyclic if the first and the last vertex coincide. A band is defined to be a cyclic string $b$ such that each power $b^{n}$ is a string, but $b$ itself is not a proper power of some string $c$.

The string module $M(w)$ is obtained from the string $w$ by replacing each $x_{i}$ in $w$ by a copy of the field $k$. The action of an arrow $\alpha$ on $M(w)$ is induced by the relevant identity morphisms if $\alpha$ lies on $w$, and is zero otherwise. The dimension vector $\operatorname{dim} M(w)$ of $M(w)$ is obtained by counting how often the string $w$ passes through each vertex $x$ of the quiver $Q$ :

$$
\underline{\operatorname{dim}} M(w)=\left(\sum_{1 \leq i \leq n} \delta_{x, x_{i}}\right)_{x \in Q_{0}},
$$

where $\delta_{x, x_{i}}=1$ for $x=x_{i}$ and $\delta_{x, x_{i}}=0$ otherwise. Similarly, each band $b$ in $A$ gives rise to a family of band modules $M(b, \lambda, n)$ where $\lambda \in k$ and $n \in \mathbb{N}$ (we refer to [Butler and Ringel 1987] for the precise definition). All string and band modules are indecomposable, and in fact every indecomposable $A$-module is either a string module $M(w)$ or a band module $M(b, \lambda, n)$ [Butler and Ringel 1987].

We now return to the study of algebras stemming from surface triangulations:

Lemma 2.5. Let $\Gamma$ be a triangulation of an unpunctured marked surface $(S, M)$. Then $A(\Gamma)$ is a gentle algebra.

Proof. By Lemma 2.4, the algebra $A(\Gamma)$ is finite-dimensional, so we only need to verify conditions $(\mathrm{G} 1)$ to $(\mathrm{G} 4)$ for the bound quiver $(Q(\Gamma), I(\Gamma))$ of $A(\Gamma)$. 
(G2): By definition, the ideal $I(\Gamma)$ is generated by paths of length two.

(G1): Let $a$ be a point of $Q(\Gamma)$ corresponding to an internal $\operatorname{arc} a$ of $\Gamma$. Since $\Gamma$ is a triangulation of a surface, the arc $a$ is contained in at most two triangles:

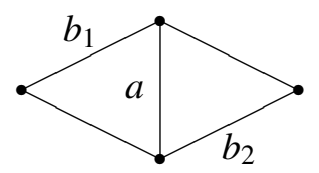

Hence there are at most two arrows $\alpha_{1}: b_{1} \rightarrow a$ and $\alpha_{2}: b_{2} \rightarrow a$ of $Q(\Gamma)$ ending in $a$. The same holds for arrows starting in a point $a$.

(G3), (G4): Suppose now that $Q(\Gamma)$ contains $\alpha_{1}, \alpha_{2}, \beta$ as follows:

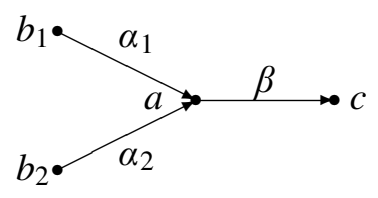

We have to show that precisely one of $\alpha_{1} \beta, \alpha_{2} \beta$ belongs to $I(\Gamma)$. In $\Gamma$, the internal arcs $a, b_{1}, b_{2}$ belong to two triangles as considered in the proof of (G1). The arrow $\beta$ encodes that the $\operatorname{arc} c$ is a successor of $a$ in one of these triangles, say the one formed by $a, b_{1}, c$. This gives rise to the relation $\alpha_{1} \beta$, and $\alpha_{2} \beta$ does not belong to $I(\Gamma)$ since $\alpha_{2}$ and $\beta$ arise from different triangles.

From the construction of $A(\Gamma)$ it is clear that for each $\alpha \beta \in I(\Gamma)$ there is an arrow $\gamma$ in $Q(\Gamma)$ such that $\beta \gamma \in I(\Gamma)$ and $\gamma \alpha \in I(\Gamma)$. In the following lemma we study a homological property of all gentle algebras satisfying this condition: an algebra $A$ is Gorenstein of dimension one if the injective dimension of the (finitely generated) projective $A$-modules is at most one, and the projective dimension of the (finitely generated) injective $A$-modules is at most one. Note that all clustertilted algebras are Gorenstein of dimension one, and that an algebra of Gorenstein dimension one is either hereditary or has infinite global dimension; see [Keller and Reiten 2007].

Lemma 2.6. Let $A=k Q / I$ be a gentle algebra such that for each $\alpha \beta \in I$ there is an arrow $\gamma$ in $Q$ such that $\beta \gamma \in I$ and $\gamma \alpha \in I$. Then $A$ is Gorenstein of dimension one.

Proof. We only compute the projective dimension of the injective modules here; the proof of the other part in the definition of Gorenstein of dimension one is dual. It is sufficient to show that for every vertex $x$ of $Q$ the corresponding indecomposable injective $A$-module $I_{x}$ has projective dimension at most one. To do so, we construct explicitly a projective resolution of $I_{x}$. We write the string module $I_{x}$ as $I_{x}=$ $M\left(u_{1} \alpha_{1} \alpha_{2}^{-1} u_{2}^{-1}\right)$, where $u_{1}$ and $u_{2}$ are oriented paths. Both paths might have length 
zero, and in this case, also the arrows $\alpha_{1}$ and $\alpha_{2}$ might not be present. The following figure is used throughout the proof:

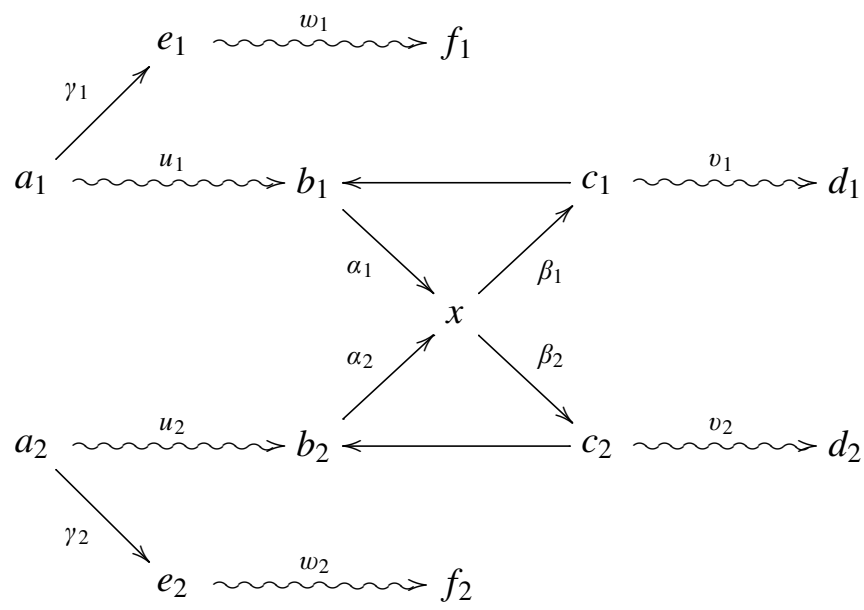

Note that $\left\{x, c_{1}, b_{1}\right\}$ and $\left\{x, c_{2}, b_{2}\right\}$ form oriented cycles in $Q$ such that the composition of any two consecutive arrows is zero. Let

$$
p_{0}: P(0) \rightarrow I_{x}
$$

be a projective cover; then

$$
P(0)=M\left(w_{1}^{-1} \gamma_{1}^{-1} u_{1} \alpha_{1} \beta_{2} v_{2}\right) \oplus M\left(w_{2}^{-1} \gamma_{2}^{-1} u_{2} \alpha_{2} \beta_{1} v_{1}\right)
$$

and

$$
\text { Ker } p_{0}=M\left(w_{1}\right) \oplus M\left(w_{2}\right) \oplus M\left(v_{1}^{-1} \beta_{1}^{-1} \beta_{2} v_{2}\right)
$$

(note that some summands of the terms of this sequence can be zero). We show that $\operatorname{Ker} p_{0}$ is projective, thus obtaining the desired projective resolution

$$
0 \longrightarrow \operatorname{Ker} p_{0} \longrightarrow P(0) \stackrel{p_{0}}{\longrightarrow} I_{x} \longrightarrow 0 .
$$

In order to see that the first two summands of Ker $p_{0}$ are projective (namely the indecomposable projectives $P_{e_{1}}$ and $P_{e_{2}}$ ), one has to show that there are no other arrows starting at the vertices $e_{1}, e_{2}$. Suppose there is an arrow $\delta_{1}: e_{1} \rightarrow y$ in $Q$. Since the algebra $A$ is gentle, the composition $\gamma_{1} \delta_{1}$ lies in the ideal $I$. The assumption of the lemma guarantees the existence of a cycle $\gamma_{1} \delta_{1} \epsilon_{1}$ such that $\gamma_{1} \delta_{1}, \delta_{1} \epsilon_{1}, \epsilon_{1} \gamma_{1} \in I$. But then the simple $A$-module $S_{y}$ would be a composition factor of $I_{x}$, contradicting the assumption $I_{x}=M\left(u_{1} \alpha_{1} \alpha_{2}^{-1} u_{2}^{-1}\right)$. This shows that $M\left(w_{1}\right)=P_{e_{1}}$, and a similar argument shows that $M\left(w_{2}\right)=P_{e_{2}}$. Since $M\left(v_{1}^{-1} \beta_{1}^{-1} \beta_{2} v_{2}\right)=P_{x}$, we conclude that Ker $p_{0}$ is projective.

Example. We illustrate the projective resolution constructed in Lemma 2.6 when $\Gamma$ is the following triangulation of a polygon with 11 vertices (where the midpoints 
of internal arcs are labeled):

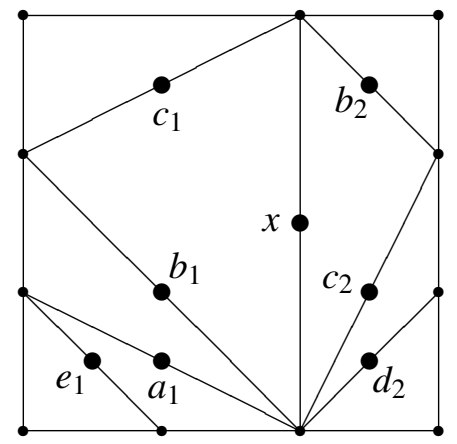

The corresponding algebra $A(\Gamma)$ is given by the quiver

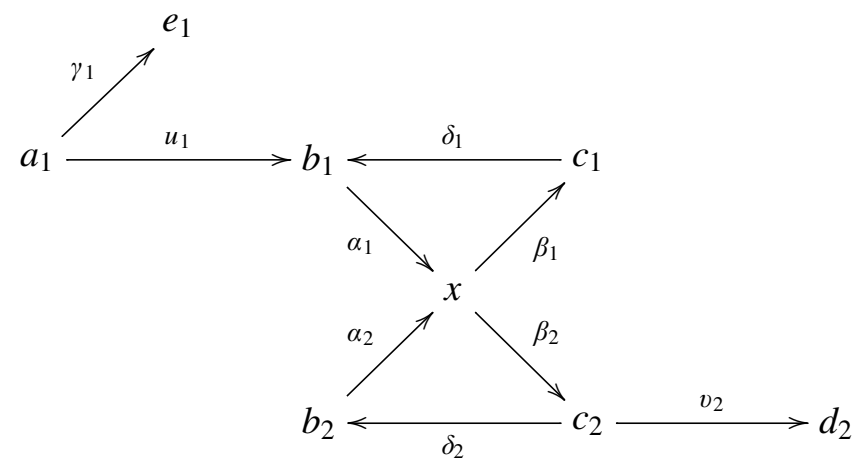

with relations $\alpha_{1} \beta_{1}=\beta_{1} \delta_{1}=\delta_{1} \alpha_{1}=0$ and $\alpha_{2} \beta_{2}=\beta_{2} \delta_{2}=\delta_{2} \alpha_{2}=0$. The projective resolution of the injective module $I_{x}$ is then

$$
0 \longrightarrow P_{e_{1}} \oplus P_{x} \longrightarrow P_{a_{1}} \oplus P_{b_{2}} \longrightarrow I_{x} \longrightarrow 0,
$$

where $P_{e_{1}}$ is simple and $P_{x}=M\left(\beta_{1}^{-1} \beta_{2} v_{2}\right), P_{a_{1}}=M\left(u_{1} \alpha_{1} \beta_{2} v_{2}\right)$ and $P_{b_{2}}=$ $M\left(\alpha_{2} \beta_{1}\right)$.

We recall from [Gabriel 1981] the concept of Galois coverings of bound quiver algebras: Let $\Lambda=k \tilde{Q} / \tilde{I}$ be a bound quiver algebra (where the quiver $\tilde{Q}$ is not necessarily finite). A group $G$ of $k$-linear automorphisms of $\Lambda$ is acting freely on $\Lambda$ if $g e_{x} \neq e_{x}$ for each vertex $x$ of $\tilde{Q}$ and each $g \neq 1$ in $G$. In this case the multiplication in $\Lambda$ induces a multiplication on the set $\Lambda / G$ of $G$-orbits which turns $\Lambda / G$ into an algebra. The canonical projection $\Lambda \rightarrow \Lambda / G$ is called the Galois covering of $\Lambda / G$ with group $G$.

In the following theorem we call (as in [Buan and Vatne 2008]) a 3-cycle an oriented cycle $\alpha \beta \gamma$ where $\alpha, \beta, \gamma$ are three distinct arrows; and by a 3-cycle with radical square zero we mean a 3-cycle $\alpha \beta \gamma$ in an algebra $k Q / I$ such that $\alpha \beta, \beta \gamma, \gamma \alpha$ 
all lie in $I$. By a simple cycle we refer to a subquiver $C$ of $Q$ with $n$ distinct vertices $\left\{x_{0}, x_{1}, \ldots, x_{n-1}, x_{n}=x_{0}\right\}$ and $n$ arrows $\alpha_{i}: x_{i} \rightarrow x_{i+1}$, for $i=1, \ldots, n-1$.

Theorem 2.7. Let $\Gamma$ be a triangulation of an unpunctured marked surface $(S, M)$.

(1) The algebra $A(\Gamma)$ is a gentle algebra.

(2) The algebra $A(\Gamma)$ is Gorenstein of dimension one.

(3) There is a relation in $A(\Gamma)$ from $x$ to $y$ only if there is an arrow $y \rightarrow x$.

(4) $A(\Gamma)$ admits a Galois covering by a bound quiver algebra $k \tilde{Q} / \tilde{I}$ satisfying:

(T1) Every simple cycle in $\tilde{Q}$ is a 3-cycle with radical square zero.

(T2) The only relations in $\tilde{I}$ are those in the 3-cycles.

Proof. Part (1) is shown in Lemma 2.5, and part (2) is shown in Lemma 2.6 since the condition imposed on the gentle algebra $A$ there clearly holds for the algebra $A(\Gamma)$. Part (3) follows directly from the definition of $A(\Gamma)$. Maybe the most intuitive way to obtain the Galois covering required in part (4) is the following. By construction, the only relations in the algebra $A(\Gamma)$ are those in the 3-cycles. In a first step, we identify all 3-cycles to points, replacing each 3-cycle $C$ with vertices $\left\{x_{1}, x_{2}, x_{3}\right\}$ by one single vertex $x$ and replacing each arrow $y \rightarrow x_{i}$ (or $x_{i} \rightarrow y$, respectively) by an arrow $y \rightarrow x$ (or $x \rightarrow y$, respectively). The quiver $\bar{Q}$ thus obtained contains no relations, and we let $\widetilde{\bar{Q}}$ be its universal Galois covering, a (maybe infinite) tree. The bound quiver $(\tilde{Q}, \tilde{I})$ is then obtained by placing back the 3 -cycles $C=\left\{x_{1}, x_{2}, x_{3}\right\}$ for all contracted vertices $x$ of $\widetilde{\bar{Q}}$.

Note that the finite quivers satisfying conditions (T1) and (T2) from the previous theorem form precisely the class of quivers $2_{n}$ considered in [Buan and Vatne 2008], where also the same relations are imposed. It would be interesting to relate the Galois covering $(\tilde{Q}, \tilde{I})$ constructed above with the universal cover of the bordered surface $(S, M)$.

Recovering topological data from $\boldsymbol{A}(\boldsymbol{\Gamma})$. The condition (4) in Theorem 2.7 is very strong. Combined with the fact that the algebra is gentle, it implies the remaining conditions (2) and (3). We show in this section that a gentle algebra satisfying condition (4) is given by an unpunctured marked surface.

First we give a different combinatorial description of the algebras studied here. Consider the following two bound quivers, where type $I$ is a quiver of type $\mathbb{A}_{2}$, and type II is a 3-cycle with radical square zero:

\section{Type I $\longrightarrow$ Type II}

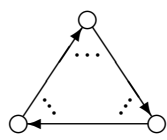

Using these bound quivers one can construct algebras in the following way. Suppose we start with a collection $C$ of disjoint blocks of types I and II. Choose 
a partial matching (that is to say a partial bijection) $\pi$ of the vertices in $C$, where matching a vertex to another vertex of the same block is not allowed. Identifying (or "gluing") the vertices within each pair of the matching we obtain an algebra $A(C, \pi)$. Note that the arrows are not identified by this procedure, so one might obtain parallel arrows or two-cycles. We consider only matchings where the algebra $A(C, \pi)$ is connected.

The procedure of gluing blocks is considered in a more general situation (using plenty of building blocks) in [Brüstle 2001], where the resulting algebras are called kit algebras. A similar construction to glue blocks of type I, II and four more types is described in [Fomin et al. 2008].

We show below that the gentle algebras that admit a Galois covering satisfying conditions (T1) and (T2) from Theorem 2.7 are algebras of the form $A(C, \pi)$, and thus results from [Fomin et al. 2008] concerning these algebras can be applied.

Proposition 2.8. Let $A=k Q / I$ be a gentle algebra where every relation lies on a 3-cycle with radical square zero. Then there exists an unpunctured marked surface $(S, M)$ with a triangulation $\Gamma$ such that $A(\Gamma)=A$.

Proof. The statement follows from [Fomin et al. 2008, (14.1)] once we show that the algebra $A$ admits a unique block decomposition $A=A(C, \pi)$ using blocks of types I and II. We therefore define $C$ to be the disjoint union of all 3-cycles with radical square zero of $A$ together with the disjoint union of all remaining arrows from $A$. Denote by $f$ the quiver morphism $f: C \rightarrow Q$ that identifies the blocks of $C$ with their images in $Q$.

We first show that $\left|f^{-1}(x)\right| \leq 2$ for each vertex $x \in Q$. Indeed, if $f^{-1}(x)$ contains three different vertices, then there are three different arrows in $Q$ adjacent to the vertex $x$. But since the algebra $A$ is gentle, there has to be one relation between these three arrows. However, the set $C$ is constructed in such a way that all relations of $A$ belong to one of the components in $C$, so there are no relations between arrows corresponding to different components of $C$, and so the fiber $f^{-1}(x)$ contains at most two vertices.

We now define a matching $\pi$ on $C$ relating $x_{1}$ to $x_{2}$ whenever $f^{-1}(x)=\left\{x_{1}, x_{2}\right\}$. As required in the definition of $A(C, \pi)$, we do not match a vertex to itself or to some vertex in the same block. It is clear from the construction that $A=A(C, \pi)$. Moreover, the choice of blocks of type I or II is unique since all relations have to correspond to a block of type II.

We would like to point out that all algebras $A(\Gamma)$ given by a triangulation $\Gamma$ of an unpunctured marked surface are of the form $A(C, \pi)$ for some $C$ and $\pi$, but the converse is not true: One can easily produce two-cycles in an algebra $A(C, \pi)$, but this never occurs for the algebras $A(\Gamma)$ as we have shown in Lemma 2.2. 


\section{Gentle cluster-tilted algebras}

Cluster-tilted algebras. Let $\Delta$ be an acyclic quiver. In [Buan et al. 2006] the cluster category $\mathscr{C}_{\Delta}$ is studied in order to obtain a categorical interpretation of the cluster variables of the cluster algebra associated with $\Delta$. It is shown there that clusters correspond bijectively to tilting objects $T$ in $\mathscr{C}_{\Delta}$. Their endomorphism rings $\operatorname{End}_{C_{\Delta}}(T)$ are called cluster-tilted algebras of type $\Delta$. They were introduced and studied in [Buan et al. 2007].

Here we use a different description that has been given in [Assem et al. 2008a]. Let $A$ be the hereditary algebra $k \Delta$. An $A$-module $T$ is called a tilting module if $\operatorname{Ext}_{A}^{1}(T, T)=0$ and the number of isomorphism classes of indecomposable summands of $T$ equals the number of isomorphism classes of simple $A$-modules. In this case, the endomorphism ring $\operatorname{End}_{A}(T)$ is called a tilted algebra of type $\Delta$.

Let $C$ be an algebra of global dimension two. The trivial extension

$$
\tilde{C}=C \ltimes \operatorname{Ext}_{C}^{2}(D C, C)
$$

of $C$ by the $C$ - $C$-bimodule $\operatorname{Ext}_{C}^{2}(D C, C)$ is called the relation-extension of $C$. It is useful to describe explicitly the operations on $\tilde{C}$. As an abelian group, $\tilde{C}=$ $C \oplus \operatorname{Ext}_{C}^{2}(D C, C)$. Therefore, let $(c, \boldsymbol{e})$ and $\left(c^{\prime}, \boldsymbol{e}^{\prime}\right)$ be two elements of $\tilde{C}$, where $\boldsymbol{e}$ and $\boldsymbol{e}^{\prime}$ are respectively represented by the exact sequences of $C$-modules

$$
\begin{array}{ll}
\boldsymbol{e}: & 0 \longrightarrow P \longrightarrow M \longrightarrow N \longrightarrow{ }^{\longrightarrow} \longrightarrow{ }^{\longrightarrow} \longrightarrow{ }^{\prime}, \\
\boldsymbol{e}^{\prime}: & 0 \longrightarrow M^{\prime} \longrightarrow N^{\prime} \longrightarrow I^{\prime} \longrightarrow 0,
\end{array}
$$

with $P, P^{\prime}$ projective and $I, I^{\prime}$ injective. The addition is given by

$$
(c, \boldsymbol{e})+\left(c^{\prime}, \boldsymbol{e}^{\prime}\right)=\left(c+c^{\prime}, \boldsymbol{e}+\boldsymbol{e}^{\prime}\right),
$$

where the sum $c+c^{\prime}$ is the ordinary sum inside $C$, while $\boldsymbol{e}+\boldsymbol{e}^{\prime}$ is the Baer sum in $\operatorname{Ext}_{C}^{2}(D C, C)$ (for which we refer to any textbook of homological algebra). The product in $\tilde{C}$ is given by the formula

$$
(c, \boldsymbol{e})\left(c^{\prime}, \boldsymbol{e}^{\prime}\right)=\left(c c^{\prime}, c \boldsymbol{e}^{\prime}+\boldsymbol{e} c^{\prime}\right),
$$

where the product $c c^{\prime}$ is the ordinary product inside $C$, while $c \boldsymbol{e}^{\prime}$ and $\boldsymbol{e}^{\prime} c$ are defined as follows. Viewing $c \in C$ as an element of End $C_{C} \cong C$, then $\boldsymbol{e}_{1}=c \boldsymbol{e}^{\prime}$ is represented by the sequence obtained by pulling down the sequence $\boldsymbol{e}^{\prime}$ :

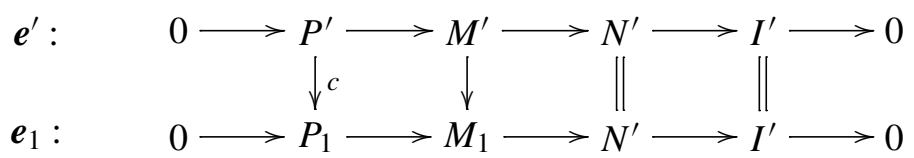

Similarly, if we view $c^{\prime} \in C$ as an element of End $D C_{C} \cong C$, then $\boldsymbol{e}_{2}=\boldsymbol{e} c^{\prime}$ is represented by the sequence obtained by lifting the sequence $\boldsymbol{e}$ : 


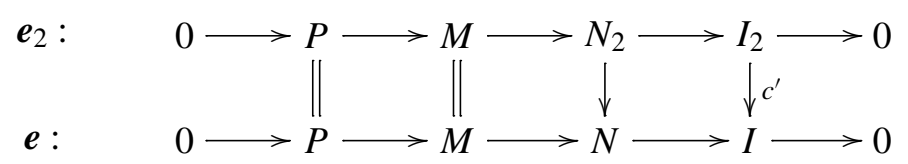

The following theorem allows us to view cluster-tilted algebras as relationextensions of tilted algebras.

Theorem 3.1 [Assem et al. 2008a]. An algebra $\Lambda$ is cluster-tilted of type $\Delta$ if and only if there exists a tilted algebra $C$ of type $\Delta$ such that $\Lambda$ is isomorphic to the relation-extension $\tilde{C}$ of $C$.

Every cluster-tilted algebra satisfies conditions (2) and (3) from Theorem 2.7 [Keller and Reiten 2007]. The bound quivers of cluster-tilted algebras of type $\mathbb{A}$ are explicitly described in [Buan and Vatne 2008, Proposition 3.1]. In fact they were already described in [Caldero et al. 2006] as the algebras $A(\Gamma)$ arising from a triangulation of an unpunctured polygon. The following proposition is contained in [Buan and Vatne 2008], but it also follows from Theorem 3.3 below.

Proposition 3.2 [Buan and Vatne 2008, (3.1)]. An algebra A is cluster-tilted of type $\mathbb{A}$ precisely when $A$ is gentle and there is a presentation $A=k Q / I$ which satisfies conditions (T1) and (T2) from Theorem 2.7.

In particular, the cluster-tilted algebras of type $\mathbb{A}$ are gentle. We describe in the following theorem, whose proof occupies the rest of the section, which of the gentle algebras are cluster-tilted:

Theorem 3.3. Let $C=k Q_{C} / I_{C}$ be a tilted algebra, and $\tilde{C}$ be its relation-extension. The following are equivalent.

(1) $C$ is gentle.

(2) $C$ is tilted of type $\mathbb{A}$ or $\tilde{A}$.

(3) $\tilde{C}$ is gentle.

(4) $\tilde{C}$ is cluster-tilted of type $\mathbb{A}$ or $\tilde{\mathbb{A}}$.

A preliminary part of the proof follows from a result in [Schröer 1999], which says that the class of gentle algebras is stable under tilting.

Lemma 3.4. If a tilted algebra is gentle, then it is tilted of type $\mathbb{A}$ or $\tilde{\mathbb{A}}$.

Proof. Let $\Delta$ be a quiver such that $C$ is tilted of type $\Delta$. Then there exists a tilting $C$-module $T$ such that End $T=k \Delta$. According to [Schröer 1999], $k \Delta$ is a gentle algebra. This implies that the quiver $\Delta$ is of type $\mathbb{A}$ or $\tilde{\mathbb{A}}$.

Lemma 3.5. If $\tilde{C}$ is gentle, then so is $C$.

Proof. This follows from the fact that $\tilde{C}$ is a split extension of $C$ and from [Assem et al. 2008b, (2.7)]. 
Lemma 3.6. The algebra $C$ is tilted of type $\mathbb{A}$ or $\tilde{\mathbb{A}}$ if and only if $\tilde{C}$ is cluster-tilted of type $\mathbb{A}$ or $\tilde{A}$.

Proof. Clearly, if $C$ is tilted of type $\mathbb{A}$ or $\tilde{A}$, then $\tilde{C}$ is cluster-tilted of type $\mathbb{A}$ or $\tilde{\mathbb{A}}$. Conversely, suppose $\tilde{C}$ is cluster-tilted of type $\mathbb{A}$ or $\tilde{\mathbb{A}}$. By [Assem et al. 2008a] there exists a local slice $\Sigma^{\prime}$ in $\bmod \tilde{C}$ such that $C^{\prime}=\tilde{C} /$ Ann $\Sigma^{\prime}$ is tilted of type $\mathbb{A}$ or $\tilde{\mathbb{A}}$. On the other hand, since $\tilde{C}=C \ltimes \operatorname{Ext}_{C}^{2}(D C, C)$, then there exists a local slice $\Sigma$ in $\bmod \tilde{C}$ such that $C=\tilde{C} / \operatorname{Ann} \Sigma$. Since both $\Sigma$ and $\Sigma^{\prime}$ have the same underlying graph, $C$ and $C^{\prime}$ have the same type, so $C$ is tilted of type $\mathbb{A}$ or $\tilde{\mathbb{A}}$.

The main part of the proof of Theorem 3.3 is concerned with the problem of showing that $\tilde{C}$ is gentle if $C$ is tilted of type $\mathbb{A}$ or $\tilde{A}$. This will be done next.

Relation-extensions of tilted algebras of types $\mathbb{A}$ and $\tilde{\mathbb{A}}$. Suppose $C=k Q_{C} / I_{C}$ is tilted of type $\mathbb{A}$ or $\tilde{\mathbb{A}}$. In particular, $C$ is gentle because of [Assem 1982] and [Assem and Skowroński 1987]. Moreover, the quiver of $\tilde{C}$ is known, as are some of its relations, namely those already in $C$ [Assem et al. 2008a; Assem et al. 2008b]. The aim here is to study the remaining relations of $\tilde{C}$.

First, the bound quiver of a tilted algebra of type $\mathbb{A}$ has been described in [Assem 1982], and that of a tilted algebra of type $\tilde{A}$ in [Roldán 1983]. The criterion given here is derived from [Huard and Liu 2000].

We recall that a double-zero in a gentle algebra is a reduced walk of the form $\alpha \beta \omega \gamma \delta$, where $\alpha, \beta, \gamma$ and $\delta$ are arrows such that $\alpha \beta$ and $\gamma \delta$ are relations, while $\omega$ is a nonzero reduced walk (that is, a walk which does not contain any relation). Note that $\omega$ may be trivial and that in this case $\beta$ and $\gamma$ may coincide.

Example. The algebra

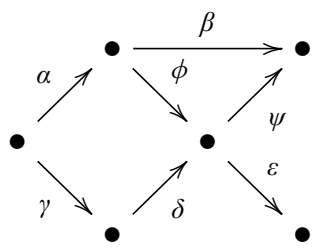

where $\alpha \beta=\phi \psi=\delta \varepsilon=0$, is gentle with a double-zero (namely $\phi \psi \beta^{-1} \phi \psi$ ).

Proposition 3.7 [Assem 1982; Assem and Skowroński 1987]. (1) An algebra is tilted of type $\mathbb{A}$ if and only if it admits a bound quiver presentation $k Q / I$, with $(Q, I)$ a gentle tree with no double-zero.

(2) An algebra is tilted of type $\tilde{A}$ if and only if it admits a bound quiver presentation $k Q / I$, with $(Q, I)$ a gentle presentation with no double-zero and a unique (nonoriented) cycle such that, if the cycle is a band, then all arrows attached to the cycle either enter it or leave it. 
Example. Consider the algebras given by the bound quivers
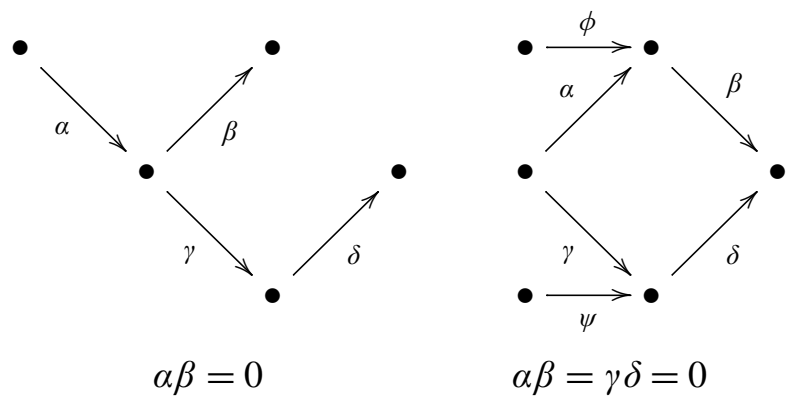

$$
\alpha \beta=0 \quad \alpha \beta=\gamma \delta=0
$$

Using Proposition 3.7, we see that the first one is tilted of type $\mathbb{A}$, while the second one is tilted of type $\tilde{\mathbb{A}}$.

A vanishing criterion. We need a criterion to verify whether a given exact sequence represents the zero element in the second extension group:

Lemma 3.8 [Happel et al. 1996, (II.1.3)]. Given a morphism $f: M \longrightarrow N$, the exact sequence

$$
0 \longrightarrow \operatorname{Ker} f \longrightarrow M \stackrel{f}{\longrightarrow} N \longrightarrow \text { Coker } f \longrightarrow 0
$$

represents the zero element of $\operatorname{Ext}^{2}(\operatorname{Coker} f, \operatorname{Ker} f)$ if and only if there exist a module $X$ and morphisms $g$, $h$ such that the sequence

$$
0 \longrightarrow M \stackrel{(p, g)^{t}}{\longrightarrow} \operatorname{Im} f \oplus X \stackrel{(j, h)}{\longrightarrow} N \longrightarrow 0
$$

is exact, where $p$ and $j$ are the natural morphisms arising from $f$.

The following lemma will be used frequently.

Lemma 3.9. Let $(Q, I)$ be a gentle presentation of an algebra $C$, and let $\alpha: c \longrightarrow b$ and $\beta: b \longrightarrow a$ be arrows in $Q$. Let $\sigma$ and $\eta$ be strings, not passing through $b$, such that $\beta \sigma$ and $\eta \alpha$ are strings. Let $f: M(\beta \sigma) \longrightarrow M(\eta \alpha)$ be a morphism such that $\operatorname{Im} f=S_{b}$.

Then the exact sequence

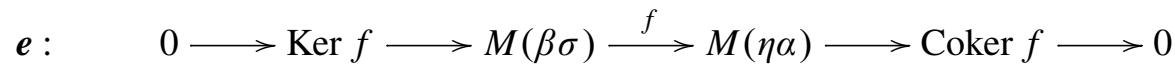

represents a nonzero element of $\operatorname{Ext}_{C}^{2}(\operatorname{Coker} f, \operatorname{Ker} f)$ if and only if $\alpha \beta$ lies in I.

Proof. In view of Lemma 3.8, the sequence $\boldsymbol{e}$ represents a nonzero element of $\operatorname{Ext}_{C}^{2}(\operatorname{Coker} f, \operatorname{Ker} f)$ if and only if there exists no short exact sequence of the form

$$
0 \longrightarrow M(\beta \sigma) \stackrel{(p, g)^{t}}{\longrightarrow} \operatorname{Im} f \oplus X \stackrel{(j, h)}{\longrightarrow} M(\eta \alpha) \longrightarrow 0,
$$


Assume such a sequence exists. Since $S_{b}$ appears exactly once as a composition factor of $M(\beta \sigma)$ and $M(\eta \alpha)$, then it also appears exactly once as a composition factor of $X$. Therefore, there exists a unique indecomposable summand $Y$ of $X$ admitting $S_{b}$ as a composition factor.

We claim that $g: M(\beta \sigma) \longrightarrow X$ is a monomorphism: let $x \in Q_{0}$ and take a vector $v \in M(\beta \sigma)_{x}$ such that $g_{x}(v)=0$. If $x \neq b$, then $p_{x}(v)=0$ and so $(p, g)_{x}^{t}(v)=0$ which implies $v=0$. If $x=b$, then $\left(p_{a}(\beta v), g_{a}\left(M(\beta \sigma)_{\beta}(v)\right)\right)^{t}=$ $(p, g)_{a}^{t}\left(M(\beta \sigma)_{\beta}(v)\right)=(\operatorname{Im} f \oplus X)_{\beta}(p, g)_{b}^{t}(v)=0$ which implies $M(\beta \sigma)_{\beta}(v)=0$. Since $\left(M(\beta \sigma)_{\beta}\right.$ is injective, then $v=0$. This completes the proof of our claim.

Since the evaluation $M(\beta \sigma)_{\beta}$ of the module $M(\beta \sigma)$ on the arrow $\beta$ is nonzero, we must have $X_{\beta} \neq 0$. Now, $S_{b}$ is a composition factor of $Y$, hence $Y_{\beta} \neq 0$ as well. Similarly, $h$ is an epimorphism and it follows that $Y_{\alpha} \neq 0$. On the other hand, $Y$ must be a string or a band module. The above reasoning implies that $\alpha \beta$ must then be a subpath of a string or a band, which implies that $\alpha \beta \notin I$, as required.

Conversely, if $\alpha \beta \notin I$, then we have a short exact sequence

$$
0 \longrightarrow M(\beta \sigma) \longrightarrow S_{b} \oplus M(\eta \alpha \beta \sigma) \longrightarrow M(\eta \alpha) \longrightarrow 0
$$

and hence $\boldsymbol{e}$ represents the zero element in $\operatorname{Ext}_{C}^{2}(\operatorname{Coker} f, \operatorname{Ker} f)$.

Arrows. From now on, let $C$ be a tilted algebra of type $\mathbb{A}$ or $\tilde{A}$. We give a description of the elements of $\tilde{C}=C \ltimes \operatorname{Ext}_{C}^{2}(D C, C)$ corresponding to the arrows of its ordinary quiver. In [Assem et al. 2008a, (2.4)], it is proved that the quiver of $\tilde{C}$ is obtained from that of $C$ by adding an arrow from $x$ to $y$ for each relation from $y$ to $x$. The elements of $\tilde{C}$ corresponding to the arrows of $C$ are of the form $(\alpha, 0)$, where $\alpha$ is an arrow of $C$.

The other arrows correspond to relations in $C$. Let $\alpha \beta$ be a relation from $c$ to $a$ in $C$, and let $\xi_{\alpha \beta}$ be the corresponding new arrow in $\tilde{C}$.

Lemma 3.10. The new arrow $\xi_{\alpha \beta}$ lies in $0 \oplus \operatorname{Ext}_{C}^{2}\left(I_{c}, P_{a}\right)$.

Proof. This new arrow lies in $e_{a} \tilde{C} e_{c}$, which can be written as the direct sum of $e_{a} C e_{c}$ and $e_{a} \operatorname{Ext}_{C}^{2}(D C, C) e_{c}$. We know from Proposition 3.7 that the quiver of $C$ contains no double-zero. Consequently, there are no paths from $a$ to $c$, and hence $e_{a} C e_{c}=0$. Moreover, $e_{a} \operatorname{Ext}_{C}^{2}(D C, C) e_{c}=\operatorname{Ext}_{C}^{2}\left(I_{c}, P_{a}\right)$. The element $\xi_{\alpha \beta}$ thus lies in $0 \oplus \operatorname{Ext}_{C}^{2}\left(I_{c}, P_{a}\right)$.

The following lemma gives the dimension and a basis of the extension space involved in the last expression.

Lemma 3.11. Let $\alpha: c \longrightarrow b$ and $\beta: b \longrightarrow a$ be two arrows of $C$ such that $\alpha \beta \in I_{C}$. 
(a) The dimension of the vector space $\operatorname{Ext}_{C}^{2}\left(I_{c}, P_{a}\right)$ is 1 or 2 . Its dimension is 2 if and only if the following situation occurs in the bound quiver of $C$ :

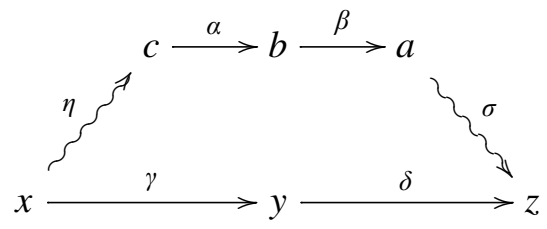

where $\gamma$ and $\delta$ are arrows, $\eta$ and $\sigma$ are paths, possibly stationary, without relations, and $\alpha \beta, \gamma \delta$ are relations.

(b) If the dimension of the space is 1 , then a basis is given by the sequence

$$
\boldsymbol{e}_{1}: \quad 0 \longrightarrow P_{a} \longrightarrow M(\beta \sigma) \longrightarrow M(\eta \alpha) \longrightarrow I_{c} \longrightarrow 0,
$$

where $\eta$ and $\sigma$ are paths such that $I_{c}=M(\eta)$ and $P_{a}=M(\sigma)$.

(c) If the dimension of the space is 2 , then a basis is given by the sequences

$$
\begin{array}{ll}
\boldsymbol{e}_{1}: & 0 \longrightarrow P_{a} \longrightarrow M(\beta \sigma) \longrightarrow M(\eta \alpha) \longrightarrow I_{c} \longrightarrow 0, \\
\boldsymbol{e}_{2}: & \left.0 \longrightarrow P_{a} \longrightarrow M\left(\sigma \delta^{-1}\right) \longrightarrow \gamma^{-1} \eta\right) \longrightarrow I_{c} \longrightarrow 0,
\end{array}
$$

where $\gamma, \delta, \eta$ and $\sigma$ are as in the figure in part (a).

Proof. (a) It is known from [Assem et al. 2008a] that there is a new arrow from $a$ to $c$; thus the dimension cannot be zero. On the other hand, since $C$ is gentle and without double-zero, the local situation of the relation $\alpha \beta$ can be described by the following figure, where dotted lines represent relations.

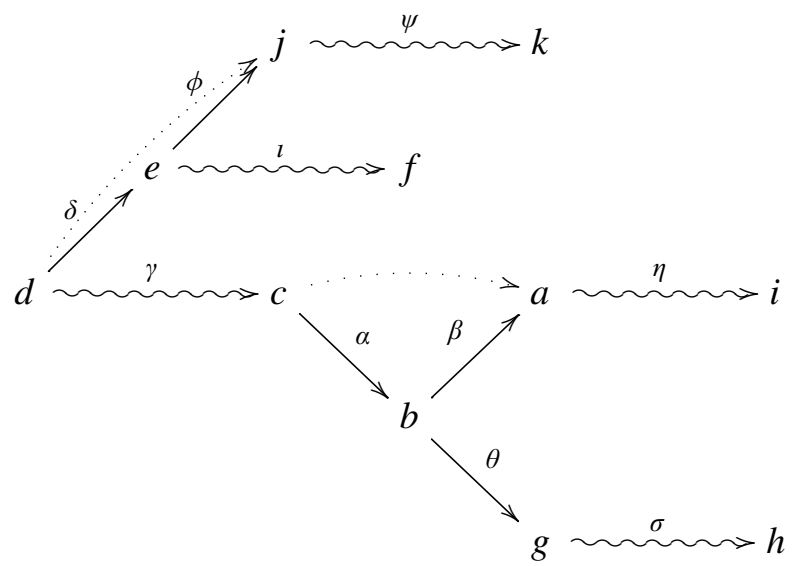

This diagram allows us to compute a projective resolution of $I_{C}$ in $\bmod C$ :

$$
0 \stackrel{p_{3}}{\longrightarrow} P(2) \stackrel{p_{2}}{\longrightarrow} P(1) \stackrel{p_{1}}{\longrightarrow} P(0) \stackrel{p_{0}}{\longrightarrow} I_{c} \longrightarrow 0,
$$


where $P(2)=M(\psi) \oplus M(\eta), P(1)=M\left(l^{-1} \phi \psi\right) \oplus M\left(\sigma^{-1} \theta^{-1} \beta \eta\right)$ and $P(0)=$ $M\left(l^{-1} \delta^{-1} \gamma \alpha \theta \sigma\right)$. Note that some direct summands of the terms of this sequence can be zero. Applying $\operatorname{Hom}_{C}\left(-, P_{a}\right)$, we get a complex

$$
\begin{aligned}
0 \longrightarrow \operatorname{Hom}_{C}\left(I_{c}, P_{a}\right) & \stackrel{\left(p_{0}, P_{a}\right)}{\longrightarrow} \operatorname{Hom}_{C}\left(P(0), P_{a}\right) \\
& \stackrel{\left(p_{1}, P_{a}\right)}{\longrightarrow} \operatorname{Hom}_{C}\left(P(1), P_{a}\right) \stackrel{\left(p_{2}, P_{a}\right)}{\longrightarrow} \operatorname{Hom}_{C}\left(P(2), P_{a}\right) \stackrel{\left(p_{3}, P_{a}\right)}{\longrightarrow} 0 .
\end{aligned}
$$

This yields

$$
\operatorname{Ext}_{C}^{2}\left(I_{c}, P_{a}\right)=\frac{\operatorname{Ker} \operatorname{Hom}\left(p_{3}, P_{a}\right)}{\operatorname{Im} \operatorname{Hom}\left(p_{2}, P_{a}\right)}=\frac{\operatorname{Hom}\left(M(\psi), P_{a}\right) \oplus \operatorname{Hom}\left(M(\eta), P_{a}\right)}{\operatorname{Im} \operatorname{Hom}\left(p_{2}, P_{a}\right)} .
$$

Since $P_{a}=M(\eta)$, we have $\operatorname{dim} \operatorname{Hom}\left(M(\eta), P_{a}\right)=1$, and since

$$
\operatorname{Hom}\left(M\left(\sigma^{-1} \theta^{-1} \beta \eta\right), P_{a}\right)=0,
$$

no nonzero morphism in $\operatorname{Hom}\left(M(\eta), P_{a}\right)$ factors through $p_{2}$.

We claim that $\operatorname{Hom}\left(M(\psi), P_{a}\right)$ is nonzero if and only if $j=i$. Indeed, a nonzero morphism from $M(\psi)$ to $P_{a}$ can only exist when $j$ coincides with a vertex on the path $\eta$. But if $j$ were a vertex different from $i$, then there would be an arrow $\phi^{\prime}: j \rightarrow j^{\prime}$ in the path $\eta$, forcing the relation $\phi \phi^{\prime \prime}$ and creating the double-zero $\delta \phi \phi^{\prime}$. Thus $j=i$. In this case, $\psi$ has no choice but to be the trivial path in $i$, and $\operatorname{dim} \operatorname{Hom}\left(M(\psi), P_{a}\right)=1$. Since $\operatorname{Hom}\left(M\left(l^{-1} \phi \psi, P_{a}\right)=0\right.$, no nonzero morphism in $\operatorname{Hom}\left(M(\psi), P_{a}\right)$ factors through $p_{2}$.

Hence no nonzero morphism in $\operatorname{Hom}\left(M(\psi), P_{a}\right) \oplus \operatorname{Hom}\left(M(\eta), P_{a}\right)$ factors through $p_{2}$. Thus the dimension of this space is either 1 or 2 , and it is 2 exactly when $i=j$. In this case, and in this case only, we have

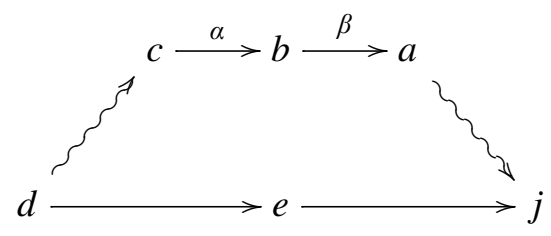

as desired.

(b) It follows from Lemma 3.9 that $\boldsymbol{e}_{1}$ is nonzero. The result follows.

(c) It follows from Lemma 3.9 that $\boldsymbol{e}_{1}$ and $\boldsymbol{e}_{2}$ are nonzero. It remains to be shown that $\boldsymbol{e}_{1}$ and $\boldsymbol{e}_{2}$ are linearly independent. Suppose there exists a nonzero scalar $\lambda$ such that $\boldsymbol{e}_{2}+\lambda \boldsymbol{e}_{1}=0$. Computing this sum, we get the sequence

$$
0 \longrightarrow P_{a} \longrightarrow M\left(\beta \sigma \delta^{-1}\right) \stackrel{f}{\longrightarrow} M\left(\gamma^{-1} \eta \alpha\right) \longrightarrow I_{c} \longrightarrow 0,
$$

where all morphisms are multiples of the natural morphisms between string modules. 
Here, applying Lemma 3.9 is not possible, since $\operatorname{Im} f=S_{b} \oplus S_{y}$, but a similar technique of proof can be used.

Suppose there exist a module $X$ and morphisms $g$ and $h$ such that the sequence

$$
0 \longrightarrow M\left(\beta \sigma \delta^{-1}\right) \stackrel{(p, g)^{t}}{\longrightarrow}\left(S_{b} \oplus S_{y}\right) \oplus X \stackrel{(j, h)}{\longrightarrow} M\left(\gamma^{-1} \eta \alpha\right) \longrightarrow 0
$$

is exact, where $f=j p$ is the canonical factorisation. Since $S_{b}$ appears exactly once as a composition factor of $M\left(\beta \sigma \delta^{-1}\right)$ and $M\left(\gamma^{-1} \eta \alpha\right)$, it also appears exactly once as a composition factor of $X$. Therefore, there exists a unique indecomposable summand $Y$ of $X$ admitting $S_{b}$ as a composition factor. As in the proof of Lemma 3.9, we show that $Y_{\beta} \neq 0$ and $Y_{\alpha} \neq 0$. Therefore, $\alpha \beta$ must be a subpath of a string or a band, which is a contradiction, since it is a relation.

The sequences $\boldsymbol{e}_{1}$ and $\boldsymbol{e}_{2}$ thus form a basis of the extension space.

It remains to determine which of the basis elements are represented by arrows of $\tilde{C}$.

Lemma 3.12. Let $\alpha: c \longrightarrow b$ and $\beta: b \longrightarrow a$ be two arrows of the quiver of $C$ such that $\alpha \beta$ is a relation. Let $\xi_{\alpha \beta}$ be the corresponding new arrow in $\tilde{C}$. With the notation of Lemma 3.11, the element $\left(0, \boldsymbol{e}_{1}\right)$ can be chosen to represent $\xi_{\alpha \beta}$.

Proof. The space $0 \oplus \operatorname{Ext}_{C}^{2}\left(I_{c}, P_{a}\right)$ contains at least one arrow.

If its dimension is 1 , the result is obvious.

If its dimension is 2, Lemma 3.11 describes the situation of $\alpha \beta$ in the quiver of $C$. Two cases arise.

First, suppose that $\eta$ and $\sigma$ are both trivial paths.

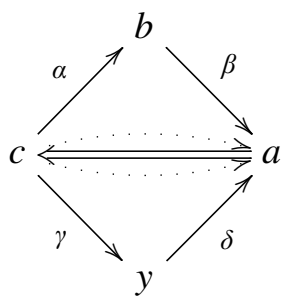

In this case, two arrows from $a$ to $c$ are added to the quiver. Both $\left(0, \boldsymbol{e}_{1}\right)$ and $\left(0, \boldsymbol{e}_{2}\right)$ must thus represent arrows of $\tilde{C}$.

Second, suppose $\eta$ and $\sigma$ are not both trivial. In this case, Lemma 3.11 implies that $\operatorname{Ext}_{C}^{2}\left(I_{x}, P_{z}\right)$ is of dimension 1, and that a basis is given by

$$
\boldsymbol{e}^{\prime}: \quad 0 \longrightarrow P_{z} \longrightarrow M(\delta) \longrightarrow M(\gamma) \longrightarrow I_{x} \longrightarrow 0 .
$$

Reasoning as above, we get that $\left(0, \boldsymbol{e}^{\prime}\right)$ represents the new arrow from $z$ to $x$. Moreover, a straightforward calculation yields $(\sigma, 0)\left(0, \boldsymbol{e}^{\prime}\right)(\eta, 0)=\left(0, \boldsymbol{e}_{2}\right)$. 
Since one of $\eta$ and $\sigma$ is not trivial, one of $(0, \eta)$ and $(0, \sigma)$ must lie in $\operatorname{rad} \tilde{C}$. Therefore $\left(0, \boldsymbol{e}_{2}\right) \in \operatorname{rad}^{2} \tilde{C}$, and $\left(0, \boldsymbol{e}_{1}\right) \in \operatorname{rad} \tilde{C} \backslash \operatorname{rad}^{2} \tilde{C}$; in other words, $\left(0, \boldsymbol{e}_{1}\right)$ represents an arrow from $a$ to $c$.

Relations. Knowing how to write arrows in $\tilde{C}$ allows us to compute the relations.

Lemma 3.13. Let $C=k Q_{C} / I_{C}$ and $\tilde{C}=k Q_{\tilde{C}} / I_{\tilde{C}}$.

(1) Let $\omega_{1}, \omega_{2}, \ldots, \omega_{n}$ be paths from $x$ to $y$ in the quiver of $C$, and let $\lambda_{1}, \lambda_{2}, \ldots$, $\lambda_{n} \in k$. Then $\sum_{i=1}^{n} \lambda_{i}\left(\omega_{i}, 0\right)=0$ in $\tilde{C}$ if and only if $\sum_{i=1}^{n} \lambda_{i} \omega_{i}=0$ in $C$.

(2) Let $\alpha: c \longrightarrow b$ and $\beta: b \longrightarrow a$ be two arrows in the quiver of $C$ such that $\alpha \beta$ is a relation. Let $\left(0, \boldsymbol{e}_{1}\right)$ be the element representing the corresponding new arrow, where $\boldsymbol{e}_{1}$ is as in Lemma 3.11. Then $\left(0, \boldsymbol{e}_{1}\right)(\alpha, 0)=0$ and $(\beta, 0)\left(0, \boldsymbol{e}_{1}\right)=0$.

(3) The ideal $I_{\tilde{C}}$ is generated by the relations of $C$ and those described in (2).

Proof. (1) This is shown in [Assem et al. 2008b].

(2) Viewing $\alpha$ as an element of End $D C$, or more precisely as a morphism from $I_{b}$ to $I_{c}$, we can compute $\boldsymbol{e}_{1} \beta$ :

$$
\boldsymbol{e}_{1} \beta: \quad 0 \longrightarrow P_{a} \longrightarrow M(\beta \sigma) \longrightarrow M(\eta \alpha) \oplus M(\varphi \gamma) \longrightarrow I_{b} \longrightarrow 0,
$$

where $I_{b}=M\left(\eta \alpha \gamma^{-1} \varphi^{-1}\right)$. This sequence represents the zero element, because of Lemma 3.8 and exactness of the sequence

$$
0 \longrightarrow M(\beta \sigma) \longrightarrow S_{b} \oplus M(\varphi \gamma \beta \sigma) \oplus M(\eta \alpha) \longrightarrow M(\varphi \gamma) \oplus M(\eta \alpha) \longrightarrow 0 .
$$

Therefore $\left(0, \boldsymbol{e}_{1}\right)(\alpha, 0)=0$.

In a dual way, we prove that $(\beta, 0)\left(0, \boldsymbol{e}_{1}\right)=0$.

(3) It is sufficient to show that new arrows in the quiver of $\tilde{C}$ are not involved in other relations than those described in (2).

First suppose that $w$ is a monomial relation involving new arrows and relations other than those described in (2). Then it must contain exactly one new arrow $\xi$, corresponding to a relation $\alpha \beta$; otherwise the quiver of $C$ would contain a double-zero. Write $w=u \xi v$, where $u$ and $v$ are nonzero paths consisting of arrows of $C$. Let $\boldsymbol{e}_{1}$ be the sequence as in Lemma 3.11 corresponding to $\xi$. Then $(u, 0)\left(0, \boldsymbol{e}_{1}\right)(v, 0)=\left(0, u \boldsymbol{e}_{1} v\right)$, where $u \boldsymbol{e}_{1} v$ is the sequence

$$
0 \longrightarrow M\left(u^{-1} u^{\prime}\right) \longrightarrow M\left(\beta u^{-1} u^{\prime}\right) \longrightarrow M\left(v^{\prime} v^{-1} \alpha\right) \longrightarrow M\left(v^{\prime} v^{-1}\right) \longrightarrow 0,
$$

where $u^{\prime}$ and $v^{\prime}$ are paths in the quiver of $C$. The figure at the top of the next page illustrates the local situation, where $\alpha \beta=\gamma^{\prime} \delta^{\prime}=0$; the last arrow of $u$ and the first 
of $t$ form a relation, as do the last of $v$ and $v^{\prime}$ and the first of $w^{\prime}$ and $w$, respectively.

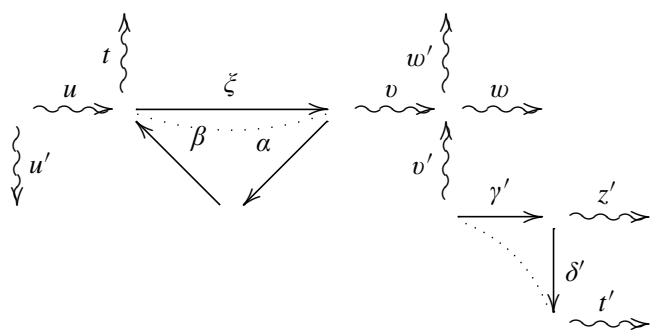

This yields the following commutative diagram, where the first line is a projective resolution of $M\left(v^{\prime} v^{-1}\right)$ :

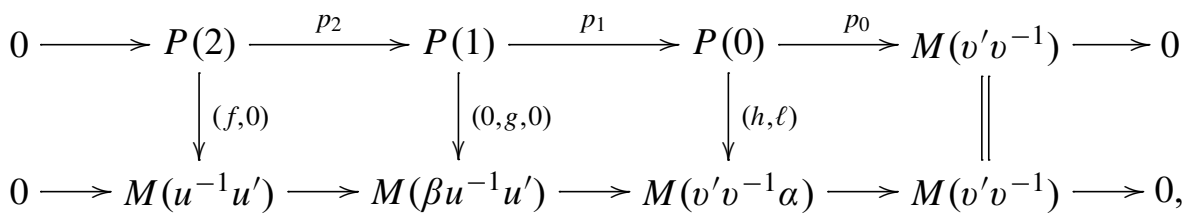

where $P(2)=M(t) \oplus M\left(t^{\prime}\right), P(1)=M\left(w^{\prime} w\right) \oplus M\left(z^{-1} \gamma^{-1} \beta t\right) \oplus M\left(z^{\prime-1} \delta^{\prime} t^{\prime}\right)$ and $P(0)=M\left(z^{-1} \gamma^{-1} \alpha^{-1} v w\right) \oplus M\left(z^{\prime-1} \gamma^{\prime-1} v^{\prime} w^{\prime}\right)$, and all nonzero morphisms are the natural morphisms between string modules. It is then seen that $(f, 0)$ cannot factor through $p_{2}$, and thus the lower exact sequence is nonzero. Hence there are no other monomial relations than those in (2).

Now suppose we have a minimal relation of the form $\sum_{\tilde{C}}^{m} \lambda_{i} w_{i}$, where each $\lambda_{i}$ is a nonzero scalar, each $w_{i}$ is a path in the quiver of $\tilde{C}$, and $m \geq 2$. At least one of the $w_{i}$ must pass through a new arrow, and since $C$ contains no double zero, this implies that each $w_{i}$ must pass through exactly one new arrow, say $\xi_{i}$, corresponding to a relation $\alpha_{i} \beta_{i}$. Write $w_{i}=u_{i} \xi_{i} v_{i}$, where $u_{i}$ and $v_{i}$ are paths of the quiver of $C$.

Since the quiver of $C$ contains at most one cycle, we must have $m=2$. Since $k$ is a field, we may suppose that $\lambda_{1}=1$. Letting $\boldsymbol{e}_{1}$ and $\boldsymbol{e}_{2}$ be the sequences associated to $\xi_{1}$ and $\xi_{2}$, respectively, we get that $u_{1} \boldsymbol{e}_{1} v_{1}$ and $\lambda_{2} u_{2} \boldsymbol{e}_{2} v_{2}$ are both sequences of the form above. Their sum is the sequence

$$
0 \longrightarrow M\left(u_{2}^{-1} u_{1}\right) \longrightarrow M\left(\beta_{2} u_{2}^{-1} u_{1} \beta_{1}^{-1}\right)
$$

$$
\longrightarrow M\left(\alpha_{2}^{-1} v_{2} v_{1}^{-1} \alpha_{1}\right) \longrightarrow M\left(v_{1} v_{2}^{-1}\right) \longrightarrow 0 .
$$

By an argument similar to the one given in the proof of Lemma 3.11(c), this element is not zero; a contradiction. Hence no binomial relations exist in $\tilde{C}$.

The relations described in the preceding lemma make $\tilde{C}$ a gentle algebra.

Lemma 3.14. If $\tilde{C}$ is cluster-tilted of type $\mathbb{A}$ or $\tilde{A}$, then $\tilde{C}$ is gentle. 
Proof. The relations of $\tilde{C}$ are known (see Lemma 3.13). Moreover, $C$ is gentle.

Suppose that there are $r$ new arrows. Let us add the new arrows and the corresponding new relations one by one, thus obtaining a sequence $C=C_{0}, C_{1}, \ldots$, $C_{r}=\tilde{C}$ of algebras. We show that $C_{i}$ is gentle for all $i$ in $\{0,1,2, \ldots, r\}$.

Since $C$ is gentle, then so is $C_{0}$. Suppose that $C_{i}$ is gentle, where $i$ is in $\{0,1,2, \ldots, r-1\}$. To get $C_{i+1}$, we add one new arrow, say $\gamma$ from $x$ to $y$. This arrow comes from a relation $\alpha \beta$ from $y$ to $x$ in $C$. We must add the relations $\beta \gamma$ and $\gamma \alpha$ to obtain $C_{i+1}$.

Since $C_{i}$ is gentle, there were already at most two arrows starting from $x$ in $C_{i}$. Suppose that there were two, say $\eta_{1}$ and $\eta_{2}$. Since $C_{i}$ is gentle, then $\beta$ is involved in a relation with one of the two, say $\eta_{1}$. The arrow $\eta_{1}$ cannot be in $C$, otherwise there would be a double zero involving $\alpha \beta$ and $\beta \eta_{1}$. So the arrow $\eta_{1}$ comes from a relation $\sigma \beta$ in $C$. Since $C$ is gentle, we must have that $\sigma=\alpha$, so that $\eta_{1}=\gamma$, which is absurd because $\gamma$ is not in $C_{i}$.

Therefore, in $C_{i}$, there is at most one outgoing arrow from $x$, and this arrow is not involved in a relation with $\beta$. This shows that in $C_{i+1}$, there are at most two arrows starting from $x$, say $\eta$ and $\gamma$, and that $\beta \eta$ is not a relation while $\beta \gamma$ is. Moreover, there is at most one more arrow ending in $x$, say $\delta$, and since $C_{i}$ is gentle, we have that $\delta \eta$ is a relation, while $\delta \gamma$ is not. So the relations at $x$ are those found in a gentle algebra.

Using a similar argument for the vertex $y$, we get that $C_{i+1}$ is a gentle algebra. By induction, $\tilde{C}$ is a gentle algebra.

Example. Lemma 3.13 allows us to compute the relation-extension of any gentle tilted algebra. As an illustration, consider the two algebras given in the example on page 216. The relation-extension of each is given in the following diagram:
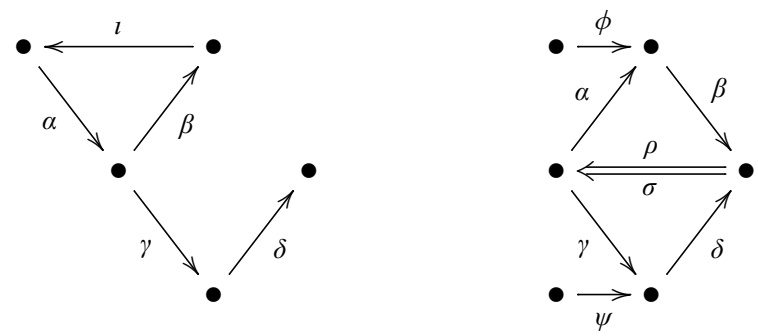

$$
\begin{array}{r}
\alpha \beta=\imath \alpha=\beta \imath=0 \quad \gamma \delta=\rho \alpha=0 \\
\beta \rho=\sigma \gamma=\delta \sigma=0
\end{array}
$$

Proof of the main theorem. Now the proof of Theorem 3.3, developed in separate parts over the last several pages, can be stated properly. 
Proof of Theorem 3.3. That (1) implies (2) is shown in Lemma 3.4, that (2) implies (4) in Lemma 3.6, that (4) implies (3) in Lemma 3.14, and that (3) implies (1) in Lemma 3.5.

\section{Geometry of surfaces and $A(\Gamma)$}

We study in this section more connections between geometric properties of the marked surface $(S, M)$ and properties of the algebra $A(\Gamma)$ given by a triangulation of $(S, M)$.

Cluster-tilted algebras arising from surfaces. We first address the question of which of the algebras $A(\Gamma)$ are cluster-tilted. Recall that all algebras $A(\Gamma)$ share the properties (2) and (3) from Theorem 2.7 with every cluster-tilted algebra. Moreover, it is shown in [Caldero et al. 2006] and [Buan and Vatne 2008] that the cluster-tilted algebras of type $\mathbb{A}$ are algebras $A(\Gamma)$ arising from a triangulation of an unpunctured polygon. In this section, we show the following generalization:

Theorem 4.1. Let $A(\Gamma)$ be the algebra associated to the triangulation $\Gamma$ of an unpunctured marked surface $(S, M)$. Then the following statements are equivalent:

(1) The algebra $A(\Gamma)$ is cluster-tilted.

(2) The algebra $A(\Gamma)$ is cluster-tilted of type $\mathbb{A}$ or $\tilde{\mathbb{A}}$.

(3) $S$ is a disc or an annulus.

Moreover, all cluster-tilted algebras of type $\mathbb{A}($ or $\tilde{\mathbb{A}})$ are of the form $A(\Gamma)$ for some triangulation $\Gamma$ of a disc $S$ (or an annulus $S$, respectively).

Proof. It is clear that (2) implies (1). Let us show the converse: Suppose that the algebra $A(\Gamma)$ is cluster-tilted. Thus there is a sequence of mutations transforming the quiver with potential defining $A(\Gamma)$ into some quiver $Q$ with zero potential. This sequence of mutations corresponds to a sequence of flips, transforming the triangulation $\Gamma$ of $(S, M)$ into a triangulation $T$ with $Q(T)=Q$ and zero potential. Hence $A(T)=k Q$ is hereditary. Since we know from Theorem 2.7 that $A(T)$ is gentle, this leaves only the possibilities that $Q$ is of type $\mathbb{A}$ or $\widetilde{\mathbb{A}}$. Therefore the algebra $A(\Gamma)$ is cluster-tilted of type $\mathbb{A}$ or $\tilde{A}$.

We prove now the equivalence of (2) and (3). Since all triangulations on $(S, M)$ are flip-equivalent [Hatcher 1991] and flips of the triangulation correspond to mutations of the corresponding quiver with potential [Labardini-Fragoso 2009], it is sufficient to consider one particular triangulation. In the case where $S$ is a disc, we choose the triangulation to be in the form of a fan, giving rise to a linear oriented quiver of type $\mathbb{A}$. In the case where $S$ is an annulus, we choose the triangulation given by two fans in opposite direction as shown in the figure at the top of the next page (where the left and right vertical edge should be identified). 


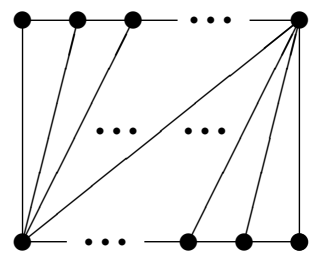

The corresponding quiver is of type $\tilde{\mathbb{A}}$ with zero potential; thus (3) implies (2). Conversely, we know from Proposition 2.8 that the quivers $Q(\Gamma)$ uniquely determine the topology of the unpunctured marked surface $(S, M)$. Therefore $S$ is a disc or an annulus, respectively, and since all triangulations are flip-equivalent, it is clear that all cluster-tilted algebras of the corresponding type occur.

Curves in ( $S, M)$ and string modules. In this section we are comparing strings in $A(\Gamma)$ to curves in $(S, M)$. By a curve in $(S, M)$ we mean a curve $\gamma$ in $S$ whose endpoints lie in $M$ and where all points except the endpoints lie in the interior of $S$. We usually consider curves up to homotopy. For instance, for two distinct curves $\gamma$ and $\delta$ in $(S, M)$, the intersection number $I_{\Gamma}(\gamma, \delta)$ is defined as the minimal number of transversal intersections of two representatives of the homotopy classes of $\gamma$ and $\delta$. Denote the internal arcs of the triangulation $\Gamma$ by $\left\{a_{1}, \ldots, a_{n}\right\}$. Then we define the intersection vector $I_{\Gamma}(\gamma)$ of a curve $\gamma$ as

$$
I_{\Gamma}(\gamma)=\left(I_{\Gamma}\left(\gamma, a_{1}\right) \ldots, I_{\Gamma}\left(\gamma, a_{n}\right)\right) .
$$

Proposition 4.2. Let $\Gamma$ be a triangulation of $(S, M)$, an unpunctured marked surface. Then there exists a bijection $\{\gamma\} \mapsto w(\gamma)$ between the homotopy classes of curves in $(S, M)$ not homotopic to an arc in $\Gamma$ and the strings of $A(\Gamma)$. Under this bijection, the intersection vector corresponds to the dimension vector of the corresponding string module, that is,

$$
I_{\Gamma}(\gamma)=\underline{\operatorname{dim}} M(w(\gamma)) .
$$

Proof. Let $w=x_{1} \stackrel{\alpha_{1}}{\longleftrightarrow} x_{2} \stackrel{\alpha_{2}}{\longleftrightarrow} \cdots \stackrel{\alpha_{s-1}}{\longleftrightarrow} x_{s}$ be a string in $A(\Gamma)$. We define a curve $\gamma(w)$ in $(S, M)$ as follows: The arcs $x_{1}$ and $x_{2}$ belong to the same triangle $T_{1}$ since they are joined by an arrow in $A(\Gamma)$. We connect the midpoints of $x_{1}$ and $x_{2}$ by a curve $\gamma_{1}$ in the interior of $T_{1}$. Proceeding in the same way with the remaining $\operatorname{arcs} x_{2}, \ldots, x_{s}$ we obtain curves $\gamma_{2}, \ldots, \gamma_{s-1}$ connecting the midpoints of the respective arcs. The internal arc $x_{1}$ belongs to two triangles: the triangle $T_{1}$ which we considered above and another triangle $T_{0}$. Let $P \in M$ be the marked point in $T_{0}$ opposite to the arc $x_{1}$. We now connect $P$ with the midpoint of $x_{1}$ by a curve $\gamma_{0}$ in the interior of $T_{0}$, and proceed in the same way on the other end of the string $w$, connecting the midpoint of $x_{s}$ with a marked point $Q$ by some curve $\gamma_{s}$. The curve $\gamma(w)$ is then defined as the concatenation of the curves $\gamma_{0}, \ldots, \gamma_{s}$. 

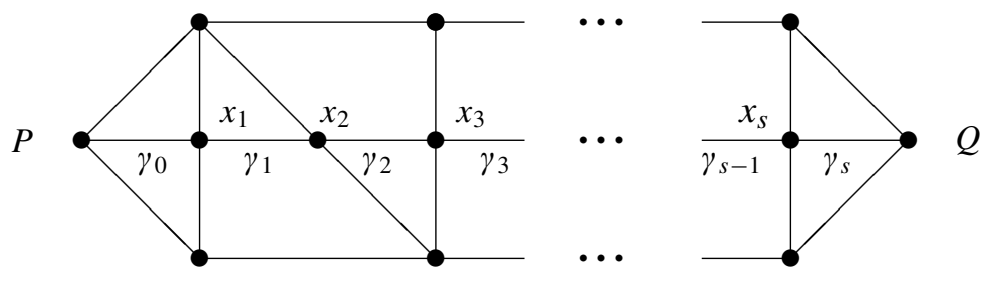

By construction, the points of intersection of the curve $\gamma(w)$ with arcs in $\Gamma$ are indexed by the vertices of the string $w$. The curve intersects the $\operatorname{arcs}$ of $\Gamma$ transversally, and since the string $w$ is reduced, none of the $\gamma_{i}$ is homotopic to a piece of an arc in $\Gamma$. Thus the intersection numbers are minimal, and $I_{\Gamma}(\gamma(w))=$ $\underline{\operatorname{dim}} M(w)$. Since $\gamma(w)$ has nontrivial intersection with arcs of $\Gamma$, it is clear that it is not homotopic to an arc in the triangulation $\Gamma$.

Conversely, let $\gamma:[0,1] \rightarrow S$ be a curve in $(S, M)$ which is not homotopic to an arc in $\Gamma$. We assume that the curve $\gamma$ is chosen (in its homotopy class) such that it intersects the arcs $a$ of $\Gamma$ transversally (if at all) and such that the intersection numbers $I_{\Gamma}(\gamma, a)$ are minimal. Orienting $\gamma$ from $P=\gamma(0) \in M$ to $Q=\gamma(1) \in M$, we denote by $x_{1}$ the first internal arc of $\Gamma$ that intersects $\gamma$, by $x_{2}$ the second arc, and so on. We thus obtain a sequence $x_{1}, \ldots, x_{s}$ of (not necessarily different) internal arcs in $\Gamma$. Since the intersection numbers are minimal, we know that $x_{i} \neq x_{i+1}$. Thus there are arrows, either $\alpha_{i}: x_{i} \rightarrow x_{i+1}$ or $\alpha_{i}: x_{i+1} \rightarrow x_{i}$ in $Q(\Gamma)$, and we obtain a walk $w(\gamma)=x_{1} \stackrel{\alpha_{1}}{\longleftrightarrow} x_{2} \stackrel{\alpha_{1}}{\longleftrightarrow} \cdots \stackrel{\alpha_{s-1}}{\longleftrightarrow} x_{s}$ in $Q(\Gamma)$. The fact that $\gamma$ intersects the arcs of $\Gamma$ transversally implies that the walk $w(\gamma)$ is reduced and avoids the zero-relations, and thus $w(\gamma)$ is a string in $A(\Gamma)$.

It follows from their construction that the two maps between strings and homotopy classes of curves defined above are mutually inverse.

Remark. Recall that two string modules $M(w)$ and $M(v)$ are isomorphic precisely when $v=w$ or $v=w^{-1}$. The inverse string $w^{-1}$ corresponds to orienting the curve in the opposite direction.

Proposition 4.3. Let $\Gamma$ be a triangulation of $(S, M)$, an unpunctured marked surface. Then there exists a bijection between the homotopy classes of closed curves in $(S, M)$ and powers $b^{n}$ of bands $b$ of $A(\Gamma)$.

The proof is analogous to that of the previous proposition.

An example where $A(\Gamma)$ is not cluster-tilted. We finally present in this section an example of an algebra $A(\Gamma)$ which is not cluster-tilted. Recall that an algebra $A$ is tame if for all $d \in \mathbb{N}$ there is a finite number $n_{d}$ of one-parameter families of $A$-modules such that almost every $d$-dimensional $A$-module belongs to one of these $n_{d}$ families. The algebra $A$ is said to be domestic if there is a constant $c$ such that $n_{d} \leq c$ for all $d \in \mathbb{N}$. On the other hand, if the numbers $n_{d}$ grow faster than any polynomial, then the tame algebra $A$ is said to be of nonpolynomial growth. 
It is well-known that every string algebra $A$ is tame, and that the one-parameter families are given by the bands in $A$ [Butler and Ringel 1987]. In particular, all the algebras $A(\Gamma)$ are tame, because they are gentle and thus string algebras. Moreover the tame cluster-tilted algebras of the form $A(\Gamma)$ studied here are all domestic; in fact, they are of type $\mathbb{A}$ or $\tilde{\mathbb{A}}$, and thus we may assume above that $c=0$ or $c=1$. We construct in this section an example of an algebra $A(\Gamma)$ which is of nonpolynomial growth, and thus cannot be cluster-tilted. To obtain this example, we consider a sphere $S$ with three holes and choose one marked point in each boundary component. We fix the following triangulation $\Gamma$ of $(S, M)$ :

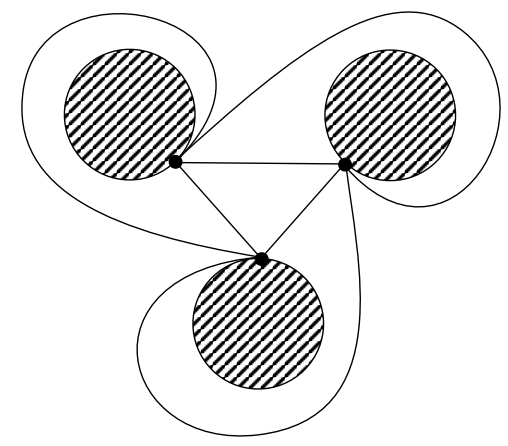

Then the algebra $A(\Gamma)$ is given by the following quiver with relations $\epsilon_{i} \rho_{i}=$ $0, \rho_{i} \sigma_{i}=0$ and $\sigma_{i} \epsilon_{i}=0$ for $i=1$ and $i=2$.

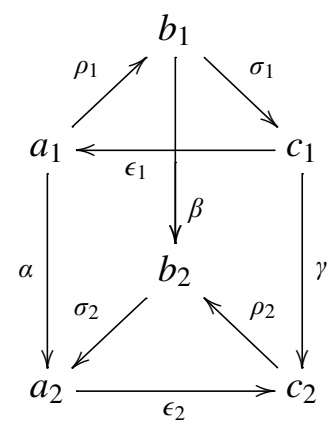

The string algebra $A(\Gamma)$ admits the two bands

$$
\begin{aligned}
& \xi=b_{2} \stackrel{\sigma_{2}}{\longrightarrow} a_{2} \stackrel{\alpha}{\longleftarrow} a_{1} \stackrel{\rho_{1}}{\longrightarrow} b_{1} \stackrel{\beta}{\longrightarrow} b_{2}, \\
& \eta=b_{2} \stackrel{\rho_{2}}{\longleftarrow} c_{2} \stackrel{\gamma}{\longleftarrow} c_{1} \stackrel{\sigma_{1}}{\longleftarrow} b_{1} \stackrel{\beta}{\longrightarrow} b_{2} .
\end{aligned}
$$

Since $\xi$ and $\eta$ can be composed arbitrarily, the number of bands of a fixed length $l$ grows exponentially with $l$, so the algebra $A(\Gamma)$ is of nonpolynomial growth.

We would like to point out that the notion of nonpolynomial growth of tame algebras discussed here does not coincide with the notion of nonpolynomial growth cluster algebras discussed in [Fomin et al. 2008]: There one counts the number of 
cluster variables, that is to say, of $\operatorname{arcs}$ in $(S, M)$, instead of one-parameter families, that is to say, closed curves in $(S, M)$. In [Fomin et al. 2008] the example we are considering in this section is classified as being of polynomial growth, meaning that, even if the number of curves is growing exponentially, the number of arcs is bounded for the sphere with three holes.

\section{References}

[Assem 1982] I. Assem, “Tilted algebras of type $\mathbf{A}_{n}$ ", Comm. Algebra 10:19 (1982), 2121-2139. MR 84c:16028 Zbl 0495.16028

[Assem and Skowroński 1987] I. Assem and A. Skowroński, "Iterated tilted algebras of type $\tilde{\mathbf{A}}_{n}$ ", Math. Z. 195:2 (1987), 269-290. MR 88m:16033 Zbl 0601.16022

[Assem et al. 2006] I. Assem, D. Simson, and A. Skowroński, Elements of the representation theory of associative algebras, Vol. 1: Techniques of representation theory, London Mathematical Society Student Texts 65, Cambridge University Press, 2006. MR 2006j:16020 Zbl 1092.16001

[Assem et al. 2008a] I. Assem, T. Brüstle, and R. Schiffler, "Cluster-tilted algebras as trivial extensions”, Bull. Lond. Math. Soc. 40:1 (2008), 151-162. MR 2009c:16086 Zbl 05267878

[Assem et al. 2008b] I. Assem, F. U. Coelho, and S. Trepode, "The bound quiver of a split extension”, J. Algebra Appl. 7:4 (2008), 405-423. MR 2009g:16022 Zbl 05381819

[Barot et al. 2008] M. Barot, E. Fernández, M. I. Platzeck, N. I. Pratti, and S. Trepode, "From iterated tilted algebras to cluster-tilted algebras", preprint, 2008. arXiv 0811.1328

[Brüstle 2001] T. Brüstle, "Kit algebras", J. Algebra 240:1 (2001), 1-24. MR 2002c:16021 Zbl 0991.16006

[Buan and Vatne 2008] A. B. Buan and D. F. Vatne, "Derived equivalence classification for clustertilted algebras of type $A_{n}$ ", J. Algebra 319:7 (2008), 2723-2738. MR 2009j:16013 Zbl 1155.16010

[Buan et al. 2006] A. B. Buan, R. Marsh, M. Reineke, I. Reiten, and G. Todorov, "Tilting theory and cluster combinatorics", Adv. Math. 204:2 (2006), 572-618. MR 2007f:16033 Zbl 1127.16011

[Buan et al. 2007] A. B. Buan, R. J. Marsh, and I. Reiten, "Cluster-tilted algebras", Trans. Amer. Math. Soc. 359:1 (2007), 323-332. MR 2007f:16035 Zbl 1123.16009

[Butler and Ringel 1987] M. C. R. Butler and C. M. Ringel, "Auslander-Reiten sequences with few middle terms and applications to string algebras", Comm. Algebra 15:1-2 (1987), 145-179. MR 88a:16055 Zbl 0612.16013

[Caldero et al. 2006] P. Caldero, F. Chapoton, and R. Schiffler, "Quivers with relations arising from clusters ( $A_{n}$ case)", Trans. Amer. Math. Soc. 358:3 (2006), 1347-1364. MR 2007a:16025 Zbl 1137.16020

[Derksen et al. 2008] H. Derksen, J. Weyman, and A. Zelevinsky, "Quivers with potentials and their representations, I: Mutations”, Selecta Math. (N.S.) 14:1 (2008), 59-119. MR 2480710 Zbl 05573998

[Fomin and Zelevinsky 2002] S. Fomin and A. Zelevinsky, "Cluster algebras, I: Foundations", J. Amer. Math. Soc. 15:2 (2002), 497-529. MR 2003f:16050 Zbl 1101.76415

[Fomin et al. 2008] S. Fomin, M. Shapiro, and D. Thurston, "Cluster algebras and triangulated surfaces, I: Cluster complexes”, Acta Math. 201:1 (2008), 83-146. MR 2448067 Zbl 05508850

[Gabriel 1981] P. Gabriel, "The universal cover of a representation-finite algebra”, pp. 68-105 in Representations of algebras (Puebla, 1980), edited by M. Auslander and E. Lluis, Lecture Notes in Math. 903, Springer, Berlin, 1981. MR 83f:16036 Zbl 0481.16008 
[Happel et al. 1996] D. Happel, I. Reiten, and S. O. Smalø, Tilting in abelian categories and quasitilted algebras, Mem. Amer. Math. Soc. 575, Amer. Math. Soc., Providence, 1996. MR 97j:16009 Zbl 0849.16011

[Hatcher 1991] A. Hatcher, “On triangulations of surfaces”, Topology Appl. 40:2 (1991), 189-194. MR 92f:57020 Zbl 0727.57012

[Huard and Liu 2000] F. Huard and S. Liu, "Tilted string algebras", J. Pure Appl. Algebra 153:2 (2000), 151-164. MR 2001j:16018 Zbl 0962.16009

[Keller 2007] B. Keller, "Triangulated Calabi-Yau categories", in Proceedings of the workshop of the ICRA 12 (Torun 2007), 2007.

[Keller 2009] B. Keller, "Deformed Calabi-Yau completions”, preprint, 2009. arXiv 0908.3499v5

[Keller and Reiten 2007] B. Keller and I. Reiten, "Cluster-tilted algebras are Gorenstein and stably Calabi-Yau”, Adv. Math. 211:1 (2007), 123-151. MR 2008b:18018 Zbl 1128.18007

[Labardini-Fragoso 2009] D. Labardini-Fragoso, "Quivers with potentials associated to triangulated surfaces”, Proc. Lond. Math. Soc. (3) 98:3 (2009), 797-839. MR 2500873 Zbl 05551833

[Massey 1991] W. S. Massey, A basic course in algebraic topology, Graduate Texts in Mathematics 127, Springer, New York, 1991. MR 92c:55001 Zbl 0725.55001

[Pogorzały and Skowroński 1991] Z. Pogorzały and A. Skowroński, "Self-injective biserial standard algebras”, J. Algebra 138:2 (1991), 491-504. MR 92f:16012 Zbl 0808.16019

[Roldán 1983] O. Roldán, Tilted algebras of types $\widetilde{A_{n}}, \widetilde{B_{n}}, \widetilde{C_{n}}, \widetilde{B C_{n}}$, Ph.D. thesis, Carleton University, 1983.

[Schiffler 2008] R. Schiffler, "A geometric model for cluster categories of type $D_{n}$ ", J. Algebraic Combin. 27:1 (2008), 1-21. MR 2008k:16025 Zbl 1165.16008

[Schröer 1999] J. Schröer, "Modules without self-extensions over gentle algebras”, J. Algebra 216:1 (1999), 178-189. MR 2000d:16022 Zbl 0994.16013

[Schröer and Zimmermann 2003] J. Schröer and A. Zimmermann, "Stable endomorphism algebras of modules over special biserial algebras", Math. Z. 244:3 (2003), 515-530. MR 2004i:16024 Zbl 1036.16004

[Skowroński 1990] A. Skowroński, "Algebras of polynomial growth", pp. 535-568 in Topics in algebra, Part 1 (Warsaw, 1988), edited by S. Balcerzyk et al., Banach Center Publ. 26, PWN, Warsaw, 1990. MR 93k:16026 Zbl 0729.16005

Communicated by Andrei Zelevinsky

Received 2009-04-08 Revised 2009-06-24 Accepted 2009-08-06

ibrahim.assem@usherbrooke.ca

Département de Mathématiques, Université de Sherbrooke, Sherbrooke, QC J1K2R1, Canada

thomas.brustle@usherbrooke.ca Département de Mathématiques, Université de Sherbrooke, Sherbrooke, QC J1K2R1, Canada

Department of Mathematics, Bishop's University,

College St., Sherbrooke, QC J1M0C8, Canada

gabrielle.charbonneau-jodoin@usherbrooke.ca

Département de Mathématiques, Université de Sherbrooke, Sherbrooke, QC J1K2R1, Canada

plamondon@math.jussieu.fr

Institut de Mathématiques,

Université Denis Diderot (Paris VII),

Case 7012-2, place Jussieu 75251 Paris Cedex 05, France 


\section{Guidelines for Authors}

Authors may submit manuscripts in PDF format on-line at the Submission page at the ANT website.

Originality. Submission of a manuscript acknowledges that the manuscript is original and and is not, in whole or in part, published or under consideration for publication elsewhere. It is understood also that the manuscript will not be submitted elsewhere while under consideration for publication in this journal.

Language. Articles in ANT are usually in English, but articles written in other languages are welcome.

Required items. A brief abstract of about 150 words or less must be included. It should be self-contained and not make any reference to the bibliography. If the article is not in English, two versions of the abstract must be included, one in the language of the article and one in English. Also required are keywords and subject classifications for the article, and, for each author, postal address, affiliation (if appropriate), and email address.

Format. Authors are encouraged to use $\mathrm{LT}_{\mathrm{E}} \mathrm{X}$ but submissions in other varieties of $\mathrm{T}_{\mathrm{E}} \mathrm{X}$, and exceptionally in other formats, are acceptable. Initial uploads should be in PDF format; after the refereeing process we will ask you to submit all source material.

References. Bibliographical references should be complete, including article titles and page ranges. All references in the bibliography should be cited in the text. The use of $\mathrm{BibT}_{\mathrm{E}} \mathrm{X}$ is preferred but not required. Tags will be converted to the house format, however, for submission you may use the format of your choice. Links will be provided to all literature with known web locations and authors are encouraged to provide their own links in addition to those supplied in the editorial process.

Figures. Figures must be of publication quality. After acceptance, you will need to submit the original source files in vector graphics format for all diagrams in your manuscript: vector EPS or vector PDF files are the most useful.

Most drawing and graphing packages (Mathematica, Adobe Illustrator, Corel Draw, MAT$\mathrm{LAB}$, etc.) allow the user to save files in one of these formats. Make sure that what you are saving is vector graphics and not a bitmap. If you need help, please write to graphics@mathscipub.org with details about how your graphics were generated.

White Space. Forced line breaks or page breaks should not be inserted in the document. There is no point in your trying to optimize line and page breaks in the original manuscript. The manuscript will be reformatted to use the journal's preferred fonts and layout.

Proofs. Page proofs will be made available to authors (or to the designated corresponding author) at a Web site in PDF format. Failure to acknowledge the receipt of proofs or to return corrections within the requested deadline may cause publication to be postponed. 


\section{Algebra \& Number Theory}

Volume $4 \quad$ No. $2 \quad 2010$

Canonical extensions of Néron models of Jacobians BRYDEN CAIS

Period, index and potential III

Pete L. Clark and Shahed Sharif

On the dimension of $H$-strata in quantum algebras

JASON P. BELL and STÉPHANE LAUNOIS

Gentle algebras arising from surface triangulations

IBRAHIM ASSEM, ThOMAS BRÜSTLE, GABRIELLE CHARBONNEAU-JodoIN and PIERRE-GUY PLAMONDON 